\title{
Determination of the Ultimate Capacity of Elastomeric Bearings Under Axial Loading
}

Gregory L. Bradley and Peter C. Chang

University of Maryland

College Park, Maryland

and

Andrew W. Taylor

KPFF Consulting Engineers

Seattle, Washington

Formerly, National Institute of Standards and Technology

Gaithersburg, Maryland

Building and Fire Research Laboratory

Gaithersburg, Maryland 20899

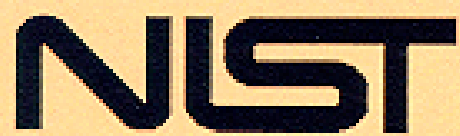

United States Department of Commerce

Technology Administration

National Institute of Standards and Technology 


\section{Determination of the Ultimate Capacity of Elastomeric Bearings Under Axial Loading}

Gregory L. Bradley and Peter C. Chang

University of Maryland

College Park, Maryland

and

Andrew W. Taylor

KPFF Consulting Engineers

Seattle, Washington

Formerly, National Institute of Standards and Technology

Gaithersburg, Maryland

February 1998

A report to:

U.S. Department of Commerce

Technology Administration

National Institute of Standards and Technology

Building and Fire Research Laboratory

Gaithersburg, MD 20899

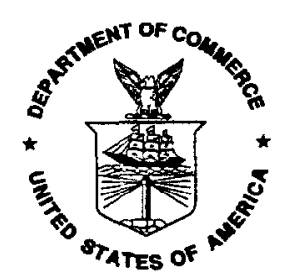

U.S. Department of Commerce

William M. Daley, Secretary

Technology Administration

Gary R. Bachula, Acting Under Secretary for Technology

National Institute of Standards and Technology

Raymond G. Kammer, Director 


\section{BLANK PAGE}




\section{ABSTRACT \\ DETERMINATION OF THE ULTIMATE CAPACITY OF ELASTOMERIC BEARINGS UNDER AXIAL LOADING}

Elastomeric bearings are used as base isolation systems in order to reduce the response of buildings to earthquake ground motions. In order to facilitate the use of this technology, the National Institute of Standards and Technology (NIST) has published guidelines for testing base isolation systems. NIST seeks to improve the current guidelines for determining the capacity of bearings under axial loading. Very few full-scale bearings have been tested to failure because the capacity of a typical bearing is often beyond the loading capacity of existing testing facilities. This research has sought to determine if tests to failure under axial loading on scale models can be used to predict the axial failure load of a full-scale bearing. Tests to failure were conducted on full-scale, 1/2-scale, and 1/4-scale bearings. However, there were dissimilarities in steel shim thickness, cover layer, and the central alignment hole. The stress-strain responses were similar, but not identical. Nonlinear finite element analysis conclusively demonstrates that similarity in shim and rubber thickness is most important. The dissimilarities in the cover layer and central alignment holes, separately considered, had less effect on the bearing response than shim thickness dissimilarities, and offset each other in this particular case. Tests indicate that the mechanical properties of the 22-gage shims ( $0.762 \mathrm{~mm}$ thick) for the 1/4-scale bearing may be slightly modified, possibly due to sandblasting and/or the cold rolling processing of the steel.

Key Words: building technology; compression testing; earthquake engineering; elastomeric bearings; seismic base isolation; ultimate load tests; nonlinear finite elements; Valanis-Landel Function; rubber modeling. 


\section{BLANK PAGE}




\section{PREFACE}

Any mention of commercial items serves only to adequately specify the analysis and experimental procedures. In no case does such identification imply recommendation or endorsement by the National Institute of Standards and Technology, nor does it imply that this product is necessarily the best available for the purpose. 
BLANK PAGE 


\section{ACKNOWLEDGEMENTS}

This research program was supported by the Structures Division, Building and Fire Research Laboratory, National Institute of Standards and Technology, U.S.

Department of Commerce through a grant to the University of Maryland.

This work would not have been possible without the guidance and comments from Dr. Greg McKenna (Polymers Division at NIST) on the mechanics of rubber. The authors would also like to thank Drs. Fahim Sadek and Tim Whalen for their many helpful suggestions and comments. 


\section{BLANK PAGE}




\section{TABLE OF CONTENTS}

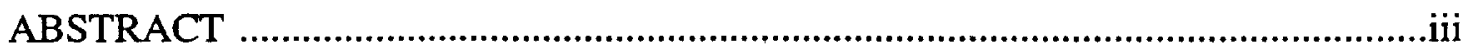

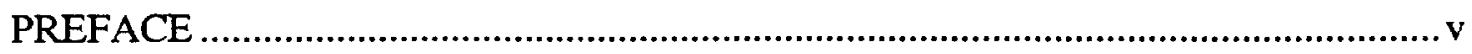

ACKNOWLEDGEMENTS .................................................................................

TABLE OF CONTENTS ..................................................................................

LIST OF FIGURES ........................................................................................

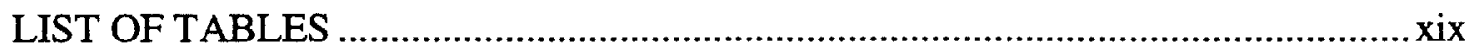

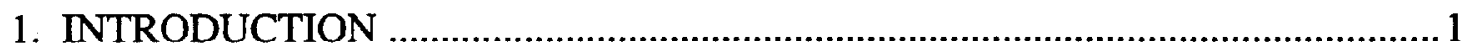

2. UNIAXIAL COMPRESSION TESTING ON BEARINGS …...............................

2.1 Description of Test Specimens …........................................................ 3

2.1.1 Dimensions ........................................................................... 3

2.1.2 Rubber Properties Reported by Bearing Manufacturers ..................... 5

2.1.2.1 Rubber for B-Bearings .........................................................5

2.1.2.2 Rubber for A-Bearing .....................................................6

2.2 Test Machine and Bearing Setup ........................................................... 7

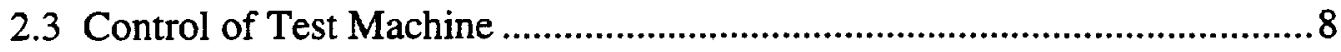

2.4 Test Procedure ............................................................................... 8

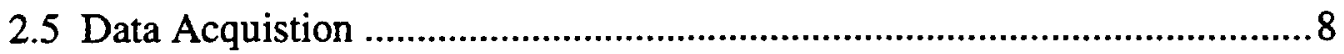

2.6 Observations and Results of $\mathrm{A}$ and B-Bearing Tests.............................9

2.6.1 Bearing A - First Test .................................................................... 9

2.6.2 Bearing A - Second Test ............................................................. 9

2.6.3 Full-Scale Bearing (B1) Test ...................................................... 10

2.6.3.1 Visual Observations and Load vs Displacement Curve for Full-Scale Bearing (B1) Test ................................................ 10

2.6.3.2 Head Velocity and Load During Failure for Full-Scale Bearing (B1) Test ................................................................ 10

2.6 .4 1/2-Scale Bearing (B2) Test ............................................................ 11

2.6.4.1 Visual Observations and Load vs Displacement Curve for $1 / 2$-Scale Bearing (B2) Test ............................................... 11

2.6.4.2 Head Velocity and Load During Failure for $1 / 2-$ Scale Bearing (B2) Test ................................................................... 11

2.6 .5 1/4-Scale Bearing (B3) Test ......................................................... 11

2.6.5.1 Visual Observations and Load vs Displacement Curve for 1/4-Scale Bearing (B3) Test 
2.6.5.2 Head Velocity and Load During Failure for 1/4-Scale Bearing (B3) Test 12

2.6.6 Comparisons among $A$ and B-Bearing Tests...................................... 12

2.7 Measurement Accuracy and Variability ...................................................13

3. DETERMINATION OF RUBBER STRESS-STRAIN PROPERTIES

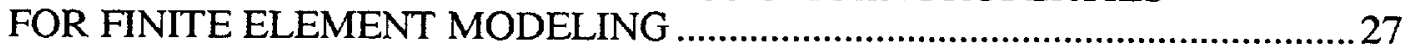

3.1 Background for Finite Element Modeling ……......................................22

3.2 Theoretical Basis for Constitutive Modeling of Rubber.............................28

3.3 Experiments Performed on Rubber for Finite Element

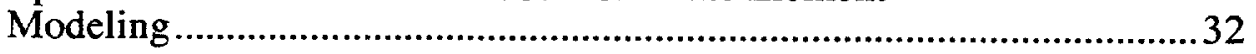

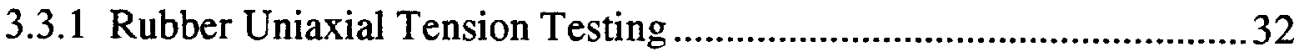

3.3.1.1 Rubber Uniaxial Tension Testing Procedure .................................32

3.3.1.2 Rubber Uniaxial Tension Testing - Observations and

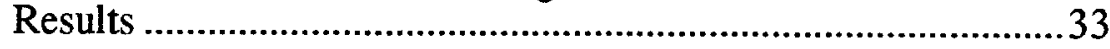

3.3.2 Rubber Uniaxial Compression Testing ...............................................33

3.3.2.1 Rubber Uniaxial Compression Testing Procedure ........................33

3.3.2.2 Rubber Uniaxial Compression Testing - Observations

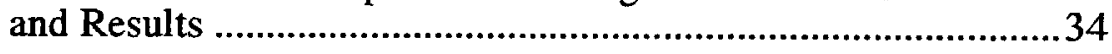

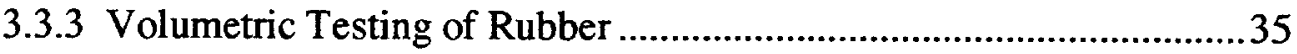

3.3.4 Rubber Model used in Finite Element Analysis (based on

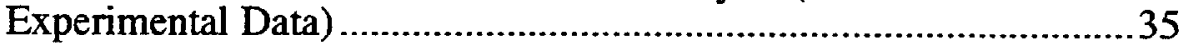

3.3.5 Measurement Accuracy and Variability and Effect on

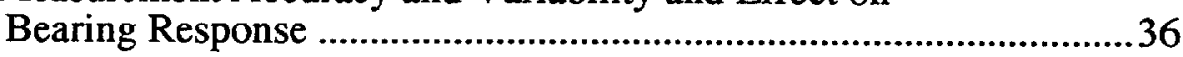

3.4 Extension of Rubber Experimental Stress-Strain Data to Equibiaxial and Planar Deformation Modes using the Valanis-Landel Function 36

3.5 Assessment of the Valanis-Landel Function ..............................................41

3.6 Rubber Models used in Finite Element Analysis (based on Experimental and Valanis-Landel Generated Data).

4. INVESTIGATION OF THE RUBBER MODELS USED IN THE

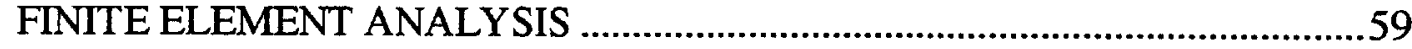

4.1 Calibration of the Rubber Models in ABAQUS ......................................59

4.2 Reduced Stress Plots....................................................................................64

5. FINITE ELEMENT MODELING OF STEEL_......................................................

5.1 Steel Testing Methods ...........................................................................79

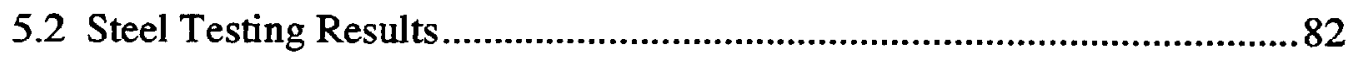


5.3 Measurement Accuracy and Variability and Effect on Bearing Response

\section{FINITE ELEMENT MODELING OF THE ELASTOMERIC}

BEARINGS

6.1 Discretization of the Finite Element Models of the Bearings

6.2 Effect of Different Factors on the Results of the Finite Element Model

6.2.1 Inclusion of the Central Core in the Model 89

6.2.2 Comparison between the Ogden and Polynomial Strain Energy Functions and Rubber Models RM1 and VL1 .....................90

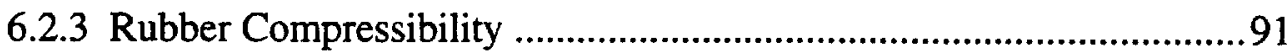

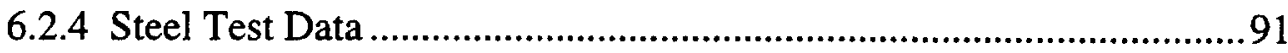

6.3 Deformation and Contour Plots and the Dominance of Hydrostatic Stress

6.4 Discussion of Differences in Initial Stiffness of Finite Element Model versus Experiment

6.5 Results of Finite Element Analysis on Scale Models and Geometric Dissimilarities

7. CONCLUSIONS 123

REFERENCES 127

APPENDIX A - SOME ELEMENTARY RESULTS IN LARGE STRAIN KINEMATICS

APPENDIX B - PROGRAM LISTING FOR DETERMINATION OF THE DERIVATIVE OF THE VALANIS-LANDEL FUNCTION 


\section{BLANK PAGE}




\section{LIST OF FIGURES}

Figure 2.1 - Bearing A dimensions ................................................................ 14

Figure 2.2 - Full-Scale Bearing (B1) dimensions ................................................ 15

Figure 2.3 - Bearing dimensions for 1/2-Scale (B2) and 1/4-Scale (B3) Bearings ......16

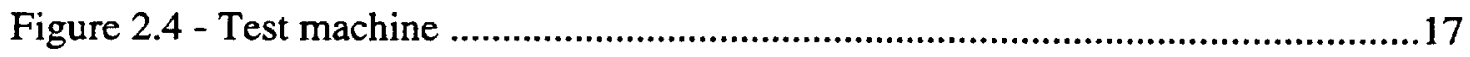

Figure 2.5 - Setup for Bearing A tests ............................................................ 18

Figure 2.6 - Setup for geometrically similar bearings (B) tests ...............................19

Figure 2.7 - Load-head speed vs load for Bearing A, initial test (2nd cycle) ...............20

Figure 2.8 - Load vs displacement plot for Bearing A, first test ................................20

Figure 2.9 - Load vs displacement plot for Bearing A, second test ..........................21

Figure 2.10 - Load vs displacement plot for Full-Scale Bearing (B 1) ........................21

Figure 2.11 - Head velocity and load during shim failures for Full-Scale Bearing (B1) ...........................................................................22

Figure 2.12 - Load vs displacement plot for 1/2-ScaleBearing (B2) ..........................22

Figure 2.13 - Head velocity and load during shim failures for $1 / 2-S c a l e$

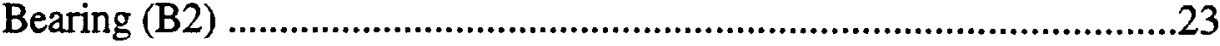

Figure 2.14 - Load vs displacement plot for 1/4-Scale Bearing (B3) ........................23

Figure 2.15 - Head velocity and load during shim failures for $1 / 4-$ Scale Bearing (B3) ...........................................................................24

Figure 2.16 - Comparison of stress-strain curves for all bearings ............................24

Figure 2.17 - Comparison of adjusted stress-strain curves for B-Bearings .................25

Figure 3.1 - Effect of mechanical conditioning on the stress-strain response of rubber .43

Figure 3.2 - Rubber sheets from which tensile specimens were cut ..........................43

Figure 3.3 - Dimensions of rubber tensile specimens ...........................................44

Figure 3.4 - Stress-strain curves for all rubber tensile tests ......................................44

Figure 3.5 - Dimensions of rubber specimens used for compression testing …...........45

Figure 3.6 - Homogeneous vs bonded compression ..............................................46 
Figure 3.7 - Comparison of compressive response of two compression set specimens

Figure 3.8 - Comparison of compressive response of compression set and durometer specimens

Figure 3.9 - Device used for volumetric testing …..................................................48

Figure 3.10 - Comparison of volumetric compression tests .........................................49

Figure 3.11 - CompSet 7 (CS7) volumetric compression test .....................................49

Figure 3.12 - Rubber model 1 (RM1) ………………….........................................50

Figure 3.13 - Volumetric compression plot (based on CS7) .......................................50

Figure 3.14 - True stress-strain data set used to find the derivative of the Valanis-Landel Function $u^{\prime}(\lambda)$

Figure 3.15 - Polynomial curvefit used to obtain a functional representation of the stress-strain curve for determination of the derivative of the Valanis-Landel Function $u^{\prime}(\lambda)$ for $0.14<1<0.3$

Figure 3.16 - Derivative of the Valanis-Landel Function $u^{\prime}(\lambda)$

Figure 3.17 - Comparison between the experimental uniaxial tension test and the uniaxial tension derived from the Valanis-Landel function

Figure 3.18 - Comparison between the experimental uniaxial compression test and the uniaxial compression derived from the Valanis-Landel function

Figure 3.19 - Equivalence of uniaxial compression and equibiaxial tension for an incompressible material

Figure 3.20 - Comparison between the equibiaxial tension converted from uniaxial compression and the equibiaxial tension derived from the Valanis-Landel function

Figure 3.21 - Comparison between the experimental uniaxial tension test and the planar tension derived from the Valanis-Landel function

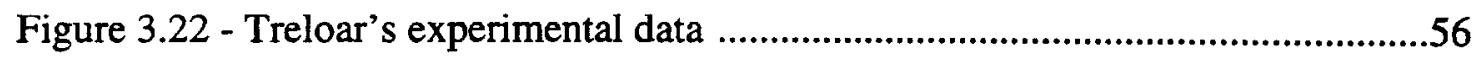

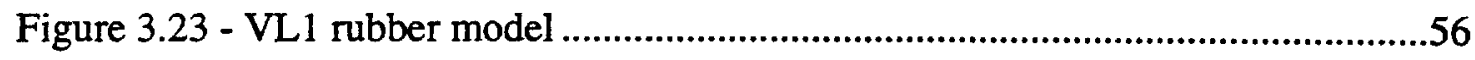

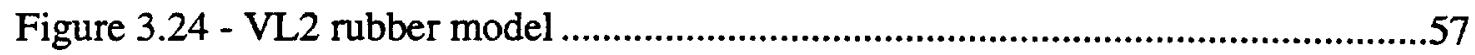

Figure 4.1 - Plot of reduced stresses for the experimental uniaxial tensile and compressive stress-strain data 
Figure 4.2 - Calibration to uniaxial tension with compressibility using RM1 .67

Figure 4.3 - Calibration to uniaxial tension without compressibility using RM1 ........67

Figure 4.4 - Calibration to uniaxial tension with compressibility using VL1 ..............68

Figure 4.5 - Calibration to uniaxial tension with compressibility using VL2 ..............68

Figure 4.6 - Calibration to uniaxial compression with compressibility using RM1

Figure 4.7 - Calibration to uniaxial compression without compressibility using RM1

Figure 4.8 - Calibration to uniaxial compression with compressibility using VL1 ......70

Figure 4.9 - Calibration to uniaxial compression with compressibility using VL2 ......70

Figure 4.10 - Calibration to equibiaxial tension with compressibility using RM1 ......71

Figure 4.11 - Calibration to equibiaxial tension without compressibility using RM1

Figure 4.12 - Calibration to equibiaxial tension with compressibility using VL1 .......72

Figure 4.13 - Calibration to equibiaxial tension with compressibility using VL2 _......72

Figure 4.14 - Calibration to planar tension with compressibility using RM1 ..............73

Figure 4.15 - Calibration to planar tension without compressibility using RM1 .........73

Figure 4.16 - Calibration to planar tension with compressibility using VL1 ...............74

Figure 4.17 - Calibration to planar tension with compressibility using VL2 ................74

Figure 4.18 - Calibration to volumetric compression test using RM1 and VL1 models

Figure 4.19 - Reduced uniaxial stresses from experiment vs ABAQUS results, using RM1

Figure 4.20 - Reduced uniaxial stresses from experiment vs ABAQUS results, using VLl

Figure 4.21 - Reduced uniaxial stresses from experiment vs. ABAQUS results, using VL2

Figure 5.1 - Dimensions of tensile dogbone specimens

Figure 5.2 - Micrometers used to measure the necking region of the tensile dogbone specimens. 
Figure 5.3 - True stress-strain curves for smooth (S) 12-gage specimens...................85

Figure 5.4 - True stress-strain curves for sandblasted (SB) 12-gage specimens ..........85

Figure 5.5 - True stress-strain curves for chemlocked (CL) 12-gage specimens .........86

Figure 5.6 - True stress-strain curves for sandblasted (SB) 16-gage specimens ..........86

Figure 5.7 - True stress-strain Curves for sandblasted (SB) 22-gage specimens by finish

Figure 5.8 - Comparison of true stress-strain curves for 12-gage steel samples ..........87

Figure 5.9 - Comparison of 12,16 , and 22-gage steel samples .............................88

Figure 5.10 - Comparison of 12,16, and 22-gage steel samples up to a

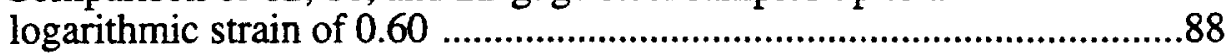

Figure 6.1 - Undeformed mesh for Full-Scale Bearing .........................................96

Figure 6.2 - Undeformed mesh for $1 / 2$-Scale Bearing ..........................................97

Figure 6.3 - Undeformed mesh for 1/4-Scale Bearing ..........................................98

Figure 6.4 - Photograph of central slice of failed 1/2-Scale Bearing (B2) ...................99

Figure 6.5 - Deformed shape and steel plastic strains in the prototype modeled with the central rubber core

Figure 6.6 - Comparison for the prototype between the experimental engineering stress strain curve and $\mathrm{ABAQUS}$ finite element runs with and without the modeling of the core

Figure 6.7 - Comparison of ABAQUS runs based on Ogden, $\mathrm{N}=6$ and Polynomial, $N=2$ Strain Energy Functions.

Figure 6.8 - Comparison for the prototype between the experimental engineering stress strain curve and the ABAQUS finite element runs based on RM1 and VL1

Figure 6.9 - Comparison for the prototype between the experimental engineering stress-strain curve and the ABAQUS finite element runs using a compressible versus an incompressible rubber model 102

Figure 6.10 - Low strain region comparison for the prototype between the experimental engineering stress-strain curve and the ABAQUS finite element runs using a compressible versus an incompressible rubber model

Figure 6.11 - Comparison for the prototype between the experimental engineering stress-strain curve and the ABAQUS finite element 
runs using the chemlocked steel data (CL1-12) versus the sandblasted steel data (SB2-12)

Figure 6.12 - Comparison between the true stress-strain curves of the chemlocked steel data (CL1-12) versus the sandblasted steel data (SB2-12)

Figure 6.13 - Full-Scale Bearing - Deformation and hydrostatic stresses in the rubber at the compressive engineering failure strain

Figure 6.14 - Full-Scale Bearing - Deformation and major principal stresses in the rubber at the compressive engineering failure strain

Figure 6.15 - Full-Scale Bearing - Deformation and intermediate principal stresses in the rubber at the compressive engineering failure strain

Figure 6.16 - Full-Scale Bearing - Deformation and minor principal stresses in the rubber at the compressive engineering failure strain

Figure 6.17 - Full-Scale Bearing - Deformation and maximum shearing stresses in the rubber at the compressive engineering failure strain

Figure 6.18 - Full-Scale Bearing - Deformation and maximum shearing stresses (up to $7.30 \mathrm{MPa}$ ) in the rubber at the compressive engineering failure strain

Figure 6.19 - Full-Scale Bearing - Deformation and hydrostatic stresses in the rubber at the compressive engineering strain corresponding to initial shim yielding

Figure 6.20 - Full-Scale Bearing - Deformation and major principal stresses in the rubber at the compressive engineering strain corresponding to initial shim yielding

Figure 6.21 - Full-Scale Bearing - Deformation and intermediate principal stresses in the rubber at the compressive engineering strain corresponding to initial shim yielding

Figure 6.22 - Full-Scale Bearing - Deformation and minor principal stresses in the rubber at the compressive engineering strain corresponding to initial shim yielding

Figure 6.23 - Full-Scale Bearing - Deformation and maximum shearing stresses in the rubber at the compressive engineering strain corresponding to initial shim yielding

Figure 6.24 - Full-Scale Bearing - Deformation and von Mises stresses in the steel at the compressive engineering strain corresponding to initial shim yielding

Figure 6.25 - Full-Scale Bearing - Deformation and von Mises stresses in the steel at the compressive engineering failure strain 
Figure 6.26 - Full-Scale Bearing - Deformation and equivalent plastic strain in the steel at the compressive failure engineering strain.

Figure 6.27 - Low strain region comparison for the prototype between the experimental engineering stress-strain curve and the ABAQUS finite element runs based on RM1 and VL

Figure 6.28 - Comparison for the 1/2-Scale Bearing model between the experimental engineering stress-strain curve and the ABAQUS finite element runs using the chemlocked steel data (CL1-12) versus the sandblasted steel data (SB1-16)

Figure 6.29 - Comparison between the true stress-strain curves of the chemlocked steel data (CL1-12) versus the sandblasted steel data (SB1-16)

Figure 6.30 - Comparison for the 1/4-Scale Bearing model between the experimental engineering stress-strain curve and the ABAQUS finite element runs using the chemlocked steel data (CL1-12) versus the sandblasted steel data (SB2-22)

Figure 6.31 - Comparison between the true stress-strain curves of the chemlocked steel data (CL1-12) versus the sandblasted steel data (SB2-22)

Figure 6.32 - Comparison between the ABAQUS finite element runs for the prototype, $1 / 2$ and $1 / 4$ scale models using geometrically nonsimilar and similar shims for the prototype

Figure 6.33 - Separate and combined effects of core and cover layer dissimilarities on the response of the 1/4-Scale Bearing

Figure A.1 - Mapping from the Undeformed to Deformed Configuration 130 


\section{LIST OF TABLES}

Table 2.1(a) - Bearing dimensions .........................................................................

Table 2.2 - B-Bearings - rubber batches ...........................................................6

Table 2.3 - ASTM test results for elastomers .......................................................6

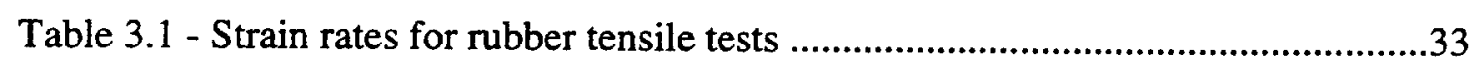

Table 3.2 - Calculated values of the derivative of the

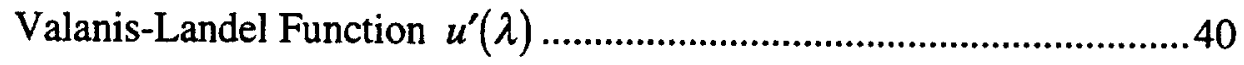

Table 4.1 - Rubber calibrations ........................................................................61

Table 4.2 - Test data included in each rubber model ...............................................62

Table 4.3 - Average RMS error of calibration runs for uniaxial tension .....................62

Table 4.4 - Average RMS error of calibration runs for uniaxial compression .............63

Table 4.5 - Average RMS error of calibration runs for equibiaxial tension .................63

Table 4.6 - Average RMS error of calibration runs for planar tension .......................64

Table 5.1 - Tensile dogbone specimens by finish ................................................80

Table 5.2 - Comparison of elastic properties for steel specimens ...............................83

Table 6.1 - Summary of geometric dissimilarities....................................................95 


\section{BLANK PAGE}




\section{INTRODUCTION}

Seismic base isolation is one of the most promising concepts in seismic resistant construction to come of age in this century. Most of the energy of an earthquake occurs at a period very near the fundamental period of low to mid-rise buildings. Base isolation reduces the response of the structures of this size to earthquake ground motions by shifting the structure's fundamental period away from the dominant period of the ground shaking. In an effort to facilitate the use of this technology, the National Institute of Standards and Technology (NIST) has published Guidelines for Pre-Qualification, Prototype and Quality Control Testing of Seismic Isolation Systems (NISTIR 5800, 1996).

Base isolation systems provide the lateral flexibility and damping necessary for effective isolation, and the high initial stiffness required to resist wind loads. Isolation systems are broadly grouped into three categories: elastomeric, sliding, and hybrid. A common type of elastomeric isolation system is the ordinary laminated bearing, which consists of layers of elastomer and steel bonded under high temperature and pressure to form an integral bearing that is free of joints (elastomer and rubber are used interchangeably throughout this report). The restoring force and damping mechanism are combined in a single device. The isolation units studied in this research are of the laminated bearing type. Sliding systems rely on simple Coulomb friction between two surfaces, and hybrid systems can incorporate aspects of both elastomeric and sliding types, but generally use independent components to provide the restoring force, damping, wind restraint and ultimate restraint.

Very few full-scale isolation units have been tested to ultimate failure because the bearing capacity of a typical bearing is often beyond the loading capacity of existing testing facilities. As a result, the factor of safety of these systems is unknown (NISTIR 5800, Section 8.2,1996). A simple way around this problem is to test scalemodel versions of the bearings. If the material and geometric properties are properly scaled, then similitude relationships can be used to predict the failure load of a fullscale bearing based on tests of a scale model bearing. However, a perfectly geometrically similar scaled model is difficult to make because some dimensions are predetermined by the industry, e.g. standard bearing molds are frequently not geometrically similar, resulting in dissimilar diameters and central cores. Variation of material through the thickness, if any, may be impossible to control. The major goals of this study are to establish the geometric properties that must be similar in order to build scale models that will fail at the same stress and strain as a full-scale bearing, and to determine if the material properties of the steel shims change significantly in scale models. Four laminated bearings, three of which are nearly geometrically similar, were tested. Finite element analyses were performed to investigate the effect of geometric dissimilarities on the prediction of ultimate load from scale model tests. 


\section{BLANK PAGE}




\section{UNIAXIAL COMPRESSION TESTING ON BEARINGS}

\subsection{Description of Test Specimens}

\subsubsection{Dimensions}

Four specimens were used in the study. The specimens are designated as $\mathrm{A}$ and B1 through B3. Specimen B1 is a Full-Scale (or prototype) elastomeric bearing. Specimens B2 and B3 are respectively $1 / 2$-Scale and $1 / 4$-Scale versions of Specimen B1. These specimens were produced by the same manufacturer with the goal of minimizing any differences in material properties between specimens. Specimen $A$ is not geometrically similar to the B-Bearings, and was manufactured by a different company. All specimens were fabricated using materials and fabrication procedures typical of those used in the manufacture of commercial isolators. Table 2.1 lists the important dimensions of each bearing, and Figures 2.1 through 2.3.(b) are dimensioned drawings of Bearings A, B1, B2, and B3, respectively.

The Full-Scale Bearing (B1) was not designed for a specific application. It was sized to have a slightly lower capacity than a typical full-scale isolator in order to ensure failure in the testing facilities available at NIST.

Even though strict geometric similarity was desired, the as-built B-Bearings were not geometrically similar. The dissimilarities follow.

(1) Layer Thicknesses - For geometric similarity to hold, each shim and rubber layer in the Full-Scale Bearing must be twice as thick as the 1/2-Scale Bearing and four times as thick as 1/4-Scale Bearing. Due to a manufacturing error the shims for the Full-Scale Bearing are less than twice the thickness of the shims for the 1/2Scale Bearing. Geometric similarity in shim thickness is maintained in the $1 / 2$-Scale and 1/4-Scale Bearings, and in rubber thickness for all B-Bearings.

(2) Bearing Diameters - The outer diameters of the B-Bearings could not be made geometrically similar because the manufacturer used readily available molds. The outer diameters of the bonded areas, which correspond to the shim diameters, are geometrically similar. Therefore, the cover layers, which protect the shims from environmental exposure, are not geometrically similar.

(3) Center Alignment Hole - The center alignment holes are used to facilitate alignment of the bearing components during manufacture, and are filled with rubber after stacking of the shim and rubber layers. They are not geometrically similar. 
Table 2.1(a) - Bearing dimensions

\begin{tabular}{lcccc}
\hline & A & $\begin{array}{c}\text { Full-Scale } \\
\text { B1 }(\mathrm{mm})\end{array}$ & $\begin{array}{c}1 / 2-\text { Scale } \\
\text { B2 }(\mathrm{mm})\end{array}$ & $\begin{array}{c}\text { 1/4-Scale } \\
\text { B3 }(\mathrm{mm})\end{array}$ \\
\hline Total Height & 584 & 304 & 167 & 109 \\
Total Rubber Layer Thickness & 392 & $191^{*}$ & $95^{*}$ & $48^{*}$ \\
Total Shim Thickness & 115.6 & 37.2 & $21.3^{*}$ & $10.7^{*}$ \\
Thickness: Each End Plate & 38.1 & 38.1 & 25.4 & 25.4 \\
\hline
\end{tabular}

Table 2.1(b) - Bearing dimensions

\begin{tabular}{rcccc}
\hline & A & $\begin{array}{c}\text { Full-Scale } \\
\text { B1 }\end{array}$ & $\begin{array}{c}1 / 2-\text { Scale } \\
\text { B2 }\end{array}$ & $\begin{array}{c}1 / 4-\text { Scale } \\
\text { B3 }\end{array}$ \\
\hline Rubber Layers & & & & \\
\# of Layers & 39 & 15 & 15 & 15 \\
Thickness (mm) & 10.1 & $12.7^{*}$ & $6.4^{*}$ & $3.2^{*}$ \\
Shape Factor & 24.4 & $15^{*}$ & $15^{*}$ & $15^{*}$ \\
Total Diameter (mm) & 1016 & 800 & 406 & 229 \\
Bonded Dia & 991 & $762^{*}$ & $381^{*}$ & $191^{*}$ \\
(mm) & $2 \times 12.5$ & $2 \times 19$ & $2 \times 12.5$ & $2 \times 19$ \\
Cover Layer & & & & \\
Thickness (mm) & & & & \\
\hline & & & &
\end{tabular}

${ }^{*}$ Geometrically similar dimension.

${ }^{* *}$ Shape factor (S) is defined as the bonded plan area of the bearing (one side only) divided by the area of the edge of a single layer of elastomer $(S=d / 4 t)$, where $d$ is the bonded diameter and $t$ is the rubber layer thickness). 
Table 2.1(b) (continued) - Bearing dimensions

\begin{tabular}{|c|c|c|c|c|}
\hline & A & $\begin{array}{c}\text { Full-Scale } \\
\text { B1 }\end{array}$ & $\begin{array}{c}\text { 1/2-Scale } \\
\text { B2 }\end{array}$ & $\begin{array}{c}\text { 1/4-Scale } \\
\text { B3 }\end{array}$ \\
\hline \multicolumn{5}{|l|}{ Steel Shims } \\
\hline No. of shims & 38 & 14 & 14 & 14 \\
\hline Thickness (gage) & 11 gage $^{*}$ & 12 gage & 16 gage $^{*}$ & 22 gage * \\
\hline$(\mathrm{mm})$ & $3.048^{*}$ & 2.667 & $1.524^{*}$ & $0.762^{*}$ \\
\hline Diameter (mm) & 991 & $762^{*}$ & $381^{*}$ & $191^{*}$ \\
\hline $\begin{array}{l}\text { Center Alignment } \\
\text { Hole Diameter }{ }^{\dagger}(\mathrm{mm})\end{array}$ & 76.2 & 50.1 & 30.1 & 30.1 \\
\hline
\end{tabular}

End Plate

$\begin{array}{lcccc}\text { Plan Dimensions } & 991 \mathrm{~mm} \\ (\mathrm{~mm}) & \text { diameter } & \begin{array}{c}876 \mathrm{~mm} \text { edge } \\ \text { length }\end{array} & \begin{array}{c}508 \mathrm{~mm} \text { edge } \\ \text { length }\end{array} & \begin{array}{c}305 \mathrm{~mm} \text { edge } \\ \text { length }\end{array}\end{array}$

* Geometrically similar dimension.

$\dagger$ Holes are placed in shim plates to facilitate alignment of the bearing components during manufacture.

\subsubsection{Rubber Properties Reported by Bearing Manufacturers}

The following sections present the results of ASTM tests on the rubber compounds, as reported by the bearing manufacturers. Both bearing types use a highdamping natural rubber vulcanizate.

\subsubsection{Rubber for B-Bearings}

All natural rubber used for these bearings came from the same shipment. The natural rubber is mixed with various chemicals, but due to the size of the rubber mixing equipment, three batches were required. Four batches were mixed, but plant quality control rejected the first batch (the rejected batch had been calendered (rolled) to the wrong thickness). Each batch was made from the same proportions of identical ingredients. The rubber was vulcanized only after placing the shims and the rubber in alternating layers into a mold.

Two bearings of each size were manufactured; one bearing of each size was tested in this program. The second bearing will be tested to failure in compression with an applied lateral load in a later test program. Table 2.2 shows the batch numbers for each bearing. 
Table 2.2 - B-Bearings - rubber batches

\begin{tabular}{ccc}
\hline Bearing & $\begin{array}{c}\text { Ultimate Compression } \\
\text { No Lateral Load }\end{array}$ & $\begin{array}{c}\text { Ultimate Compression Lateral } \\
\text { Load Applied } \\
\text { (Future Test Program) }\end{array}$ \\
\hline B1 & Batch 3 & Batch 2 \\
B2 & Batch 3 & Batch 3 \\
B3 & Batch 4 & Batch 4 \\
\hline
\end{tabular}

As noted above, Batch 1 was rejected by plant quality control, not because its properties differed from the other three batches, but because it was calendered to the wrong thickness. For ASTM testing, four of the six tests were conducted using specimens made from a composite batch, which consisted of equal quantities from each of the four batches. The results of ASTM testing are shown in Table 2.3.

\subsubsection{Rubber for A-Bearing}

Test results of the rubber used in the particular bearing tested were not available. The manufacturer, however, reported test results for elastomers with the same chemical composition. These results are shown in Table 2.3.

Table 2.3 - ASTM test results for elastomers

\begin{tabular}{rcccc}
\hline & $\begin{array}{c}\text { Shear Modulus } \\
\text { (ASTM D } \\
\text { 4014-89, } \\
\text { Annex A1) } \\
(\mathrm{psi})\end{array}$ & $\begin{array}{c}\text { Tensile } \\
\text { Strength } \\
\text { (ASTM D 412- } \\
\text { 92, Method A) } \\
(\mathrm{psi})\end{array}$ & $\begin{array}{c}\text { Ultimate } \\
\text { Elongation } \\
\text { (ASTM D 412- }\end{array}$ & $\begin{array}{c}\text { Compression } \\
\text { Set }\end{array}$ \\
$\begin{array}{r}\text { (ASTM D 395- } \\
(\%)\end{array}$ & $\begin{array}{c}\text { 89, Method B) } \\
(\%)\end{array}$ \\
\hline $\begin{array}{r}\text { B-Bearings } \\
\text { Composite } \\
\text { Batch }\end{array}$ & 128 & 2406 & 621 & \\
Batch 1 & N/A & 2425 & 597 & 20.75 \\
Batch 2 & N/A & 2537 & 608 & N/A \\
Batch 3 & N/A & 2491 & 613 & N/A \\
Batch 4 & N/A & 2589 & 664 & N/A \\
\hline Bearing A & 173 & $2827-2891$ & $529-579$ & N/A \\
\hline elastomer & & & &
\end{tabular}

N/A denotes that tests were not performed. 
Table 2.3 (continued) - ASTM test results for elastomers

\begin{tabular}{rccc}
\hline & $\begin{array}{c}\text { Shore A Hardness } \\
\text { (ASTM D 2240-91) }\end{array}$ & $\begin{array}{c}\text { Peel Strength } \\
\text { (ASTM D 429-81, } \\
\text { Method B) }\end{array}$ & $\begin{array}{c}\text { Ozone Resistance } \\
\text { (ASTM D 1149-91) }\end{array}$ \\
\hline B-Bearings & & & \\
$\begin{array}{r}\text { Composite } \\
\text { Batch }\end{array}$ & 65 & $\begin{array}{c}\text { 89 lb/in. } \\
100 \% \text { rubber tear }\end{array}$ & $\begin{array}{c}\text { No cracks at } \\
\text { magnification 7X }\end{array}$ \\
Batch 1 & 64 & N/A & N/A \\
Batch 2 & 65 & N/A & N/A \\
Batch 3 & 65 & N/A & N/A \\
Batch 4 & 65 & N/A & N/A \\
\hline $\begin{array}{r}\text { Bearing A } \\
\text { elastomer }\end{array}$ & $63-65$ & $81-93$ lb/in & N/A \\
\hline
\end{tabular}

* The ozone concentration was 50 parts per hundred million (50pphm). The specimens were stretched to $20 \%$ strain, and placed for 100 hours at a temperature of $100^{\circ} \mathrm{F}$.

\subsection{Test Machine and Bearing Setup}

The Universal Testing Machine is located in the Large Scale Structural Testing Facility (Building 202) at the National Institute of Standards and Technology. It is managed by the Building and Fire Research Laboratory. The test machine has a maximum compression capacity of $53.4 \mathrm{MN}$ (12 million pounds). See Figure 2.4. The machine has four large screw columns that rise to approximately $23.8 \mathrm{~m}$ above the ground. The load-head is connected to the four columns, and is positioned by moving it up or down on the screw columns. Once in position, the head is locked in place so that the head does not move relative to the screw columns throughout the test. The screw columns extend to about $7.0 \mathrm{~m}$ below ground, where a hydraulic cylinder assembly is located. The cylinder is approximately $2.1 \mathrm{~m}$ in diameter and is rigidly connected to the four screw columns. As fluid is pumped into the cylinder, the cylinder is pulled down, which in turn pulls the four columns down simultaneously. Consequently, the load-head moves down, pushing the head against the specimen. The maximum stroke of the machine is $1.5 \mathrm{~m}$.

For the A-Bearing tests, the bearing was placed on two large cylindrical steel plates. These were in turm placed on a reinforced concrete platform designed to withstand greater than $53.4 \mathrm{MN}$. See Figure 2.5. For the B-Bearing tests, the reinforced concrete platform was not used. Instead, a third cylindrical piece of steel was placed on top of the two steel plates used in the A-Bearing tests. See Figure 2.6. Also, for each test a smooth steel plate, $6.35 \mathrm{~mm}$ (1/4 in) thick, was placed directly above and below the bearing to ensure a smooth contact surface and to protect the test 
machine load-head from damage that could result from the irregular surface on the top of the bearings.

\subsection{Control of Test Machine}

The test machine is controlled by an open-loop control system. The amount of hydraulic fluid in the hydraulic cylinder assembly determines the head position, and the rate of flow of hydraulic fluid determines the head speed. To control the head speed, the operator manually adjusts the rate of flow of hydraulic fluid. The rate of loading is indicated by the speed of the load indicator and tells the operator if he is allowing too much or too little fluid to move the head. To maintain a constant head velocity (i.e., a constant rate of flow of hydraulic fluid), the operator must continually increase the valve opening to compensate for a reduced flow rate as the fluid pressure rises. However, even with these manual adjustments, head speed decreases as the load approaches the machine's capacity of $53.4 \mathrm{MN}$ (12 million pounds).

\subsection{Test Procedure}

As described in NISTIR 5800, Section 5.4.1, each bearing was subjected to simple axial compression under zero lateral load. Two tests were conducted on Bearing $\mathrm{A}$ in order to gain experience with test machine control and data acquisition prior to conducting the tests on the geometrically similar bearings $\mathrm{B} 1, \mathrm{~B} 2$, and $\mathrm{B} 3$.

It is the usual practice in testing elastomeric bearings to mechanically condition the elastomer, since the initial response of an elastomeric specimen is stiffer than the response after even one cycle of loading. The response of the elastomer typically stabilizes after a number of cycles; however, there is no firm guidance on the number of conditioning cycles needed to attain equilibrium. Researchers of bridge elastomeric bearings have used four (4) mechanical conditioning cycles (Minor and Egen, 1970). For dynamic testing, at least six cycles have been recommended by Brown (1986). In this study, it was decided to use at least six conditioning cycles. In the first test on Bearing $\mathrm{A}$, seven conditioning cycles to $13.79 \mathrm{MPa}(2000 \mathrm{psi})$ compressive stress were applied. In the second test on the A-Bearing and the subsequent tests on the B-Bearings, six conditioning cycles were applied to $13.79 \mathrm{MPa}$ (2000 psi) compressive stress. Six conditioning cycles were adequate to reach an equilibrium stress-strain curve.

\subsection{Data Acquistion}

Four Linear Variable Displacement Transducers (LVDTs) were placed evenly around each bearing in order to measure vertical displacement. These figures were averaged to arrive at the vertical displacement. The LVDTs were not attached to the bearings, but contacted the load head directly, as shown in Figure 2.6. Therefore, any misalignment that might exist between the end plates of the bearings is not measurable. Displacement readings were electronically recorded every $1 / 10$ th of a second. 


\subsection{Observations and Results of A and B-Bearing Tests}

\subsubsection{Bearing A - First Test}

The initial test on the A-Bearing consisted of seven mechanical conditioning cycles followed by five load cycles to the machine capacity of $53.4 \mathrm{MN}$. The test was conducted at the maximum head speed, which was measured at $0.40 \mathrm{~mm} / \mathrm{sec}$.

However, Figure 2.7, which shows a plot of head speed versus load for a typical load cycle, clearly shows that maintaining a constant head velocity was not possible. The average speed over this cycle was $0.30 \mathrm{~mm} / \mathrm{sec}$, with a standard deviation of 0.09 $\mathrm{mm} / \mathrm{sec}$.

During the mechanical conditioning cycles to $13.79 \mathrm{MPa}(2000 \mathrm{psi})$, no bulging of the sides of the bearing was observed. During the load cycles to the machine maximum of $53.4 \mathrm{MN}$, slight bulging was visible. The middle third of the bearing, as viewed from the West, skewed slightly to the North. There was a slight residual bulge and skew at the end of the test.

The load vs displacement plot (Figure 2.8) shows a change in stiffness at approximately $50 \mathrm{MN}$ compressive force $(65 \mathrm{MPa})$ and a compressive strain of 0.042 $\mathrm{m} / \mathrm{m}$ in the first loading cycle. After removal of load, the bearing did not return to its pre-loaded height, thus confirming that inelastic deformation took place during the first cycle. It is reasonable to conclude that the change in slope in the first loading cycle was due to yielding of the steel shims.

Prior to initial yielding, the curve is nearly linear, with a tangent stiffness of $1700 \mathrm{MPa}$. After the initial yielding, the tangent stiffness dropped by $47 \%$ to 900 $\mathrm{MPa}$. The curve shows a concave-upward shape for the second through fifth load cycles. The stress-strain response of pure rubber has a concave-upward shape at higher compressive stresses, and this supports the conclusion that the steel yielded in the initial load cycle to $53.4 \mathrm{MN}$ - the bearing's behavior is dominated more by the rubber than the steel in the second through fifth cycles.

During the unloading portion of the first cycle, the operator inadvertently reversed the flow of hydraulic fluid, causing a slight increase in load for a moment. This is noticeable in the plot.

\subsubsection{Bearing A - Second Test}

Rubber's stress-strain behavior is a function of strain rate (Treloar, 1975). The second test on the A-Bearing was conducted primarily to investigate the influence of strain rate on bearing response. Four sets of three cycles of loading were applied, each to the machine load capacity. The first set was conducted at the maximum head speed, and the second, third and fourth sets at $50 \%, 25 \%$, and $10 \%$ of the maximum head speed, respectively (the maximum head speed measured in these tests was 0.40 $\mathrm{mm} / \mathrm{s}$ ). As shown in Figure 2.9, the stress-strain curves for each set are virtually identical.

The second test was performed seven days after the first. The slight bulge and skew of the bearing in the northerly direction resulting from the first test was still visible prior to the second test. As in the first test, the bulging and lateral deformation increased when the bearing was loaded. 
The general shape of the curves (Figure 2.9) is similar to the curves obtained in the first test after yielding. The vertical compressive strain in the first load cycle of the second test was approximately $0.04 \mathrm{~m} / \mathrm{m}$ at $53.4 \mathrm{MN}$, which is about the same as the vertical strain in the second load cycle of the first test (the first cycle after yielding), with the permanent set due to yielding removed. The plot also shows a slight increase in the maximum displacement in each succeeding load cycle, as in the first test.

The plot also indicates that the bearing response was virtually identical at head speeds ranging from the maximum head speed down to approximately $10 \%$ of the head speed. Therefore, for the limited range of loading rates examined in this study, bearing response to compressive loading can be considered independent of loading rate.

\subsubsection{Full-Scale Bearing (B1) Test}

For each of the B-Bearings tests, the bearings failed as a result of shim rupture. Accordingly, the loading history consisted of six mechanical conditioning cycles followed by loading to failure. In all cases, load was applied well beyond initial shim failure.

\subsubsection{Visual Observations and Load vs Displacement Curve for Full-Scale Bearing (B1) Test}

Bulging was first noticeable at approximately $65 \mathrm{MPa}$ compressive stress and $12 \%$ compressive strain, and increased sharply between 80 and $90 \mathrm{MPa}$. Bulging was greatest at the center, and varied in a parabolic shape from the top to the bottom of the bearing. Bulging appeared to be fairly uniform around the circumference of the bearing. Failure occurred at $90 \mathrm{MPa}$ compressive stress and a compressive strain of $0: 267 \mathrm{~m} / \mathrm{m}$. Failure was indicated by a loss of strength as displacement continued. This phenomenon was accompanied by an audible rupture, followed shortly thereafter by a rapid series of audible ruptures as additional shims failed. Each audible rupture was followed by a sudden drop in compressive load. Loading was stopped when the load had dropped to less than half its peak value.

The stress-strain curve (Figure 2.10) shows initial shim yielding at a compressive stress of about $42 \mathrm{MPa}$ and a compressive strain of about $0.06 \mathrm{~m} / \mathrm{m}$. The shape of the stress-strain curve up to this point is concave-upward, whereas between initial yield and the first shim rupture the shape is concave-downward.

After the initial shim rupture, displacement was increased at the same rate. As shims failed, the load dropped. Small load recovery occurred between shim ruptures.

\subsubsection{Head Velocity and Load During Failure for Full-Scale Bearing (B1) Test}

Figure 2.11 shows load and head velocity versus time during the failure process. Each velocity peak corresponds to a shim failure. The head velocity at failure was one to two orders of magnitude greater than the head velocity prior to failure. This is consistent with the visual observation that the head movement prior to failure was imperceptible, with downward jumps of the load-head observable as shims failed. 
After the initial shim failure, resistance decreased rapidly. Five shims ruptured during a period of nine seconds, and the resistance decreased from $40 \mathrm{MN}$ to $21 \mathrm{MN}$. The bearing then recovered to $22 \mathrm{MN}$, whereupon another series of ruptures followed. This pattern was repeated once more before the test was terminated.

\subsubsection{1/2-Scale Bearing (B2) Test}

\subsubsection{Visual Observations and Load vs Displacement Curve for $\mathbf{1} \mathbf{2}$-Scale Bearing (B2) Test}

During loading to failure, bulging became noticeable at a compressive stress of about $40 \mathrm{MPa}$ and a compressive strain of about $0.052 \mathrm{~m} / \mathrm{m}$. The bulging was asymmetric: the bearing bulged significantly on the south and southwest side, and bulged very little on the north and west side. This bulging pattern continued up through failure. Failure occurred at $88.7 \mathrm{MPa}$ compressive stress and a compressive strain of $0.227 \mathrm{~m} / \mathrm{m}$, and was initiated by a shim rupture at or near mid-height, followed by a series of ruptures successively further away from mid-height.

Figure 2.12 shows initial shim yielding at a compressive stress of about 45 $\mathrm{MPa}$ and a compressive strain of about $0.06 \mathrm{~m} / \mathrm{m}$. Similar to the Full-Scale Bearing (B1), the shape of the stress-strain curve up to this point is concave-upward, whereas between initial yield and the first shim rupture the shape is concave-downward.

\subsubsection{Head Velocity and Load During Failure for 1/2-Scale Bearing (B2) Test}

Figure 2.13 shows load and head velocity versus time during the failure process. Each velocity peak corresponds to a shim failure. The head velocity at failure was one to two orders of magnitude greater than the velocity prior to failure. This is consistent with the visual observation that the head movement prior to failure was imperceptible, with downward jumps of the load-head observable as shims failed.

After initial failure, load fell quickly. Over fifteen seconds, there were at least five shim ruptures, and the resistance decreased from $10.7 \mathrm{MN}$ to $5.8 \mathrm{MN}$. The bearing then recovered to $6.8 \mathrm{MN}$, whereupon there were another series of ruptures.

\subsubsection{1/4-Scale Bearing (B3) Test}

\subsubsection{Visual Observations and Load vs Displacement Curve for 1/4-Scale Bearing (B3) Test}

Bulging was first noticeable at a compressive stress of about $20 \mathrm{MPa}$ and a compressive strain of about $0.067 \mathrm{~m} / \mathrm{m}$. Bulging increased steadily to failure, at a compressive stress $96.7 \mathrm{MPa}$ and a compressive strain of $0.281 \mathrm{~m} / \mathrm{m}$. Bulging appeared to be uniform around the circumference of the bearing. Bulging was greatest at the mid-height and the shape was parabolic when viewed from the side. Initial shim failure was not audible. It appeared that shim rupture was at or near the mid-height of the bearing, with subsequent failures occurring successively further from mid-height.

In this test, load application was continued considerably beyond failure of all shims. The load carrying capacity eventually increased because after the failure of all shims, the bearing essentially acted as a single, unbonded layer of elastomer (even 
bond to the end plates was eventually lost). By the end of the test compressive strains exceeded $0.75 \mathrm{~m} / \mathrm{m}$.

Figure 2.14 shows initial shim yielding at a compressive stress of about 52 $\mathrm{MPa}$ and a compressive strain of about $0.11 \mathrm{~m} / \mathrm{m}$. Similar to the Full and $1 / 2$-Scale Bearings, the shape of the stress-strain curve up to this point is concave-upward, whereas between initial yield and the first shim rupture the shape is concavedownward.

\subsubsection{Head Velocity and Load During Failure for 1/4-Scale Bearing (B3) Test}

Figure 2.15 shows load and head velocity versus time during the failure process. Each velocity peak corresponds to a shim failure. The head velocity at failure was one order of magnitude greater than the velocity prior to failure. This is consistent with the visual observation that the head movement prior to failure was imperceptible, with downward jumps of the load-head observable as shims failed.

After initial failure, load fell quickly. Over thirty seconds, there were at least five shim ruptures, and the resistance decreased from $2.75 \mathrm{MN}$ to $1.70 \mathrm{MN}$. The bearing then recovered to $1.85 \mathrm{MN}$, whereupon there were another series of ruptures.

\subsubsection{Comparisons among A and B-Bearing Tests}

Figure 2.16 shows the stress-strain curves of all specimens on the same plot (the first cycle of loading to $53.4 \mathrm{MN}$ for the A-Bearing test is shown). Note the similarity between the three curves for the three B-Bearings, in spite of the fact that the bearings were not strictly geometrically similar (c.f. Table 2.1). All curves of the B-Bearings have similar slopes, except at low stress values. Differences in the initial stiffnesses may be due in part to misalignment of the load-head and endplate. Measurement of any misalignment is not possible, since, as shown in Figure 2.6, and as discussed in Section 2.5, the LVDTs were not attached to the bearings, but instead contacted the load head directly.

The approximate tangent stiffness of the A-Bearing is $1700 \mathrm{MPa}$ just prior to yield. The corresponding stiffness for the B-Bearings is $930 \mathrm{MPa}$. Bearing $A$ had a rubber layer shape factor of 24.4 , whereas the B-Bearings had a shape factor of 15 . A higher shape factor results in a higher compressive stiffness for the A-Bearing. Similar observations have been reported by researchers of bridge elastomeric bearings (Minor and Egen, 1970; Stanton and Roeder, 1982; Roeder et al., 1987).

If the lower initial stiffnesses of the Full-Scale and 1/4-Scale Bearings were indeed due to plate and head misalignment, the stress-strain curves can be shifted horizontally. In Figure 2.17, the 1/4-Scale stress-strain and Full-Scale stress-strain curves were shifted to the left by strains of .037 and .005 respectively, in order to clearly show the nearly identical slope of the stress-strain curves just prior to initial shim yielding. However, the stress-strain curves, while similar in shape, are clearly not identical. For example, the maximum stress at failure for the Full-Scale Bearing is $90 \mathrm{MPa}$; for the $1 / 2$-Scale Bearing the maximum stress is $88.7 \mathrm{MPa}$, or $1.4 \%$ smaller, and for the 1/4-Scale Bearing the maximum stress is $96.7 \mathrm{MPa}$, or $7.4 \%$ larger. Also, the strain at failure for the Full-Scale Bearing is 0.267 , but is 
$0.227(-13.0 \%)$ for the $1 / 2$-Scale Bearing, and $0.281(+6.5 \%)$ for the $1 / 4-$ Scale Bearing. Finally, there is a clear trend toward higher stress and strain at the point of initial yielding as we move from the Full-Scale Bearing down to the 1/4-Scale Bearing.

\subsection{Measurement Accuracy and Variability}

The accuracy of the Universal Testing Machine machine is established through a calibration using known dead loads, and was calibrated according to ASTM E4, which requires that the machine reading be within $\pm 1 \%$ of the true reading. The machine has met these requirements. This is equivalent to a relative expanded uncertainty $(\mathrm{k}=2)$ of $1 \%$, and is small compared to specimen variability.

The bearing testing program consisted of only one test of each size of bearing, and the stresses at bearing failure, from lowest to highest, are 88.7,90.0, and 96.7 MPa for the 1/2-Scale, Full-Scale, and 1/4-Scale Bearings, respectively. The failure stresses range from $-1.4 \%$ to $+7.4 \%$ of the median value, so the variability in these measurements can be attributed to factors outside the resolution accuracy of the test machine.

Sources of variation of failure stresses include (1) deviations from perfect geometric similarity, (2) differences in the steel and rubber properties, and (3) imperfect manufacture. In Section 6.5, the effect of geometric dissimilarities on bearing response, assuming identical steel and rubber properties, is demonstrated through finite element analysis. Results of steel tensile coupon tests, summarized in Section 5.3, show higher values for the modulus of elasticity and stresses in the plastic range for the 1/4-Scale shims ( 22 gage), and the effect on bearing response is discussed in Section 6.5. The effects of rubber property variability have not been determined in this study (c.f. Section 3.3.5), nor have the effects of imperfect manufacture. 


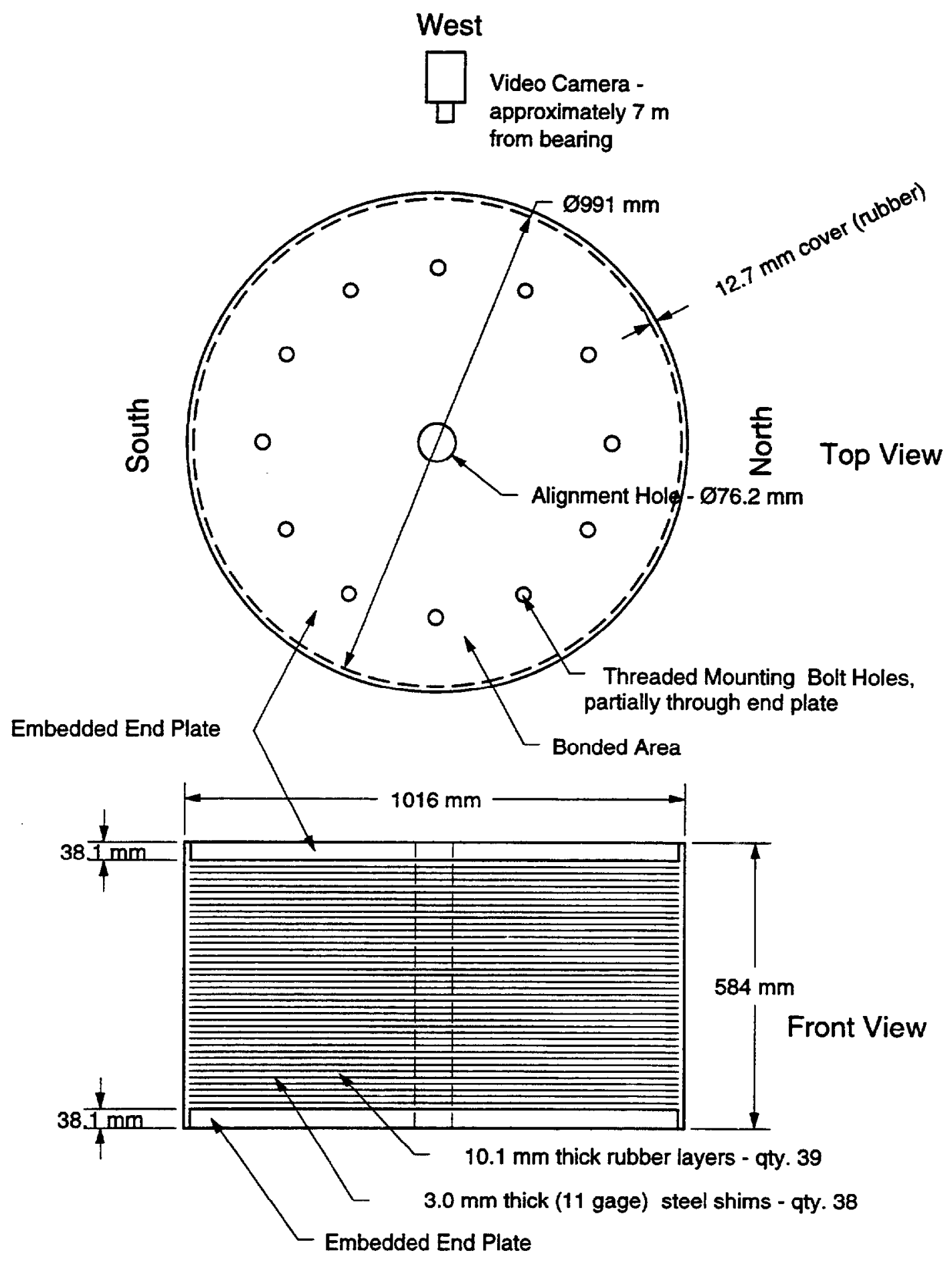

Figure 2.1 - Bearing A dimensions 


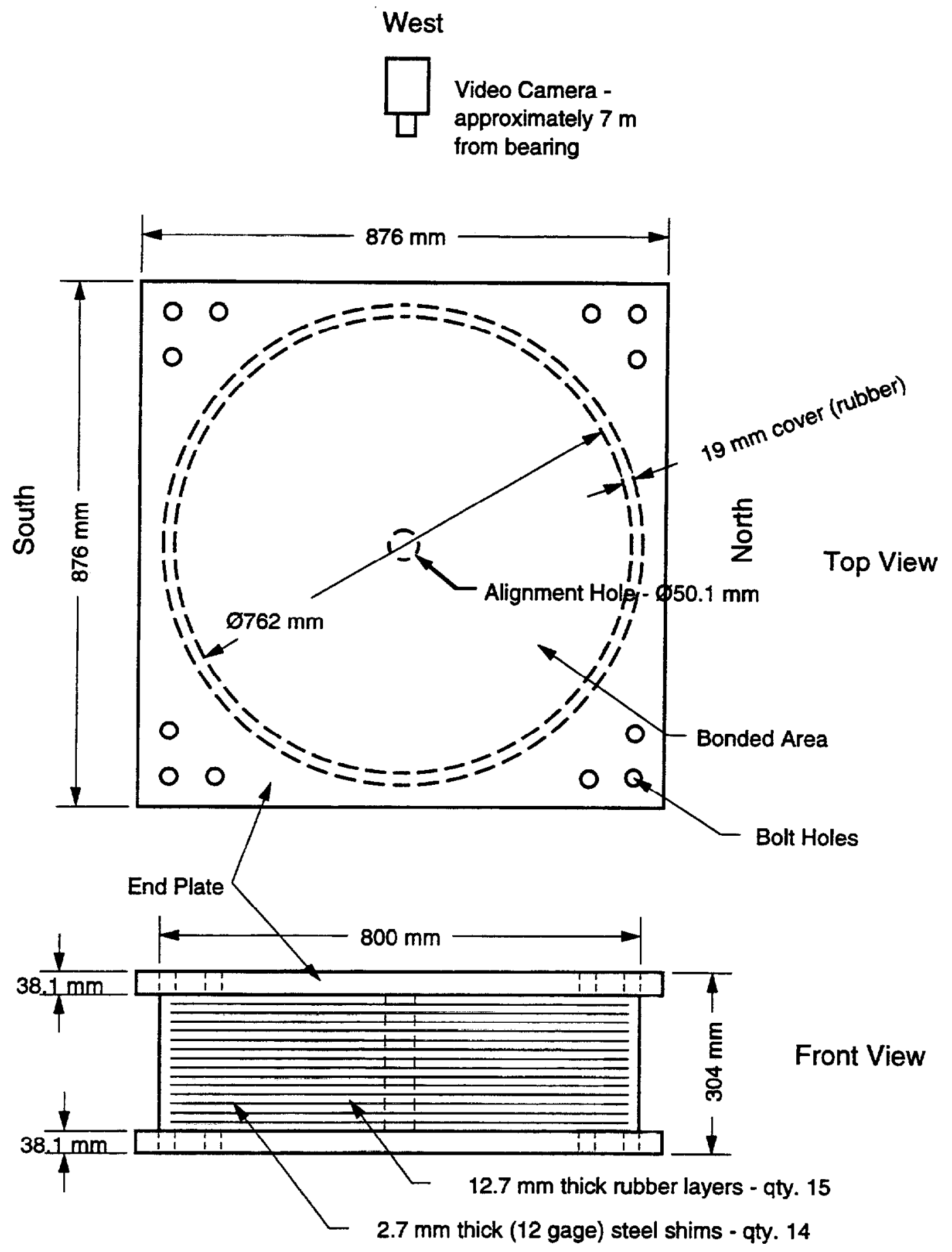

Figure 2.2 - Full-Scale Bearing (B1) dimensions 


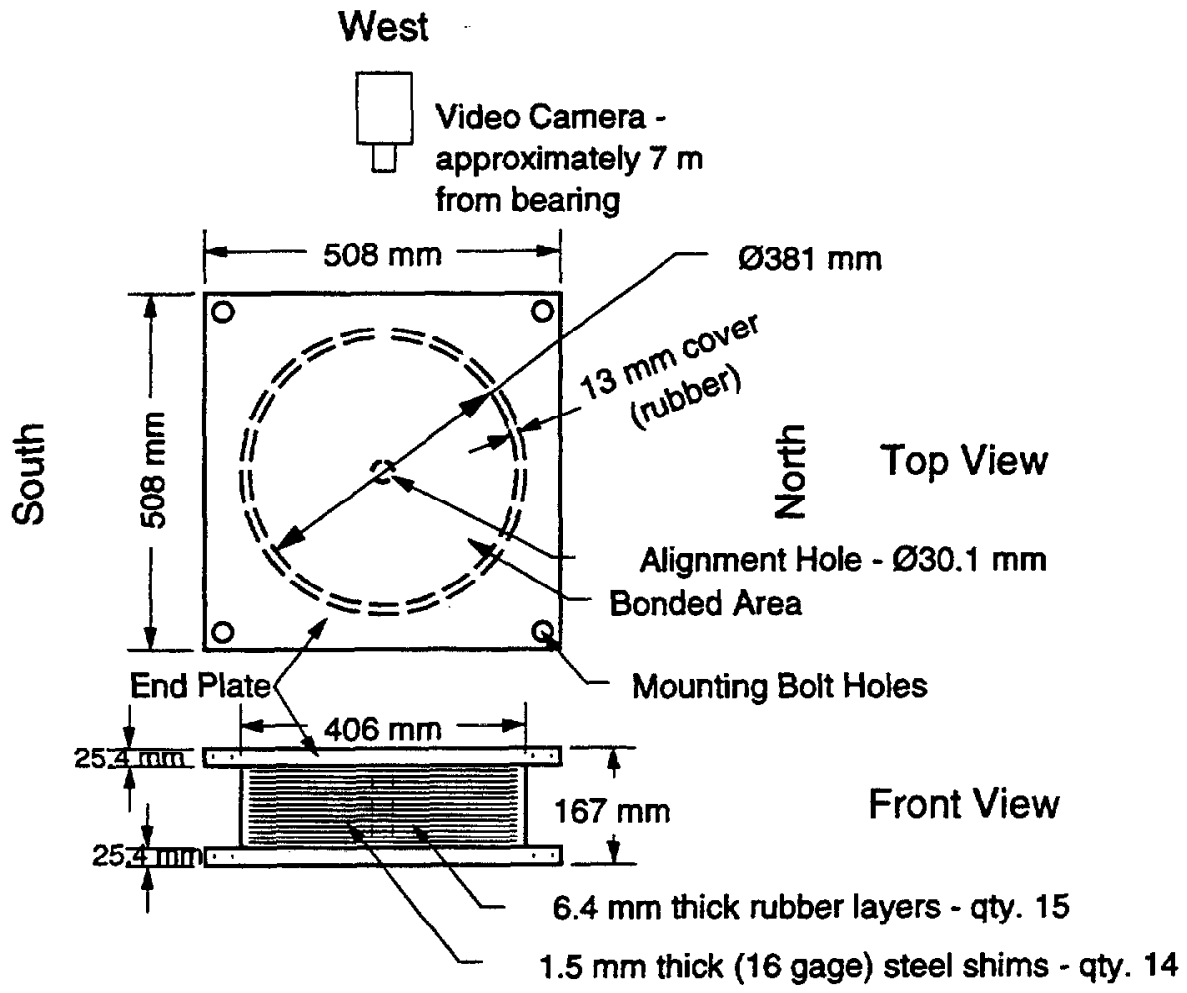

(a) 1/2-Scale Bearing (B2) dimensions
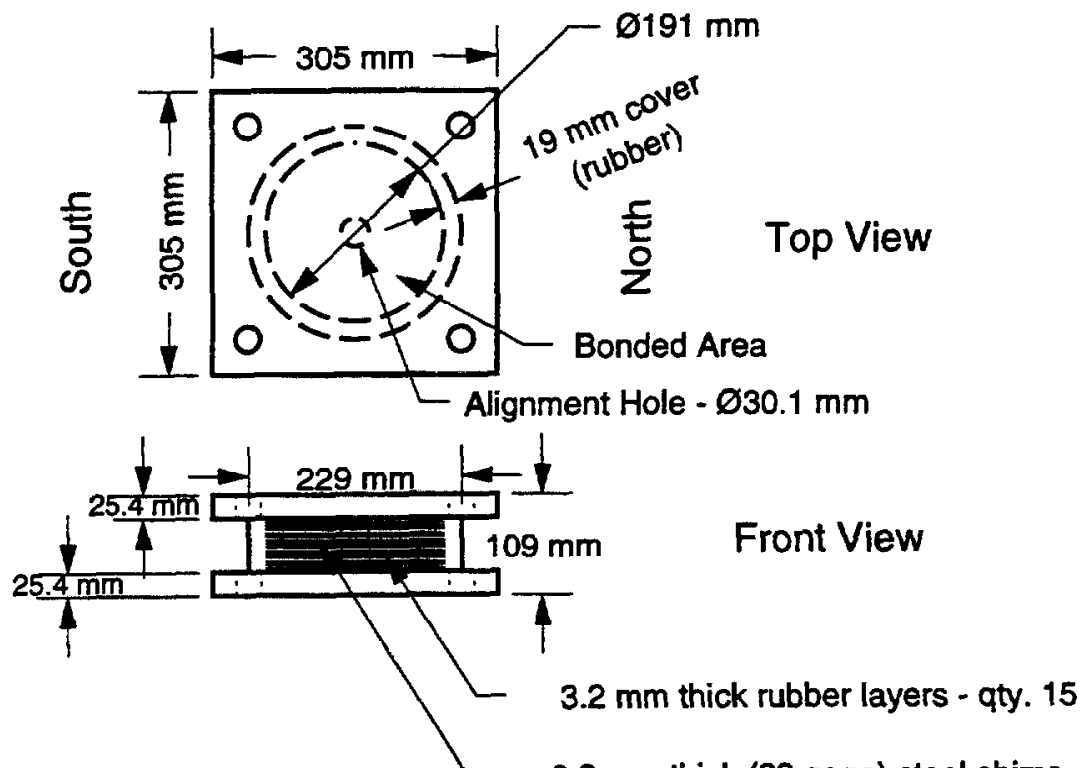

$0.8 \mathrm{~mm}$ thick (22 gage) steel shims - qty. 14

(b) 1/4-Scale Bearing (B3) dimensions

Figure 2.3 - Bearing dimensions for 1/2-Scale (B2) and 1/4-Scale (B3) Bearings 


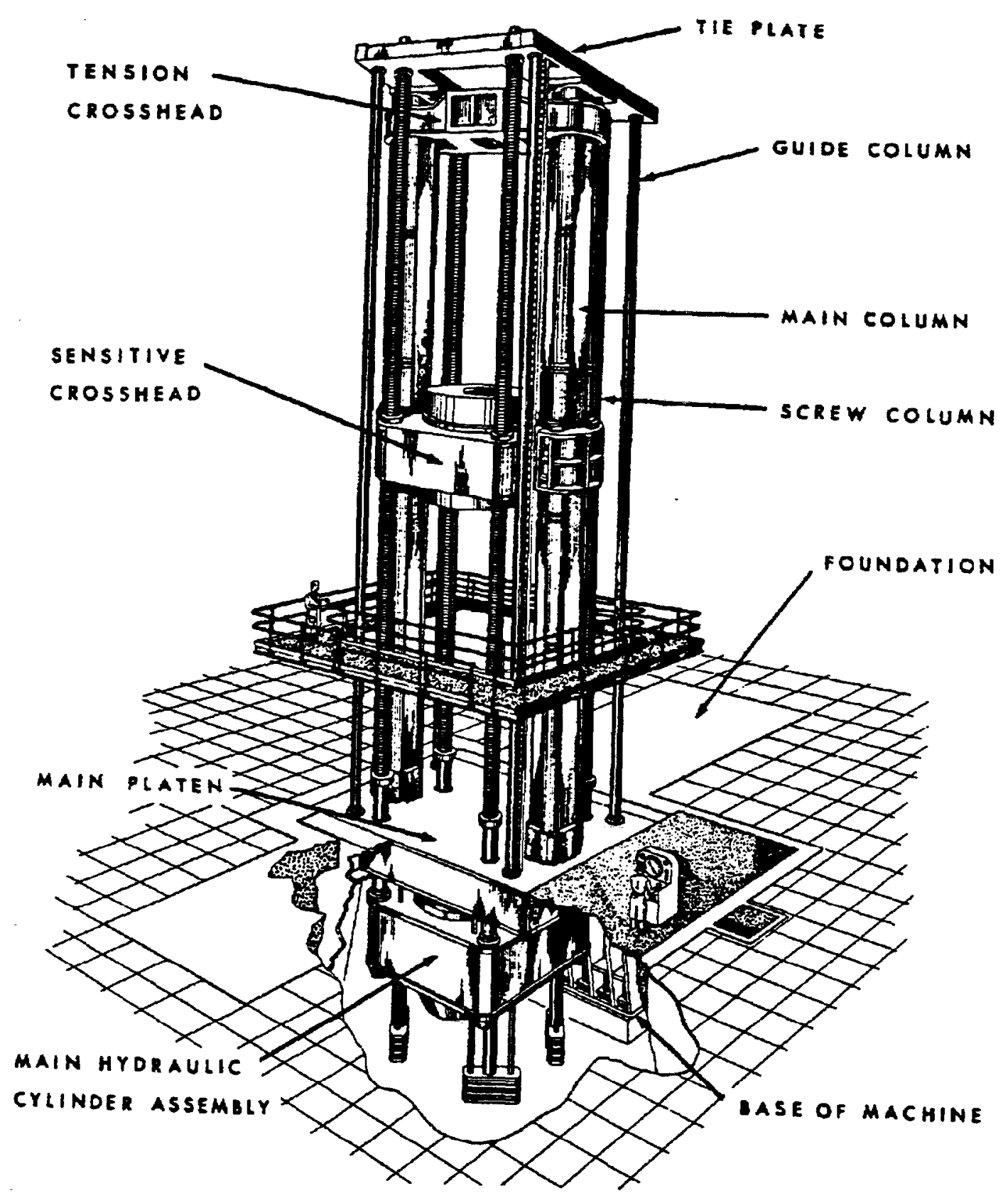

Figure 2.4 - Test machine 


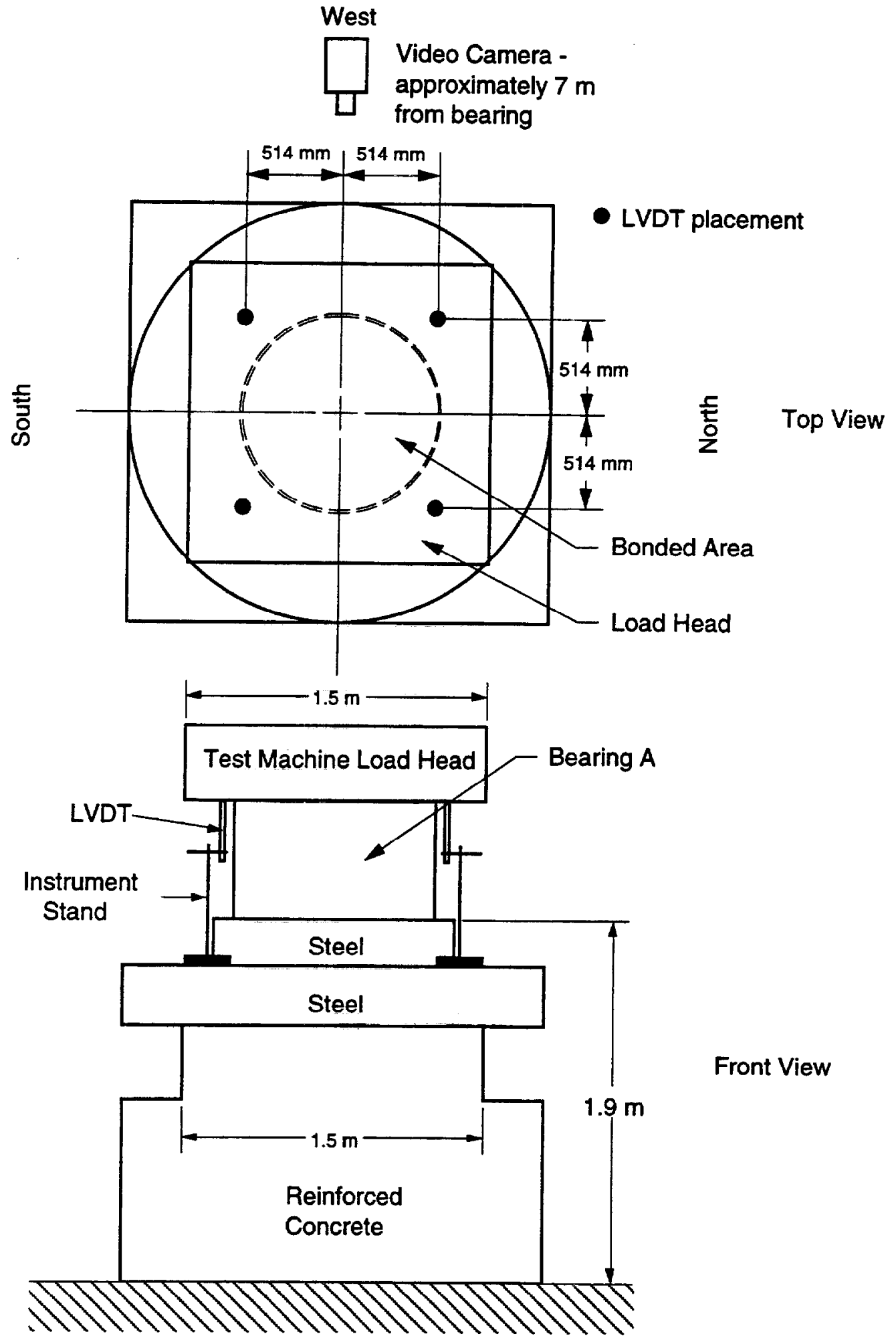

Figure 2.5 - Setup for Bearing A tests 


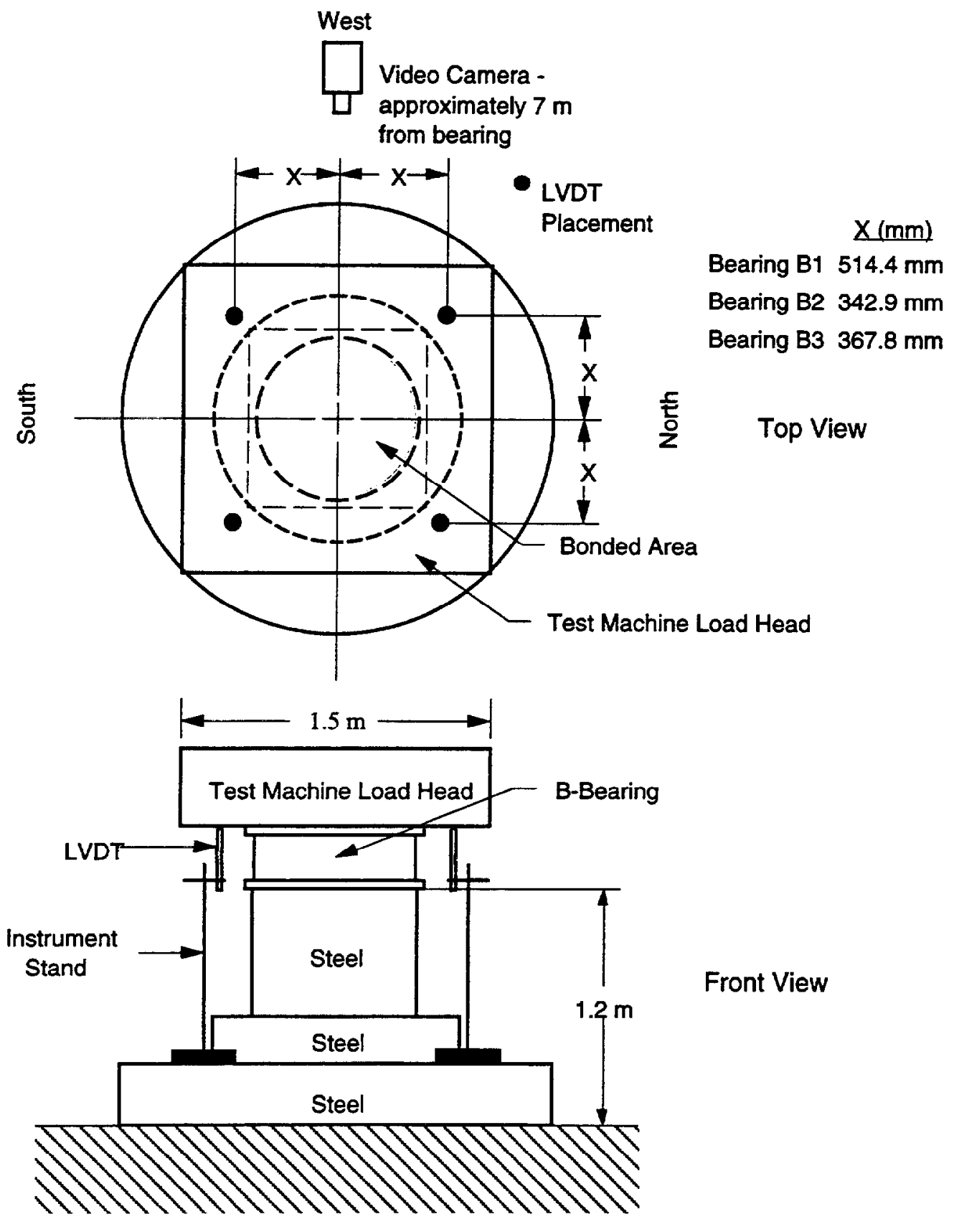

Figure 2.6 - Setup for geometrically similar bearings (B) tests 


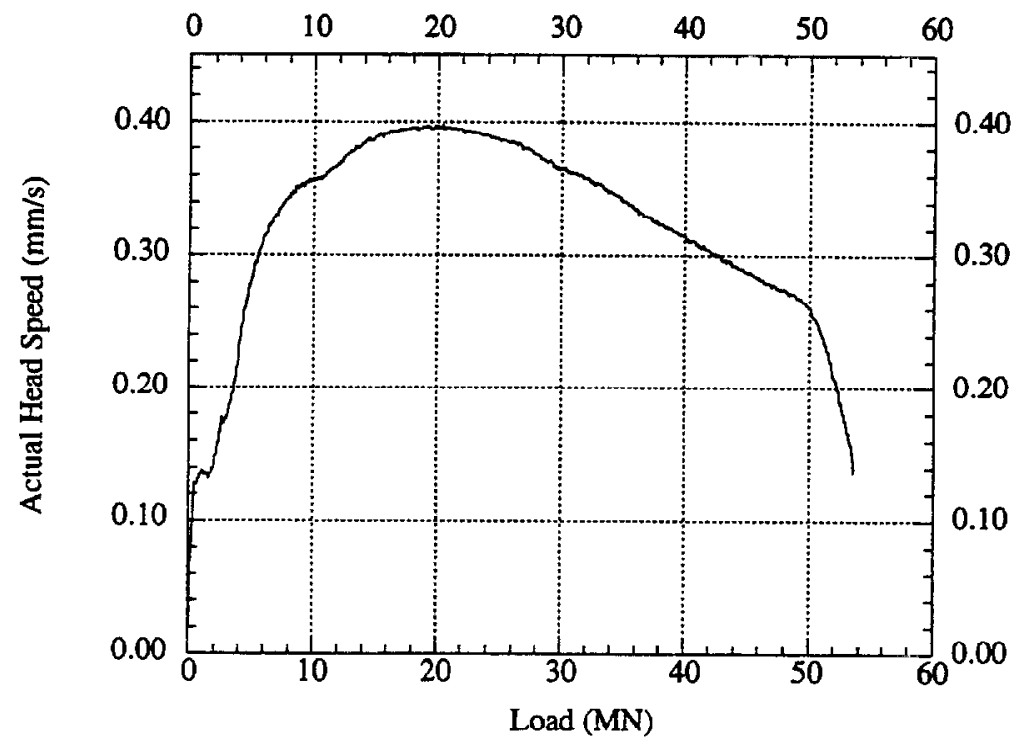

Figure 2.7 - Load-head speed vs load for Bearing A, initial test (2nd cycle)

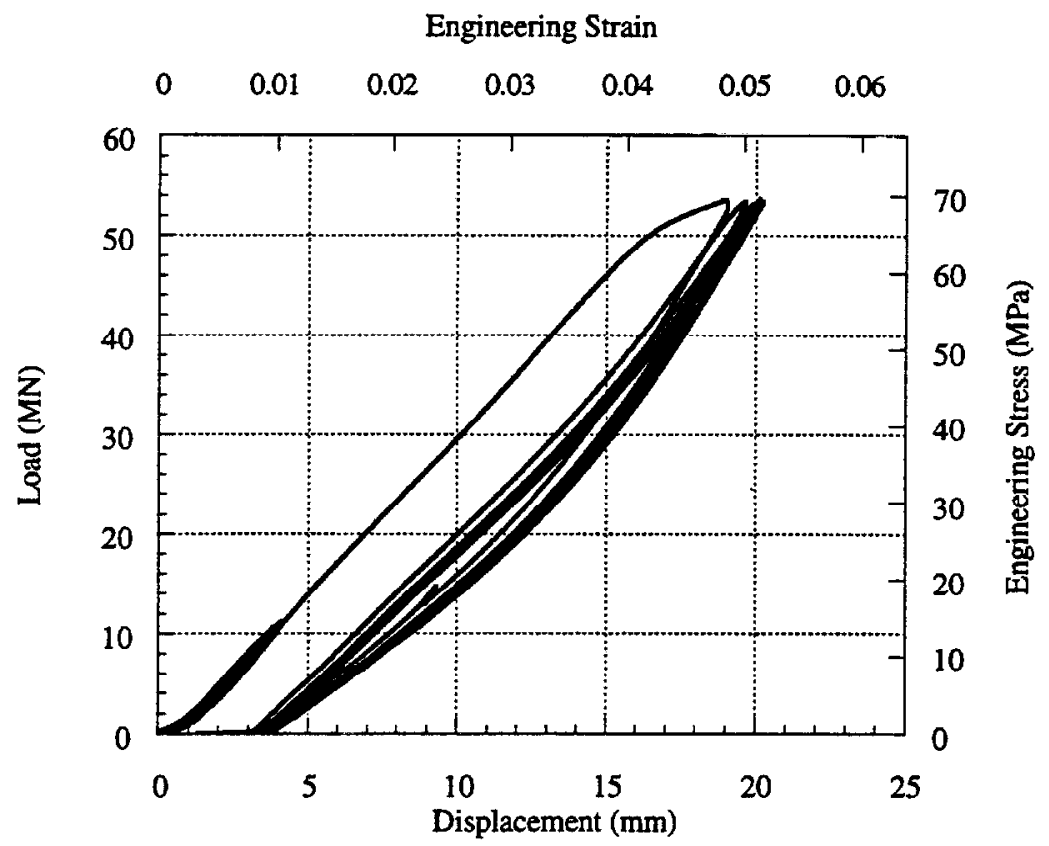

Figure 2.8 - Load vs displacement plot for Bearing A, first test 


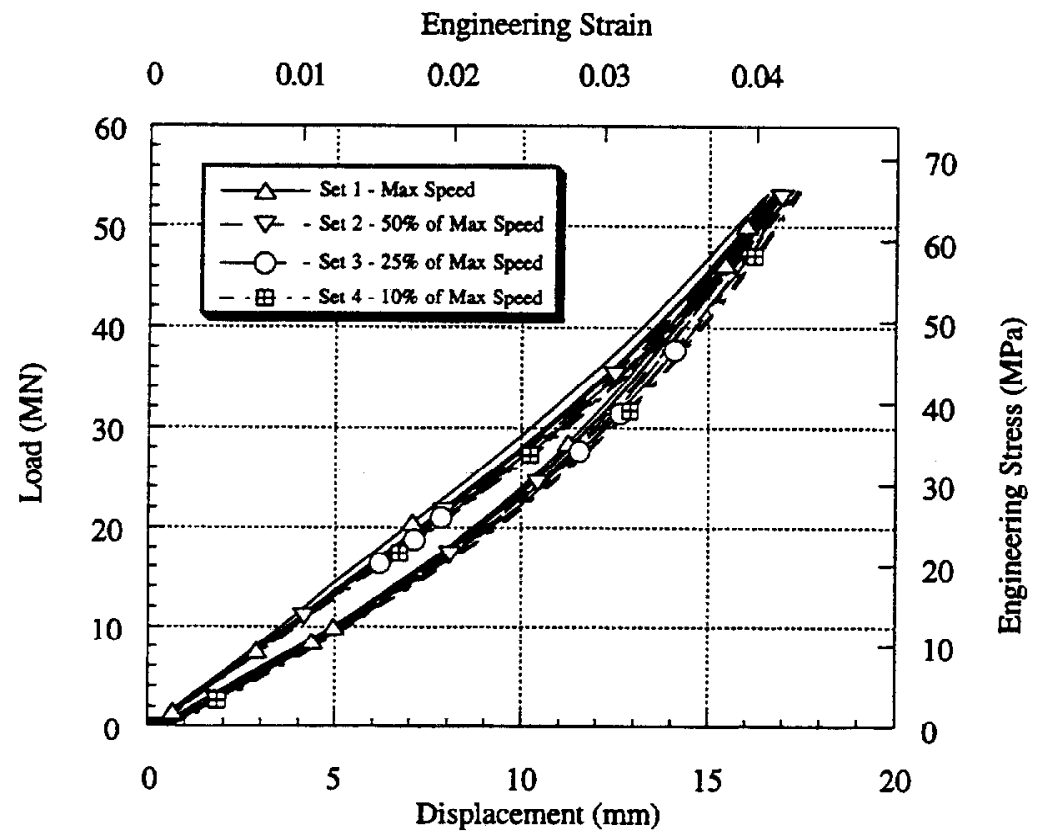

Figure 2.9 - Load vs displacement plot for Bearing A, second test

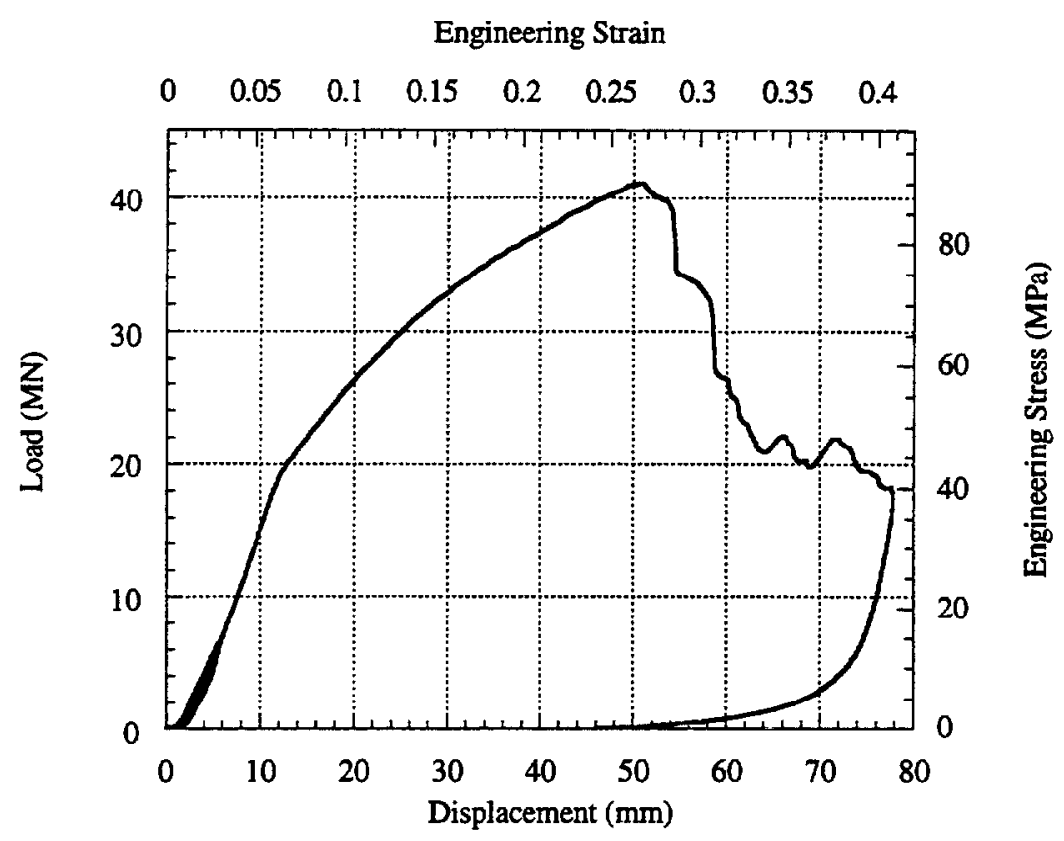

Figure 2.10 - Load vs displacement plot for Full-Scale Bearing (B1) 


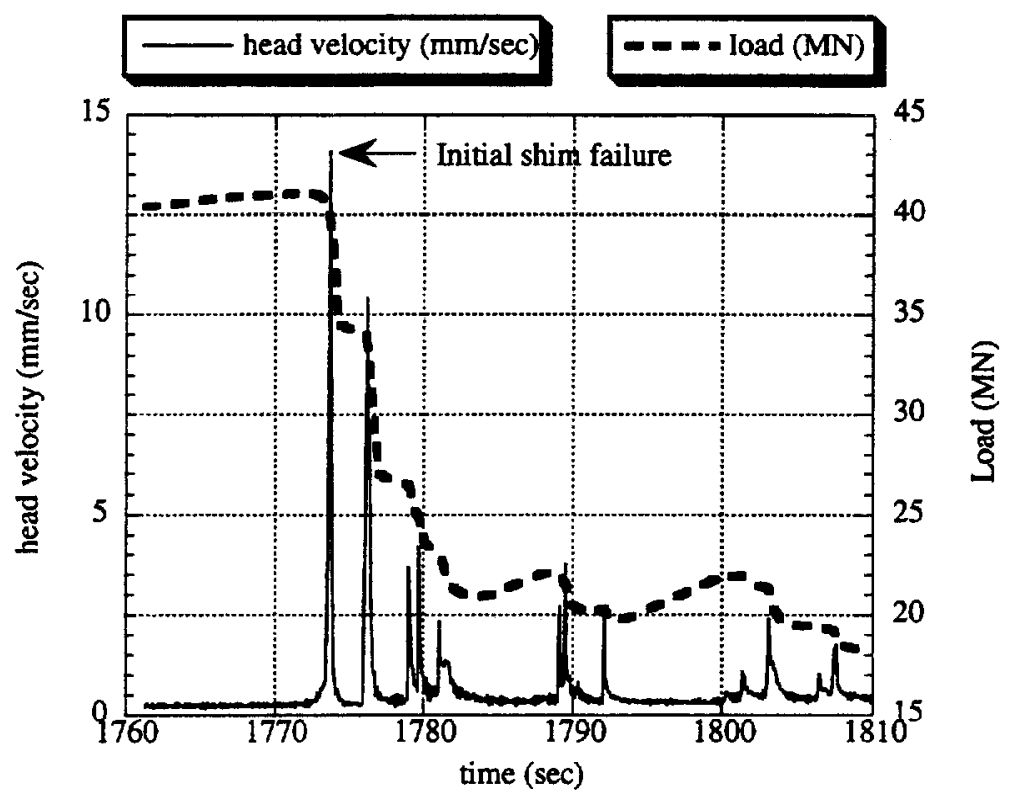

Figure 2.11 - Head velocity and load during shim failures for Full-Scale Bearing (B1)

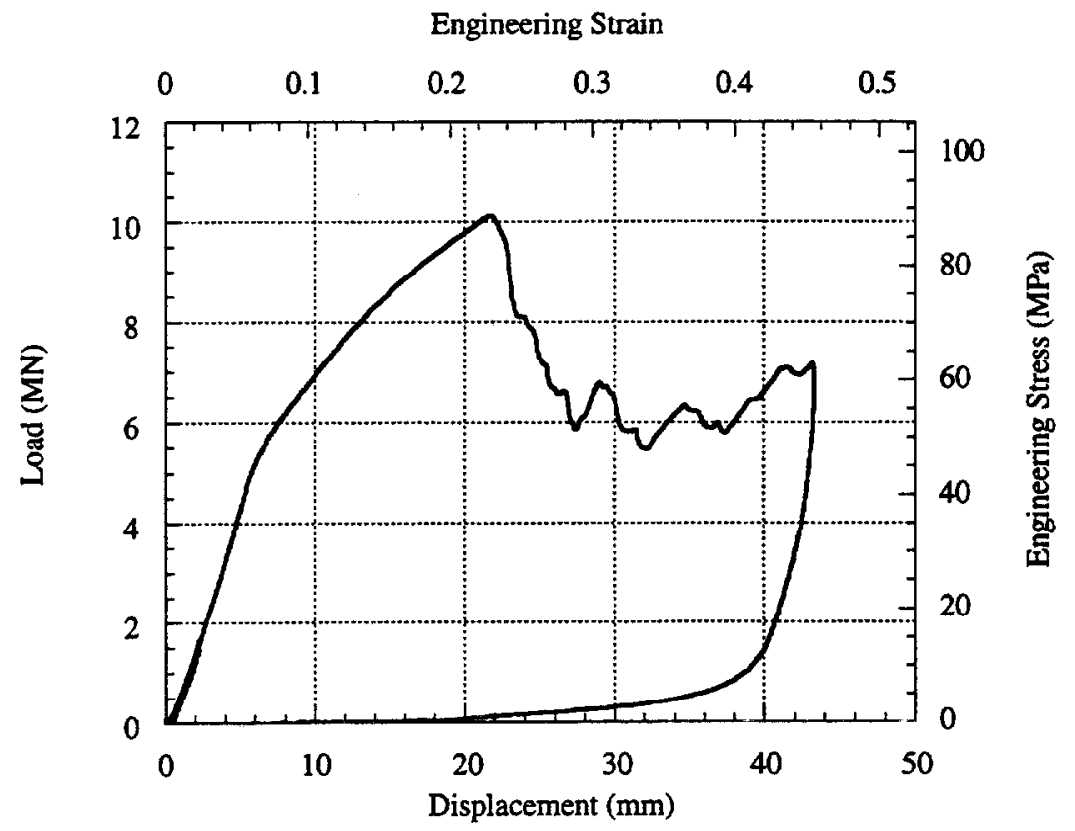

Figure 2.12 - Load vs displacement plot for 1/2-ScaleBearing (B2) 


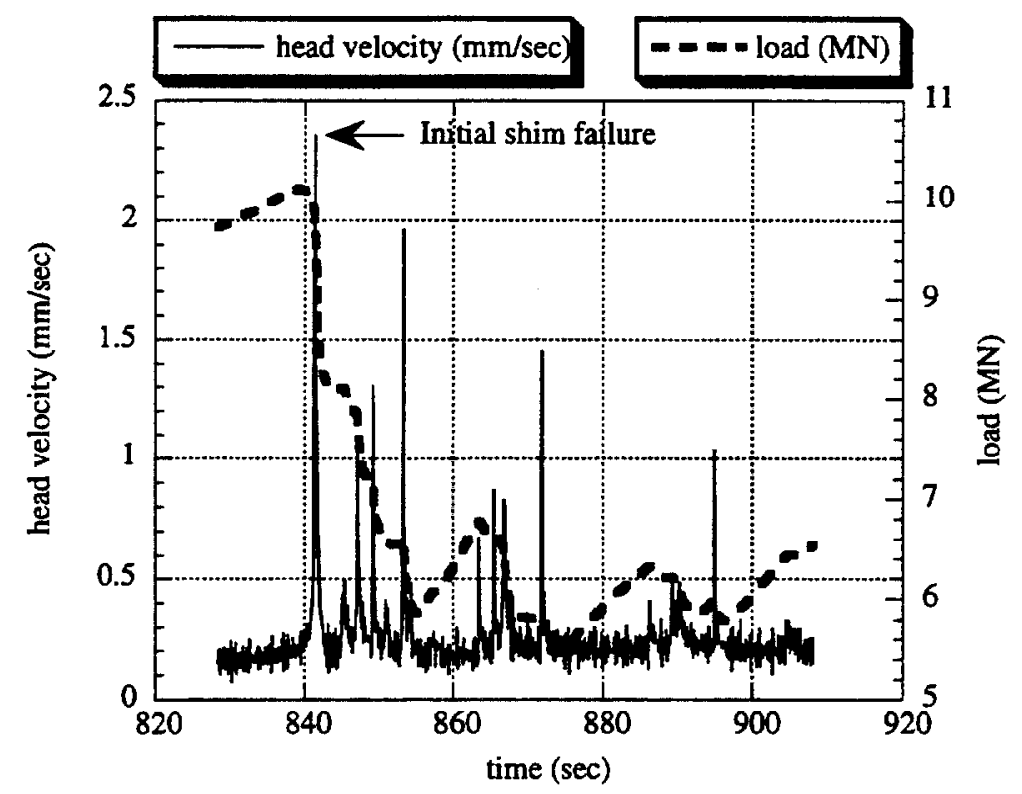

Figure 2.13 - Head velocity and load during shim failures for $1 / 2$-Scale Bearing (B2)

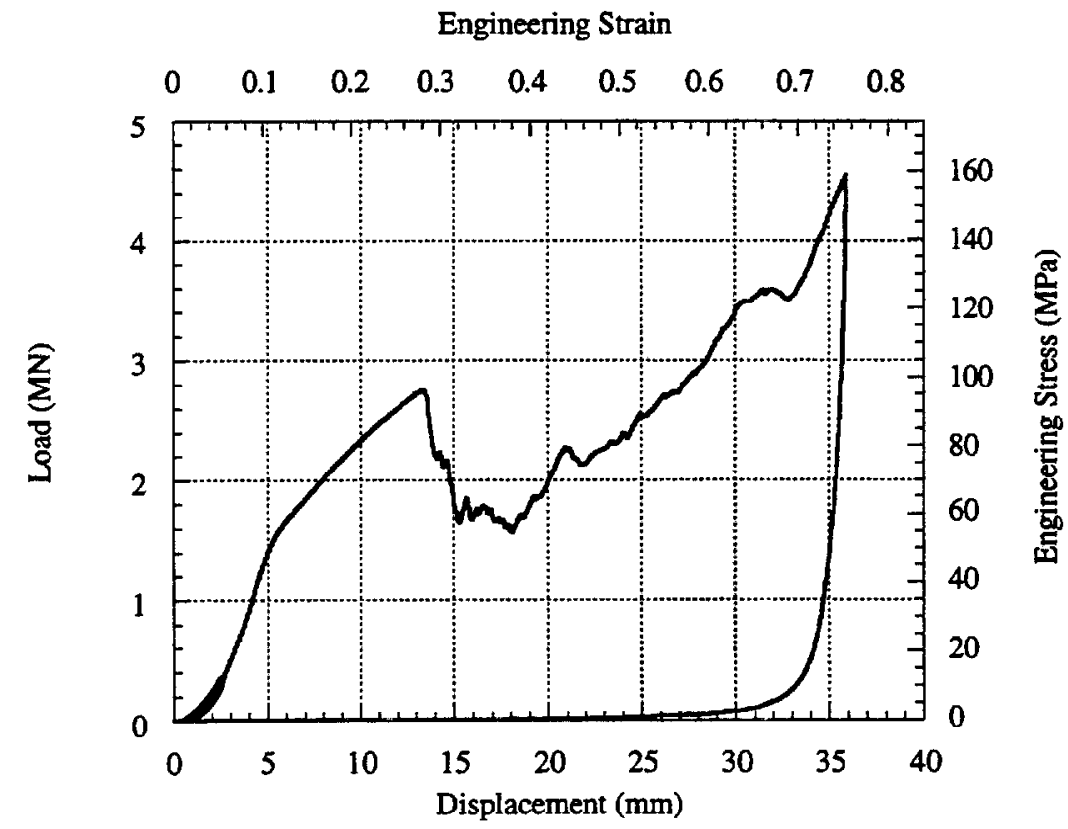

Figure 2.14 - Load vs displacement plot for 1/4-Scale Bearing (B3) 


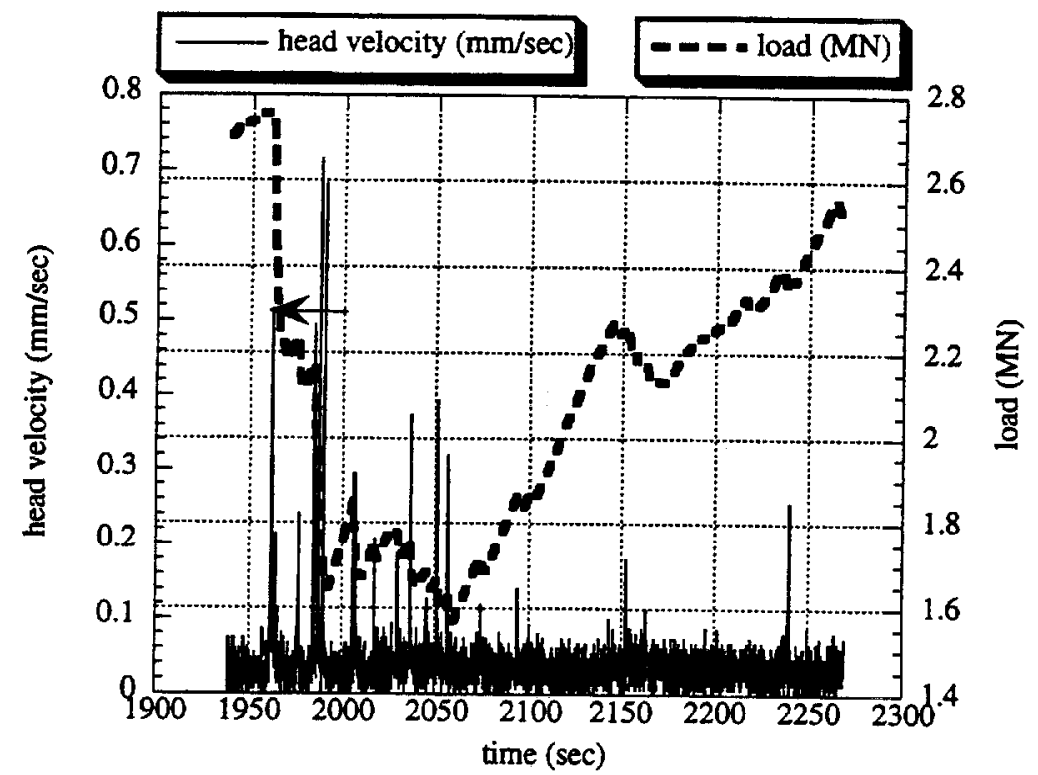

Figure 2.15 - Head velocity and load during shim failures for 1/4-Scale Bearing (B3)

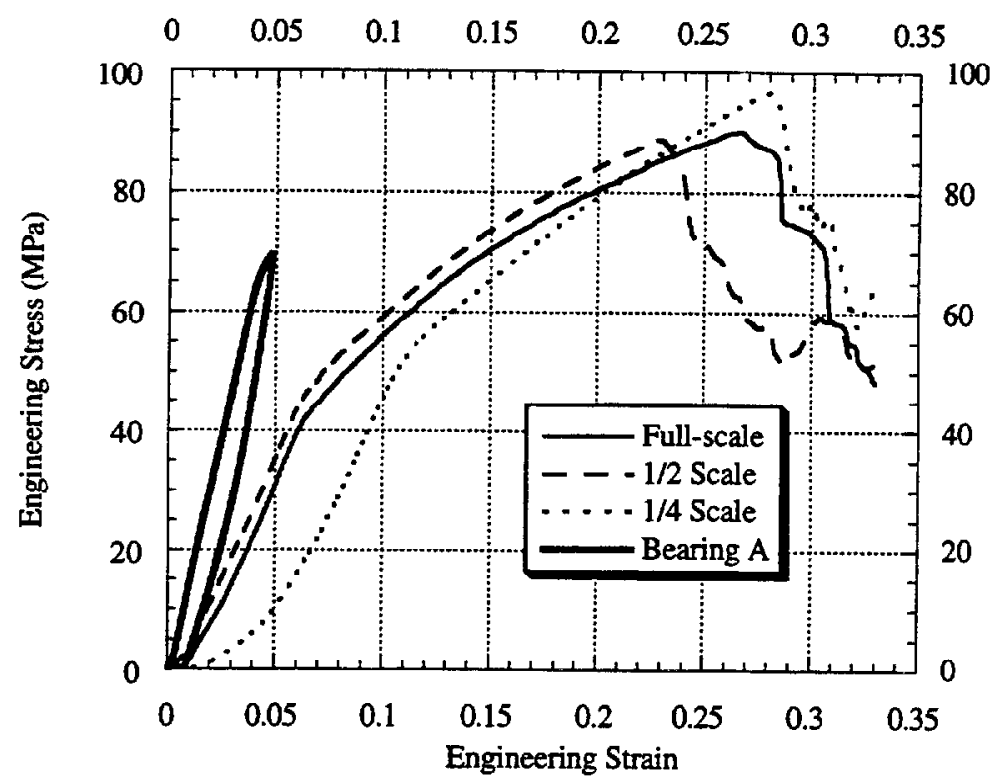

Figure 2.16 - Comparison of stress-strain curves for all bearings 


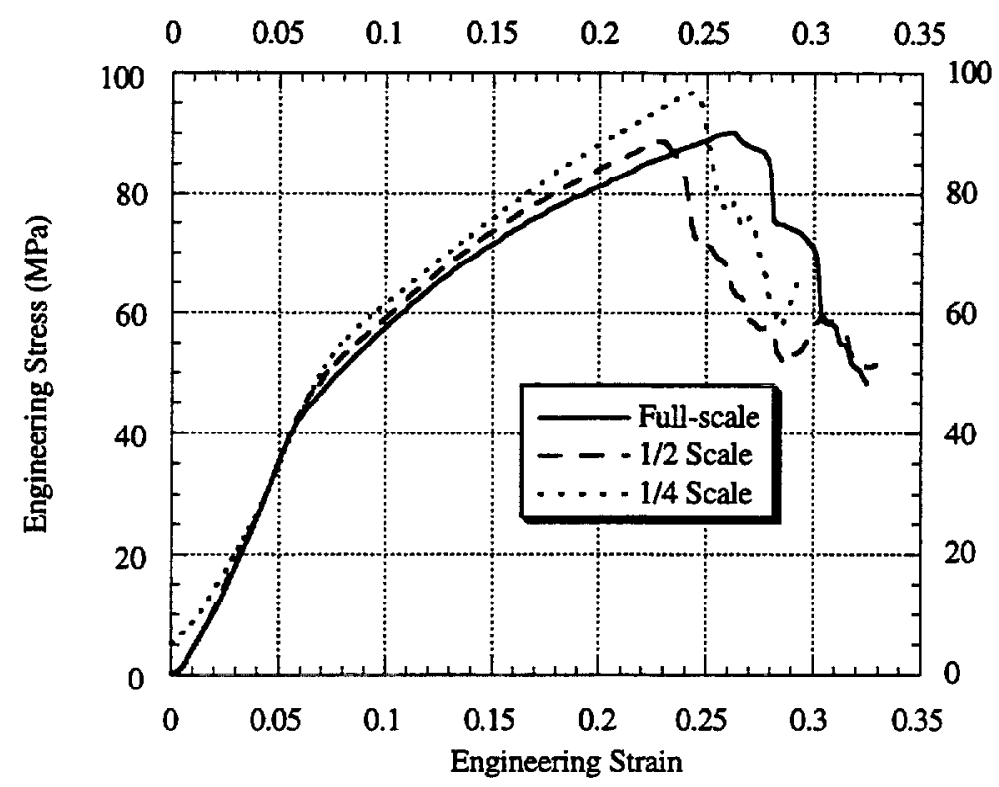

Figure 2.17 - Comparison of adjusted stress-strain curves for B-Bearings 


\section{BLANK PAGE}




\section{DETERMINATION OF RUBBER STRESS-STRAIN PROPERTIES FOR FINITE ELEMENT MODELING}

\subsection{Background for Finite Element Modeling}

Finite element analyses have been conducted on the B-Bearings to investigate the effect of the geometric dissimilarities on the performance of the scale-model bearings, and their abilities to predict the ultimate load capacity.

As noted in Section 2.3.3, each bearing was subjected to at least six mechanical conditioning cycles to $13.79 \mathrm{MPa}$ before they were loaded to failure. During these cycles, the bearing deformation was not elastic. Figure 3.1 shows a tensile load-displacement plot of a dumbbell rubber specimen, where there were three cycles to $29.4 \mathrm{~N}$. The plot shows a reduction in load at each strain level for the second and subsequent cycles, and the curve takes the shape of the initial loading cycle when the extension is continued beyond the extension reached in each cycle. A similar response is present in compressive loading. In the actual bearing tests, the conditioning cycles took the bearings to only $10 \%$ of the failure strain and $15 \%$ of the failure stress. Modeling the response of the bearing to the conditioning cycles would have no impact on capturing the initial yielding of the shims, nor on the response of the bearing in the failure range. Therefore, this effect was not modeled.

The response of rubber is also a function of strain rate and temperature (Treloar, 1975). However, we are not considering these effects in this analysis. Therefore, a hyperelastic model was employed to characterize the behavior of the rubber. In Section 3.2, the constitutive theory and finite element modeling of rubber samples are discussed in detail. In Section 3.3, the experimental testing methods and experimental results are presented (uniaxial tension, uniaxial compression, and volumetric testing). In Section 3.4, extension of the experimental data to other modes of deformation is presented in an attempt to improve the characterization of the strainenergy function. The calibration of the rubber model, e.g. how many terms are to be included in the strain energy function, and whether the extension of the data to other deformation modes improved the modeling, is discussed in Section 4.

Steel samples of 12,16 , and 22 gage thicknesses (c.f. Table 2.1) were tested in the uniaxial tensile mode. In Section 5, the experimental steel testing and modeling will be discussed in detail. Finally, in Section 6, the results of the finite element modeling of the bearings are presented.

The finite element code ABAQUS was used to model the B-Bearings responses to uniaxial compressive loading. ABAQUS is a general purpose nonlinear finite element program that has constitutive models which are appropriate to the analysis. A hyperelastic model is available in order to model rubber-like compounds, including the ability to model slight compressibility of rubber. The model requires nominal stress-strain data of the rubber. For modeling the steel, an elastic-plastic model with strain-hardening was needed, since uniaxial tests of steel samples indicated this type of response. ABAQUS provides a model with these characteristics, and it requires true (Cauchy) stress and logarithmic strain. Therefore, experimental steel data were obtained using these measures.

The input file for ABAQUS is divided into two parts - (1) the model data and (2) the history data. The model data define the geometry of the model, the type or 
types of elements used, and the definitions of the materials. The history data describes how the loads and displacements are prescribed. ABAQUS allows multiple steps to be defined, where each step may be a particular set of loads or displacements, and the initial condition of a step is the solution of the previous step.

To model the simple monotonic loading of the tests, only one step was required in the history data. The history of the step was defined by specifying the vertical downward displacement of the top nodes to be $30 \mathrm{~mm}$ for the prototype, 15 $\mathrm{mm}$ for the 1/2-Scale Bearing, and $7.5 \mathrm{~mm}$ for the 1/4-Scale Bearing, which corresponds to an engineering compressive strain of $0.314 \mathrm{~m} / \mathrm{m}$. In the experimental tests, the compressive strain at failure ranged from $0.227 \mathrm{~m} / \mathrm{m}$ for the $1 / 2$-Scale Bearing to $0.281 \mathrm{~m} / \mathrm{m}$ for the 1/4-Scale Bearing. A single node along the top was designated as the master node, and the vertical displacement of all other nodes along the top were tied to the vertical displacement of the master node. This was convenient, since by defining the displacements this way, the reaction forces for all top nodes were summed at the master node.

Since the rate of loading in the experiments was very slow, inertial effects were negligible. Therefore, a static stress analysis was performed. This type of analysis is specified in ABAQUS by using the STATIC keyword. In addition, an arclength control method was used. In arc-length control, the distance traveled along the equilibrium path is specified, and the actual load value is unknown at the outset. Without the use of the arc-length control method, convergence could not be obtained beyond approximately $4 \%$ of the desired vertical displacement. Successful convergence was obtained for the entire vertical displacement with the use of arclength control. The arc-length control method is specified in ABAQUS by selecting the keyword RIKS. For further information on these topics, consult the ABAQUS/Standard Users Manual (1995).

\subsection{Theoretical Basis for Constitutive Modeling of Rubber}

Rubber consists of many interwoven, long chain polymers that initially (prior to loading) are randomly oriented; therefore, rubber can be assumed to be isotropic. Also, rubber exhibits a nonlinearly elastic response up to large strains, i.e. a loaded specimen will return nearly to its unloaded state after being stretched or compressed a great amount (for rubber, the tensile strains can exceed $600 \%$, and the compressive strains can exceed $85 \%$ ). Consequently, a hyperelastic model was used to characterize the rubber behavior.

A hyperelastic model associates a state of strain to a unique state of stress ("hyper" means that the model is elastic over a large strain range). The strain energy potential can be defined at each point in the material based on this stress-strain relationship, and stresses can be found directly by taking appropriate derivatives of the strain energy potential. Since the material is assumed to be isotropic, the strain energy potential can be written as a function of the strain invariants, $U=U\left(I_{1}, I_{2}, I_{3}\right)$, where $I_{3}$ is the square of the volume ratio $\left(\sqrt{I_{3}}=J\right)$ and is equal to 1 for a perfectly incompressible material (see Appendix A for a description of the invariants of the deformation tensor used in ABAQUS). 
In general, the total stress at each point in a body can be found if the displacements are known. However, if a material is perfectly incompressible, the hydrostatic stress $\left(\frac{t_{11}+t_{22}+t_{33}}{3}\right)$, where $t$ represents true stress, cannot be found from the displacements, since the application of a hydrostatic stress results in no deformation. "Mixed" formulations have successfully dealt with this problem (Peng and Chang, 1997; Chen and Pan, 1996). In a mixed formulation for a perfectly incompressible material, the internal energy is augmented by adding the term $p(J-1)$, where $p$ is a Lagrange multiplier (the hydrostatic stress) introduced to impose the constraint $J-1=0 \quad(J-1$ is the volumetric strain). This allows the hydrostatic stress to be approximated directly, independently of the displacements. The stress that is derived from the displacements is the deviatoric stress. The sum of the hydrostatic and deviatoric stress tensors gives the total stress tensor.

Good results have been obtained when modeling solid rubber as an incompressible material. A Poisson's Ratio of 0.5 is equivalent to an incompressible material. For solid nubbers, Poisson's Ratio is very close to 0.5 , usually 0.498 and above. For the rubber in the B-bearings, it is estimated to range from 0.4994 to 0.4999 , so it is very close to an incompressible material. This range of values was found from the equation $v=\frac{1}{2}\left(1-\frac{E}{3 K}\right)$, which requires estimates of Young's modulus $\mathrm{E}$ and bulk modulus $\mathrm{K}$. The tests to determine these parameters were performed on rubber samples taken from rubber batches used to produce the B-Bearings, and are discussed in detail in Section 3.3.

The lower value of 0.4994 was found as follows: Young's modulus E was determined by averaging the moduli from uniaxial compressive and tensile tests, and gave a value of approximately $10.77 \mathrm{MPa}$. The bulk modulus $\mathrm{K}$ was determined from a volumetric compression test to be approximately $3250 \mathrm{MPa}$ (the slope of the Pressure versus Volume Ratio curve, Figure 3.13). These values of $\mathrm{E}$ and $\mathrm{K}$ give a Poisson's ratio of 0.4994 .

The upper value of 0.4999 was found by using the value of the shear modulus (G) reported by the bearing manufacturer. The shear modulus was determined to be 128 psi $(0.8825 \mathrm{MPa})$. The bearing manufacturer used the double shear test of Annex A1 of ASTM D 4014-89 (c.f. Table 2.3). Young's modulus E was found from the equation $G=\frac{E}{2(1+v)}$, where a value for the Poisson's ratio of 0.4900 was assumed. The resulting value of Young's modulus, and the value of the bulk modulus discussed above, were used to determine Poisson's ratio from $v=\frac{1}{2}\left(1-\frac{E}{3 K}\right)$. This value was compared to the assumed value of 0.4900 . A second iteration showed Poisson's ratio to be 0.4999 .

Due to the near incompressibility of rubber $(v \approx 0.5)$, when loaded it will tend to maintain its volume and squeeze into any free space available. However, there isn't much free space available for movement of the rubber in our bearings (Shape Factor = 15), except at the exterior of the bearing. Since the rubber is highly confined, the slight 
compressibility of the rubber may be significant in obtaining accurate predictions of the stiffness of the bearings, and therefore could affect the analytical prediction of the deformation and ultimate bearing load (Roeder et al., 1987).

As noted above, the total stress is derivable from the displacements, assuming the material is not incompressible. However, the near incompressibility of solid rubber means that the stiffness matrix becomes nearly singular. This problem is compounded by the approximations inherent in any finite element approach, for example, the calculation of the strains involves taking derivatives of the displacement numerically, resulting in more error. It has been found that a mixed formulation can be utilized effectively in this situation (Peng and Chang, 1997; Chen and Pan, 1996). The general approach is to decouple the strain energy into a purely deviatoric portion and a purely volumetric portion. The deviatoric portion is written using revised invariants that remove any effect due to volume change. The strain energy is written as

$$
U=U\left(\bar{I}_{1}, \bar{I}_{2}, I_{3}\right)=\bar{U}\left(\bar{I}_{1}, \bar{I}_{2}\right)+\tilde{U}\left(I_{3}\right),
$$

where the bars over the first two strain invariants indicate removal of volume changes (see Appendix A).

The decoupling of the deviatoric and volumetric strain energy is in general not valid, but it is required for the use of the mixed approach. It has been shown that a constant bulk modulus is required for the mixed formulation to be a valid approach (Sussman and Bathe, 1987). A constant bulk modulus is equivalent to linearity between pressure and volume ratio. Figure 3.11 shows a plot of pressure versus volume ratio for our rubber, as determined in a volumetric compression test. The curve is initially flat, but this is probably due the rubber specimen not fitting exactly into the testing apparatus, thereby allowing the rubber to flow into the free space. However, the curve is nearly linear beyond that region, and on this basis, the decoupling of the strain energy into deviatoric and volumetric portions, as shown in Equation (3.1), is assumed valid for the rubber tested, and therefore the mixed approach is an appropriate model.

The ABAQUS hyperelastic model follows the mixed approach, and was used in the finite element analysis. Two forms of the strain energy function are provided in ABAQUS. The first is the Polynomial Strain Energy Function:

$$
U=\sum_{i+j-1}^{N} C_{i j}\left(\bar{I}_{1}-3\right)^{i}\left(\bar{I}_{2}-3\right)^{j}+\sum_{i=1}^{N} \frac{1}{D_{i}}\left(J_{e l}-1\right)^{2 i}
$$

The first summation is the contribution due to deviatoric effects, and the second summation is the contribution due to volumetric effects. The $C_{i j}$ and $D_{i}$ are parameters that are found from the rubber test data. $\bar{I}_{1}$ and $\bar{I}_{2}$ are the first and second invariants of the Cauchy-Green Deformation Tensor with the volume change removed, and $J_{e l}$ is the ratio of the current volume to the original volume excluding thermal effects (see Appendix A). For $N=1$, the deviatoric contribution to the strain energy is the MooneyRivlin function, which is $U=C_{10}\left(\bar{I}_{1}-3\right)+C_{01}\left(\bar{I}_{2}-3\right)$.

The strain energy potential function of an isotropic elastic material can also be written in terms of the stretch (extension) ratios $\lambda$. According to Valanis and Landel 
(1967), "A necessary and sufficient condition that $U$ will pertain to an isotropic elastic material is that it merely be a symmetric function of the extension ratios $\lambda_{a}$. Thus, an alternate and equally general form of $U$ could be $U=U\left(\lambda_{1}, \lambda_{2}, \lambda_{3}\right)$." Furthermore, Valanis \& Landel postulate that the strain energy function for rubber can be written as an additive and separable function of the stretch ratios as $U=u\left(\lambda_{1}\right)+u\left(\lambda_{2}\right)+u\left(\lambda_{3}\right)$ $(u(\cdot)$ is termed the Valanis-Landel function).

The second form for the strain energy potential used in ABAQUS is the Ogden Strain Energy Potential. The contribution due to deviatoric effects is written as a function of the stretch ratios, and is functionally identical to the Valanis-Landel form shown above. The contribution due to volumetric effects is the same as that used for the Polynomial Strain Energy Function:

$$
U=\sum_{i=1}^{N} \frac{2 \mu_{i}}{\alpha_{i}^{2}}\left(\bar{\lambda}_{1}^{\alpha_{i}}+\bar{\lambda}_{2}^{\alpha_{i}}+\bar{\lambda}_{3}^{\alpha_{i}}-3\right)+\sum_{i=1}^{N} \frac{1}{D_{i}}\left(J_{e l}-1\right)^{2 i} .
$$

In Equation (3.3), the $\mu_{i}$ and $\alpha_{i}$ are parameters that are found from the rubber test data. The principal stretch ratios $\bar{\lambda}_{k}{ }^{\alpha_{1}}$ have the volume change removed (see Appendix A). To determine the deviatoric parameters $C_{i j}, \mu_{i}$, and $\alpha_{i}$, experimental data can be fitted to one or more of three deformation modes; uniaxial (tension or compression), equibiaxial (tension or compression), and planar (tension or compression) (ABAQUS 1995a). For a right-handed Cartesian coordinate system with axes $x 1, x 2$, and $x 3$, the uniaxial deformation mode corresponds to an application of force along, e.g., the longitudinal axis of the specimen. For the purpose of explanation here, we denote this axis $x 1$. Then the specimen is free to expand or contract along axes $x 2$ and $x 3$. The equibiaxial deformation mode corresponds to equal stretches along the $\mathrm{x} 1$ and $\mathrm{x} 2$ axes, with unrestrained movement along the $\mathrm{x} 3$ axis, and planar deformation corresponds to a stretch along the $x 1$ axis, with no stretch allowed along the $x 2$ axis, and unrestrained movement along the $\times 3$ axis. Each of these modes is an example of a deformation in which the directions of principal strain do not change. Each mode can be written in terms of a single stretch, which allows the stress-strain relationship to be easily measured.

For an incompressible material, uniaxial compression is equivalent to equibiaxial tension, uniaxial tension is equivalent to equibiaxial compression, and planar tension is equivalent to planar compression (ABAQUS 1995a). For this analysis, experimental testing was performed in uniaxial tension and uniaxial compression, and is described in detail in Sections 3.3.1 and 3.3.2. It was felt necessary to include both tension and compression data. The exclusion of compression data may result in a strain energy function that does not capture much of the strain energy dependence on $I_{2}$. A planar test would have been done had we had the appropriate equipment, and may have captured dependence on the strain invariants that was missed by the uniaxial tests. In lieu of an actual planar test, we calculated the stress-strain response in planar tension using the Valanis-Landel function (c.f. Section 3.4). In order to determine the volumetric parameter $D_{i}$, which is needed to model compressibility, an experimental volumetric test was performed. The volumetric test and results are described in detail in Section 3.3.3. 


\subsection{Experiments Performed on Rubber for Finite Element Modeling}

\subsubsection{Rubber Uniaxial Tension Testing}

The uniaxial tension tests were carried to engineering tensile strains of 6.00 $\mathrm{m} / \mathrm{m}$, very near the ultimate elongation of the rubber, in order to ensure that the strain energy function would adequately represent the severe engineering tensile stresses and strains expected in the finite element analysis.

The bearing manufacturer supplied two vulcanized sheets of rubber for each of the four batches and the composite batch, which consisted of equal proportions of the four batches. As discussed in Section 2.1.2.1, the bearings were manufactured from three batches of rubber. A fourth batch was rejected because it was calendered (rolled) to the wrong thickness. Therefore, a sample from the composite batch is representative of each of the batches. Each sheet was prepared in accordance with ASTM D 3182-89.

The calendering of the rubber sheets may have a measurable influence on tensile properties since anisotropy or grain directionality is introduced in the calendering process. The calendered specimens are placed in a mold, and then compressed. We assume that the identification label is consistent with respect to the direction of rolling, i.e. if the label is parallel to the direction of rolling, then it is consistently parallel. The dumbbell specimens were cut both parallel and perpendicular to the identification label in order to test whether the tensile response was sensitive to direction. Figure 3.2 shows the sheets.

The dimensions of the dumbbell specimens are shown in Figure 3.3. These were cut using the Standard Die C (ASTM D 412-92), and provided a reduced section width of $6.35 \mathrm{~mm}(1 / 4 \mathrm{in})$. The thickness of each specimen was measured in accordance with ASTM D412-92, three measurements being made, one at the center and one at each end of the reduced section. The average of these figures was used as the thickness. The specimens were generally about $2.0 \mathrm{~mm}$ thick. The difference between the maximum and minimum thicknesses was less than $0.08 \mathrm{~mm}(0.003 \mathrm{in})$ for each specimen. Consequently, no specimens were discarded, in accordance with ASTM D412-92.

\subsubsection{Rubber Uniaxial Tension Testing Procedure}

Testing was performed on an Instron 1125 test machine using an Instron $222.4 \mathrm{~N}$ (50 lb) load cell. MTS TestWorks Software Version 3.05-9 was used to control the velocity of the load head. This software automatically records load and displacement, however, the recorded displacement is the grip separation. This displacement measurement is undesirable because it includes end effects in the dumbbell specimen. To improve the strain measurement, gage marks were placed in the reduced section, equidistant from the center and perpendicular to the longitudinal axis, and displacement readings were manually recorded using a caliper with a digital measurement display accurate to $0.01 \mathrm{~mm}$. After a displacement and corresponding force data pair were recorded, the caliper opening was increased close to the next desired displacement reading. In practice, displacement readings differed from sample to sample, since the important thing was to get reasonably spaced measurements with accurate force readings at those displacements, and not to obtain the readings at absolutely predetermined displacements. Setting the caliper at an exactly precise displacement was time consuming, especially for the tests done at faster strain rates. Consequently, 
the recorded displacements, hence strains, differed from one specimen to another over the entire strain range.

No mechanical conditioning of the specimens was done. Tests were performed at four strain rates, as shown in the Table 3.1. In the actual bearing tests, the compressive nominal strain rates varied from $0.000316 \mathrm{~m} / \mathrm{m} / \mathrm{s}$ to $0.000861 \mathrm{~m} / \mathrm{m} / \mathrm{s}$.

Table 3.1 - Strain rates for rubber tensile tests

\begin{tabular}{cc}
\hline Head Velocity $(\mathrm{mm} / \mathrm{min})$ & Strain Rate (per second) \\
\hline 2.282 & 0.000499 \\
10.000 & 0.002187 \\
25.400 & 0.005556 \\
200.000 & 0.043745 \\
\hline
\end{tabular}

\subsubsection{Rubber Uniaxial Tension Testing - Observations and Results}

Figure 3.4 shows the stress-strain plots of all tensile tests. There is no clear difference in response between specimens cut parallel to the identification label ("Hor" specimens) and those cut perpendicular to the identification label ("Vert" specimens), nor by strain rate. The specimen Batch1.Hor\#2 was chosen because the stress-strain curve represents about an average over all the curves. Averaging of all tests was not done, since this would have required curve fitting each test, since the stress values of each test were at different strains, as discussed in the preceding section.

\subsubsection{Rubber Uniaxial Compression Testing}

The B-Bearings were tested to a bearing engineering compressive stress of just under $100 \mathrm{MPa}$, so the uniaxial compressive tests were carried to a stress level exceeding $100 \mathrm{MPa}$ (actually, to approximately $125 \mathrm{MPa}$ ). At this stress level, the engineering compressive strain exceed $0.85 \mathrm{~m} / \mathrm{m}$. This ensured that the strain energy function would adequately represent the severe compressive stresses and strains expected in the finite element analysis.

The bearing manufacturer provided samples normally used for the ASTM Compression Set Test (ASTM D 395-89). These samples were labeled CompSet \#. A second set were samples designed to test the hardness of the rubber by use of the Shore A durometer (ASTM D 2240-91), and were labeled Duro \#. Figure 3.5 shows the dimensions and shape factors of each type of specimen.

\subsubsection{Rubber Uniaxial Compression Testing Procedure}

Compression testing was performed on the Instron 1125 using a $98.1 \mathrm{KN}$ $(10,000 \mathrm{~kg}-\mathrm{f})$ load cell. The vertical displacement was recorded electronically, using MTS TestWorks Software. In order to get accurate displacement readings, the machine compliance had to be removed from the displacement data. The machine compliance is 
the measured vertical displacement that is due solely to the flexibility of the testing machine. Compliance is significant in this test because the rubber samples resisted a high load. Compliance was found by compressing the platens together, without the specimen in place, using the same configuration as if a specimen were being tested. The resulting data are plotted as displacement versus load, and the displacement (compliance) is found as a function of load using a polynomial curvefit. The compliance is removed at each load value in the actual test data. At $109 \mathrm{MPa}$, the compliance accounts for $7.2 \%$ of the measured displacement for the Compression Set specimen and $14.9 \%$ for the Durometer specimen.

The uniaxial compression test must simulate homogeneous compression. In homogeneous deformation, straight lines before deformation remain straight after deformation (Eringen, 1989). In the compression test performed, this requires the loaded ends to slide freely along the loaded surface, so there is no bowing of the unloaded surface. Compression with the top and bottom surfaces bonded to the platens is the other extreme - there is no sliding along the loaded surface. The stress-strain response of a bonded specimen is a function of the shape factor. The larger the shape factor, the stiffer is the response. Figure 3.6 shows the difference between homogeneous and bonded compression.

Attaining perfect homogeneous compression requires a frictionless contact between the platen and sample surface. In order to simulate frictionless conditions as closely as possible, Apiezon grease was applied to the loaded surfaces prior to the test.

The load head platens had concentric grooves in them, making it difficult to simulate a frictionless surface. Smooth high strength steel plates were used between the platen and sample to reduce friction. The upper plate could not be attached to the upper load head. This made balancing of the smooth platen difficult, especially since the specimen was greased, and necessitated a slight preload to keep the upper platen from sliding to one side or the other.

\subsubsection{Rubber Uniaxial Compression Testing - Observations and Results}

Bowing of the unloaded surface was very difficult to determine visually because grease squeezing out from the edges of the loaded surfaces obscured the view. Figure 3.7 shows the stress-strain plots of CompSet 3 and CompSet 4 prior to removal of the machine compliance. These two virtually identical results show repeatability in the test, indicating more reliability in the results.

Figure 3.8 shows the stress-strain curves of CompSet 4 and Duro 1 before and after removal of the machine compliance. The "CompSet" specimen refers to a specimen that was originally made for the ASTM compression set test (ASTM D 39589), and the "Duro" specimen refers to a specimen originally made for the ASTM durometer (hardness) test (ASTM D 2240-91). The curves are virtually identical after removal of machine compliance, and indicates a good approximation to homogeneous compression, since the shape factors are different. The Duro 1 plot is shifted more by removal of the compliance because it is a thinner specimen than CompSet 4 , so each compliance value is a higher vertical strain. CompSet 4 was chosen for input into the finite element program. 


\subsubsection{Volumetric Testing of Rubber}

The volumetric testing was performed on the Instron 1125 using a $98.1 \mathrm{KN}$ (10,000 kg-f ) load cell, the same load cell used for compression testing. Figure 3.9 shows a diagram of the device that was manufactured for volumetric testing. The cylindrical hole was made at the specified diameter in order to ensure a snug fit with the available samples. The Compression Set samples CompSet 5, CompSet 6, and CompSet 7 were tested in this apparatus.

The tight tolerances were necessary because of the extreme importance of providing no room for the rubber to extrude during the test. The piston slid freely through the hole when the bottom plate was removed, but with the bottom plate attached, entrapped air could not move around the piston quickly, thereby retarding its movement. This was not a problem, since the tests were run at low head speeds.

As with the compression testing, compliance tests were used to remove the effects of the inherent flexibility of the machine. This flexibility was removed in the same fashion as described above for the compression testing (c.f. Section 3.3.2.1).

Load was applied to the piston, and the load and corresponding vertical displacement of the piston were measured electronically. Figure 3.10 shows the load vs extension for each of the three samples tested prior to removal of the machine compliance. The slopes are virtually identical. Each specimen has a very low initial stiffness, which appears as a horizontal line. This initial low stiffness is probably due to the rubber squeezing into small voids because the specimens weren't perfectly cylindrical.

After removal of the machine compliance, the raw data were converted into Pressure Stress and Volume Ratio. Figure 3.11 shows the plot for CompSet 7 . Note that removal of the machine compliance does not account for the initial low stiffness in the response.

\subsubsection{Rubber Model used in Finite Element Analysis (based on Experimental Data)}

A particular set of stress-strain points from the tensile test Batch1.Hor \#2 and the compression test CompSet 4 were chosen for input into the finite element program. This set of uniaxial engineering stress-strain data points is referred to as Rubber Model 1 (RM1), and is shown in Figure 3.12. As discussed in Section 3.2, this data is used in an ABAQUS analysis to determine the coefficients for the deviatoric portion of the strain energy functions, specifically the $C_{i j}$ for the Polynomial Strain Energy Function, and the $\mu_{i}$ and $\alpha_{i}$ for the Ogden Strain Energy Function.

To determine the coefficients $D_{i}$ used in determining the form of the volumetric portion of both strain energy functions, data from test CompSet 7 is used, and is referred to subsequently as CS7. Figure 3.13 shows the actual volumetric data used in the finite element model. The low initial stiffness has been removed from the data. As discussed in Section 3.3.3, the low initial stiffness is probably due to rubber squeezing into small voids since the specimens weren't perfectly cylindrical. 


\subsubsection{Measurement Accuracy and Variability and Effect on Bearing Response}

The accuracy of the Instron 1125 test machine is established through a calibration using known dead loads, and was calibrated according to ASTM E4, which requires that the machine reading be within $\pm 1 \%$ of the true reading. The machine has met these requirements. This is equivalent to a relative expanded uncertainty $(\mathrm{k}=2)$ of $1 \%$, and is small compared to specimen variability for the rubber uniaxial testing.

Rubber uniaxial tensile testing was performed on fourteen (14) dumbbell specimens. The variation about the mean of these tests was approximately $\pm 10 \%$, so the variability can be attributed to factors outside the resolution of the testing machine. Sources of variation include (1) differences in the properties of the rubber and (2) measurement error. Displacements were recorded manually using a hand-held caliper, and force readings were manually taken at the time of each displacement reading. Measurement error in the cross-sectional area can also affect the results. Based on this limited testing sample and manual testing methodology, it is difficult to say how much of the variability is due strictly to differences in the properties of the rubber, but it is likely that the largest portion of the variability is due to the manual measurement process.

Rubber uniaxial compression testing was performed on cylindrical bullets of different shape factors, and the data was recorded electronically, as discussed in Section 3.3.2 above. For the two tests reported and shown graphically in Figure 3.8, the variation of the test values about the mean of the two tests was approximately $\pm 2.8 \%$.

Volumetric testing was performed on cylindrical bullets, and the data was recorded electronically, as discussed in Section 3.3.3 above. For the three tests reported and shown graphically in Figure 3.10, the slopes of each graph, which correspond to the bulk modulus, differ by only $\pm 0.86 \%$ from the mean value. This difference cannot be attributed to any specific errors in testing or differences in the bulk modulus from sample to sample because these values lie inside the resolution of the testing machine.

The finite element analysis of the bearings, discussed in detail in Section 6 , shows that the hydrostatic stresses in the rubber are two orders of magnitude larger than the deviatoric stresses. The uniaxial tests serve to define the deviatoric strain energy, so any variation in the uniaxial testing results would have a negligible affect on the bearing compressive stress-strain results. The hydrostatic stresses play the major role here, but the variation in the three tests is so small that we cannot attribute differences in the bearing responses to differences in the bulk modulus of the rubber from sample to sample.

\subsection{Extension of Rubber Experimental Stress-Strain Data to Equibiaxial and Planar Deformation Modes using the Valanis-Landel Function}

Rubber testing was performed only in the uniaxial tensile and compressive modes. A mode of deformation independent of either uniaxial tension or uniaxial compression is the planar deformation mode. No planar deformation tests were done, since a test apparatus for this mode of deformation was not available. Planar deformation is referred to as a pure shear. A simple shear is equivalent to a pure shear plus a rotation. Since shearing stresses are present at the rubber/steel interface, accurate determination of these stresses is important. Since it was not clear apriori that uniaxial 
stress-strain curves would result in a strain energy function that would properly characterize this mode of deformation, we used the Valanis-Landel function to generate stress data for planar deformation as if we had performed the tests ourselves. Also, equibiaxial tension, which is equivalent to uniaxial compression for an incompressible material, was also calculated from the Valanis-Landel function.

The inclusion of planar tension data might result in a strain energy function which better describes the behavior of the rubber in all deformation modes. This section describes in detail how the Valanis-Landel function is found and used to generate these other data sets. In Section 3.5, the reasonableness of the planar and equibiaxial stress-strain data is evaluated. In Section 4, finite element runs are presented to see how well the ABAQUS code predicts the experimental and ValanisLandel generated data.

For an elastic, isotropic, and incompressible material, stress differences on a unit cube of material can be written as

$$
\begin{aligned}
& t_{1}-t_{2}=\lambda_{1} \frac{\partial U}{\partial \lambda_{1}}-\lambda_{2} \frac{\partial U}{\partial \lambda_{2}} \\
& t_{2}-t_{3}=\lambda_{2} \frac{\partial U}{\partial \lambda_{2}}-\lambda_{3} \frac{\partial U}{\partial \lambda_{3}} \\
& t_{3}-t_{1}=\lambda_{3} \frac{\partial U}{\partial \lambda_{3}}-\lambda_{1} \frac{\partial U}{\partial \lambda_{1}}
\end{aligned}
$$

where directions 1,2 , and 3 correspond to a right handed orthogonal coordinate system, $t_{i}$ is the true stress on the face perpendicular to the $i$-direction, and $\lambda_{i}$ is the stretch ratio, defined as the ratio of the length of a line in the deformed configuration to the length of that line in the undeformed configuration which initially points in the $i$ direction.

As noted in Section 3.2, Valanis and Landel (1967) postulate that the strain energy function for rubber can be written as a separable function of the stretch ratios, as follows:

$$
U=u\left(\lambda_{1}\right)+u\left(\lambda_{2}\right)+u\left(\lambda_{3}\right)
$$

where $u(\cdot)$ is termed the Valanis-Landel function. Taking the derivative with respect to the stretch, we get

$$
\frac{\partial U}{\partial \lambda_{i}}=\frac{d\left[u\left(\lambda_{i}\right)\right]}{d \lambda_{i}} \text { or } u^{\prime}\left(\lambda_{i}\right)
$$

and Equation (3.4) can be rewritten as

$$
\begin{aligned}
& t_{1}-t_{2}=\lambda_{1} u^{\prime}\left(\lambda_{1}\right)-\lambda_{2} u^{\prime}\left(\lambda_{2}\right) \\
& t_{2}-t_{3}=\lambda_{2} u^{\prime}\left(\lambda_{2}\right)-\lambda_{3} u^{\prime}\left(\lambda_{3}\right)
\end{aligned}
$$




$$
t_{3}-t_{1}=\lambda_{3} u^{\prime}\left(\lambda_{3}\right)-\lambda_{1} u^{\prime}\left(\lambda_{1}\right)
$$

For a planar tension test with loading in the 1 direction and no deformation in the 2 direction, $\lambda_{2}=1$ and $t_{3}=0$. Applying the incompressibility constraint $\lambda_{1} \lambda_{2} \lambda_{3}=1$, we get $\lambda_{3}=\frac{1}{\lambda_{1}}$. Thus, Equation (3.7c) becomes

$$
t_{1}=\lambda_{1} u^{\prime}\left(\lambda_{1}\right)-\frac{1}{\lambda_{1}} u^{\prime}\left(\frac{1}{\lambda_{1}}\right)
$$

To convert Equation (3.8) to engineering stress, note that $t_{i}=\frac{\text { force }}{\text { original area }} * \frac{\text { original area }}{\text { current area }}=\sigma_{i} * \frac{\text { originalarea }}{\text { current area }}$, where $\sigma_{i}=$ engineering stress. In terms of stretch ratios, $t_{i}=\sigma_{i} * \frac{1}{\lambda, \lambda_{k}}=\sigma_{i} \lambda_{i}$, and the engineering stress in the planar mode, in terms of the derivative of the Valanis-Landel function , is

$$
\sigma_{1}=u^{\prime}\left(\lambda_{1}\right)-\frac{1}{\lambda_{1}^{2}} u^{\prime}\left(\frac{1}{\lambda_{1}}\right)
$$

For an equibiaxial tension test with loading in the 1 and 2 directions, $t_{3}=0$ and $t_{1}=t_{2}$. In this case, Equation (3.7a) is identically satisfied, and Equations (3.7b) and (3.7c) give the result

$$
t_{2}=\lambda_{2} u^{\prime}\left(\lambda_{2}\right)-\lambda_{3} u^{\prime}\left(\lambda_{3}\right)
$$

Applying the incompressibility constraint, and noting that $\lambda_{2}=\lambda_{1}$, we get

$$
t_{2}=\lambda_{2} u^{\prime}\left(\lambda_{2}\right)-\frac{1}{\lambda_{2}^{2}} u^{\prime}\left(\frac{1}{\lambda_{2}^{2}}\right)
$$

Therefore, engineering stress in the equibiaxial mode, in terms of the derivative of the Valanis-Landel function, is

$$
\sigma_{2}=u^{\prime}\left(\lambda_{2}\right)-\frac{1}{\lambda_{2}^{3}} u^{\prime}\left(\frac{1}{\lambda_{2}^{2}}\right)
$$

Once the derivative of the Valanis-Landel function is determined, then the stress-strain curves for equibiaxial and planar tension can be computed. This function can be found from the existing uniaxial test data. For a uniaxial test with loading in the 1-direction, $\lambda_{2}=\lambda_{3}$. Applying the incompressibility constraint $\lambda_{2}=\lambda_{3}=\frac{1}{\sqrt{\lambda_{1}}}$, and noting that $t_{2}=0$, Equation (3.7a) becomes

$$
t_{1}=\lambda_{1} u^{\prime}\left(\lambda_{1}\right)-\lambda_{1}^{-1 / 2} u^{\prime}\left(\lambda_{1}^{-1 / 2}\right)
$$


This form, however, does not lend itself to an easy identification of the derivative of the Valanis-Landel function. A solution to this problem was found by Kearsley and Zappas (1980), as follows:

Rewriting Equation (3.13) with the argument $\lambda_{1}^{-1 / 2}$ yields

$$
t_{1}\left(\lambda_{1}^{-1 / 2}\right)=\lambda_{1}^{-1 / 2} u^{\prime}\left(\lambda_{1}^{-1 / 2}\right)-\left(\lambda_{1}^{-1 / 2}\right)^{-1 / 2} u^{\prime}\left(\left(\lambda_{1}^{-1 / 2}\right)^{-1 / 2}\right)=\lambda_{1}^{-1 / 2} u^{\prime}\left(\lambda_{1}^{-1 / 2}\right)-\lambda_{1}^{4 / 4} u^{\prime}\left(\lambda_{1}^{1 / 4}\right)
$$

Adding Equations (3.13) and (3.14) together, one gets

$$
t_{1}\left(\lambda_{1}\right)+t_{1}\left(\lambda_{1}^{-1 / 2}\right)=\lambda_{1} u^{\prime}\left(\lambda_{1}\right)-\lambda_{1}^{1 / 4} u^{\prime}\left(\lambda_{1}^{1 / 4}\right)
$$

Note that the two middle terms cancel out. Continuing this process, we get two additional equations,

$$
\begin{gathered}
t_{1}\left(\lambda_{1}^{1 / 4}\right)=\lambda_{1}^{1 / 4} u^{\prime}\left(\lambda_{1}^{1 / 4}\right)-\lambda_{1}^{-1 / 8} u^{\prime}\left(\lambda_{1}^{-1 / 8}\right), \text { and } \\
t_{1}\left(\lambda_{1}^{-1 / 8}\right)=\lambda_{1}^{-1 / 8} u^{\prime}\left(\lambda_{1}^{-1 / 8}\right)-\lambda_{1}^{416} u^{\prime}\left(\lambda_{1}^{116}\right)
\end{gathered}
$$

and adding these two equations to Equation (3.15), we get

$$
t_{1}\left(\lambda_{1}\right)+t_{1}\left(\lambda_{1}^{-1 / 2}\right)+t_{1}\left(\lambda_{1}^{1 / 4}\right)+t_{1}\left(\lambda_{1}^{-1 / 8}\right)=\lambda_{1} u^{\prime}\left(\lambda_{1}\right)-\lambda_{1}^{4 / 16} u^{\prime}\left(\lambda_{1}^{1 / 16}\right)
$$

Grouping the first and third terms, and the second and fourth terms, we can rewrite Equation (3.18) as $\sum_{k=0}^{1} t_{1}\left(\lambda_{1}^{(1 / 4)^{k}}\right)+t_{1}\left(\lambda_{1}^{-(/ 2)(1 / 4)^{k}}\right)=\lambda_{1} u^{\prime}\left(\lambda_{1}\right)-\lambda_{1}^{1 / 16} u^{\prime}\left(\lambda_{1}^{1 / 16}\right)$. We can continue this process and obtain the series

$$
\sum_{k=0}^{n-1}\left[t_{1}\left(\lambda_{1}^{(1 / 4)^{k}}\right)+t_{1}\left(\lambda_{1}^{-(1 / 2)(1 / 4)^{k}}\right)\right]=\lambda_{1} u^{\prime}\left(\lambda_{1}\right)-\lambda_{1}^{(1 / 4)^{k}} u^{\prime}\left(\lambda_{1}^{(1 / 4)^{n}}\right)
$$

Taking the limit of this expression as $n \rightarrow \infty$, Equation (3.19) becomes

$$
\lim _{n \rightarrow \infty} \sum_{k=0}^{n-1}\left[t_{1}\left(\lambda_{1}^{(1 / 4)^{k}}\right)+t_{1}\left(\lambda_{1}^{-(1 / 2)(1 / 4)^{k}}\right)\right]=\lambda_{1} u^{\prime}\left(\lambda_{1}\right)-u^{\prime}(1)
$$

Since $\lambda=1$ corresponds to no deformation, $u^{\prime}(1)=0$.

The right hand side of Equation (3.20) $\left(\lambda u^{\prime}(\lambda)\right)$ is an infinite sum of the true stresses at the stretches specified in the bracketed expression, but this sum converges rapidly. Note that the first and second terms are on opposite sides of the undeformed state $(\lambda=1)$, so that both extension and compression data are required, and the series terms oscillate around the undeformed state and rapidly approach it.

In calculating Equation (3.20), the same uniaxial data set was used as for RM1; tension data from Batch1.Hor\#2 and compression data from CompSet 4. Every data point in this set was used. The experimental uniaxial data is converted to true stressstrain data by multiplying each stress by its corresponding stretch. Figure 3.14 shows the true stress-strain plot. To aid in the calculations, it was necessary to obtain a functional representation of the uniaxial stress-strain curve. Therefore, the curve was fitted with polynomial functions found from a least-square curvefitting procedure. Due 
to the sharp curvature, it was not possible to adequately fit the curve with one polynomial function over its entire domain. Instead, the domain was broken up into segments, each of which was curvefitted separately. Once completed, a computer program was written to find the values $u^{\prime}(\lambda)$ at desired stretch values.

Figure 3.15 shows the polynomial curvefit over a particular range of stretch values. A fifth order polynomial was used over this range. Over some other ranges, a ninth order polynomial was used. Figure 3.16 is a plot of $u^{\prime}(\lambda)$. A listing of the program used to find these values is shown in Appendix B. I

Table 3.2 is the output from a particular run of the program used to calculate $u^{\prime}(\lambda)$ and shows that convergence within 0.0001 occurs in less than 11 iterations.

Table 3.2 - Calculated values of the derivative of the Valanis-Landel Function $u^{\prime}(\lambda)$ Tolerance $=0.000100$

$\begin{array}{lllllr}\text { Max } & \text { \#Iters } & \text { Stretch } & \text { Sum[i] } & \text { Sum[i-1] } & \text { Difference } \\ 100 & 10 & 0.15 & -67.7246 & -67.7246 & 0.0000 \\ 100 & 9 & 0.35 & -0.3872 & -0.3871 & -0.0001 \\ 100 & 8 & 0.55 & 0.2652 & 0.2653 & -0.0001 \\ 100 & 8 & 0.75 & 0.1148 & 0.1148 & -0.0000 \\ 100 & 6 & 0.95 & 0.0048 & 0.0049 & -0.0001 \\ 100 & 7 & 1.15 & 0.7627 & 0.7627 & 0.0001 \\ 100 & 8 & 1.35 & 1.0948 & 1.0948 & 0.0000 \\ 100 & 8 & 1.55 & 1.3827 & 1.3826 & 0.0000 \\ 100 & 8 & 1.75 & 1.6109 & 1.6109 & 0.0000 \\ 100 & 8 & 1.95 & 1.9006 & 1.9005 & 0.0000 \\ 100 & 8 & 2.15 & 2.2284 & 2.2284 & 0.0000 \\ 100 & 8 & 2.35 & 2.5715 & 2.5714 & 0.0000 \\ 100 & 8 & 2.55 & 2.9837 & 2.9837 & 0.0000 \\ 100 & 8 & 2.75 & 3.4145 & 3.4145 & 0.0000 \\ 100 & 8 & 2.95 & 3.8874 & 3.8874 & 0.0000 \\ 100 & 8 & 3.15 & 4.3750 & 4.3749 & 0.0000 \\ 100 & 8 & 3.35 & 4.8754 & 4.8754 & 0.0000 \\ 100 & 8 & 3.55 & 5.3961 & 5.3961 & 0.0000 \\ 100 & 8 & 3.75 & 5.9439 & 5.9439 & 0.0000 \\ 100 & 8 & 3.95 & 6.5195 & 6.5195 & 0.0000 \\ 100 & 8 & 4.15 & 7.1172 & 7.1171 & 0.0000 \\ 100 & 8 & 4.35 & 7.7281 & 7.7281 & 0.0000 \\ 100 & 8 & 4.55 & 8.3451 & 8.3450 & 0.0000 \\ 100 & 8 & 4.75 & 8.9669 & 8.9668 & 0.0000 \\ 100 & 8 & 4.95 & 9.5974 & 9.5974 & 0.0000 \\ 100 & 8 & 5.15 & 10.2452 & 10.2452 & 0.0000 \\ 100 & 8 & 5.35 & 10.9185 & 10.9185 & 0.0000 \\ 100 & 8 & 5.55 & 11.6196 & 11.6196 & 0.0000 \\ 100 & 8 & 5.75 & 12.3393 & 12.3393 & 0.0000 \\ 100 & 8 & 5.95 & 13.0581 & 13.0581 & 0.0000 \\ 100 & 8 & 6.15 & 13.7550 & 13.7549 & 0.0000 \\ 100 & 8 & 6.35 & 14.4305 & 14.4305 & 0.0000\end{array}$




\subsection{Assessment of the Valanis-Landel Function}

Equation (3.13) gives the true stress for a uniaxial test. Converting true stress to engineering stress, we get

$$
\sigma=u^{\prime}(\lambda)-\lambda^{-3 / 2} u^{\prime}\left(\lambda^{-1 / 2}\right) .
$$

Figures 3.17 and 3.18 show the comparison between the experimental data and the engineering stresses generated by Equation (3.21) for uniaxial tension and compression, respectively. The results are in agreement with the experimental data, giving us confidence in the evaluation of the derivative of the Valanis-Landel function.

The stress-strain data for equibiaxial tension were generated from Equation (3.12). Since uniaxial compression and equibiaxial tension are equivalent for an incompressible material, we can transform the experimental uniaxial compression data to equibiaxial tension and compare the results to the equibiaxial stresses predicted by the Valanis-Landel function. Figure 3.19 shows the equivalence between uniaxial compression and equibiaxial tension (ABAQUS 1995a). The stresses in Figure 3.19 are true (Cauchy) stresses, and the superposition of a hydrostatic tensile stress - $t$ on the uniaxial compressive stress $t$ results in an equibiaxial tensile stress $-t$, without any change in deformation.

With reference to Figure 3.19, the incompressibility assumption gives us, in terms of the stretches, the equation $\lambda_{x} \lambda_{y} \lambda_{z}=1$. Using this relationship, the stretch in the coordinate directions $x$ and $y$ is $\lambda=\sqrt{\frac{1}{\lambda_{2}}}$, and the equibiaxial engineering strain is

$$
e=\lambda-1=\sqrt{\frac{1}{\lambda_{z}}}-1
$$

The true uniaxial compressive stress $t$, in terms of the engineering uniaxial compressive stress $\sigma_{z}$, is

$$
t=\frac{\sigma_{z}}{\lambda_{x} \lambda_{y}}
$$

and the engineering equibiaxial stress, in terms of the true stress, is

$$
\sigma_{x}=t \lambda_{y} \lambda_{z}
$$

Noting that $\sigma_{x}=\sigma_{y}$, and denoting this stress as $\sigma_{\text {equibiaxial }}$, by combining Equations (3.22), (3.23), and (3.24),

$$
\sigma_{\text {equibiaxial }}=\sigma_{z} \lambda_{z}^{3 / 2}
$$

Equation (3.25) represents the equibiaxial stress-strain response that is based on the assumptions that the rubber is isotropic and incompressible. Figure 3.20 shows 
excellent agreement between the equibiaxial stresses calculated from Equation (3.25) and the stresses found from the Valanis-Landel function.

The stress-strain data for planar tension were generated from Equation (3.9). Figure 3.21 is a comparison between the planar tensile stresses and the experimental uniaxial tensile stresses, and it is seen that the planar stresses are at least as large as the uniaxial stresses. The relative values are consistent with experimental data reported by Treloar (1943), as shown in Figure 3.22. Furthermore, the force required to deform a specimen in planar tension should be at least as large as the force required in uniaxial tension because in the planar deformation mode, the specimen is fixed along an axis perpendicular to the direction of loading, whereas in the uniaxial mode, the specimen is free to move in this same direction.

\subsection{Rubber Models used in Finite Element Analysis (based on Experimental and Valanis-Landel Generated Data)}

Figure 3.23 shows the stress-strain curve for planar tension, as found from the Valanis-Landel function, together with the experimental uniaxial tensile and compressive stress-strain curve (RM1). Each of these curves represents an independent mode of deformation. Collectively, this stress-strain data set is termed the VL1 rubber model.

A third rubber model, termed VL2, is shown in Figure 3.24. This model differs from VL1 by substituting the equibiaxial data generated from the ValanisLandel function for the experimental uniaxial compression data.

Stresses for a stretch greater than 2.64 (strain of 1.64) for equibiaxial tension cannot be found because the lowest stretch value in the uniaxial tests is 0.14389 . Higher stretches exceed the limits of the underlying data set, i.e. from the second term of Equation (3.12); $u^{\prime}\left(\frac{1}{\lambda^{2}}\right)=u^{\prime}\left(\frac{1}{2.64^{2}}\right)=u^{\prime}(0.143)$.

Each rubber model, VL1 and VL2, is used by ABAQUS to determine the coefficients for the deviatoric portion of the strain energy functions, specifically the $C_{i j}$ coefficients for the Polynomial Strain Energy Function, and the $\mu_{i}$ and $\alpha_{i}$ coefficients for the Ogden Strain Energy Function.

In Section 4.1, finite element runs are presented to show how well the finite element program predicts the experimental and Valanis-Landel generated stress-strain curves, using the deviatoric rubber models RM1, VL1, and VL2, and to show how well the finite element program predicts the experimental volumetric compression test through use of volumetric rubber model CS7. 


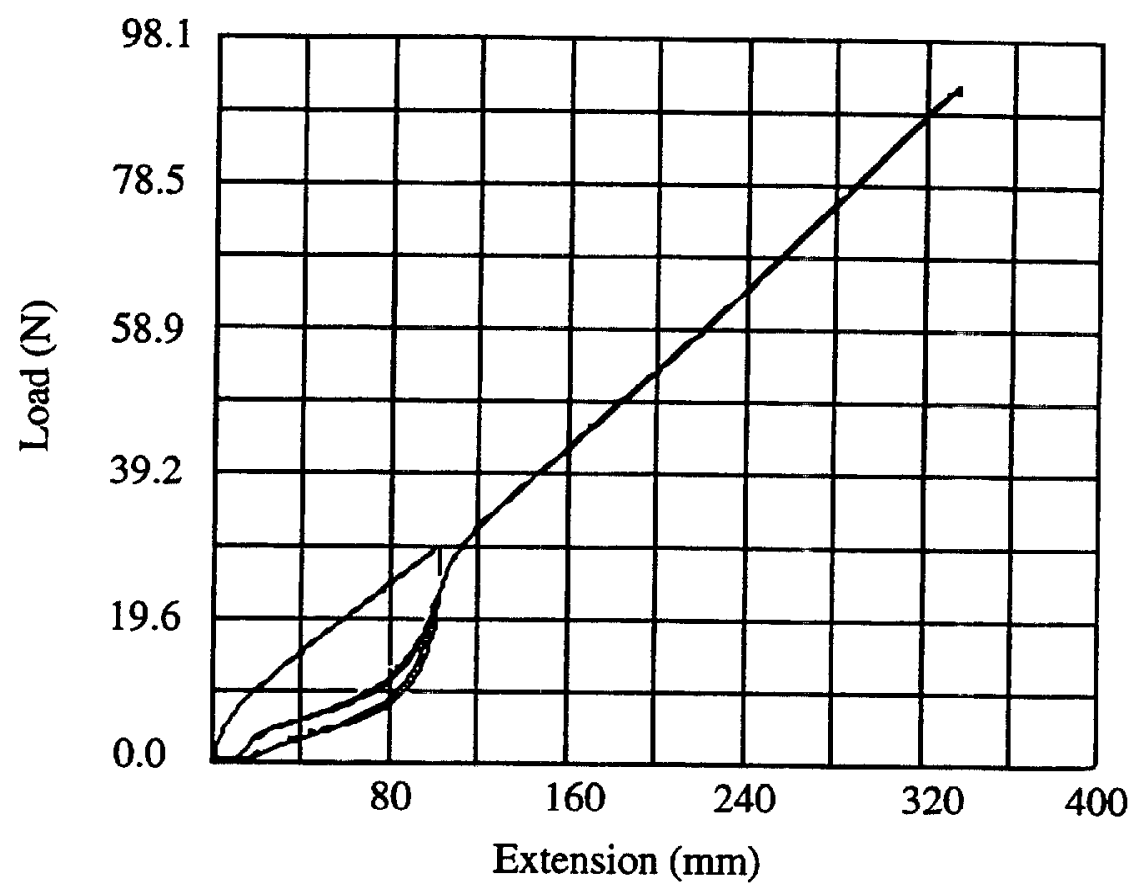

Figure 3.1 - Effect of mechanical conditioning on the stress-strain response of rubber

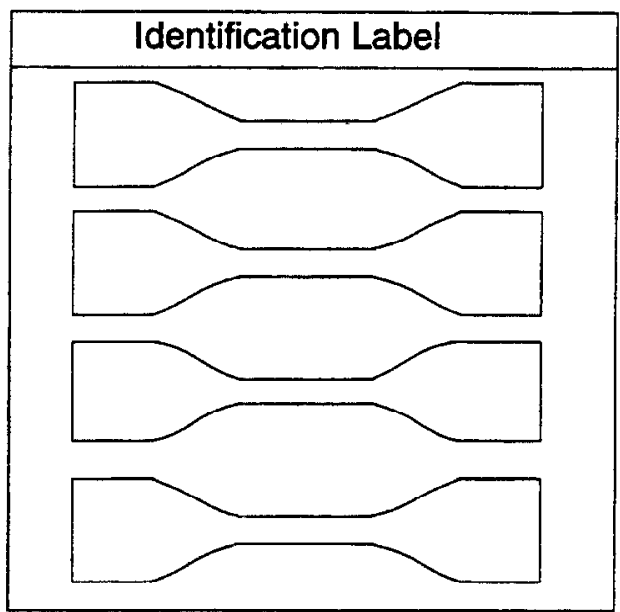

Specimens labelled Batch\#.Hor



Specimens labelled Batch\#.Vert

Figure 3.2 - Rubber sheets from which tensile specimens were cut 


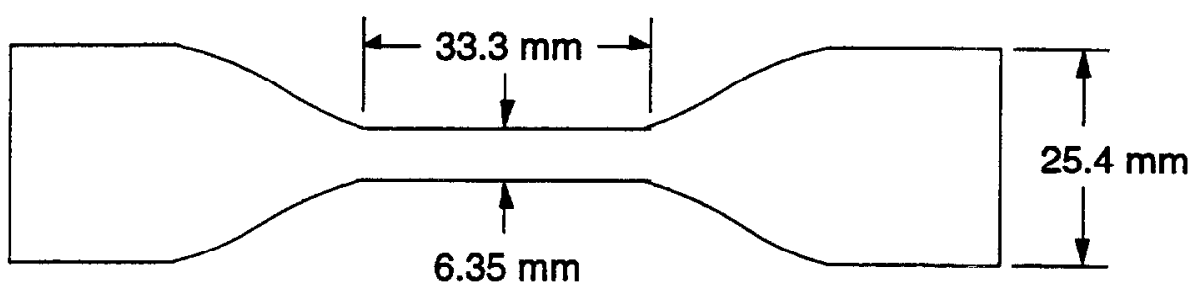

Figure 3.3 - Dimensions of rubber tensile specimens

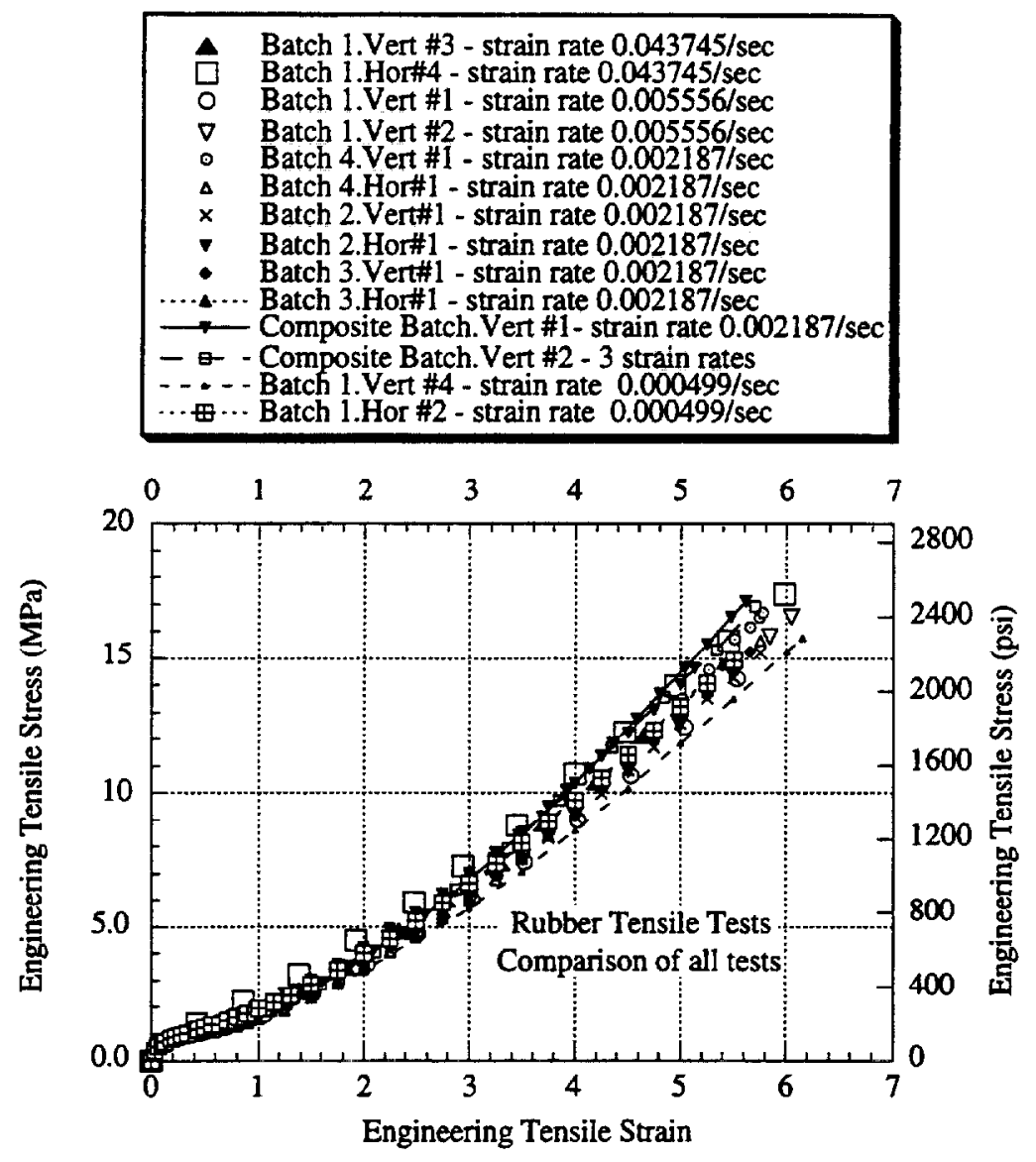

Figure 3.4 - Stress-strain curves for all rubber tensile tests 


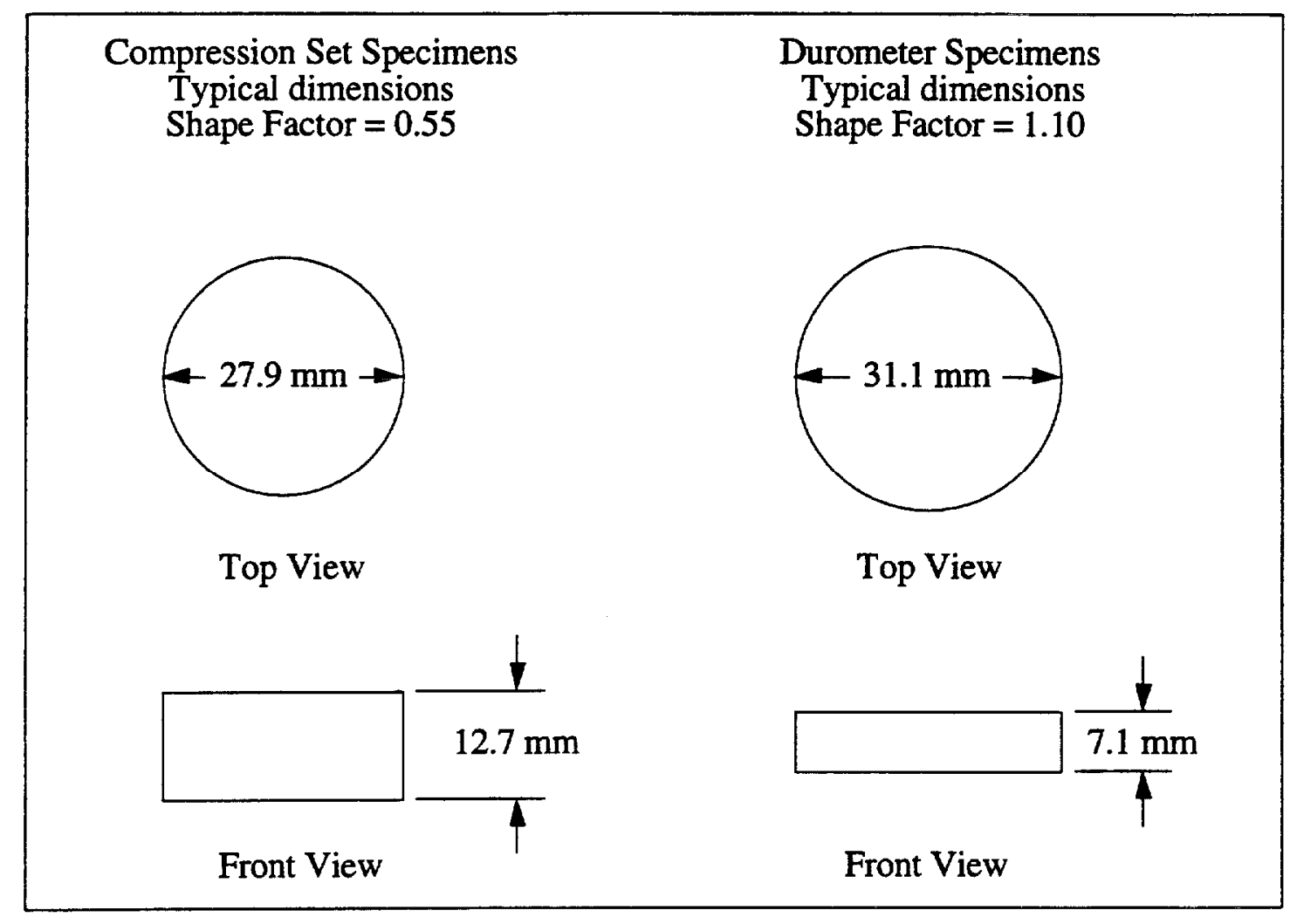

Figure 3.5 - Dimensions of rubber specimens used for compression testing 

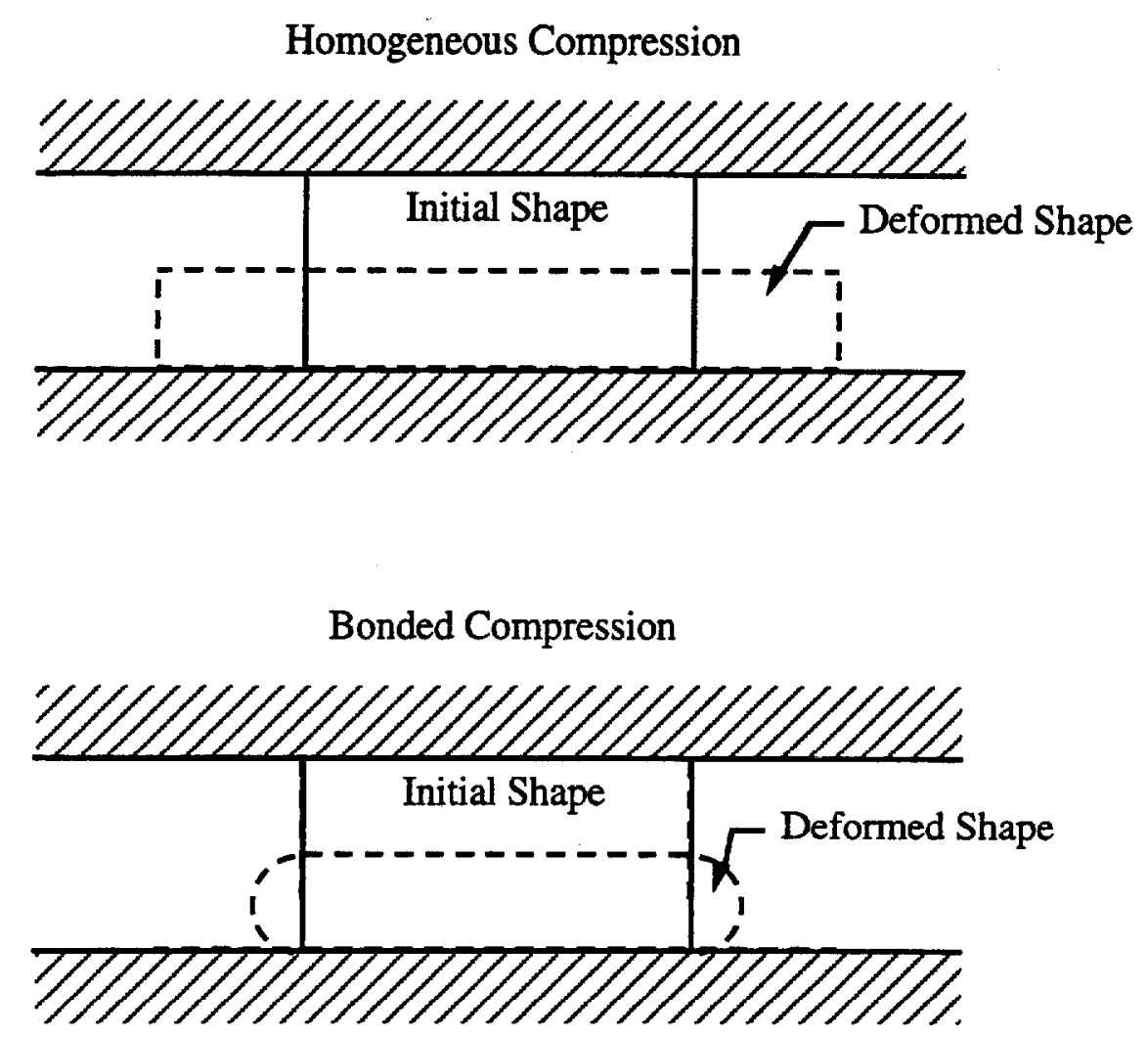

Figure 3.6 - Homogeneous vs bonded compression 


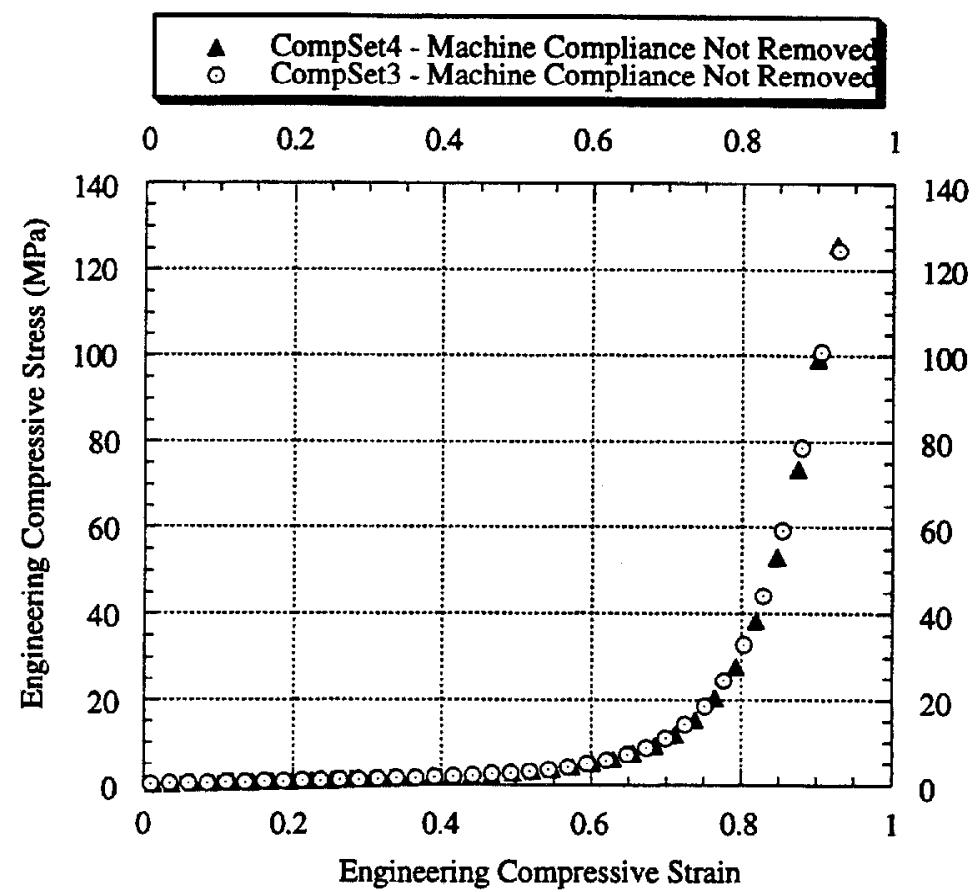

Figure 3.7 - Comparison of compressive response of two compression set specimens

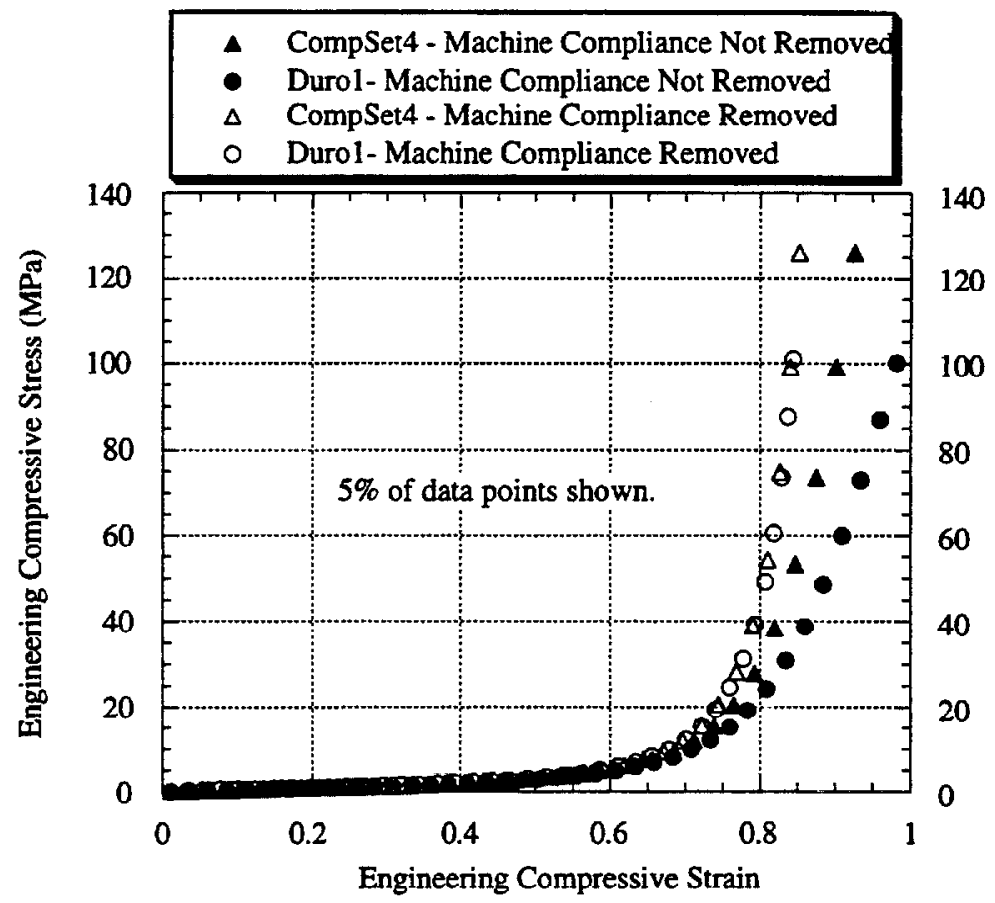

Figure 3.8 - Comparison of compressive response of compression set and durometer specimens 




Applied Load

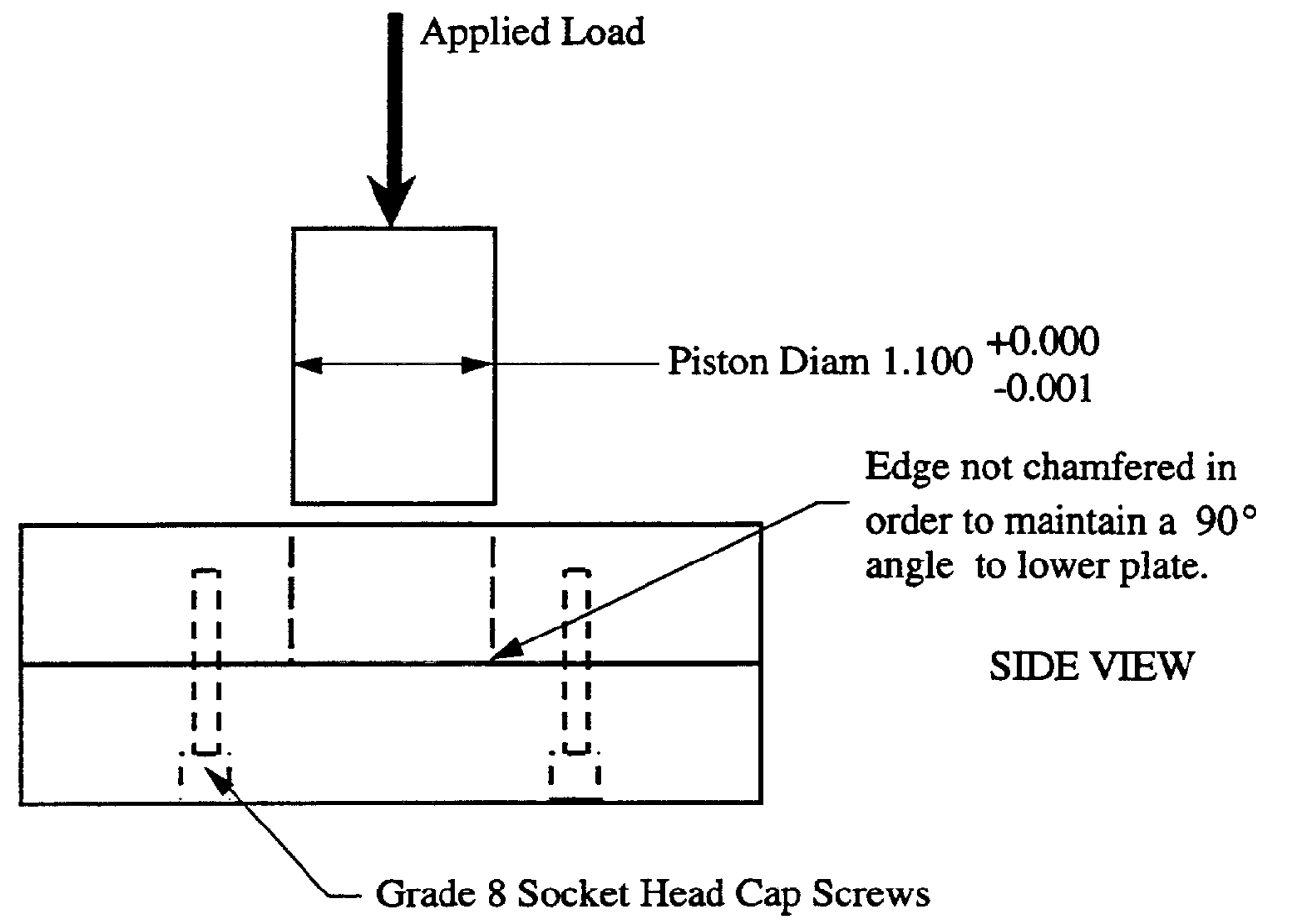

Figure 3.9 - Device used for volumetric testing 


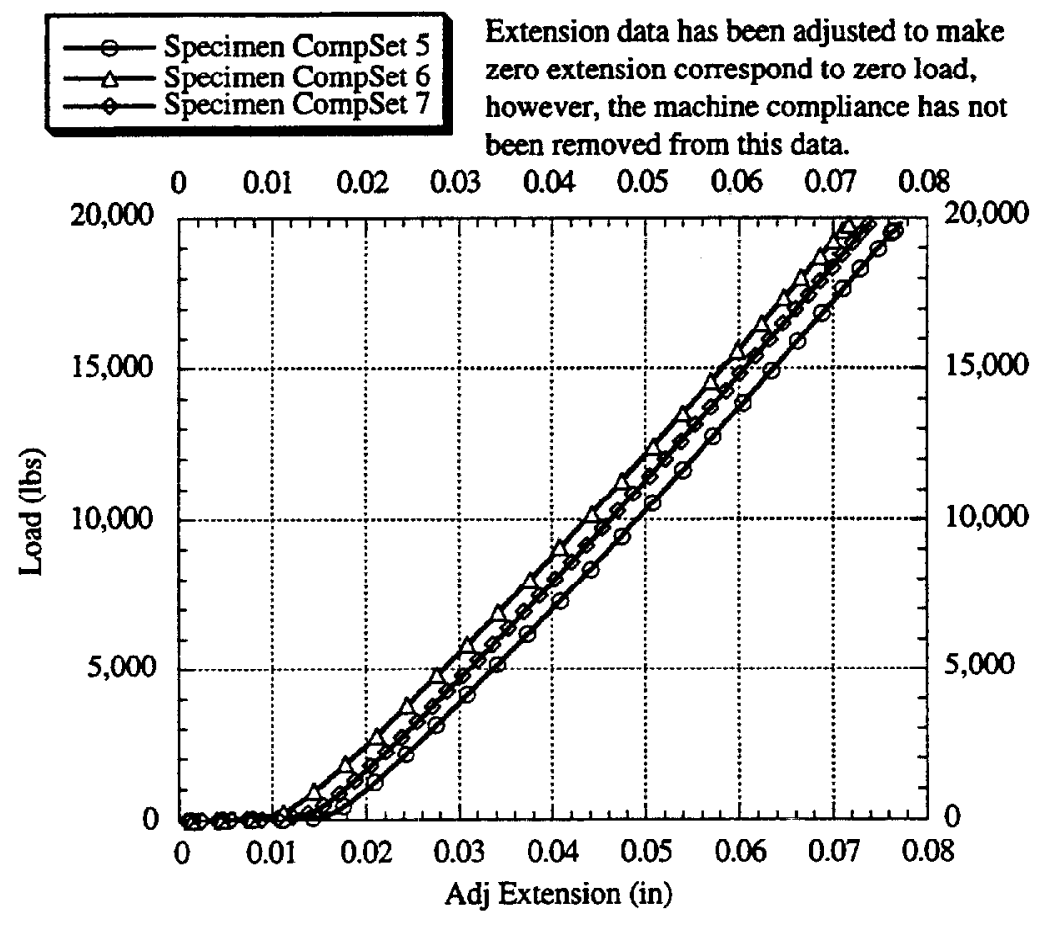

Figure 3.10 - Comparison of volumetric compression tests

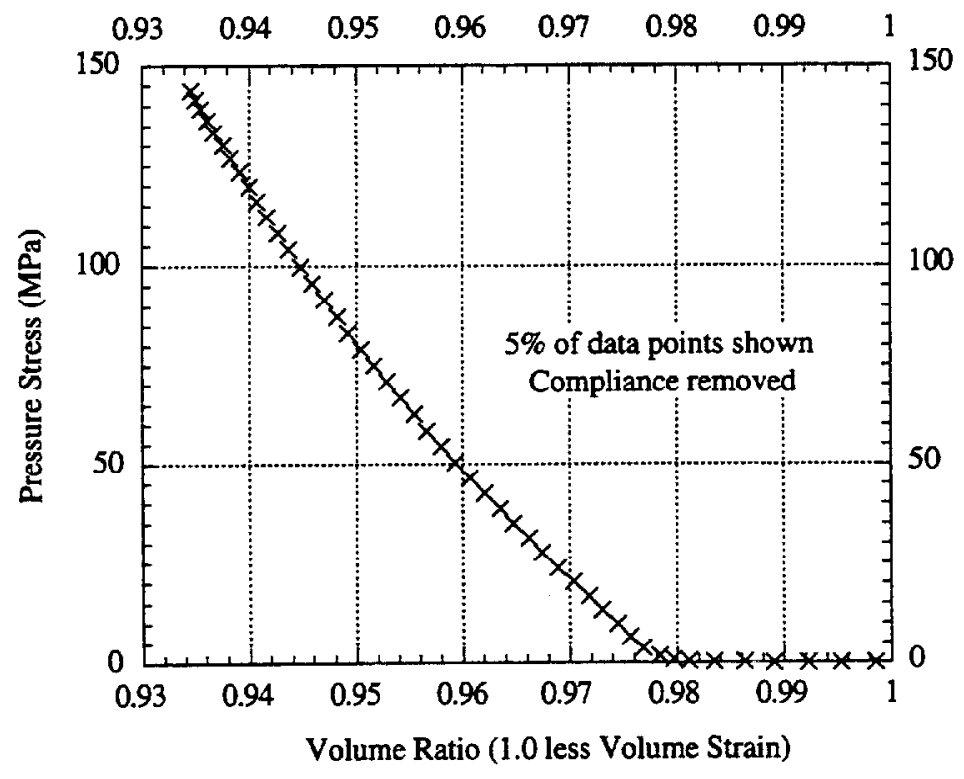

Figure 3.11 - CompSet 7 (CS7) volumetric compression test 


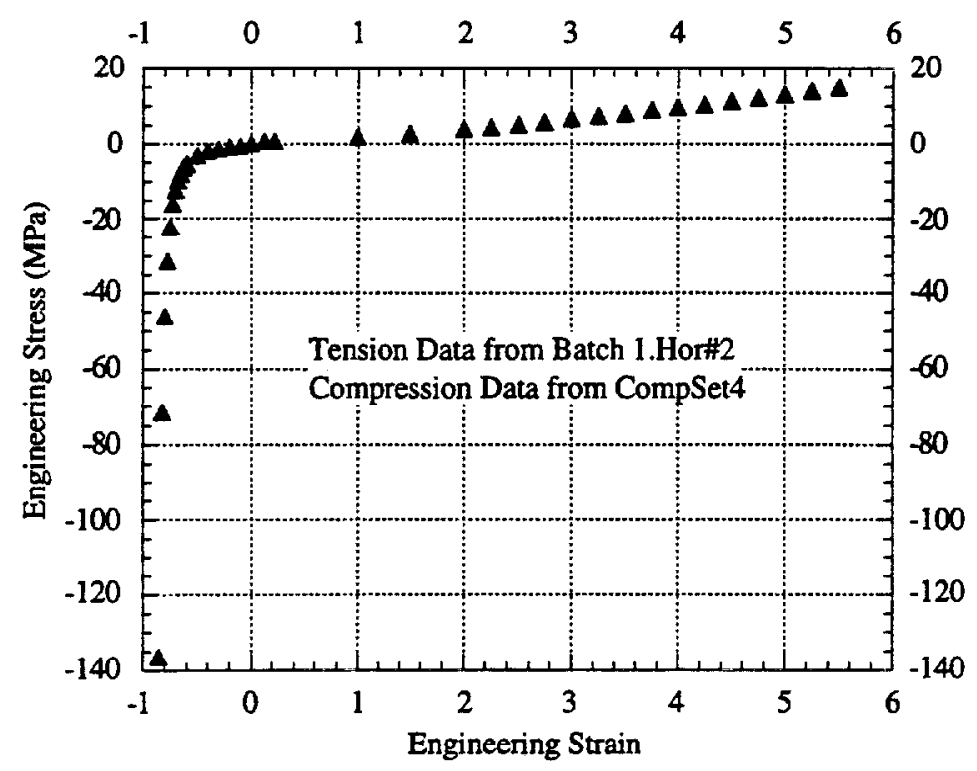

Figure 3.12 - Rubber model 1 (RM1)

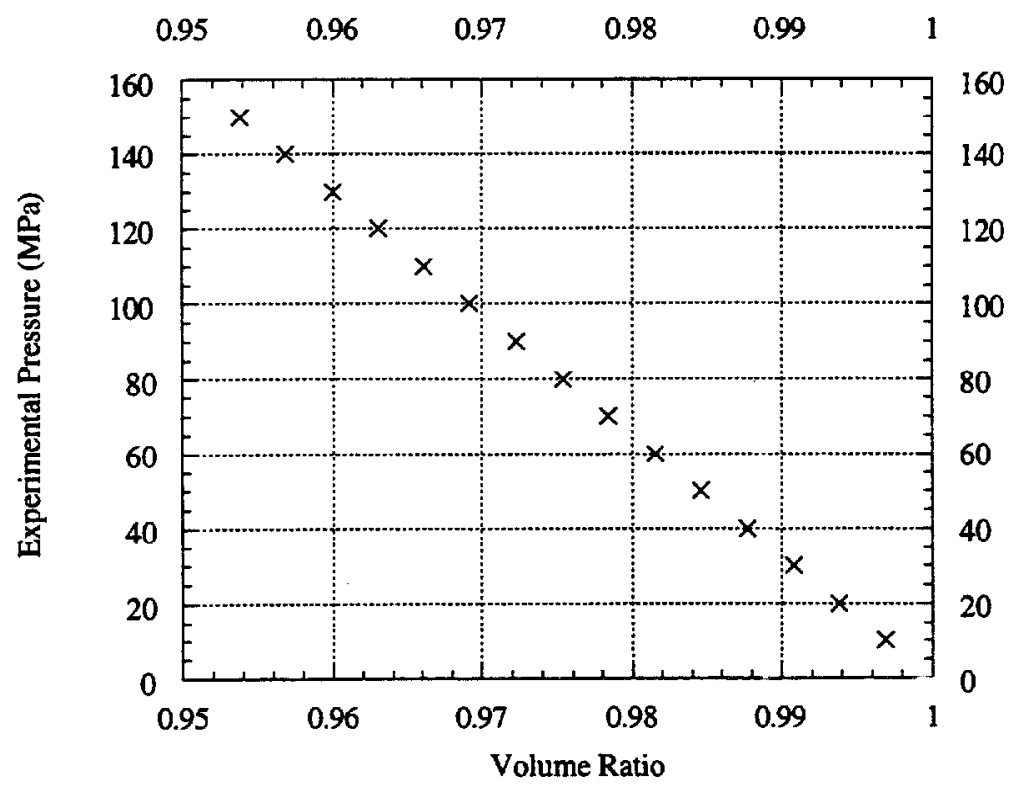

Figure 3.13 - Volumetric compression plot (based on CS7) 


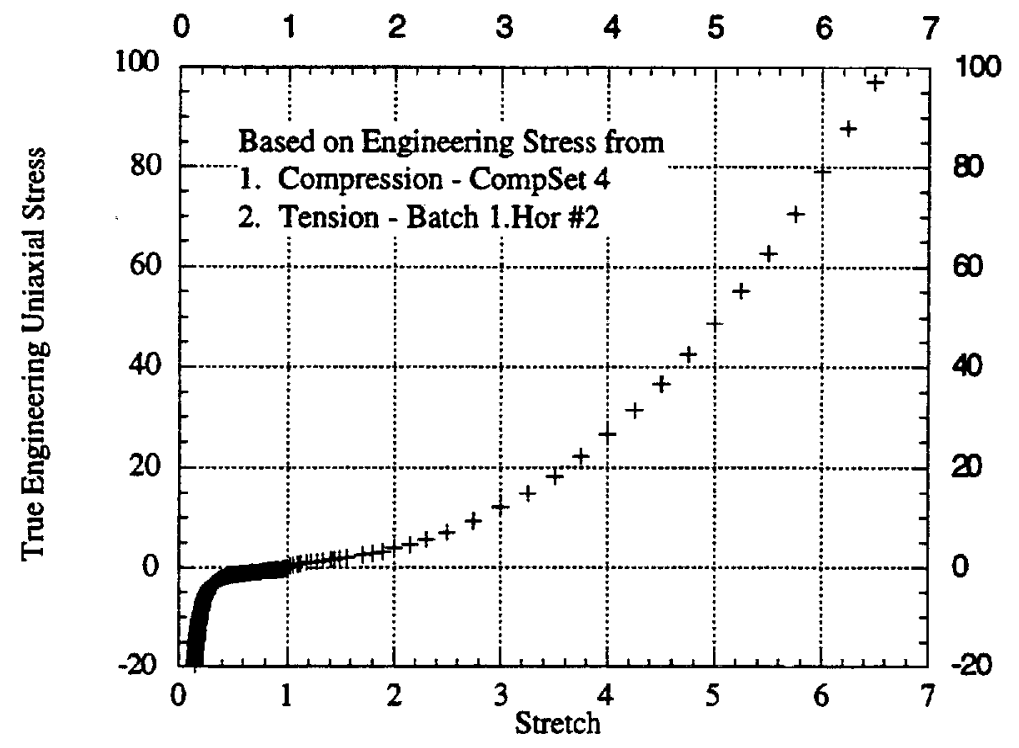

Figure 3.14 - True stress-strain data set used to find the derivative of the ValanisLandel Function $\left(u^{\prime}(\lambda)\right)$ 


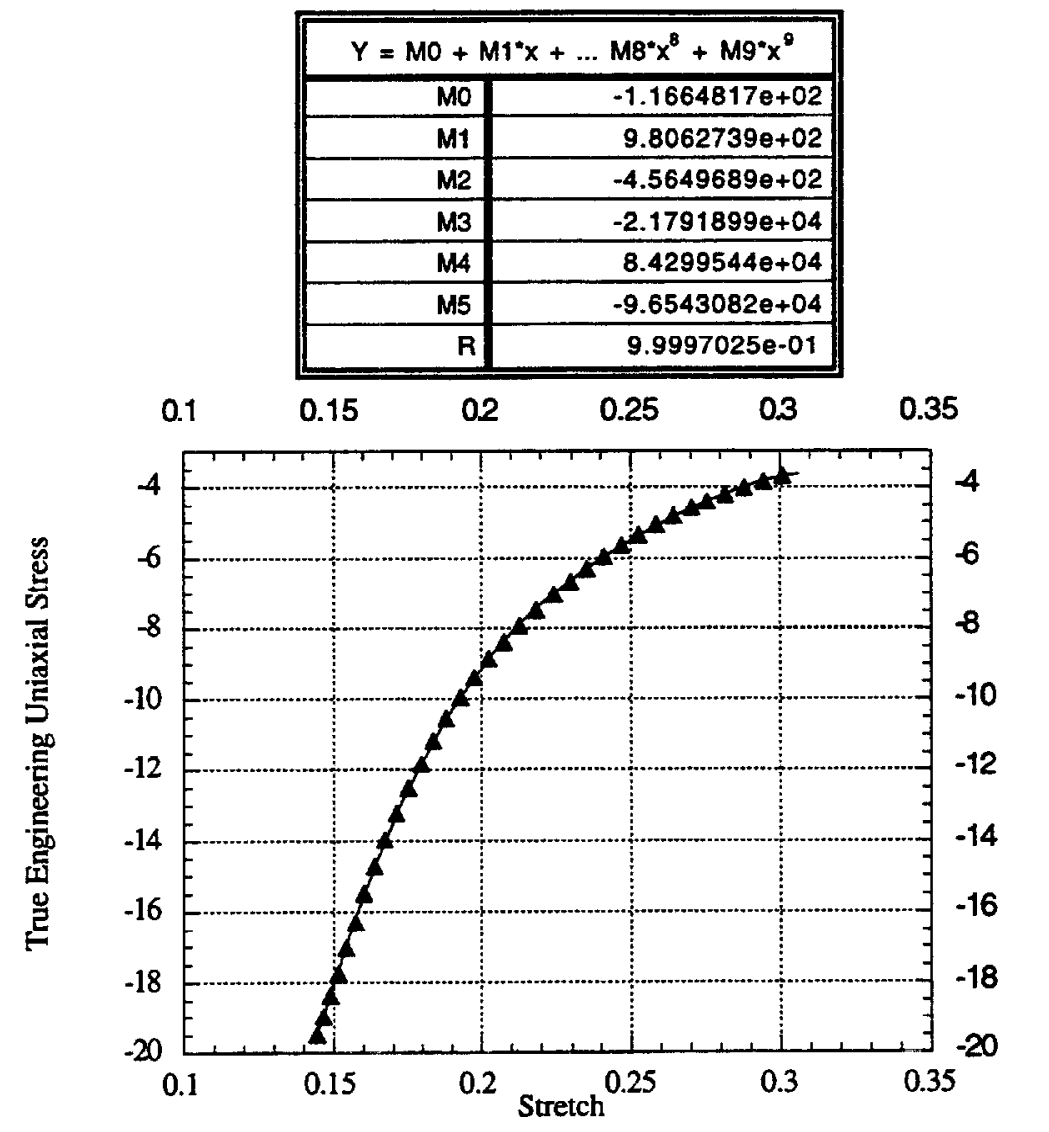

Figure 3.15 - Polynomial curvefit used to obtain a functional representation of the stress-strain curve for determination of the derivative of the ValanisLandel Function $\left(u^{\prime}(\lambda)\right)$ for $0.14<\lambda<0.3$ 


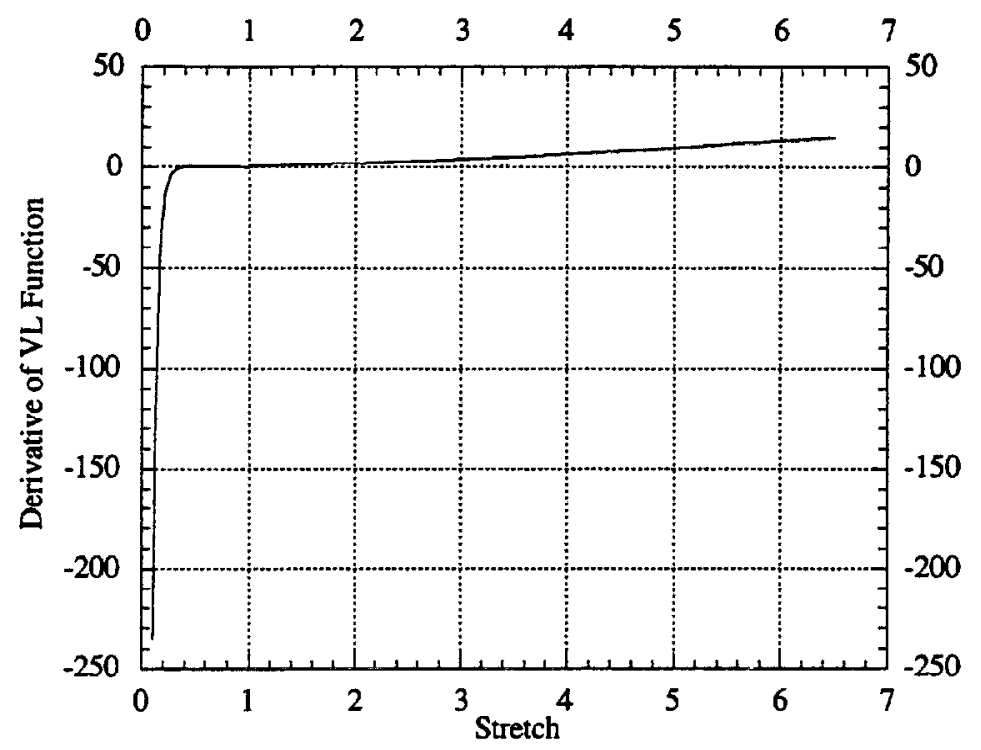

Figure 3.16 - Derivative of the Valanis-Landel Function $\left(u^{\prime}(\lambda)\right)$

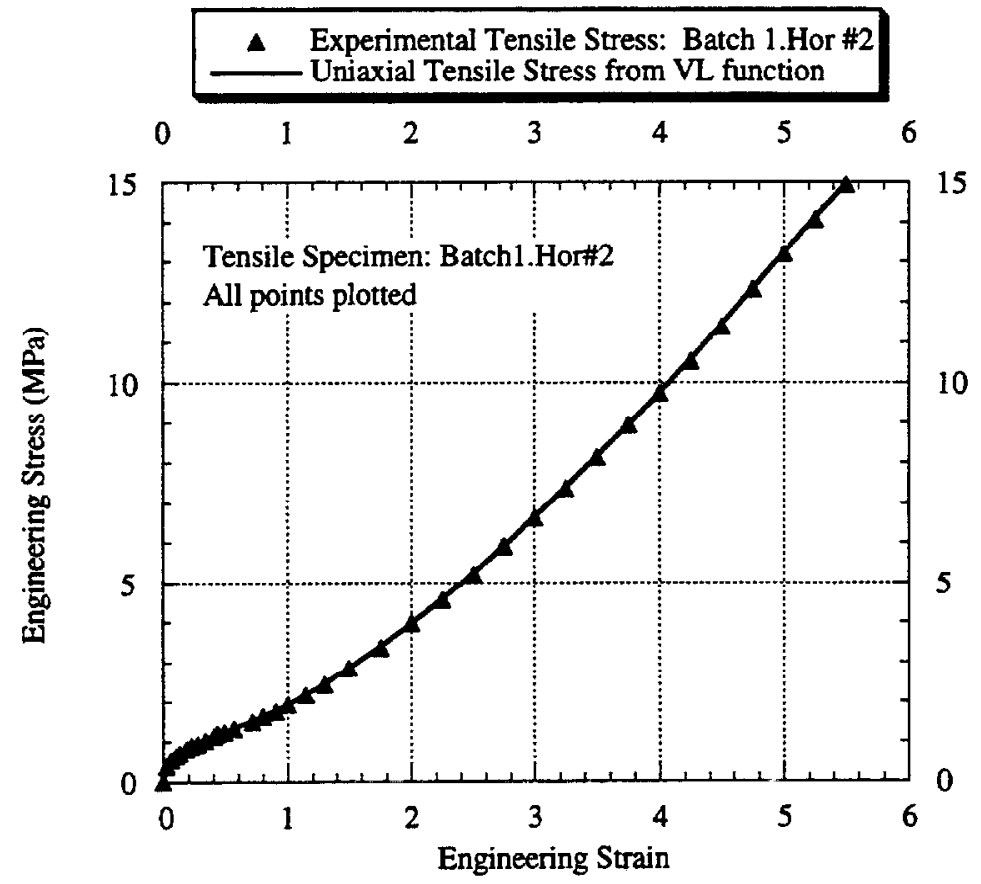

Figure 3.17 - Comparison between the experimental uniaxial tension test and the uniaxial tension derived from the Valanis-Landel function 


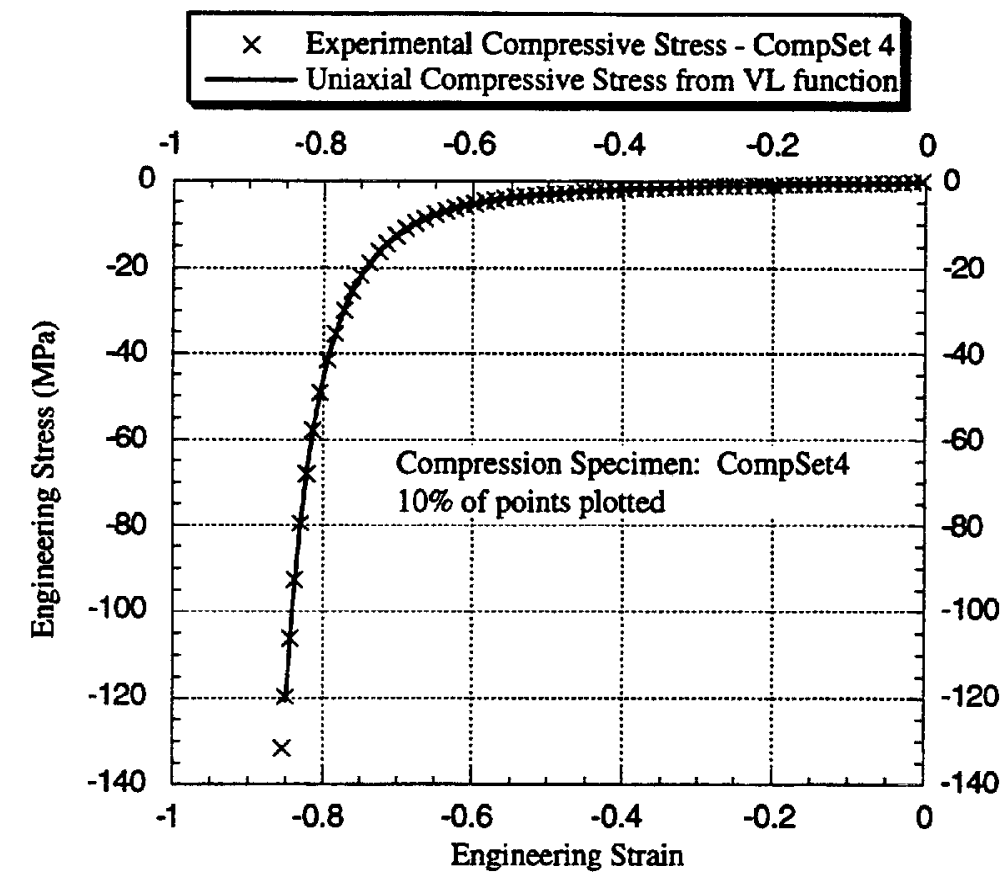

Figure 3.18 - Comparison between the experimental uniaxial compression test and the uniaxial compression derived from the Valanis-Landel function

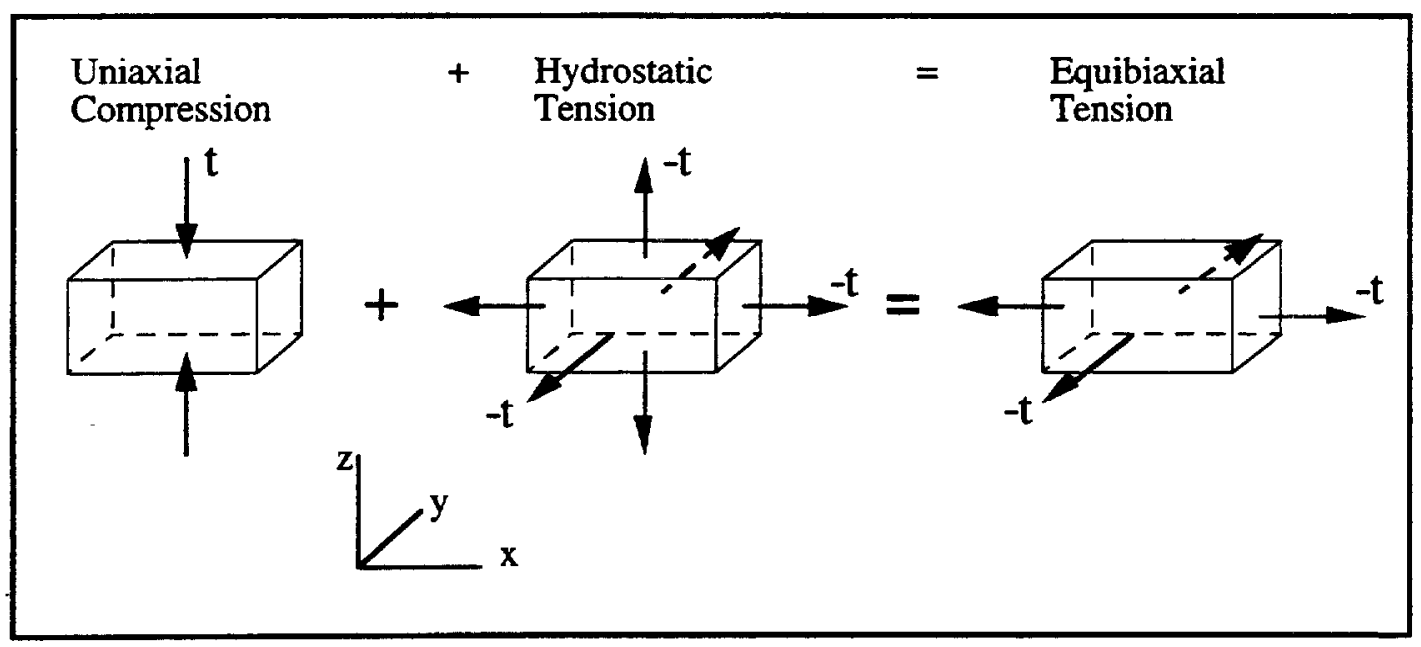

Figure 3.19 - Equivalence of uniaxial compression and equibiaxial tension for an incompressible material 


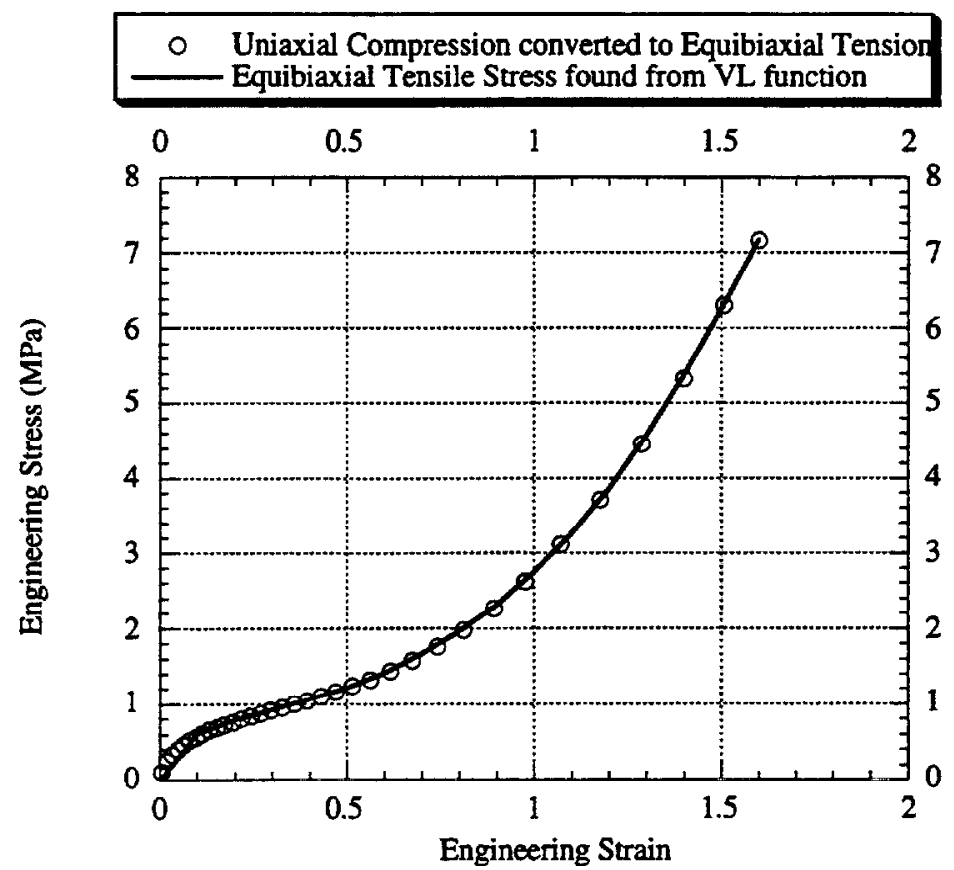

Figure 3.20 - Comparison between the equibiaxial tension converted from uniaxial compression and the equibiaxial tension derived from the Valanis-

Landel function

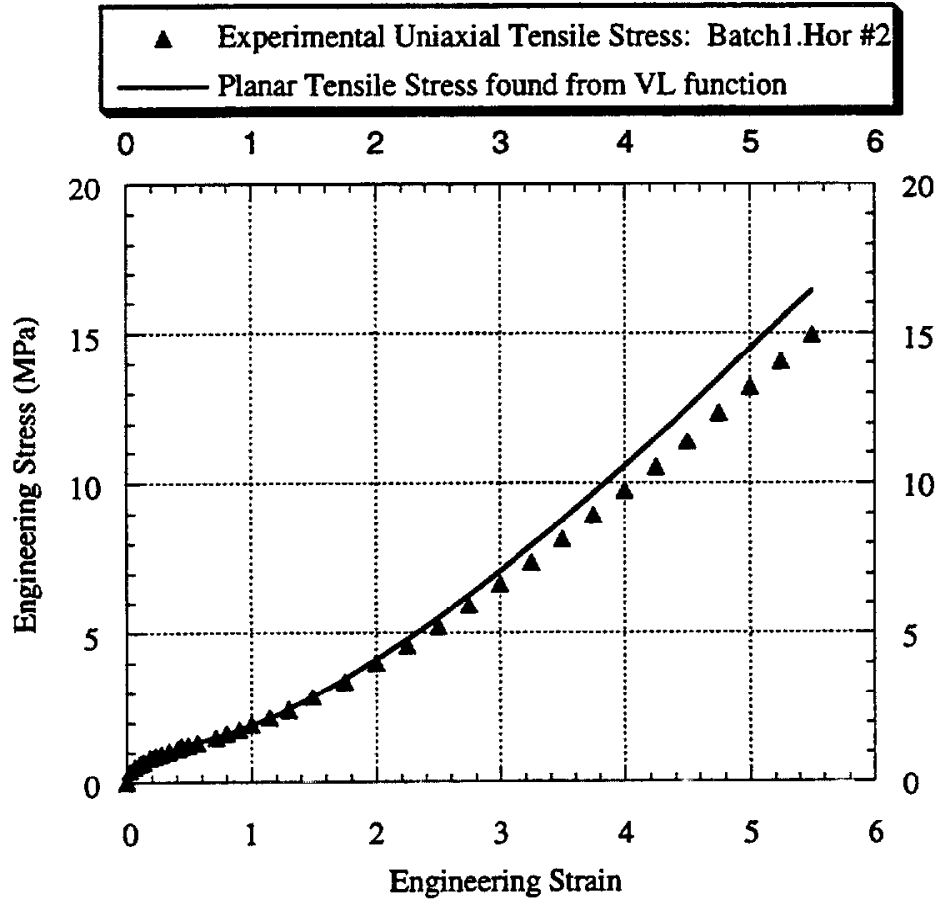

Figure 3.21 - Comparison between the experimental uniaxial tension test and the planar tension derived from the Valanis-Landel function 


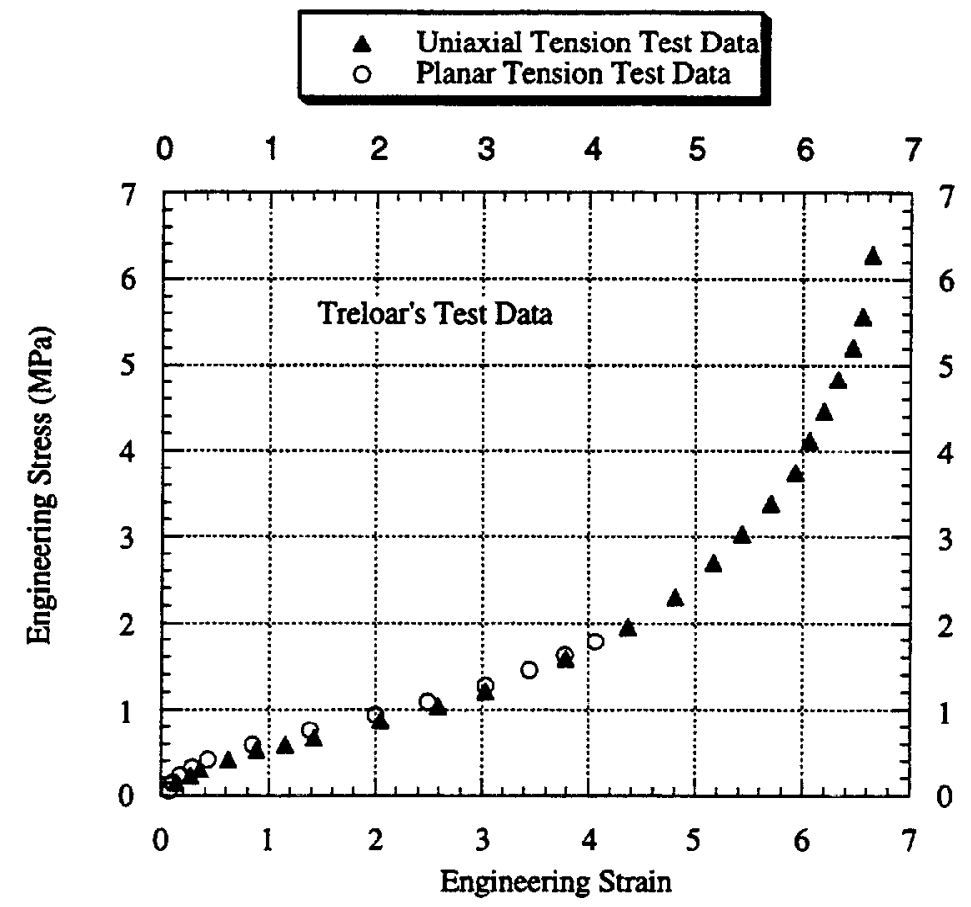

Figure 3.22 - Treloar's experimental data

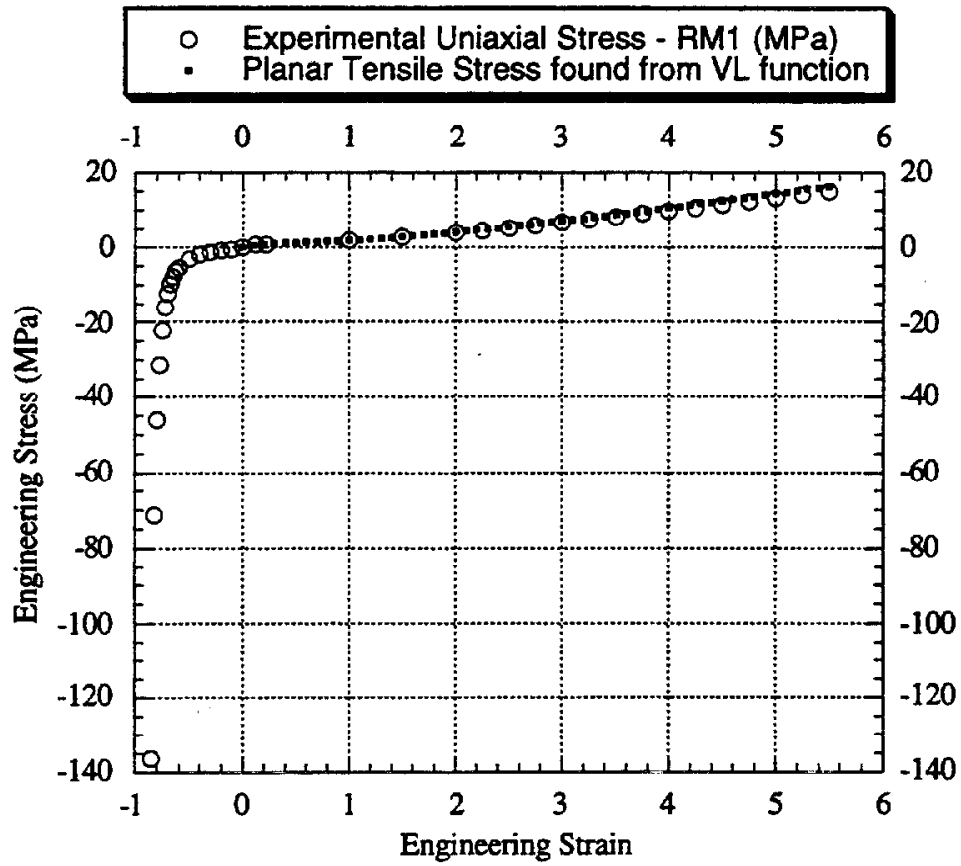

Figure 3.23 - VL1 rubber model 


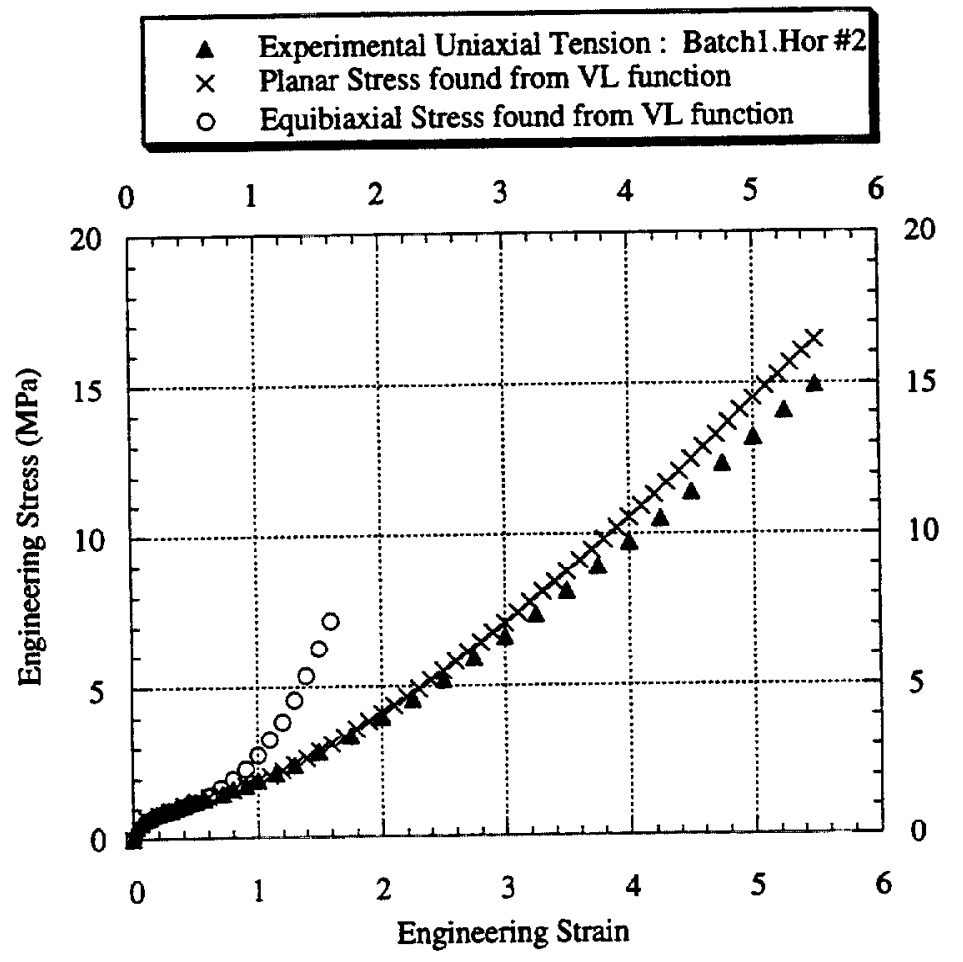

Figure 3.24 - VL2 rubber model 


\section{BLANK PAGE}




\section{INVESTIGATION OF THE RUBBER MODELS USED IN THE FINITE ELEMENT ANALYSIS}

\subsection{Calibration of the Rubber Models in ABAQUS}

To validate the finite element modeling of rubber, we need to see how well the finite element results approximate the experimental and Valanis-Landel generated stress-strain curves. In this section we select the number of terms to be included in each strain energy function, and the element type to be used in the finite element analysis. The selected element has been subjected to the proper boundary conditions to reproduce each of the four modes we are analyzing, namely uniaxial tension, uniaxial compression, equibiaxial tension, and planar tension. At the end of this section, the finite element results are compared directly to the experimental data and the data generated by the Valanis-Landel function for equibiaxial and planar tension.

To determine how many terms from the strain energy potentials to include in the analysis, it is helpful to look at the reduced stress. For deformations in uniaxial tension and compression, the true stress is

$$
t=2\left(\lambda^{2}-\frac{1}{\lambda}\right)\left(\frac{\partial U}{\partial I_{1}}+\frac{1}{\lambda} \frac{\partial U}{\partial I_{2}}\right)
$$

Dividing Equation (4.1) through by $\lambda^{2}-\frac{1}{\lambda}$ defines a measure called the "reduced" stress (McKenna and Zapas, 1983; Yeoh, 1993), shown as

$$
t_{R} \equiv \frac{t}{\left(\lambda^{2}-1 / \lambda\right)}=2\left(\frac{\partial U}{\partial I_{1}}+\frac{1}{\lambda} \frac{\partial U}{\partial I_{2}}\right)
$$

The terms on the right-hand side provide a sensitive measure of the deviations of the elastic properties from constant values (McKenna and Zapas 1983). By plotting $\frac{1}{\lambda}$ as the abscissa, the compression information is shown in the region greater than 1 , and the tension information is compressed between 0 and 1 .

For the Polynomial Strain Energy Potential, $\mathrm{N}=1$ is the Mooney-Rivlin model. Written out explicitly, we get

$$
U=C_{10}\left(I_{1}-3\right)+C_{01}\left(I_{2}-3\right)
$$

Taking the appropriate derivatives of Equation (4.3) and inserting them into Equation (4.2), the reduced stress-strain relation for uniaxial tension and compression is

$$
t_{R}=2 C_{10}+\frac{1}{\lambda} 2 C_{01}
$$


Equation (4.4) shows that the Mooney-Rivlin model will produce reduced stresses that are linear when plotted against $\frac{1}{\lambda}$. Figure 4.1 shows the experimental uniaxial tensile and compressive stresses expressed as reduced stresses and plotted versus $\frac{1}{\lambda}$. It is clear that a linear approximation will not be sufficient to properly model the rubber. For this reason, the calibration runs were performed using $\mathrm{N}=2$ for the Polynomial Strain Energy Potential.

This type of analysis is not so easily done with the Ogden Strain Energy Potential. The Ogden model is written in terms of the stretches, not the strain invariants. However, the larger the number of terms included $(\mathrm{N})$, the easier the model should capture the nonlinearity in the stress-strain curve. Due to the high nonlinearity present in the experimental data, the calibration runs used $N=6$, which is the largest number of terms available in the ABAQUS code. Lower values of $N$ require less computational effort to obtain the stresses, so, for comparison, the Ogden model with $\mathrm{N}=3$ was tested. In Section 4.2 , the calibration runs for the uniaxial deformation mode will be shown in terms of reduced stresses.

The uniaxial, equibiaxial, and planar calibration runs were conducted using one 3-dimensional, linear displacement "hybrid" finite element with reduced integration (ABAQUS element type C3D8RH), with unit dimensions in $\mathrm{X}, \mathrm{Y}$, and $\mathrm{Z}$. Elements in ABAQUS that use the mixed formulation (c.f. Section 3.2) are called hybrid elements. Proper application of boundary conditions allows us to perfectly simulate these homogeneous deformations. The use of one finite element with an assumed linear displacement is sufficient, since homogeneous deformations have a constant strain field and a single element with an assumed linear displacement can represent this state of strain.

For each of the four deformation modes, compressible and incompressible analyses were carried out. Table 4.1 shows the calibration runs performed. CompSet 7 (CS7 - see Figure 3.13) was the volumetric compression data used for all the Compressibility runs. RM1 is shown in Figure 3.12, the VL1 rubber model in Figure 3.23, and the VL2 rubber model in Figure 3.24. 
Table 4.1 - Rubber calibrations

\begin{tabular}{ccc}
\hline Type of Test & $\begin{array}{c}\text { Compressible Models } \\
\text { (uses CS7) }\end{array}$ & $\begin{array}{c}\text { Incompressible Models } \\
\text { (excludes CS7) }\end{array}$ \\
\hline Uniaxial Tension & RM1, VL1, VL2 & RM1 \\
Uniaxial Compression & RM1, VL1, VL2 & RM1 \\
Equibiaxial Tension & RM1, VL1, VL2 & RM1 \\
Planar Tension & RM1, VL1, VL2 & RM1 \\
Volumetric Compression & RM1, VL1 & Not Applicable \\
\hline
\end{tabular}

Note: All tests have been conducted using the Ogden Strain Energy Potential, $N=6$ and $\mathrm{N}=3$, and the Polynomial Strain Energy Potential, $\mathrm{N}=2$, except the Volumetric Test, in which only the Ogden form, $N=6$ was run.

For the volumetric compression calibration runs, an axisymmetric finite element model was used. This model is appropriate since the volumetric specimen is circular and the loading is uniformly distributed. A slice of the specimen in the R-Z plane is modeled, from $R=0$ to the outer edge and from the midheight to the top, so that $1 / 4$ of a slice through the axis of symmetry is modeled. CAX $4 \mathrm{H}$ elements were used, which are hybrid 2-dimensional linear displacement elements. Fixed boundary conditions were set along the midheight nodes in both the $\mathrm{R}$ and $\mathrm{Z}$ directions, and along the outer edge and the top nodes in the $\mathrm{R}$ direction. The axisymmetric element automatically applies fixed boundary conditions along $R=0$. Displacement boundary conditions were placed on the top nodes, moving each node down by the same amount (the negative $\mathrm{Z}$ direction), and the RIKS arc-length method was used. The model converged rapidly.

As shown in Table 4.1, for the uniaxial, equibiaxial, and planar modes of deformation, the calibrations were done with and without compressibility, for rubber model RM1. The finite element results are virtually identical whether or not compressibility is included, so the VL1 and VL2 calibrations were run using only a compressible model. The similarity between compressible and incompressible results is because the homogeneous deformations allow the rubber to freely expand or contract, so as to nearly retain its volume. Hence the volumetric deformation is negligibly small.

The RM1, VL1, and VL2 rubber models are used to determine the deviatoric parameters. For purposes of comparing the results by model, it is important to keep in mind which deformation modes are included in each of the models. Table 4.2 summarizes the test data included in each model. Following this table are average Root Mean Square (RMS) errors for each calibration run and plots of the results, organized by deformation mode. 
Table 4.2 - Test data included in each rubber model

\begin{tabular}{cccc}
\hline Deformation Mode & \multicolumn{3}{c}{ Rubber Models } \\
& RM1 & VL1 & VL2 \\
\hline Uniaxial Tension & $\mathrm{X}$ & $\mathrm{X}$ & $\mathrm{X}$ \\
Uniaxial Compression & $\mathrm{X}$ & $\mathrm{X}$ & \\
Equibiaxial Tension & & & $\mathrm{X}$ \\
Planar Tension & & $\mathrm{X}$ & $\mathrm{X}$ \\
\hline
\end{tabular}

The average RMS error is calculated from the formula shown in Equation (4.5), and measures the relative error in stress.

$$
\text { avg } R M S \text { error }=\frac{1}{M} \sqrt{\sum_{i=1}^{N}\left(\frac{\sigma_{\exp }-\sigma_{F E}}{\sigma_{\exp }}\right)^{2}}
$$

$M$ is the number of points at which the stress was calculated by the finite element analysis, $\sigma_{F E}$ is the stress calculated by the finite element analysis, and $\sigma_{\text {exp }}$ is the experimentally determined stress.

Table 4.3 shows the average RMS error for each of the calibration runs for Uniaxial Tension. The performance of each model is good, with slightly better results from rubber models VL1 and VL2. Figures 4.2 through 4.5 are plots of the finite element results versus the experimental tension data, and graphically depict the closeness of the finite element approximations to the tensile data, using both experimental data and data generated by the Valanis-Landel function.

Table 4.3 - Average RMS error of calibration runs for uniaxial tension

\begin{tabular}{ccccc}
\hline \multicolumn{2}{c}{ Rubber Model } & Ogden (N=6) & Ogden (N=3) & Poly. (N=2) \\
\hline RM1 & Compressible & 0.0195 & 0.0159 & 0.0177 \\
RM1 & Incompressible & 0.0195 & 0.0159 & 0.0177 \\
VL1 & Compressible & 0.0119 & 0.0160 & 0.0195 \\
VL2 & Compressible & 0.0117 & 0.0162 & 0.0156 \\
\hline
\end{tabular}

Table 4.4 shows the average RMS error for each of the calibration runs for Uniaxial Compression. Each finite element result provides a good approximation to the experimental compression data, with RM1 slightly better. Also, note that the 
Ogden model with 6 terms $(\mathrm{N}=6)$ results in the lowest average RMS error for each rubber model. Figures 4.6 through 4.9 are plots of the finite element results versus the experimental compression data.

Table 4.4 - Average RMS error of calibration runs for uniaxial compression

\begin{tabular}{ccccc}
\hline \multicolumn{2}{c}{ Rubber Model } & Ogden $(\mathrm{N}=6)$ & Ogden $(\mathrm{N}=3)$ & Poly. (N=2) \\
\hline RM1 & Compressible & 0.0280 & 0.0446 & 0.0476 \\
RM1 & Incompressible & 0.0280 & 0.0446 & 0.0476 \\
VL1 & Compressible & 0.0305 & 0.0451 & 0.0508 \\
VL2 & Compressible & 0.0309 & 0.0452 & 0.0417 \\
\hline
\end{tabular}

Table 4.5 shows the average RMS error for each of the calibration runs for equibiaxial tension, and Figures 4.10 through 4.13 show plots of each run. The comparisons are between the data generated by the Valanis-Landel function and the ABAQUS results using the three rubber models. The results for each rubber model are comparable, but experimental rubber model RM1 does a slightly better job than VL1 and VL2 when using the Ogden, $\mathrm{N}=6$ and Polynomial, $\mathrm{N}=2$ strain energy functions, with the Ogden, $N=6$ providing the best fit to the equibiaxial data generated by the Valanis-Landel function. It is important to note that rubber models RM1 and VL1 do not include the equibiaxial data set, yet ABAQUS closely approximates the equibiaxial deformation mode.

Table 4.5 - Average RMS error of calibration runs for equibiaxial tension

\begin{tabular}{ccccc}
\hline \multicolumn{2}{c}{ Rubber Model } & Ogden (N=6) & Ogden (N=3) & Poly. (N=2) \\
\hline RM1 & Compressible & 0.0117 & 0.0271 & 0.0226 \\
RM1 & Incompressible & 0.0118 & 0.0268 & 0.0225 \\
VL1 & Compressible & 0.0135 & 0.0273 & 0.0389 \\
VL2 & Compressible & 0.0133 & 0.0262 & 0.0256 \\
\hline
\end{tabular}

Table 4.6 shows the average RMS error for each of the calibration runs for planar tension, and Figures 4.14 through 4.17 show plots of each run. An important observation is that the Ogden strain energy function, using rubber model RM1 (which does not include the planar data), does a much better job of fitting the planar data than does the Polynomial strain energy function. This is probably due to the fact that the Ogden model is a special form of the Valanis-Landel function. While this alone does not guarantee that the planar data are correct, the planar tensile stresses from the Valanis-Landel function are slightly larger than the experimental uniaxial stresses, and 
this is similar to the relationship between the experimentally determined planar tension data and uniaxial tension data reported by Treloar (1943). A second observation is that when the planar data is included in the data set used to determine the strain energy function (VL1 and VL2), the Polynomial strain energy function performs nearly as well as the Ogden function.

Table 4.6 - Average RMS error of calibration runs for planar tension

\begin{tabular}{lcccc}
\hline \multicolumn{2}{c}{ Rubber Model } & Ogden (N=6) & Ogden (N=3) & Poly. (N=2) \\
\hline RM1 & Compressible & 0.0182 & 0.0147 & 0.0954 \\
RM1 & Incompressible & 0.0181 & 0.0144 & 0.1012 \\
VL1 & Compressible & 0.0100 & 0.0123 & 0.0243 \\
VL2 & Compressible & 0.0097 & 0.0121 & 0.0165 \\
\hline
\end{tabular}

The volumetric test runs were only performed using the compressibility data (CS7) for rubber models RM1 and VL1. Using an incompressible model would result in no deformation, since the rubber is perfectly confined. As shown in Figure 4.18, the finite element program accurately reproduces the experimental data, and as expected, there is no difference between the use of either RM1 or VL1, because those models determine the deviatoric parameters, whereas data from CS7 is used to determine the volumetric parameters and is the same for both calibration runs.

\subsection{Reduced Stress Plots}

As noted in Section 4.1, the reduced stress provides a sensitive measure of the deviations of the elastic properties from constant values. Reduced stress plots, also referred to as Mooney-Rivlin plots, are frequently used by researchers who study the mechanical properties of rubber. The formula for reduced uniaxial stress is repeated here for convenience.

$$
t_{R} \equiv \frac{t}{\left(\lambda^{2}-1 / \lambda\right)}=2\left(\frac{\partial U}{\partial I_{1}}+\frac{1}{\lambda} \frac{\partial U}{\partial I_{2}}\right)
$$

By plotting $\frac{1}{\lambda}$ as the abscissa, the compression information is shown in the region greater than 1 , and the tension information is compressed between 0 and 1 .

In this section, the calibration results for uniaxial stress are presented in terms of the reduced stresses. Figures 4.19 through 4.21 show plots of the reduced stress versus $\frac{1}{\lambda}$ for the RM1, VL, and VL2 rubber models, respectively. These plots mirror the results shown in the calibration runs in Section 4.1. 
The experimental data exhibit a rapid increase in modulus as the unstrained state is approached. This has been observed by McKenna and Zapas (1983) and other authors. Finite element results do not capture this change in reduced stress. McKenna and Zapas reported that the compression modulus is greater than the tension modulus near the undeformed state. This observation agrees with our experimental data. The reasons for this phenomenon are not clear. McKenna and Zapas (1983) used bonded cylinders and corrected the results to homogeneous compression using factors determined in a finite difference analysis. They conjectured that this phenomenon might be due to their experimental approach. However, in our experiments, the tests were carried out on unbonded cylinders that had been greased to reduce friction along the loaded surfaces.

It should be noted that most work in rubber using the VL function and Mooney-Rivlin plots has been done with unfilled rubber. However, the rubber used in this testing program is filled with carbon black. This filler was added primarily to control the shear stiffness and damping characteristics. The Mooney-Rivlin plots of unfilled rubbers exhibit different behavior than is observed here. With unfilled rubbers, the response starts at a low value, goes toward a maximum in the vicinity of $1 / \lambda=1.25$ and then decreases mildly before, possibly, increasing again. Here the response at values of $1 / \lambda>1.25$ is strongly concave upwards. 


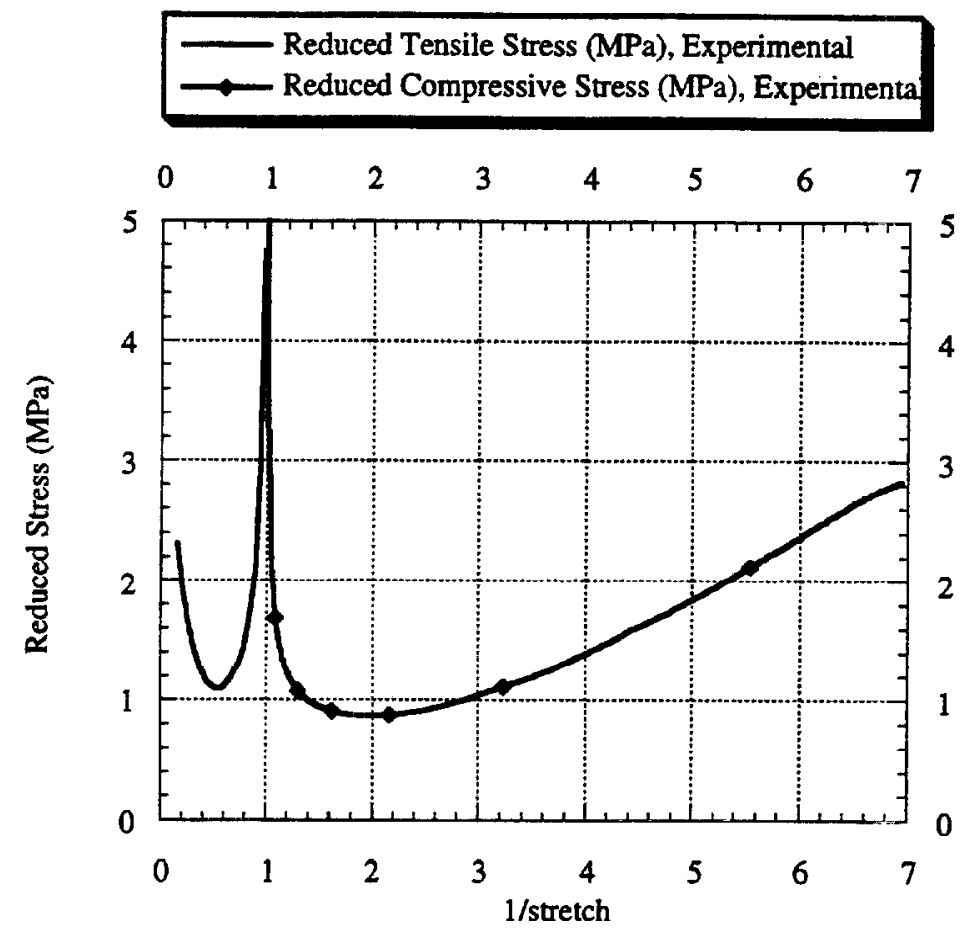

Figure 4.1 - Plot of reduced stresses for the experimental uniaxial tensile and compressive stress-strain data 


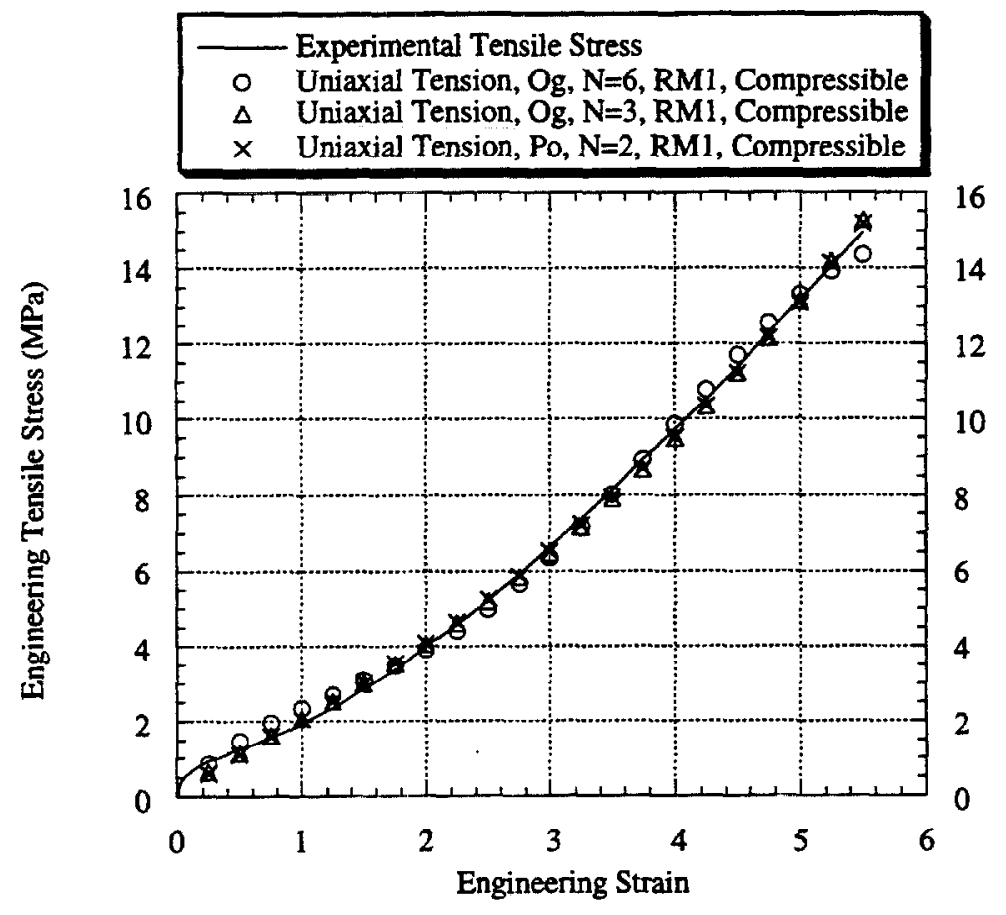

Figure 4.2 - Calibration to uniaxial tension with compressibility using RM1

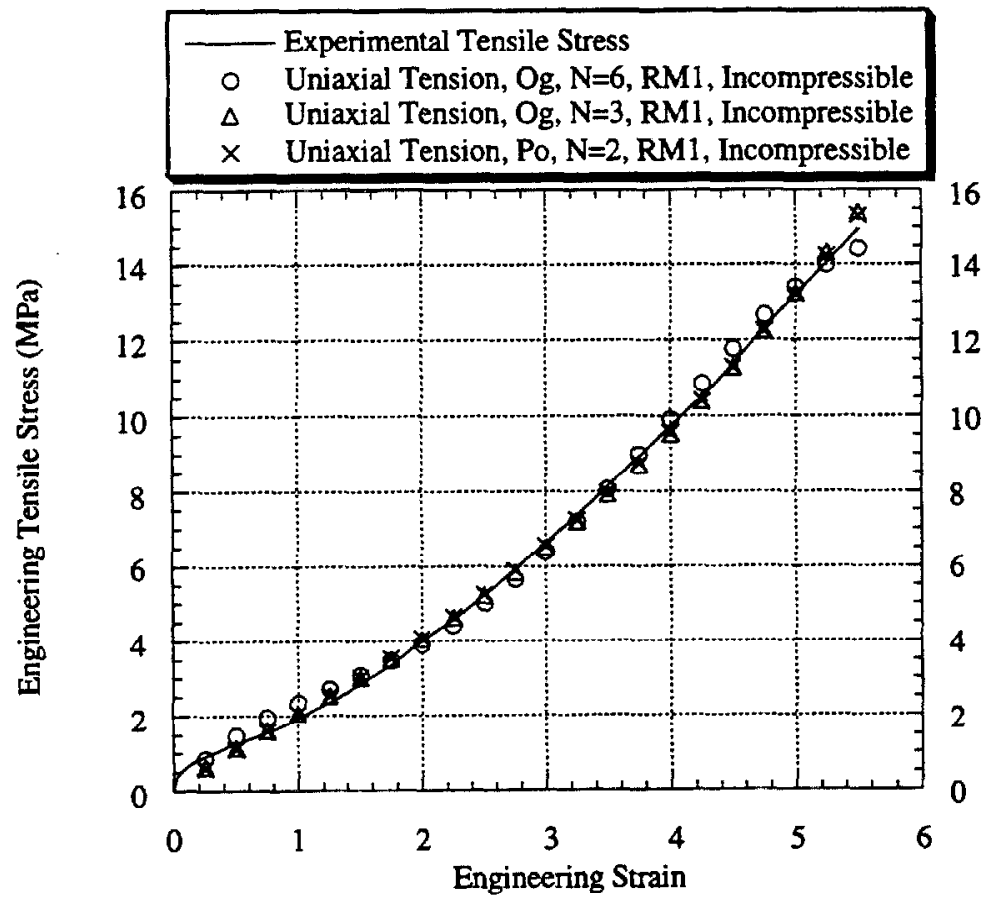

Figure 4.3 - Calibration to uniaxial tension without compressibility using RM1 


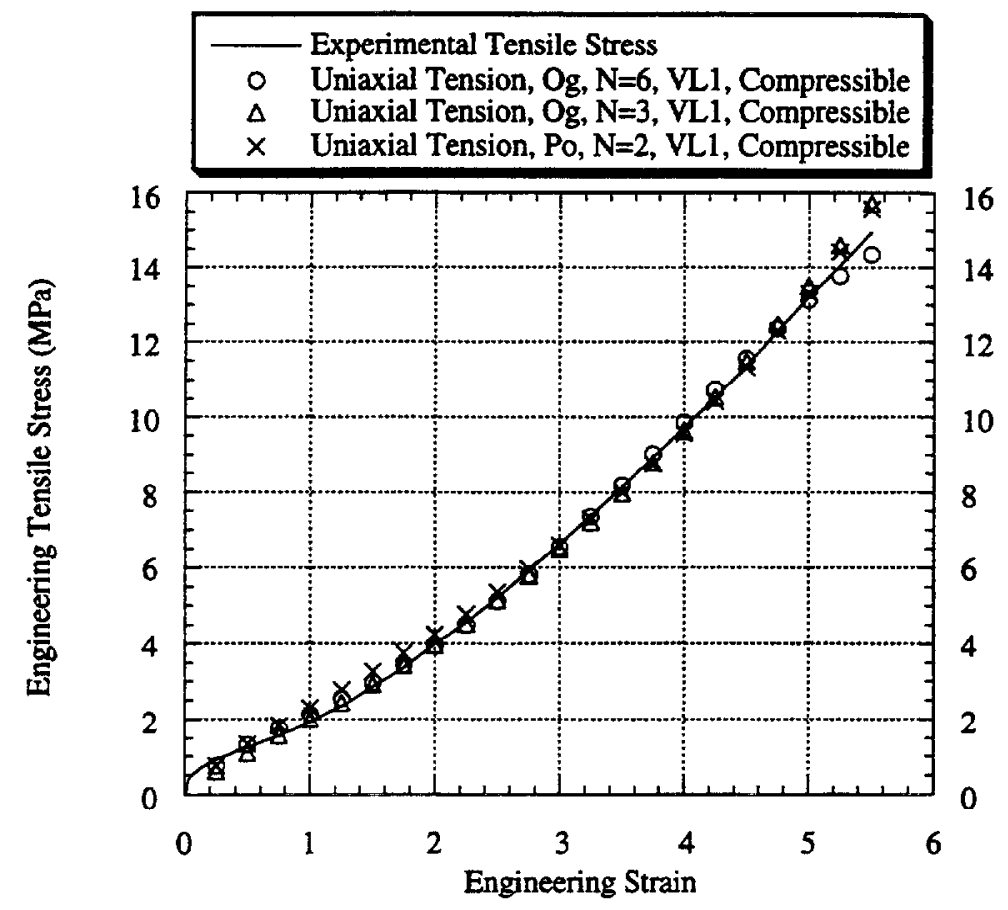

Figure 4.4 - Calibration to uniaxial tension with compressibility using VL1

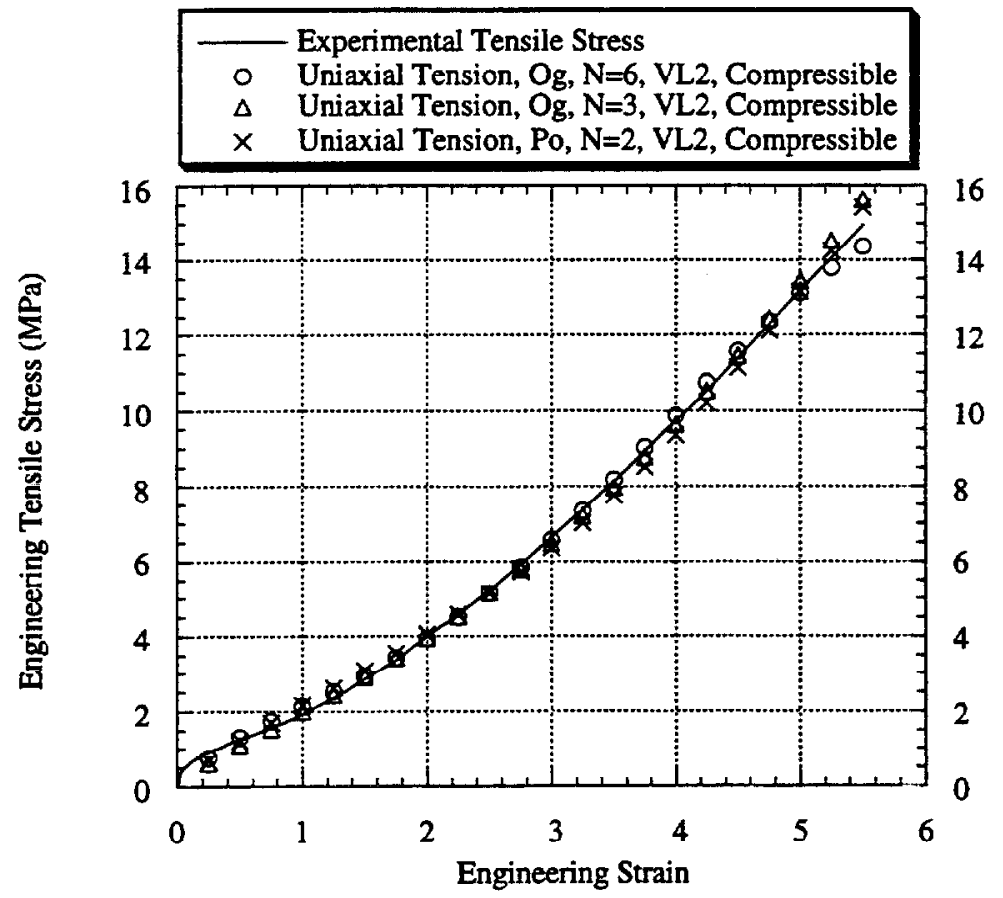

Figure 4.5 - Calibration to uniaxial tension with compressibility using VL2 


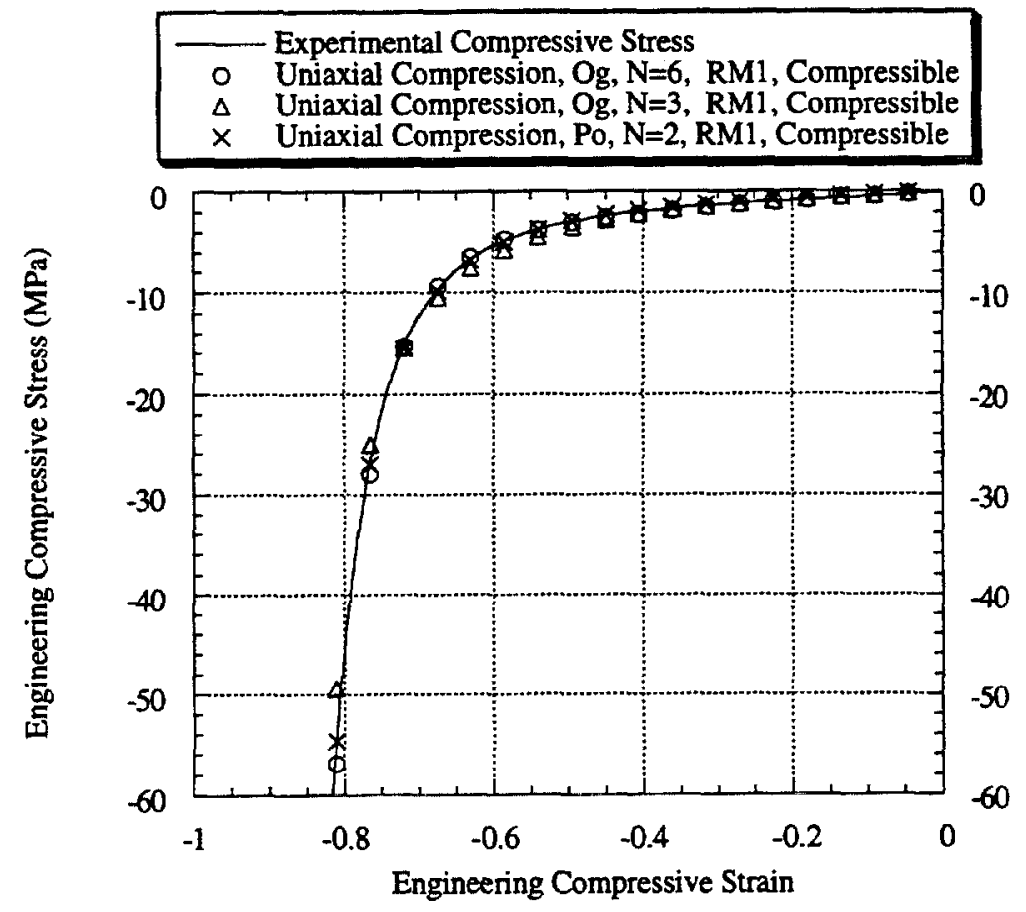

Figure 4.6 - Calibration to uniaxial compression with compressibility using RM1

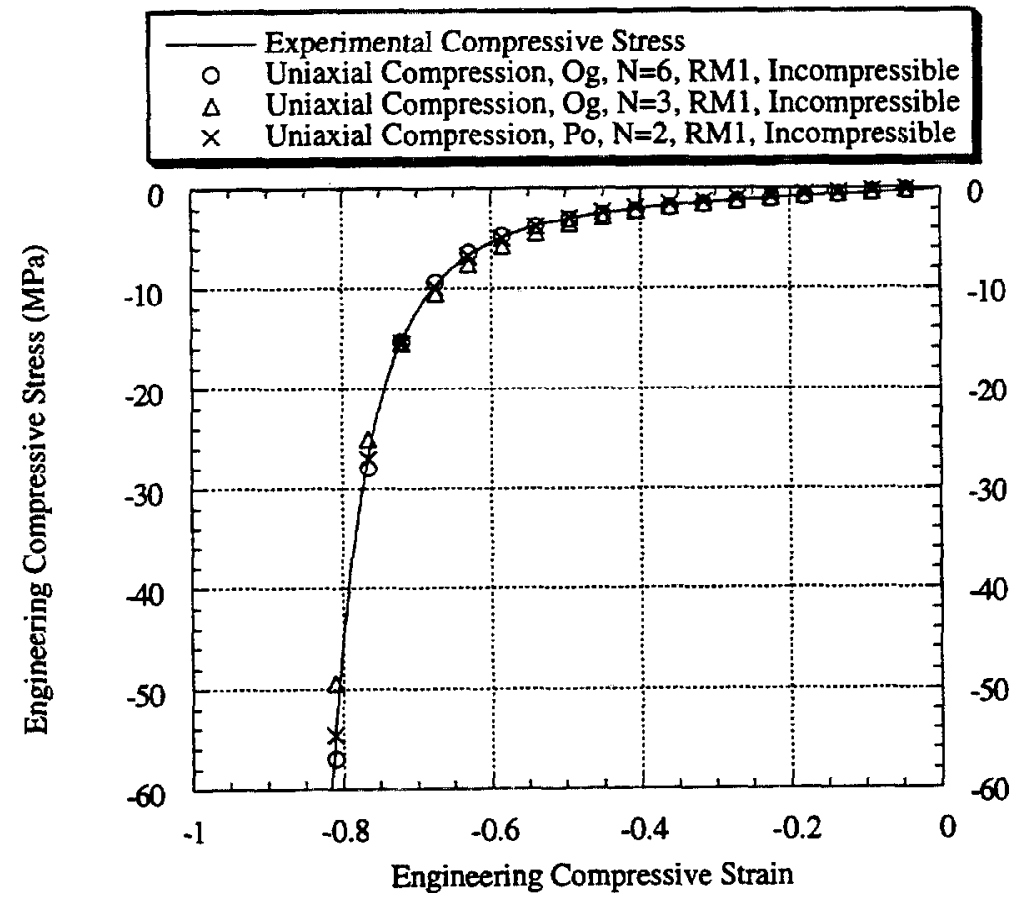

Figure 4.7 - Calibration to uniaxial compression without compressibility using RM1 


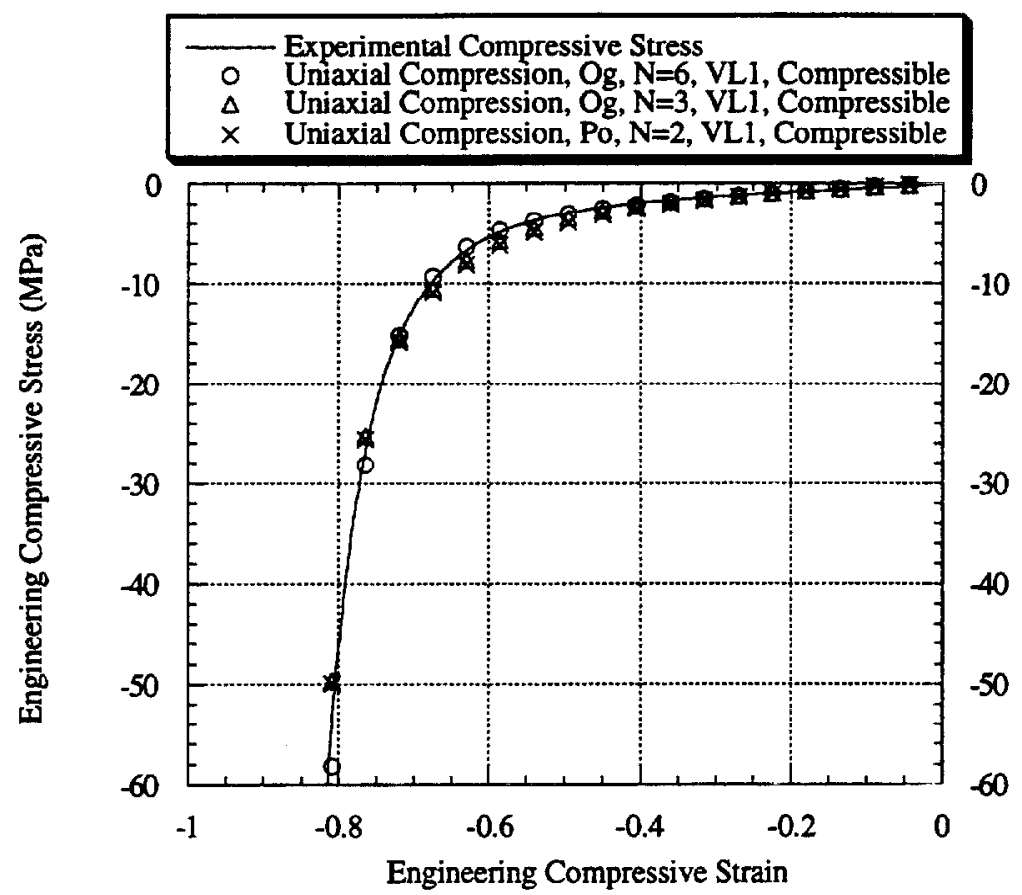

Figure 4.8 - Calibration to uniaxial compression with compressibility using VL1

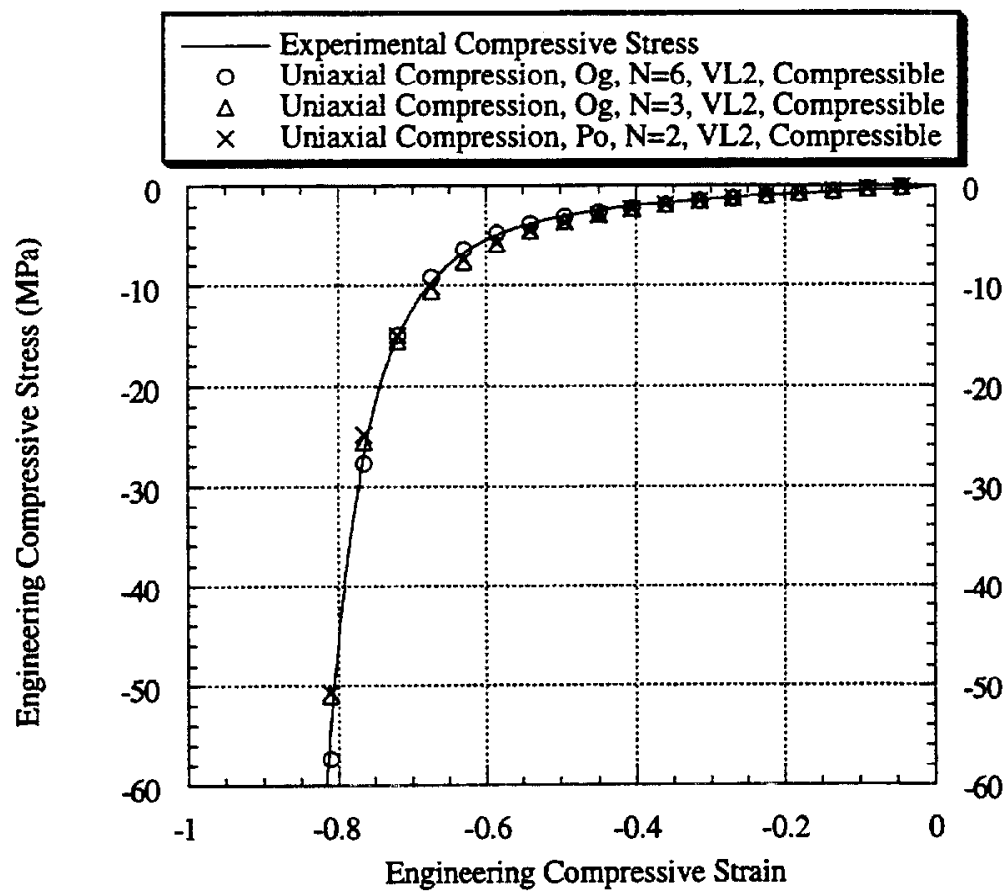

Figure 4.9 - Calibration to uniaxial compression with compressibility using VL2 


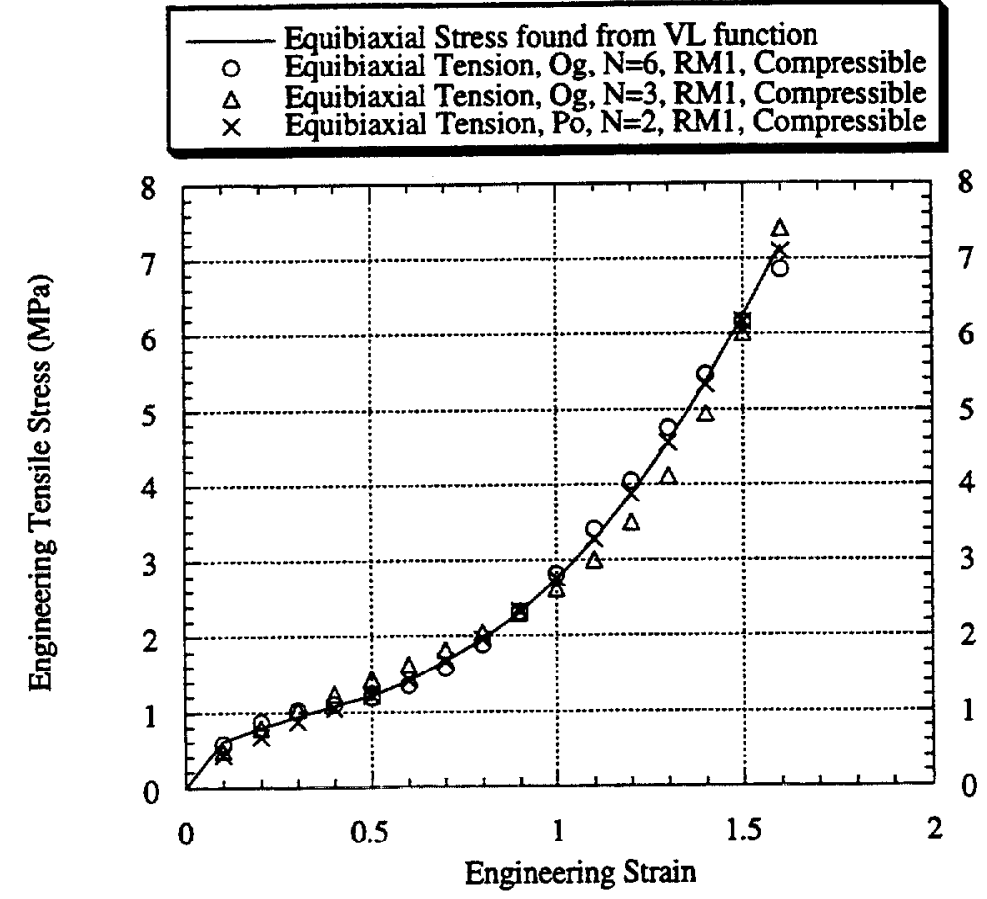

Figure 4.10 - Calibration to equibiaxial tension with compressibility using RM1

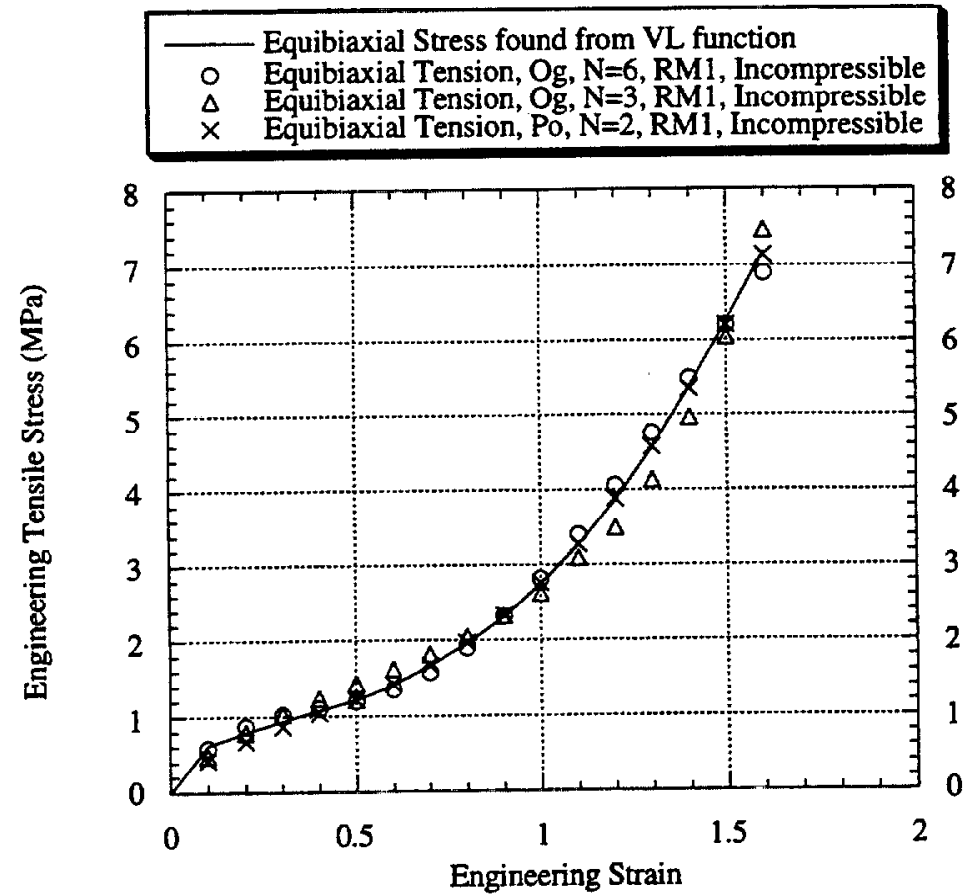

Figure 4.11 - Calibration to equibiaxial tension without compressibility using RM1 


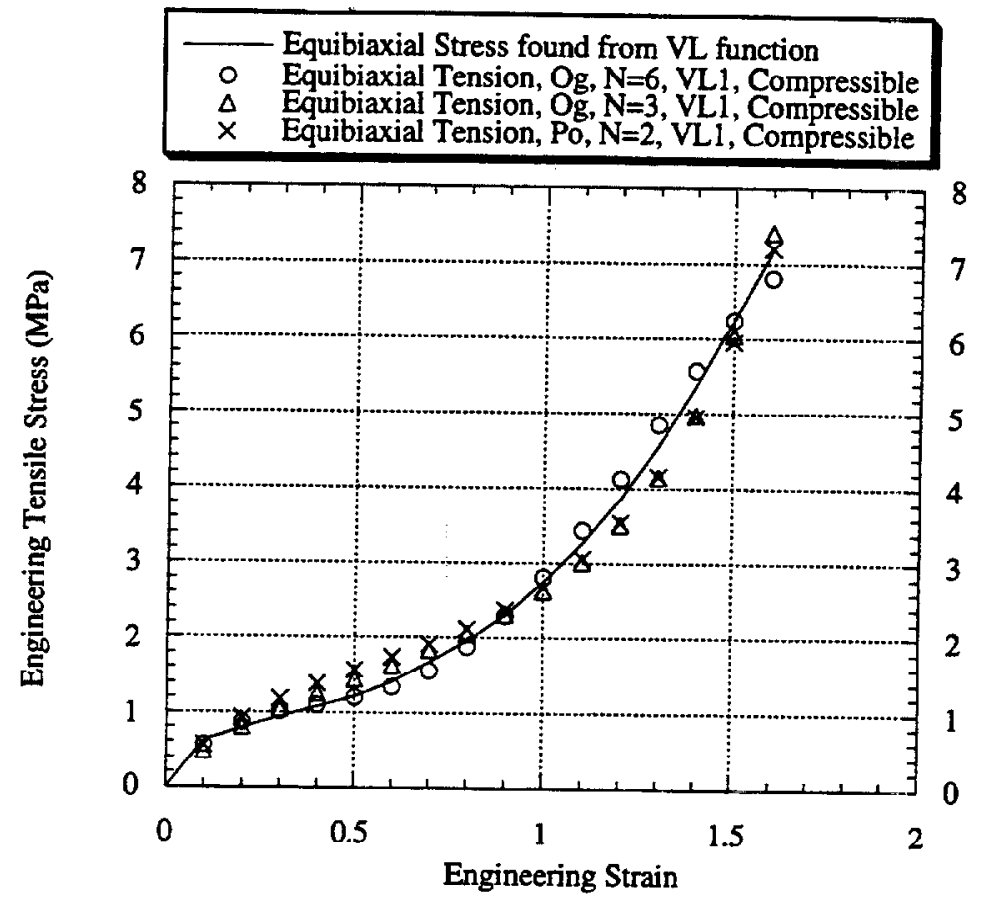

Figure 4.12 - Calibration to equibiaxial tension with compressibility using VL1

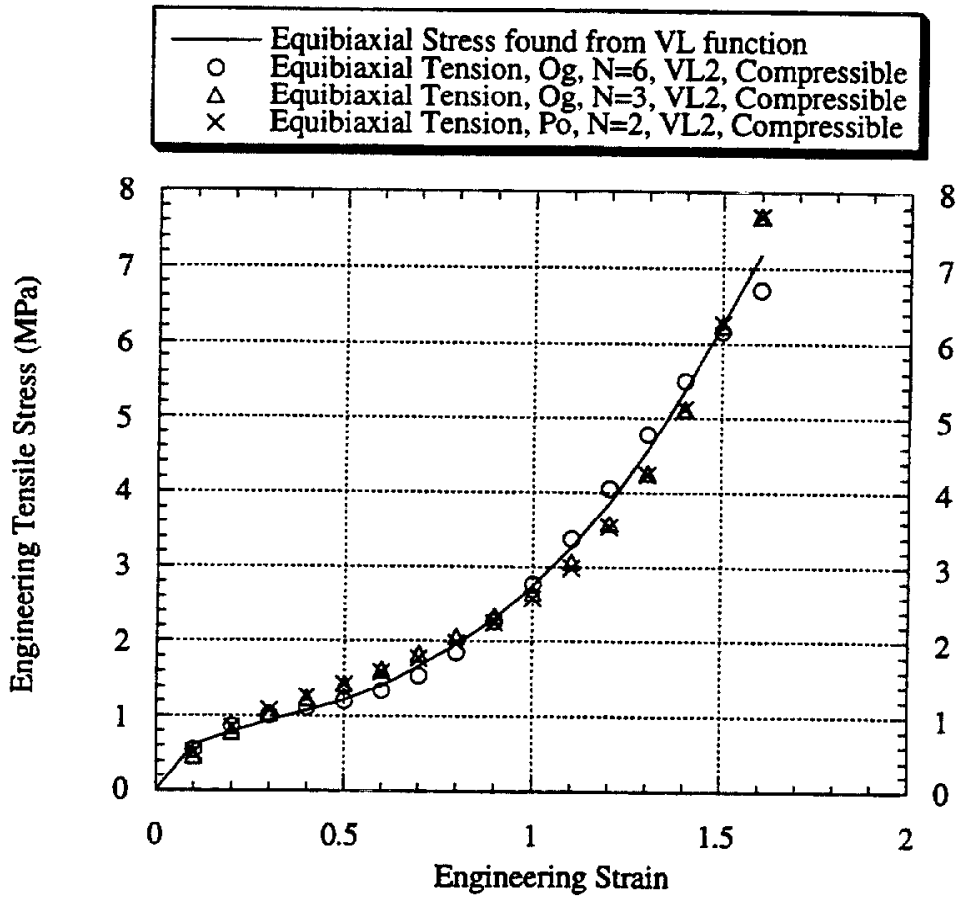

Figure 4.13 - Calibration to equibiaxial tension with compressibility using VL2 


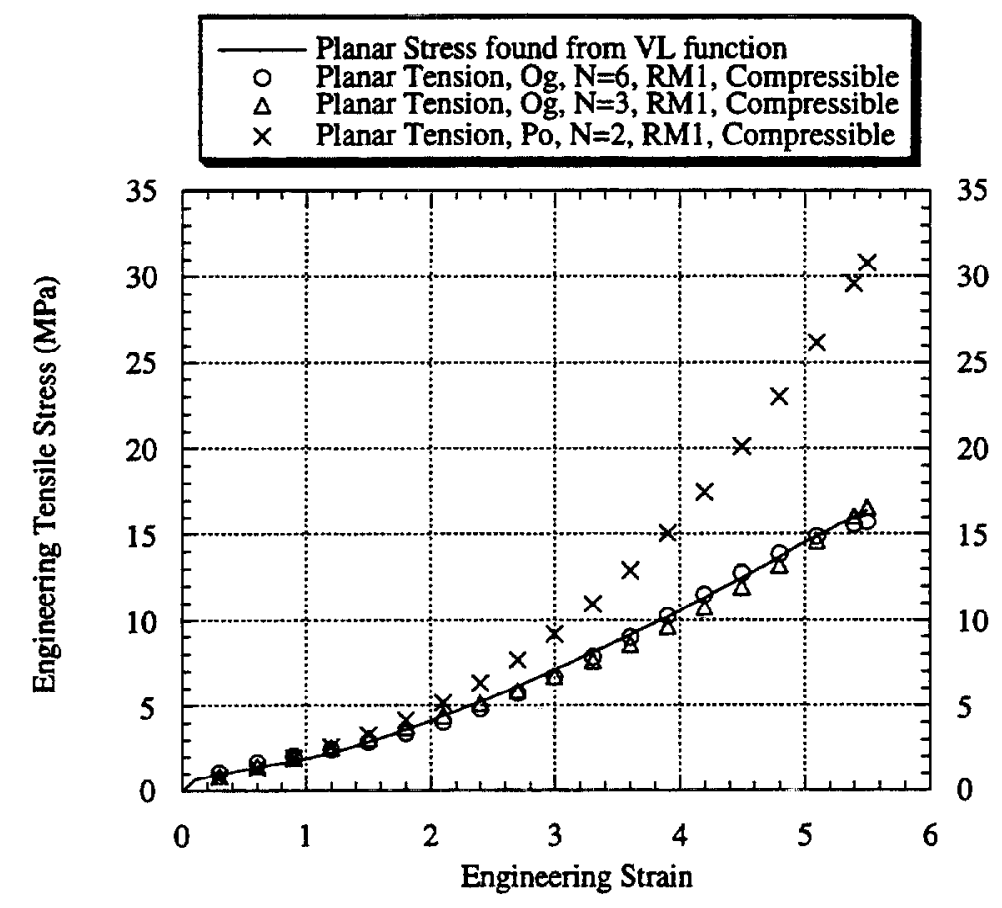

Figure 4.14 - Calibration to planar tension with compressibility using RM1

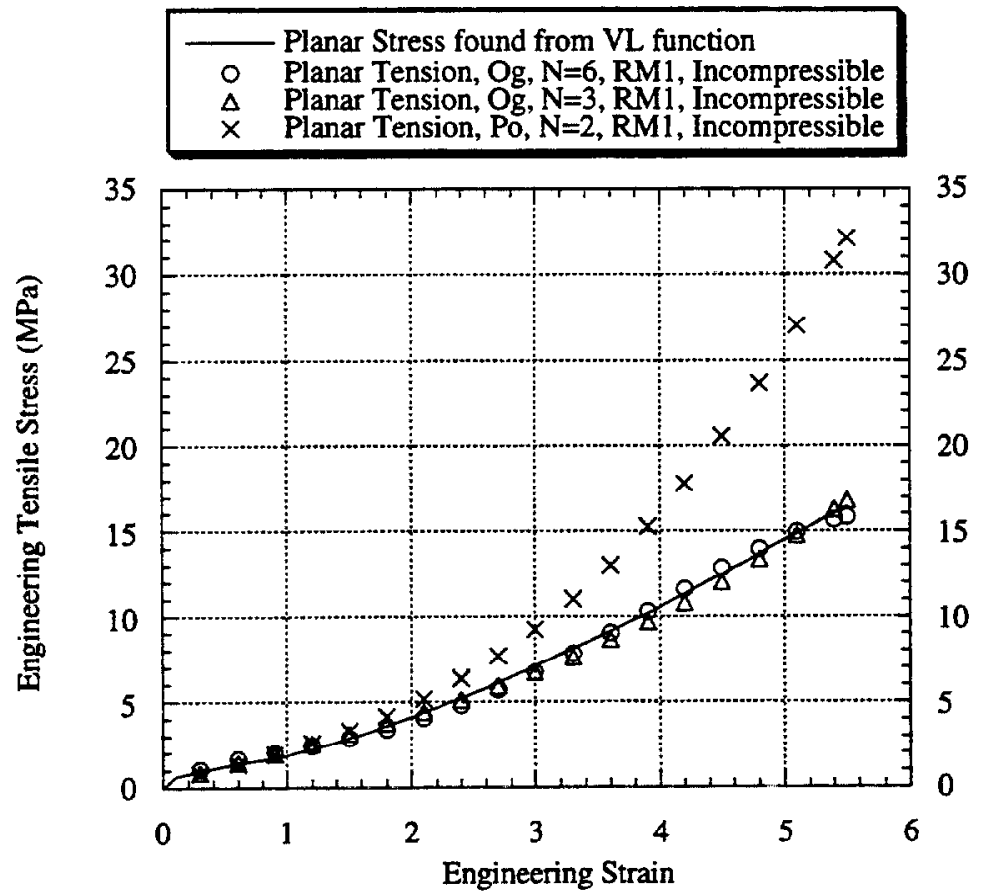

Figure 4.15 - Calibration to planar tension without compressibility using RM1 


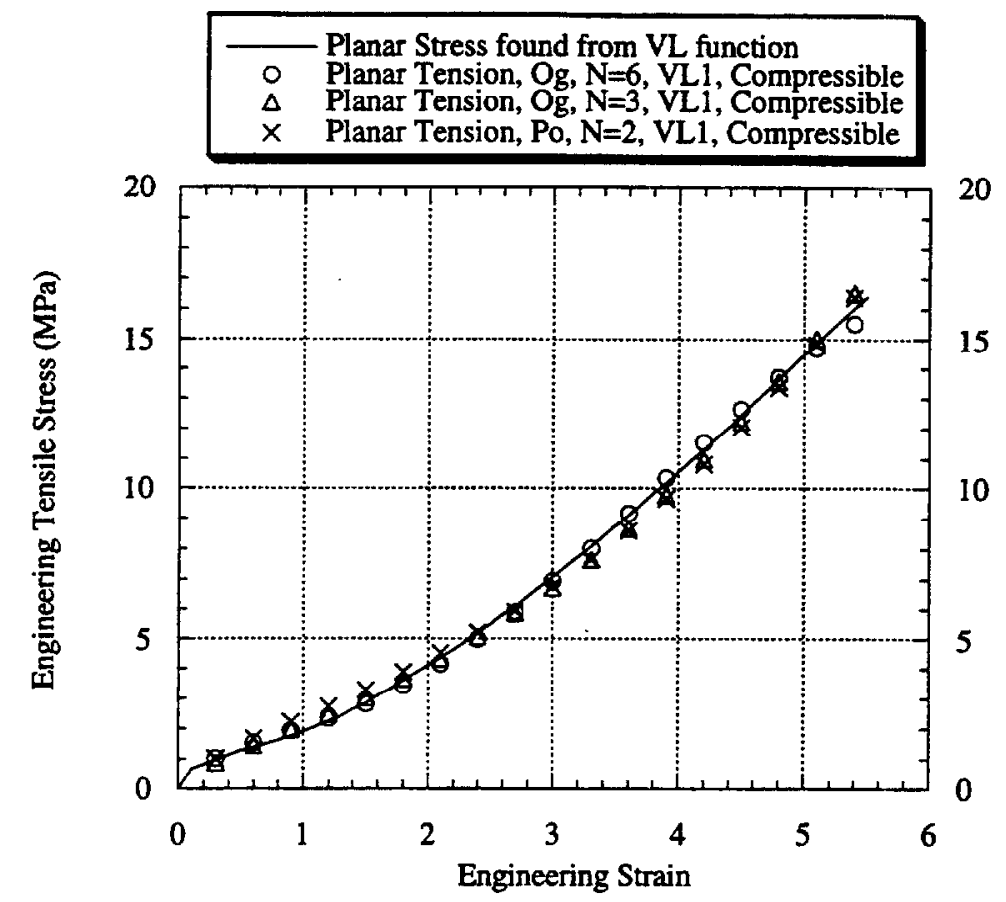

Figure 4.16 - Calibration to planar tension with compressibility using VL1

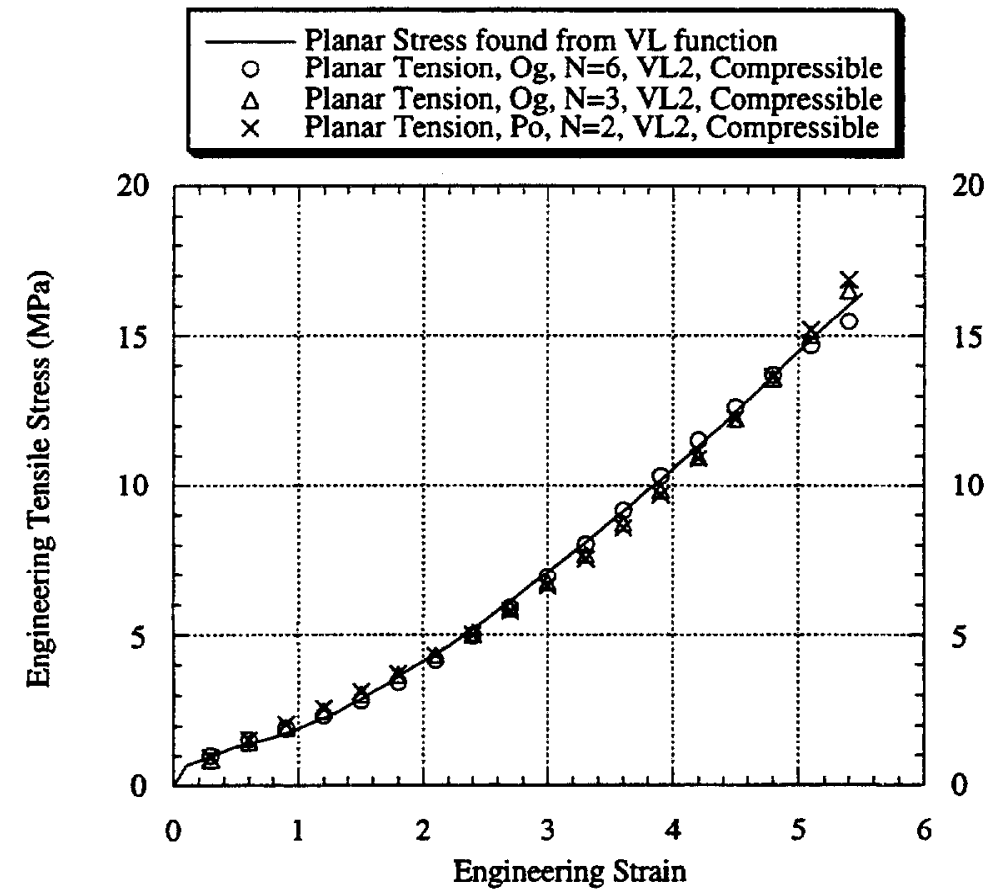

Figure 4.17 - Calibration to planar tension with compressibility using VL2 


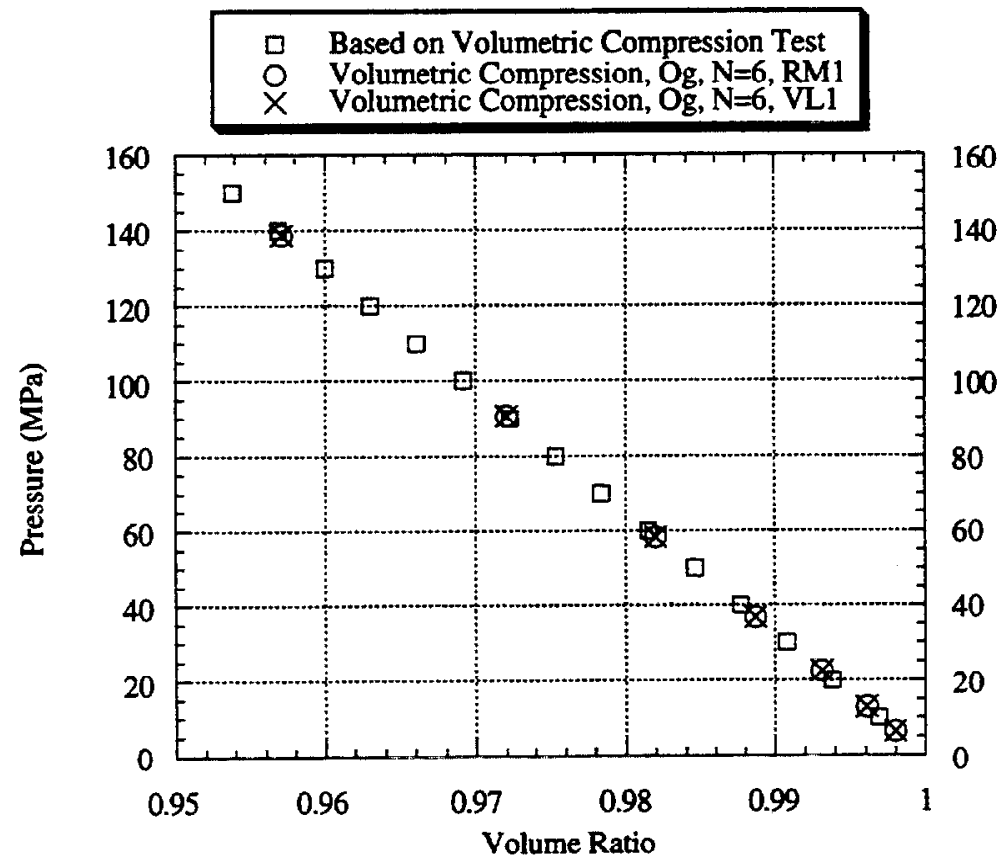

Figure 4.18 - Calibration to volumetric compression test using RM1 and VL1 models

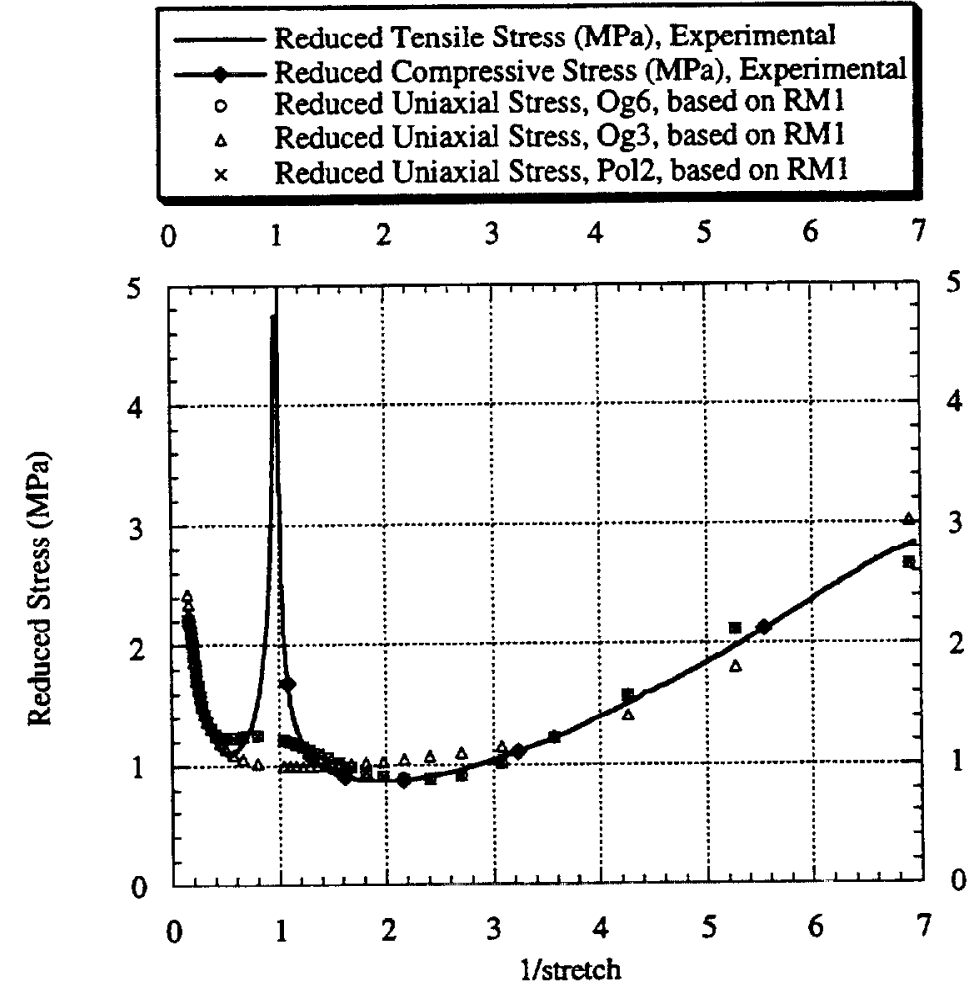

Figure 4.19 - Reduced uniaxial stresses from experiment vs ABAQUS results, using RM1 


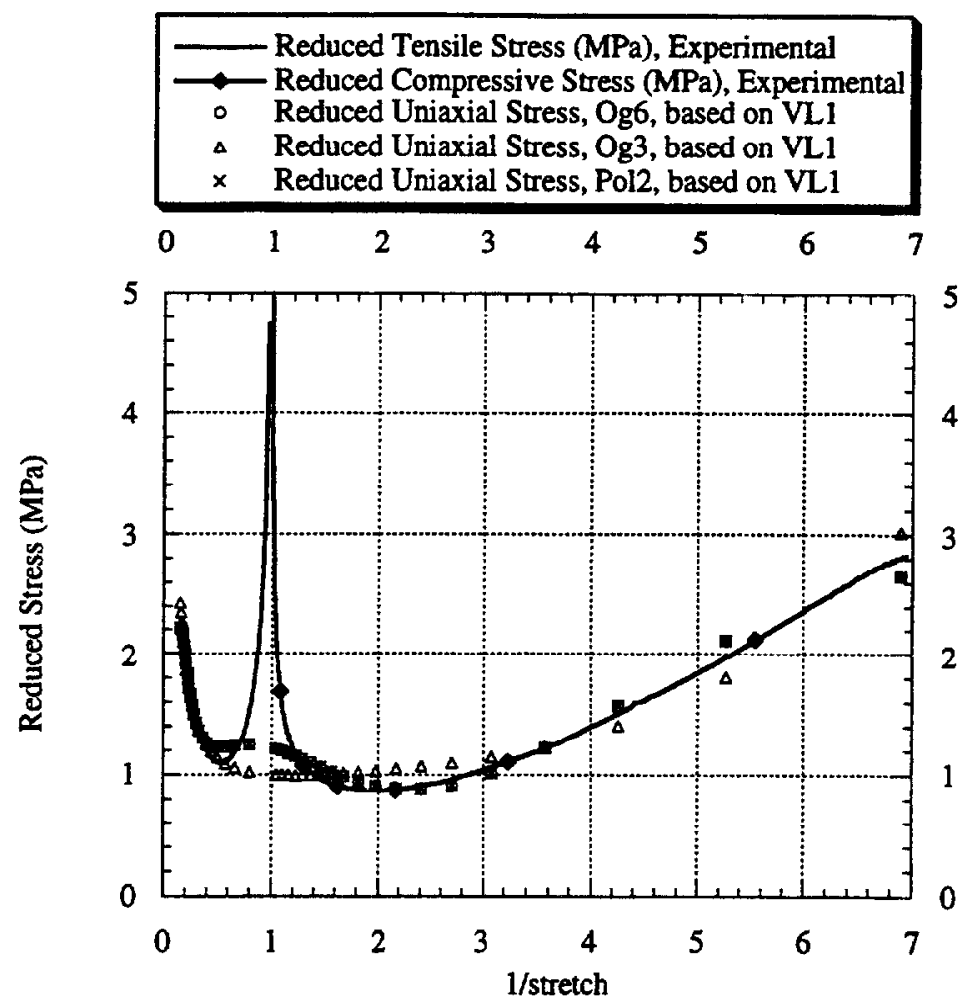

Figure 4.20 - Reduced uniaxial stresses from experiment vs ABAQUS results, using VL1 


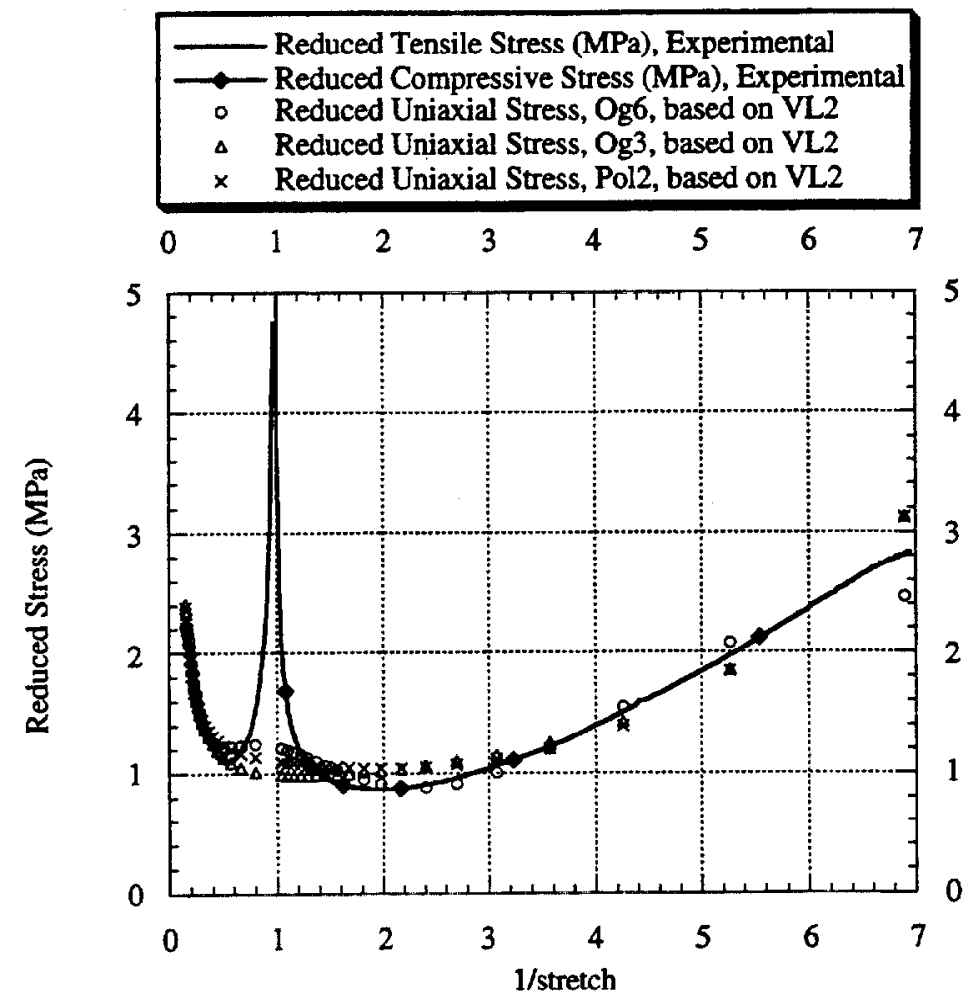

Figure 4.21 - Reduced uniaxial stresses from experiment vs. ABAQUS results, using VL2 


\section{BLANK PAGE}




\section{FINITE ELEMENT MODELING OF STEEL}

When compressively loading the bearings, the steel shims undergo plastic straining, and eventually some steel shims rupture, causing the bearing to lose loadcarrying capacity. Therefore, in order to predict the shape of the bearing compressive stress-strain curve into the failure region, the plastic deformation of the steel must be modeled. Uniaxial tensile tests on steel specimens have shown a linear elastic response followed by a strain-hardening plastic range. This type of response can be modeled in most finite element codes. In ABAQUS, the material's elastic behavior is defined by furnishing the Elastic Modulus and Poisson's ratio ( 0.3 in all runs) under the ELASTIC option. The plastic data are defined in the PLASTIC option by giving the current yield stress as a function of the plastic strain.

Prior to shim failure, necking occurs. Necking is localized yielding in the steel. A triaxial state of stress exists during necking. The model used in this analysis cannot accurately predict the plastic flow at this point. Consequently, the steel model cannot predict failure of the shims. However, we are able to capture the gross plastic flow and therefore get an accurate depiction of the deformed shape of the bearing for much of the loading history.

\subsection{Steel Testing Methods}

For the B-Bearings, the shims were made of ASTM A570 Grade 36 steel. To prepare the shims for the bonding process with the rubber, they are sandblasted and then coated with a chemical compound in a process known as chemlocking. Tests were done on smooth coupons, sandblasted coupons, and chemlocked coupons in order to determine if sandblasting and chemlocking had a noticeable effect on the steel material properties. A change in behavior by sandblasting and/or chemlocking must be taken into account so that accurate predictions of full-scale bearing behavior can be made from scale model behavior. For instance, in sandblasting, fine grains of sand are propelled at the specimen under high pressure, giving the surface a pitted, uneven texture. The depth of this unevenness would be a higher percentage of the overall thickness for a thinner steel specimen, and may change its mechanical properties. Chemlock, on the other hand, is an adhesive that is painted on the sandblasted specimen in order to improve the steel/rubber bond during the vulcanization process. Since the chemlock is applied only on the surface of the steel, it appears unlikely that it would have any significant affect on the steel properties.

Five steel sheets were provided from which dumbbell specimens were cut, three specimens from each of the five steel sheets, for a total of fifteen tensile specimens, as shown in Table 5.1. 
Table 5.1 - Tensile dogbone specimens by finish

\begin{tabular}{cccc}
\hline Sheet Thickness & Finish & \# tensile specimens & $\begin{array}{c}\text { Specimen } \\
\text { Designation }\end{array}$ \\
\hline 12-gage & smooth & 3 & S\#12* \\
12-gage & sandblasted & 3 & SB\#12 \\
12-gage & chemlocked & 3 & $\mathrm{CL} \# 12$ \\
16-gage & sandblasted & 3 & $\mathrm{SB} \# 16$ \\
22-gage & sandblasted & 3 & $\mathrm{SB} \# 22$ \\
\hline
\end{tabular}

*\# refers to specimen 1,2, or 3 .

The tension tests were conducted in accordance with ASTM E 8-95a, Standard Test Methods for Tension Testing of Metallic Materials. Figure 5.1 shows the dimensions for the tensile specimens. The tolerance on the width of the reduced section is per the ASTM Standards, as is the minimum radius on the fillets. The ends of the specimen were cut at least 3.00 inches in length to ensure adequate gripping in our test machine.

The relevant stress and strain measures are true (Cauchy) stress and logarithmic strain for input into ABAQUS, and material data for all these models must be given in these measures. The testing machine automatically generated an engineering stress-strain curve for each test. Special steps were necessary to find the true stress and logarithmic strain, and are described below.

During plastic straining and prior to necking, it is well established that a metal maintains its volume, i.e. it is essentially incompressible (Dowling, 1993; Dieter, 1976). This observation allows a simple transformation from engineering stress $(\sigma)$ to true stress $(t)$. Since the material is incompressible, $A l=A_{o} l_{o} \Rightarrow \frac{A_{o}}{A}=\frac{l}{l_{o}}$, where $l$ is the current gage length, $l_{o}$ is the original gage length, and $A_{o}$ is the original crosssectional area. Therefore,

$$
t=\frac{F}{A}=\frac{F}{A_{o}} \cdot \frac{A_{o}}{A}=\sigma \cdot \frac{A_{o}}{A}=\sigma \cdot \frac{l}{l_{o}}=\sigma(1+e)
$$

where $e$ is engineering strain.

Also, the logarithmic strain $\varepsilon$ is defined as $\varepsilon=\int_{l_{o}}^{l} \frac{d l}{l}$, or 


$$
\tilde{\varepsilon}=\ln \left(\frac{l}{l_{o}}\right)=\ln (1+e)
$$

Strictly speaking, Equation (5.1) only applies in the plastic range up to necking. This equation was applied to the elastic region also, since the error in doing so is no larger than $e$, which is 0.002 at yielding. The correct true stress falls between the engineering stress and the true stress as given by Equation (5.1), so $0.2 \%$ is an upper bound on the error.

Once necking starts, nearly all of the strain is localized at the neck. Since Equations (5.1) and (5.2) represent an average strain across the gage length, they are not valid. Actual measurement of the cross-sectional area at the point of necking must be made, and $t=\frac{F}{A}$ and $\varepsilon=\ln \left(\frac{A_{o}}{A}\right)$.

The width of the neck was measured using a caliper with a digital readout accurate to $0.01 \mathrm{~mm}$. Three micrometers were used to measure the thickness of the specimens prior to loading. Figure 5.2 shows the micrometer designs.

Micrometer \#1, with circular anvils, had a vernier which allowed measurements to an accuracy of 0.0001 in. Micrometers \#2 and \#3 were accurate to 0.001 in. Micrometer \#1 was the most accurate instrument, and the greatest confidence was placed in its measurements. Each micrometer was used to measure the original thickness in order to ensure that measurements between them correlated well, which they did. However, the circular anvils of Micrometer \#1 were too large to measure the thickness of the specimens in the necking region. This was also true for Micrometer \#2. Micrometer \#3's anvils came to a point, allowing more accurate measurements in the necking region. Therefore, Micrometer $\# 3$ was used to measure the thickness in the necking region.

The thickness measurement in the necking region was tricky. First, at the onset of necking, which occurs at the maximum force F (Dowling, 1993; Dieter, 1976), necking was not visible. Once the load started to drop, the test machine was stopped at intervals, and the necking measurements were made. It was noticed that the location where minimum measurements were recorded shortly after the maximum force $F$ was reached (in which necking was not visible) did in fact correspond to the location where necking became visible shortly thereafter. Therefore, once localized straining occurred, it didn't shift to another location, so the location of the initial minimum measurements corresponded with the location of the later minimum measurements. Second, the neck wasn't perpendicular to the longitudinal axis. If the failure mechanism is shearing, or sliding of the molecules, the maximum shear should be at a $45^{\circ}$ angle to the longitudinal axis, which is the axis of loading. In practice, this angle varied between $90^{\circ}$ and $45^{\circ}$, sometimes fairly close to $90^{\circ}$, but in general closer to $45^{\circ}$. Care had to be taken to make the measurements along this line. 


\subsection{Steel Testing Results}

Figures 5.3 through 5.7 show the results of each of the five sets of tests. Necking begins when each of the solid lines ends. The stress-strain points in this region are based on actual measurements of thickness in the necking region. The shape of the curve in the necking region is generally linear, which is in close. agreement with true stress-strain curves shown in Dowling (1993) and Dieter (1976). There is some variation, and this is primarily due to inaccurate recording of the load at which the test machine was stopped (in order to take the thickness measurements) and inaccurate thickness measurements. These variations from linearity are more prevalent at higher strains, due in some cases to the fact that the load was rapidly declining at higher strains, leading to inaccurate recording of load.

The repeatability of each set of tests was good. The stresses for each of the three curves for each sample differ by less than $1.5 \%$ in the plastic range, with the exception of the 22-gage tests, where the stresses for SB3-22 are about $7 \%$ larger than SB1-22. There are only two specimens in the SB\#-12 series (one specimen in the series was damaged during testing).

Figure 5.8 is a comparison by finish of the three 12-gage samples, which demonstrates that the stress for the chemlocked specimens was significantly lower in the plastic region than for the smooth or sandblasted specimens. We requested the bearing manufacturer to supply us with steel samples from the same millings used in the manufacture of the shims. However, we cannot be sure that the samples received were from the same millings. Differences in steel behavior may be due to the steel coming from different steel millings. It is common to see yield stresses between 248 $\mathrm{MPa}(36 \mathrm{ksi})$ and $345 \mathrm{MPa}(50 \mathrm{ksi})$ from milling to milling for the steel used in the $\mathrm{B}$ Bearings, ASTM A570 Grade 36 steel. Nevertheless, in our samples, the yield stresses between the 12-gage samples were $260 \mathrm{MPa}$ for the sandblasted and chemlocked specimens versus $270 \mathrm{MPa}$ for the smooth specimens, a difference of only about $3.8 \%$. The larger difference in behavior shows up in the plastic range, where the stresses, for strains larger than 0.2 , are about $13 \%$ lower for the chemlocked specimen compared to the sandblasted and smooth specimens.

Figure 5.9 is a comparison between the 12,16 , and 22-gage specimens, and shows that the stress for CL1-12, over the plastic range of 0.2 to 1.0 , is within $1.0 \%$ of SB1-16 and no more than 6.0\% less than SB2-22.

\subsection{Measurement Accuracy and Variability and Effect on Bearing Response}

A Satec tension and compression machine was used to test the steel specimens. The accuracy of this machine is established through a calibration using known dead loads, and was calibrated according to ASTM E4, which requires that the machine reading be within $\pm 1 \%$ of the true reading. The machine has met these requirements. This is equivalent to a relative expanded uncertainty $(\mathrm{k}=2)$ of $1 \%$, and is small compared to specimen variability.

Table 5.2 summarizes the testing results for the elastic properties. Note that the elastic moduli are about the same for each type of specimen, but the yield stress is about $10 \%$ higher for the 22 -gage specimen. 
Table 5.2 - Comparison of elastic properties for steel specimens

\begin{tabular}{lcc}
\hline \multicolumn{1}{c}{ Steel Specimen } & $\begin{array}{c}\text { Yield Stress } \\
\text { (MPa) }\end{array}$ & $\begin{array}{c}\text { Elastic Modulus } \\
\text { (E) }\end{array}$ \\
\hline 12-gage Smooth Finish (S3-12) & 270 & $2.1 \times 10^{5}$ \\
12-gage Sandblasted Finish (SB2-12) & 262 & $2.1 \times 10^{5}$ \\
12-gage Chemlocked Finish (CL1-12) & 262 & $2.2 \times 10^{5}$ \\
16-gage Sandblasted Finish (SB1-16) & 261 & $2.3 \times 10^{5}$ \\
22-gage Sandblasted Finish (SB2-22) & 290 & $2.0 \times 10^{5}$ \\
\hline
\end{tabular}

The test results for each specimen in the plastic range fall into two distinct groups in which the stress-strain responses are similar. In the first group, consisting of the 12-gage smooth and sandblasted specimens, the stresses are more than $10 \%$ higher than the stresses in the second group, consisting of the 12-gage chemlocked specimen, the 16-gage chemlocked specimen, and the 22-gage sandblasted specimen (c.f. Figures 5.8 and 5.9). Figure 5.10 compares the second group more closely, up to a logarithmic strain of 0.60 , and shows that the stresses of the 22-gage sandblasted shim are approximately $4 \%$ to $6 \%$ higher than the stresses of the 12 -gage chemlocked specimen and the 16-gage sandblasted specimen. The differences in these samples indicate differences in material properties, since the variations are larger than the resolution of the testing machine.

Based on these testing results, we expect similar bearing behavior predicted by the finite element analysis up to initial shim yielding when incorporating any of the 12-gage samples or the 16-gage sample in the model, but higher stresses at initial shim yielding when incorporating the 22-gage sample (assuming usage of the same rubber model and model geometry for all runs). From initial shim yielding to compressive strains near bearing failure, the steel is yielding, and we expect the predicted response from the finite element analysis to be virtually the same with inclusion of either the 12-gage chemlocked sampleor the 16-gage sandblasted sample, and approximately $4 \%$ to $6 \%$ higher for the 22-gage sandblasted sample (c.f. Figures 5.9 and 5.10). We

- expect the stresses in the plastic range to be significantly higher than the foregoing with inclusion of either the 12-gage smooth or sandblasted specimens in the model (c.f. Figure 5.8). Finite element analysis of the bearings, discussed in detail in Section 6 , verify the validity of the expected responses. 


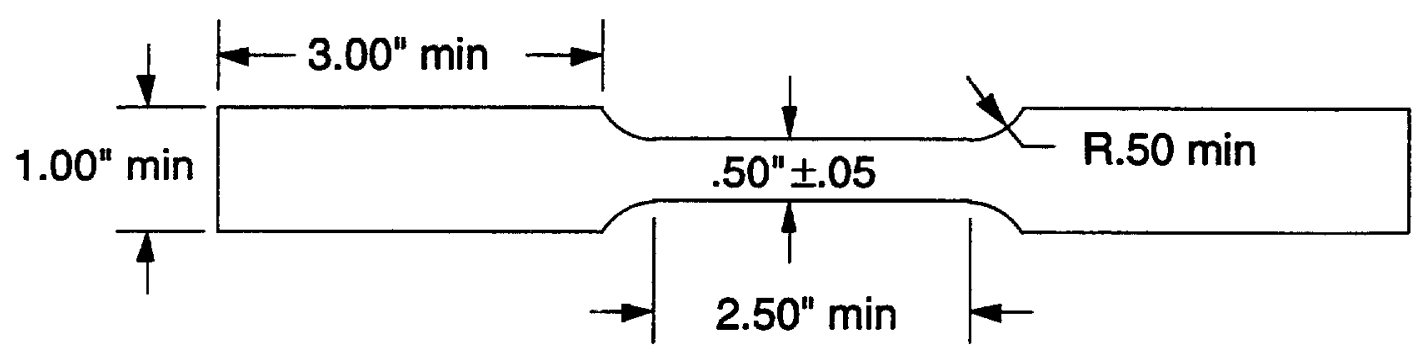

Figure 5.1 - Dimensions of tensile dogbone specimens

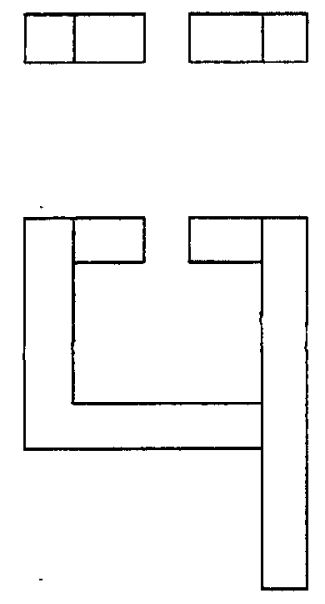

Micrometer \#1
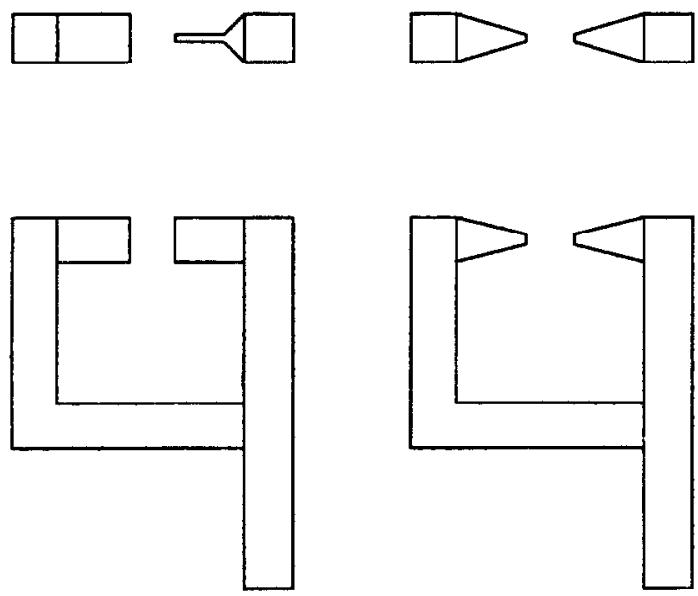

Micrometer \#2
FRONT VIEW

Micrometer \#3

Figure 5.2 - Micrometers used to measure the necking region of the tensile dogbone specimens 


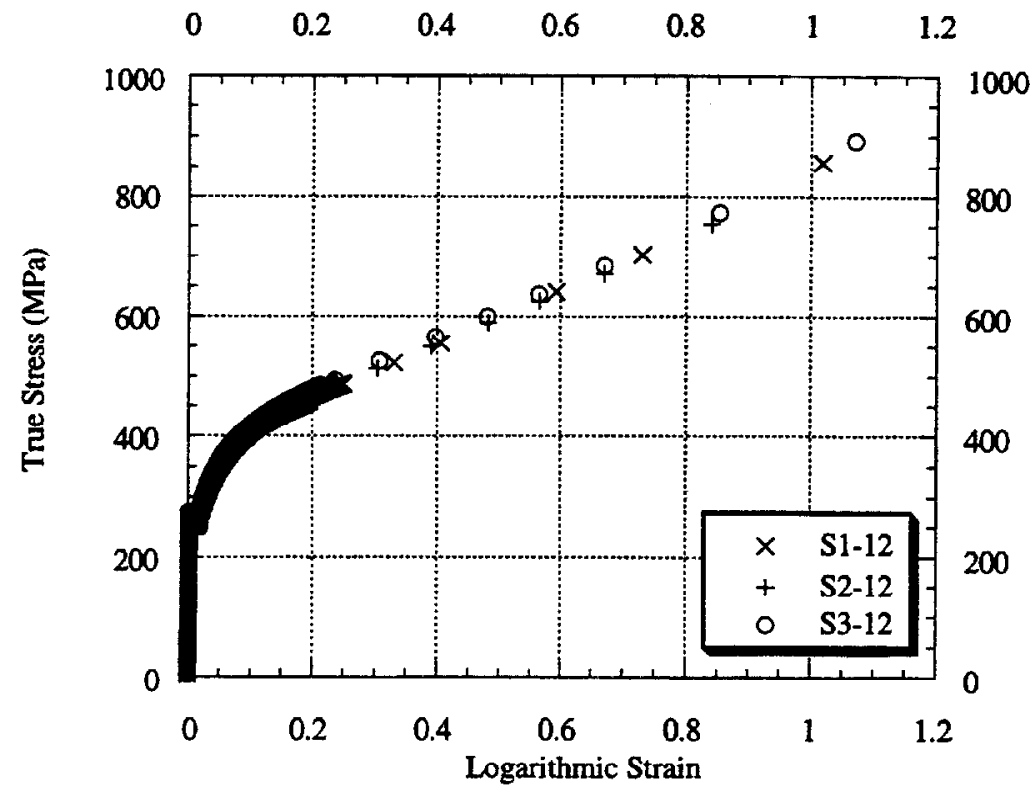

Figure 5.3 - True stress-strain curves for smooth (S) 12-gage specimens

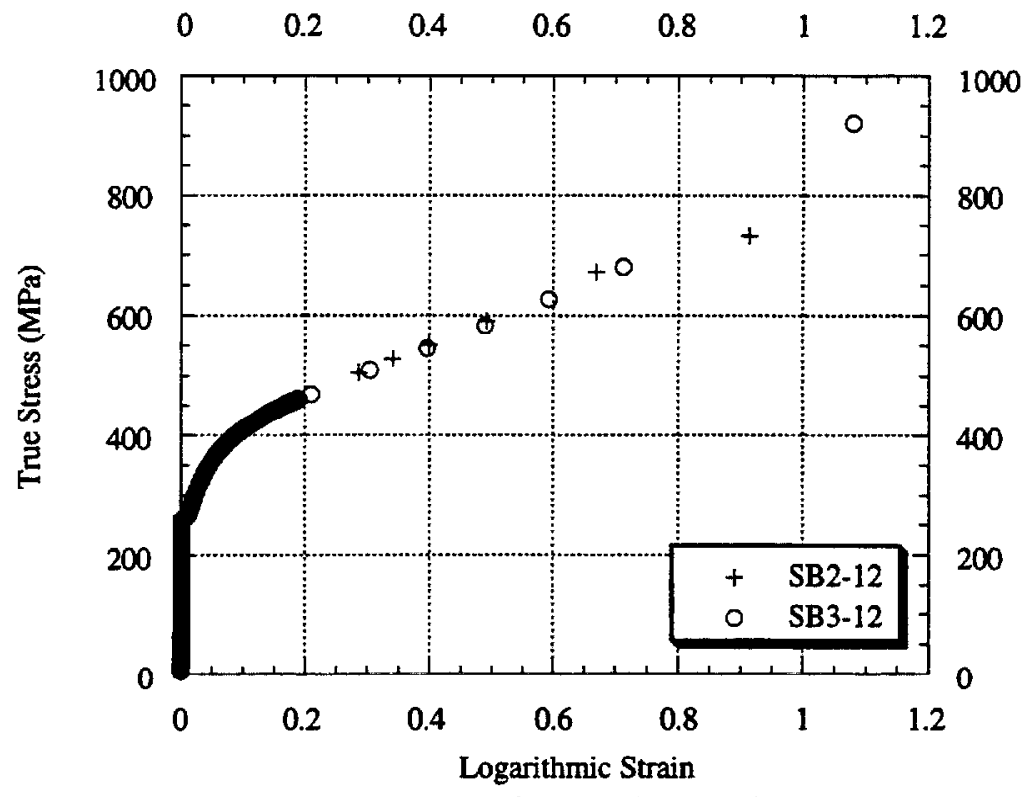

Figure 5.4 - True stress-strain curves for sandblasted (SB) 12-gage specimens 


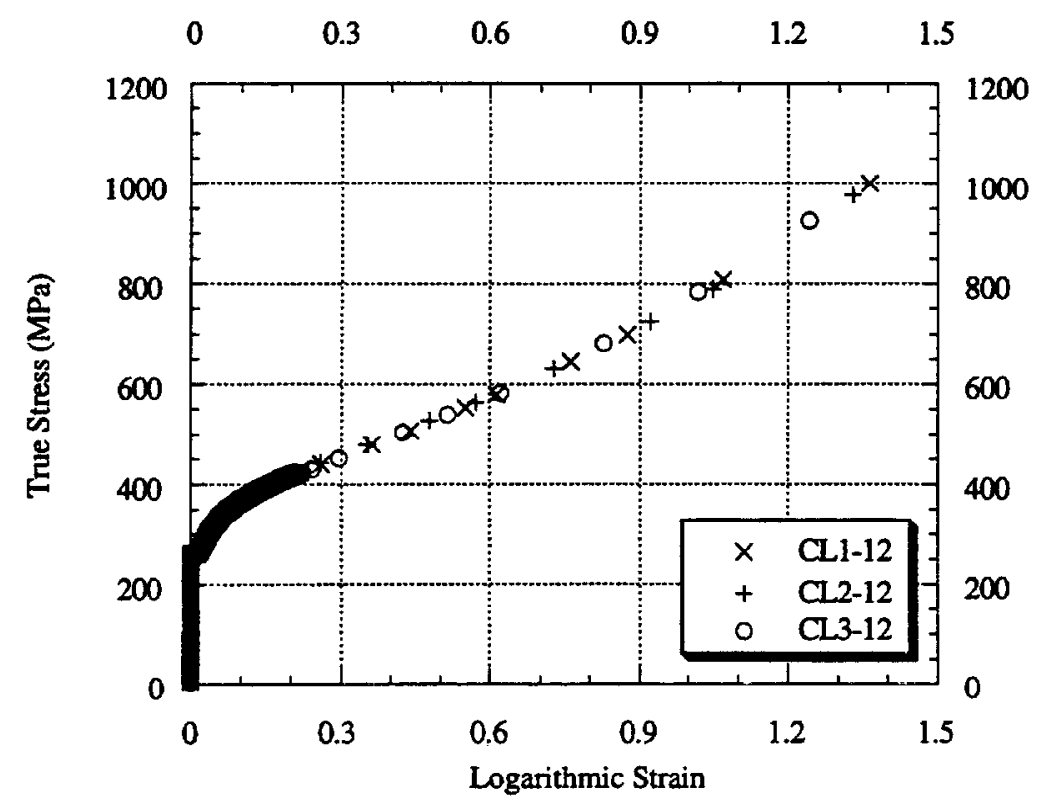

Figure 5.5 - True stress-strain curves for chemlocked (CL) 12-gage specimens

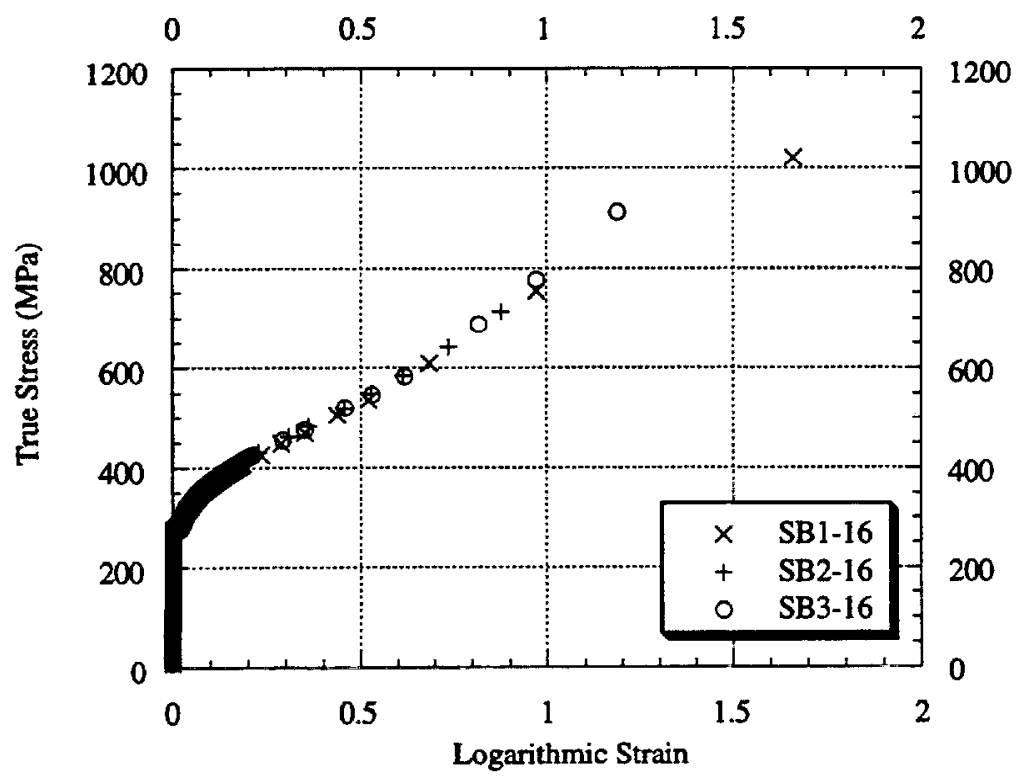

Figure 5.6 - True stress-strain curves for sandblasted (SB) 16-gage specimens 


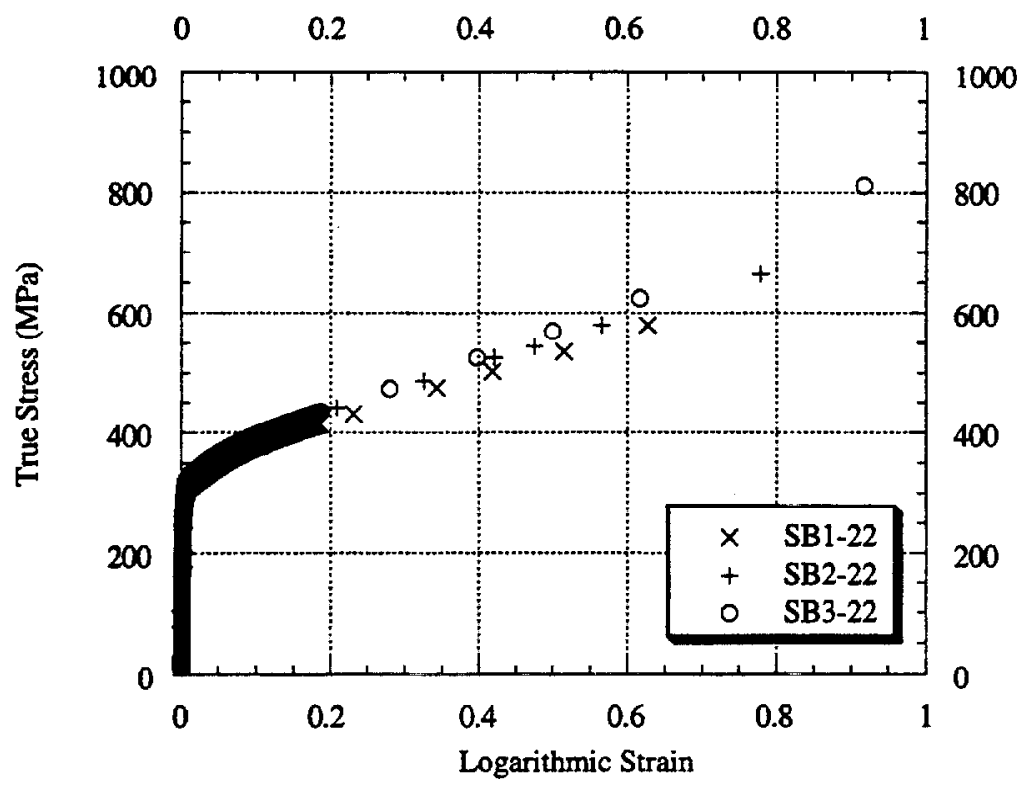

Figure 5.7 - True stress-strain Curves for sandblasted (SB) 22-gage specimens by finish

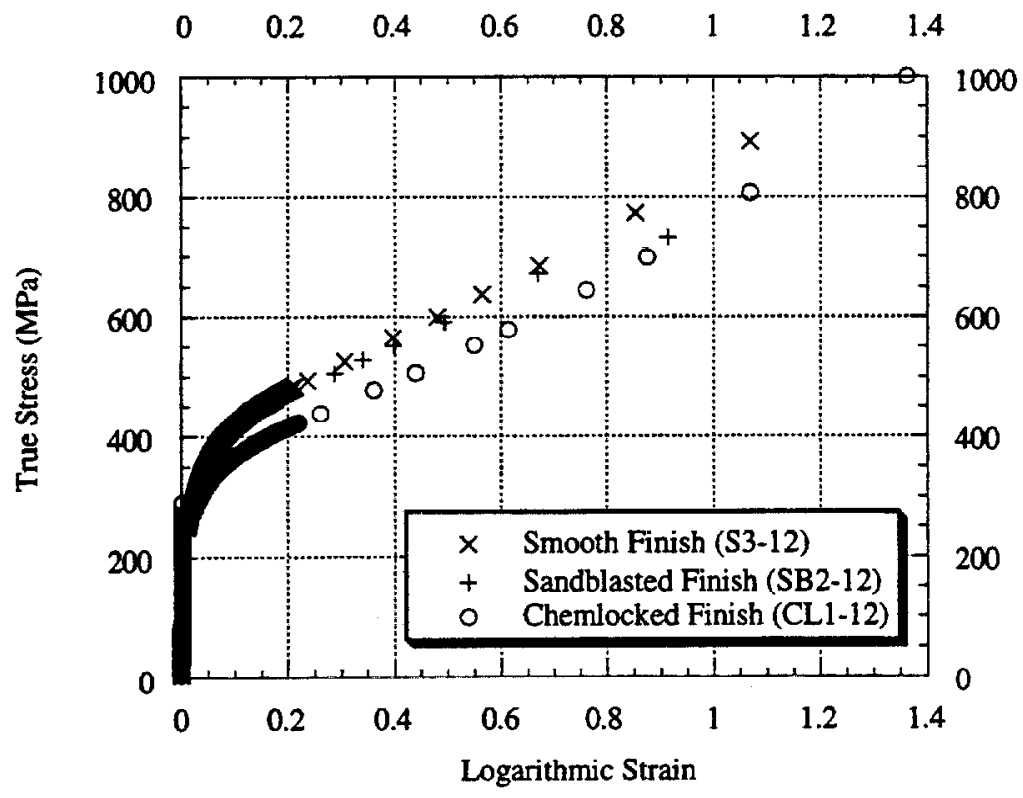

Figure 5.8 - Comparison of true stress-strain curves for 12-gage steel samples 


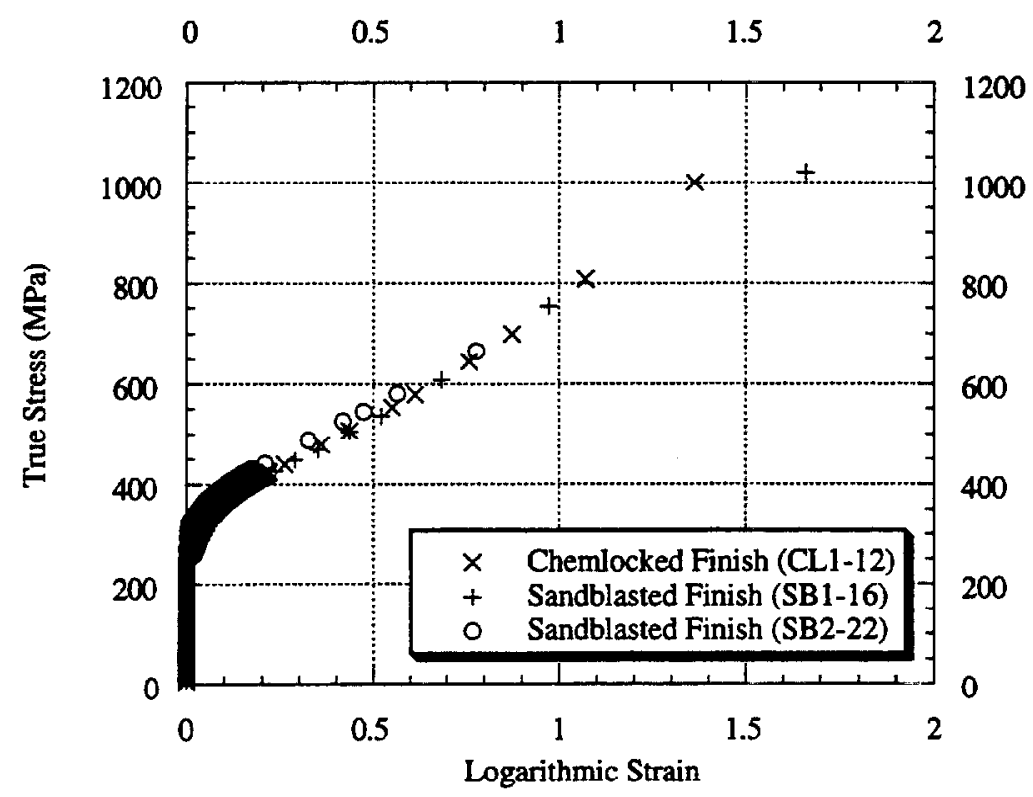

Figure 5.9 - Comparison of 12,16, and 22-gage steel samples

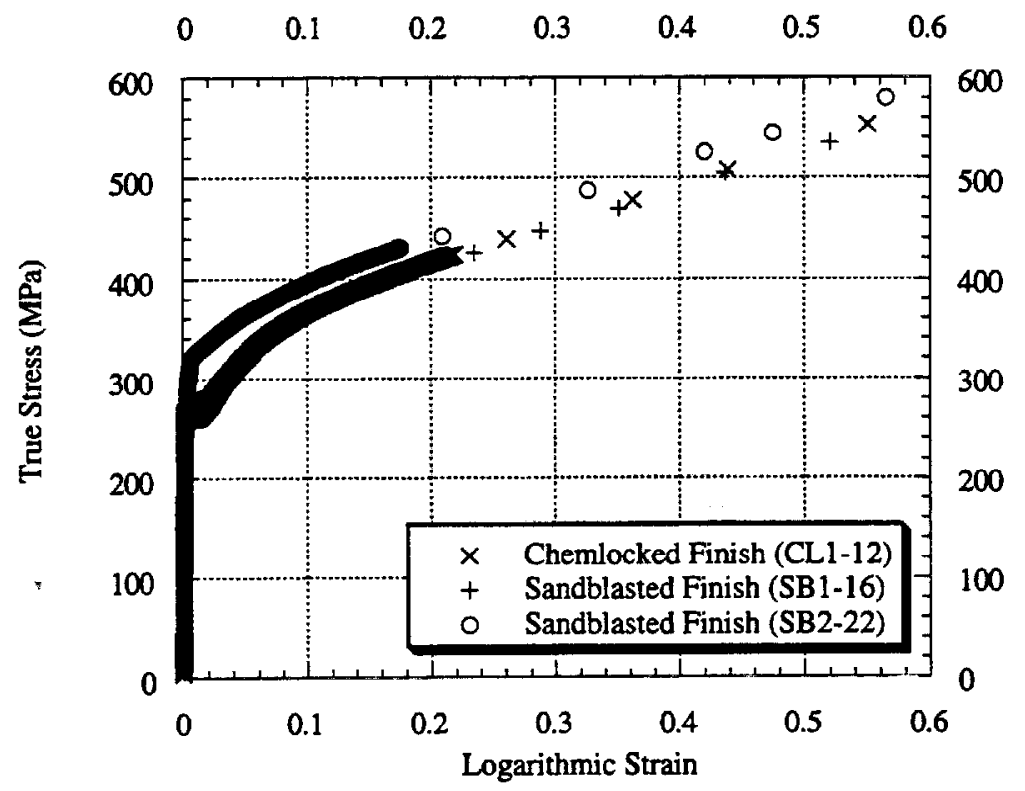

Figure 5.10 - Comparison of 12,16, and 22-gage steel samples up to a logarithmic strain of 0.60 


\section{FINITE ELEMENT MODELING OF THE ELASTOMERIC BEARINGS}

\subsection{Discretization of the Finite Element Models of the Bearings}

As noted in Section 5, the finite element model does not have the capability to model failure of the steel shims, since the plastic flow of the steel in the necking region cannot be accurately modeled. This is not a drawback in the context of this research, because we are primarily interested in predicting the ultimate capacity of a full-scale bearing based on a test to failure of a scale model bearing. Comparison of stress-strain curves between scale models and prototypes will be the primary means of determining whether the failure stress of a scale model is representative of a full-scale bearing. The results of scale model analyses are discussed in Section 6.5.

Assuming the load to be uniformly distributed over the bearings, an axisymmetric model was used. There is also symmetry between the top and bottom half of the bearing. Therefore, only $1 / 4$ of the bearing was modeled. The model represents a slice of the bearing through the $R-Z$ plane, from $R=0$ to the outer edge, and from the middle to the top.

Each rubber layer was modeled with four layers of 2-dimensional linear displacement continuum axisymmetric hybrid elements with full integration (CAX4H), and each steel shim was modeled with CAX4 elements, the same as for rubber except the hybrid feature is not used. Since the rubber at the cover makes contact with the endplate in the course of the loading history, the endplate and cover were modeled using the ABAQUS CONTACT PAIR option with the endplate as the master surface and the outside surfaces of certain elements in the cover as the slave surface. Since deformations of the endplate are negligible, it was modeled using the 1 -dimensional rigid elements RAX2. The static coefficient of friction between the rubber and end plate is $\mu=0.5$, based on a simple test which determined the critical friction angle as $45^{\circ}$. Figures 6.1 through 6.3 show the undeformed meshes for the Full-Scale, 1/2-Scale, and 1/4-Scale models, respectively. All deformed mesh and contour plots were generated in PATRAN. The X-Y plane in these PATRAN generated plots corresponds to the R-Z plane of the axisymmetric model.

\subsection{Effect of Different Factors on the Results of the Finite Element Model}

\subsubsection{Inclusion of the Central Core in the Model}

After testing to failure, the B-Bearings were cut open. Figure 6.4 is a photograph of a vertical slice through the core of Bearing B2, the 1/2-Scale Bearing. This photograph provides a baseline on which to judge the accuracy of the deformed shapes generated by the finite element analysis.

As noted earlier, the core of the bearing is filled with rubber after stacking of the rubber and shim layers. We attempted to model the bearing with the core. The ABAQUS filename for this model is procore_RM1_CS7_CL112:1, which means Prototype with the core using the RM1 rubber model, volumetric data CS7, and steel data CL1-12. However, the analysis failed to converge beyond a bearing compressive strain of $14.3 \%$, which is $54 \%$ of the strain when the actual bearing failed. Figure 6.5 shows the deformation in the rubber and steel at this point. We believe the analytical problem is due to excessive distortion of the rubber elements in the core that are 
adjacent to the steel elements (all elements were initially rectangular in shape). In the actual bearing, the stresses in the core rubber at the interface with the bonded portion of the bearing are so great that the core rubber splits away from the rubber and steel in this region. This would have the affect of reducing the stresses in this region. This loss of contact has not been modeled.

By excluding the core, we were able to get the analyses to go out beyond the bearing compressive strains at which bearing failure occured (finite element run entitled "pro_RM1_CS7_CL112:1"). Figure 6.6 shows the ABAQUS runs with and without the core in comparison with the experimental Prototype Bearing stress-strain curve. Based on this result, it is reasonable to assume that the rubber core has negligible effect on the behavior of the bearing. Furthermore, the finite element model performs well in estimating the experimental curve, with the exception that the finite element model is stiffer prior to yielding of the shims. The abrupt change in modulus of the bearing stress-strain curve is due to yielding of the shims. For the experiment on the Prototype, this occured at approximately $42 \mathrm{MPa}$, versus about $40 \mathrm{MPa}$ for the finite element run pro_RM1_CS7_CL112:1. A secant modulus at the point of initial shim yielding gives $667 \mathrm{MPa}$ for the experimental test versus $1025 \mathrm{MPa}$ for the finite element run pro_RM1_CS7_CL112:1. A straight line drawn from the shim yielding point to failure gives $235 \mathrm{MPa}$ for the experimental test versus $215 \mathrm{MPa}$ for the finite element run pro_RM1_CS7_CL112:1. Failure for the bearing occurs when a shim ruptures, which decreases the ability of the bearing to sustain load, and appears on the bearing stress-strain curve as a sudden drop at a bearing engineering compressive stress of $90 \mathrm{MPa}$ and strain of $0.267 \mathrm{~m} / \mathrm{m}$. The finite element run pro_RM1_CS7_CL112:1 gives a stress of $88.9 \mathrm{MPa}$ at a strain of $0.267 \mathrm{~m} / \mathrm{m}$, or $1.2 \%$ lower than experiment. Finite element runs with CL1-12 will be used as the baseline for comparisons between models.

\subsubsection{Comparison between the Ogden and Polynomial Strain Energy Functions and Rubber Models RM1 and VL1}

The finite element runs plotted in Figure 6.6, procore_RM1_CS7_CL112:1 and pro_RM1_CS7_CL112:1, were conducted using the Ogden Strain Energy Potential with $\mathrm{N}=6$. Figure 6.7 is a comparison of pro_RM1_CS7_CL112:1 with a finite element run using the Polynomial Strain Energy Function, $\bar{N}=2$ (filename pro_RM1_CS7_CL112_Po2:1). The results are virtually identical. All other runs discussed in the following sections use the Ogden Strain Energy Potential with $N=6$.

Figure 6.8 shows the engineering stress-strain curve for the Prototype Bearing, along with the finite element results using rubber models RM1 and VL1, the volumetric data from CompSet 7 (CS7), and steel data CL1-12. Both models give the same response. We are tempted to attribute this to the fact that the finite element program, using RM1 (which does not include the planar tensile stresses), accurately estimates the planar stresses found from the Valanis-Landel function, and that are included in VL1. In other words, RM1 and VL1 are virtually identical. However, these rubber models only contribute to the deviatoric portion of the strain energy function. To reinforce this statement, the Ogden Strain Energy Potential is rewritten hère.

$$
U=\sum_{i=1}^{N} \frac{2 \mu_{i}}{\alpha_{i}^{2}}\left(\bar{\lambda}_{1}^{\alpha_{i}}+\bar{\lambda}_{2}^{\alpha_{i}}+\bar{\lambda}_{3}^{\alpha_{i}}-3\right)+\sum_{i=1}^{N} \frac{1}{D_{i}}\left(J_{e l}-1\right)^{2 i}
$$


The first summation of the strain energy potential is the deviatoric strain energy. Rubber models RM1 and VL1 are used to find only the parameters $\mu_{i}$ and $\alpha_{i}$, which define the deviatoric strain energy. The parameter $D_{i}$ is found from the volumetric test data (CS7), and determines the volumetric strain energy. Both models use CS7 to determine the volumetric strain energy.

As shown in Section 6.3, the hydrostatic stresses are two orders of magnitude greater than the deviatoric stresses, so that if RM1 and VL1 had yielded different deviatoric stresses, the compressive response of the bearing would have been affected very little.

\subsubsection{Rubber Compressibility}

The next two figures, Figures 6.9 and 6.10 , look at the effect of compressibility on the results. An incompressible rubber model makes the bearing much stiffer prior to yielding of the shims. The initial compressive modulus for the bearing modeled with the incompressible rubber assumption (pro_RM1_Incomp_CL112:1) is 1745 $\mathrm{MPa}$, which is $70 \%$ larger (1025 MPa) than the initial compressive modulus of the analysis done which included compressibility of the rubber (pro_RM1_CS7_CL112:1). Beyond initial shim yielding, although the predicted stresses are higher in the incompressible model, the stiffness, based on the slope of a straight line drawn from the point of initial shim yielding to the strain at which the bearing failed, is virtually the same (214 $\mathrm{MPa}$ for the Incompressible model versus $215 \mathrm{MPa}$ for the Compressible model). For the incompressible model, the stress at the strain at which the bearing failed is $90.3 \mathrm{MPa}$, which is only $0.3 \%$ higher than the stress at which the actual bearing failed ( $90 \mathrm{MPa})$, and only $1 \%$ higher than the failure stress in the compressible model $(88.9 \mathrm{MPa})$. Therefore, the inclusion of compressibility in the rubber model has a large effect on the compressive stiffness of the bearing prior to initial shim yielding, but has a small effect on the predicted stress at failure.

\subsubsection{Steel Test Data}

Figure 6.11 compares the finite element model using the chemlocked steel data versus the finite element model using the sandblasted steel data. The response for the bearing model based on steel specimen SB2-12 is stiffer in the plastic range compared to steel specimen CL1-12, and this is due to the difference in the tensile specimen stress-strain response of these steel models in the plastic range, as shown in Figure 6.12. Using specimen SB2-12, the bearing engineering compressive stress at the strain at which the bearing failed $(0.267 \mathrm{~m} / \mathrm{m})$ is $99.7 \mathrm{MPa}$, or $10.8 \%$ higher than experiment. Although not apparent from Figure 6.26, due to the large scale shown for the logarithmic strain, the yield stresses for the two steel models are nearly identical (261.9 MPa for steel specimen CL1-12 versus 261.6 MPa for steel specimen SB2-12). This is reflected in the identical shape of the bearing stress-strain curves up to initial yielding of the shims, as shown in Figure 6.11.

\subsection{Deformation and Contour Plots and the Dominance of Hydrostatic Stress}

Figure 6.13 is a plot of the finite element solution of the deformed shape of the rubber portion of the bearing for model pro_RM1_CS7_CL112:1 at Increment 96, which corresponds to a bearing engineering stress of $88.6 \mathrm{MPa}$ and a bearing engineering strain of $0.265 \mathrm{~m} / \mathrm{m}$. Consulting Figure 6.8 , this is very near the 
compressive engineering strain at which the Prototype failed. The deformed shape of the rubber is very similar to the actual deformed shape of the 1/2-Scale Bearing (Figure 6.4). Even though we are showing only the rubber elements here, the deformed shape of the shims is clearly visible between the rubber layers, and is also close to the actual deformed shape of the shims of the 1/2-Scale Bearing.

Superimposed on the bearing is the hydrostatic stress in the rubber, which reaches a maximum of $83.7 \mathrm{MPa}$. The hydrostatic stress is an invariant, and is the average of the three normal stresses. Figures 6.14 through 6.16 show the three principal stresses, each of which has a maximum value of about $83 \mathrm{MPa}$. Since the principal stresses have approximately the same magnitude, the maximum shearing stresses must be relatively small. Therefore, we conclude that for the loading condition modeled, the hydrostatic stresses are much larger than the deviatoric stresses.

The only plane in which shearing can occur is the $\mathrm{R}-\mathrm{Z}$ plane, due to symmetry. Figure 6.17 shows the maximum shearing stresses. For presentation of scalar data, which is shown in contour plots, PATRAN takes the largest and smallest values of the scalar variable, and divides the spectrum evenly. In Figure 6.17, the largest value of the maximum shear is $65.7 \mathrm{MPa}$, but this occurs only in a very few elements near the cover layer of the bearing. The great majority of the elements have a maximum shear stress between zero and $7.30 \mathrm{MPa}$. To better see the distribution of stresses in this lowest range of the spectrum, Figure 6.18 is shown, where the largest value was limited to 7.30 MPa. This figure clearly shows that the majority of the elements have a maximum shearing stress less than $1.46 \mathrm{MPa}$. Of course, any element in the highest spectrum range has a maximum shear value at least as large as that range, but this is a relatively small set of elements which are concentrated near the cover layer. The relatively low maximum shear values are not surprising, since rubber is weak in shear.

Figure 6.19 is the finite element solution of the deformed shape of the rubber portion of the bearing, along with the hydrostatic stresses, for the same model (pro_RM1_CS7_CL112:1) at Increment 8, which corresponds to a bearing engineering stress of $39.5 \mathrm{MPa}$ and a bearing engineering strain of $0.0383 \mathrm{~m} / \mathrm{m}$. This is the location on the engineering stress-strain curve where the slope changes abruptly, indicating yielding of the shims. At Increment 8, the hydrostatic stresses fall off more rapidly as we move toward the outer edge of the bearing as compared to Increment 96. The maximum value of the hydrostatic stress is $68.7 \mathrm{MPa}$, or about $82 \%$ of the maximum hydrostatic stress at Increment 96.

Figures 6.20 through 6.22 show the three principal stresses in the rubber at Increment 8 , and Figure 6.23 shows the maximum shearing stress in this same increment. Similar to Increment 96 , the hydrostatic stress is much larger than the maximum shearing stress; e.g., from the core to the middle of the bearing, the hydrostatic stress is between 53.0 and $68.7 \mathrm{MPa}$, and the maximum shearing stress is between zero and $0.792 \mathrm{MPa}$.

Figures 6.24 and 6.25 show the von Mises stresses in the steel at Increments 8 and 96, respectively. As noted above, when modeling the core of the bearing, we were not able to get convergence beyond $14.3 \%$ compressive engineering strain, most likely due to the severe distortion of the rubber elements adjacent to the core. Therefore, we neglected the core and prevented movement in the R-direction of all elements, rubber and steel, that are adjacent to the core. Therefore, the stresses and strains near the core 
will overstate the actual stresses and strains in the bearing. In the actual bearing, the shims are not constrained against movement in the $\mathrm{R}$-direction, and the core rubber separates from the shims, relieving stresses on the shims along the core. Shim failure in the actual bearing does not occur at the core, but actually occurs less than halfway from the core to the cover layer. Figure 6.26 shows the equivalent plastic strain at Increment 96. The highest plastic strains are at the interior of the shim near the midheight of the bearing, as we would expect. In spite of these inaccurate boundary conditions, as we move farther from the core the stresses and strains should more closely approximate the actual values. This is clear when we look at the deformed shape of the bearing. The simulated shim deformation accurately predicts the actual shim deformation as we move toward the cover layer of the bearing.

\subsection{Discussion of Differences in Initial Stifiness of Finite Element Model versus Experiment}

Figure 6.27 shows the stress-strain curve in the region prior to the abrupt change in the stiffness. Why is the finite element model significantly stiffer than experiment? In the experimental results, low stiffness at low stresses is present in each of the bearings. This initial low stiffness is due in part or entirely to plate and head misalignment, and/or the endplates being out of parallel. This effect has not been modeled. Other possible causes of the initial low stiffness may include prestresses of the rubber and steel due to the vulcanization process, and prestresses of the steel due to cold rolling.

\subsection{Results of Finite Element Analysis on Scale Models and Geometric Dissimilarities}

The actual geometry of the $1 / 2$ and $1 / 4-$ Scale models was reproduced in the finite element models, including the geometric dissimilarities discussed in Section 2.1.1. Figure 6.28 shows the $1 / 2$-Scale experimental bearing stress-strain curve, and the finite element models using the compressible rubber model (volumetric data CS7) with two different steel models, the first model based on the 16-gage sandblasted steel specimen SB1-16, and the second model based on the 12-gage chemlocked steel specimen CL1-12. The responses of the finite element models are virtually the same, reflecting the similarity of the steel stress-strain curves, as shown in Figure 6.29. The yield stresses for the steel models are virtually identical (261.9 MPa for steel specimen CL1-12 versus $260.5 \mathrm{MPa}$ for specimen SB1-16). This is reflected in the nearly identical shape of the bearing stress-strain curve up to initial yielding of the shims, as shown in Figure 6.28. The actual bearing failed at a compressive strain of $0.227 \mathrm{~m} / \mathrm{m}$ and a compressive stress of $88.7 \mathrm{MPa}$. The model based on steel specimen SB1-16 (1/2Scale_RM1_CS7_SB116) has a compressive stress of $90.3 \mathrm{MPa}$ at the failure strain of 0.227 , and for the model based on steel specimen CL1-12 the compressive stress at the failure strain is $90.8 \mathrm{MPa}\left(1 / 2-S c a l e \_R M 1 \_C S 7 \_C L 112\right)$ The secant modulus at the point of initial shim yielding is $730 \mathrm{MPa}$ for the actual test versus $1033 \mathrm{MPa}$ for both finite element models. The models overestimated the bearing stiffness in this region by $41.5 \%$. The slope of a straight line drawn from initial shim yielding to bearing failure for the actual test is $263 \mathrm{MPa}$ versus $252 \mathrm{MPa}$ for 1/2-Scale_RM1_CS7_CL112 and $249 \mathrm{MPa}$ for 1/2-Scale_RM1_CS7_SB116.

Figure 6.30 shows the 1/4-Scale experimental bearing stress-strain curve, and the finite element models using the compressible rubber model (volumetric data CS7) with two different steel models, the first model based on the 22-gage sandblasted steel 
specimen SB2-22, and the second model based on the 12-gage chemlocked steel specimen CL1-12. The response of the finite element models reflects the differences of the steel stress-strain curves, as shown in Figure 6.31. The yield stress for steel specimen SB2-22 is $289.9 \mathrm{MPa}$, or $10.7 \%$ larger than the yield stress of $261.9 \mathrm{MPa}$ for specimen CL112. The shims yielded in the bearing test at approximately $51.5 \mathrm{MPa}$, but for 1/4-Scale_RM1_CS7_CL112 initial yielding occured at $42.9 \mathrm{MPa}$ and for $1 / 4$ Scale_RM1_CS7_SB222 at $55.3 \mathrm{MPa}$. The actual bearing failed at a compressive strain of $0.281 \mathrm{~m} / \mathrm{m}$ and a compressive stress of $96.7 \mathrm{MPa}$. Model

1/4 Scale_RM1_CS7_SB222 has a compressive stress of $105 \mathrm{MPa}$ at the failure strain of 0.281, and in model 1/4-Scale_RM1_CS7_CL112 the compressive stress at the failure strain is $100.4 \mathrm{MPa}$. The secant modulus at the point of initial shim yielding is $472 \mathrm{MPa}$ for the actual test versus approximately $975 \mathrm{MPa}$ for both finite element models. The finite element models overestimated the bearing stiffness in this region by $207 \%$. However, as noted in Section 2.6.6, the tangent stiffness of the experimental curve is $930 \mathrm{MPa}$ just prior to the initial yielding of the shims, which is very close to the slope of each finite element run. This is apparent in Figure 6.30. The initial low stiffness at low stresses is present in each of the experimental bearings tested, but is particularly severe for the 1/4-Scale Bearing. As discussed in Section 6.3 , this initial low stiffness may be due to plate and head misalignment, and/or the end plates being out of parallel. These affects would be magnified for smaller scale bearings, since they are made to the same tolerances as full-scale bearings. The slope of a straight line drawn from initial shim yielding to bearing failure for the actual test is $263 \mathrm{MPa}$ versus $223 \mathrm{MPa}$ for 1/4 Scale_RM1_CS7_SB222 and $242 \mathrm{MPa}$ for 1/4 Scale_RM1_CS7_CL112. The finite element results are accurate, as is apparent by inspection of Figure 6.30 , especially after taking into account the initial low stiffness of the actual 1/4-Scale Bearing.

As noted in Section 2.1.1, the B-Bearings are dissimilar in three ways: shim thickness, center alignment hole diameter, and cover layer thickness. The $1 / 4$ and $1 / 2$ Scale shims are 22-gage and 16-gage, respectively, or nominally $0.762 \mathrm{~mm}(0.03 \mathrm{in})$ and $1.524 \mathrm{~mm}(0.06 \mathrm{in})$ thick, and are therefore similar in thickness, but the Prototype shim is 12 -gage, or nominally $2.667 \mathrm{~mm}$ thick $(0.105 \mathrm{in})$, so it is $0.381 \mathrm{~mm}(0.015 \mathrm{in})$ thinner, or $12.5 \%$ thinner, than it should be in order to have a geometrically similar thickness. The diameter of the center alignment hole for the Prototype is $50.1 \mathrm{~mm}$, but the diameters of the center alignment holes for the 1/2-Scale and 1/4-Scale models are each $30.1 \mathrm{~mm}$. The $1 / 2$-Scale model's center alignment hole diameter is $20.1 \%$ larger than it should have been $(25.05 \mathrm{~mm})$ for geometric similarity, and the 1/4-Scale model's center alignment hole diameter is $141 \%$ larger than it should have been $(12.5025 \mathrm{~mm})$ for geometric similarity. The cover layer for the Prototype is $19 \mathrm{~mm}$ thick, and should be $9.5 \mathrm{~mm}$ thick for the $1 / 2$-Scale model, but is actually $12.5 \mathrm{~mm}$, or $32 \%$ too large, and should be $4.75 \mathrm{~mm}$ thick for the 1/4-Scale model, but is actually 19 $\mathrm{mm}$, or $300 \%$ too thick for strict geometric similarity to hold. For convenience, this information is compiled in Table 6.1 shown immediately below. 
Table 6.1 - Summary of geometric dissimilarities

\begin{tabular}{lccc}
\hline & Prototype (mm) & $1 / 2$ Scale (mm) & $1 / 4$ Scale (mm) \\
\hline Shim Thickness & & & \\
Actual Dimensions & 2.667 & 1.524 & 0.762 \\
For Geometric Similarity & 3.048 & 1.524 & 0.762 \\
\hline $\begin{array}{c}\text { Center Alignment Hole } \\
\text { Diameter }\end{array}$ & & \\
$\quad$ Actual Dimensions & 50.1 & 30.1 & 30.1 \\
For Geometric Similarity & 50.1 & 25.05 & 12.5025 \\
\hline Cover Layer Thickness & & & \\
Actual Dimensions & 19.0 & 12.5 & 19.0 \\
For Geometric Similarity & 19.0 & 9.50 & 4.75 \\
\hline
\end{tabular}

A finite element run was conducted for the Prototype using steel model CL1 12 , deviatoric rubber model RM1, and volumetric test data CS7, but with the shims. made geometrically similar to the $1 / 2$ and $1 / 4-S c a l e$ models; i.e. $3.048 \mathrm{~mm}$ thick (11 gage shims). Figure 6.32 shows the comparison of this run with each of the bearings as-is. Using geometrically similar shims for the prototype, the figure shows a large increase in the stress-strain response of the bearing between initial shim yielding and failure. For example, the stress at a strain of $0.25 \mathrm{~m} / \mathrm{m}$ was $10 \%$ higher using 11 gage shims. Furthermore, the response of the prototype, using similar shims, is nearly identical to the $1 / 4$ and $1 / 2$-Scale Bearing responses.

The geometric dissimilarities in the central alignment hole and cover layer are most pronounced with the 1/4-Scale Bearing, as shown in Table 6.1. As shown in Figure 6.32, in spite of these geometric dissimilarities, the response of the 1/4-Scale Bearing is nearly identical to the prototype, assuming geometrically similar shims. Figure 6.33 shows the separate effects of each of the dissimilarities, and they are seen, in this particular case, to cancel each other out. For example, making the core of the 1/4-Scale Bearing geometrically similar to the prototype (leaving all other geometry unchanged) increases the stress by approximately $3 \%$ at a strain of $0.25 \mathrm{~m} / \mathrm{m}$, and making the cover layer of the 1/4-Scale Bearing geometrically similar to the prototype (leaving all other geometry unchanged) decreases the stress by about the same amount at the same strain of $0.25 \mathrm{~m} / \mathrm{m}$. Clearly, the effects of the dissimilarities of the core and cover layer on the response of the 1/4-Scale Bearing are less important than the dissimilarity in the shim thickness. 


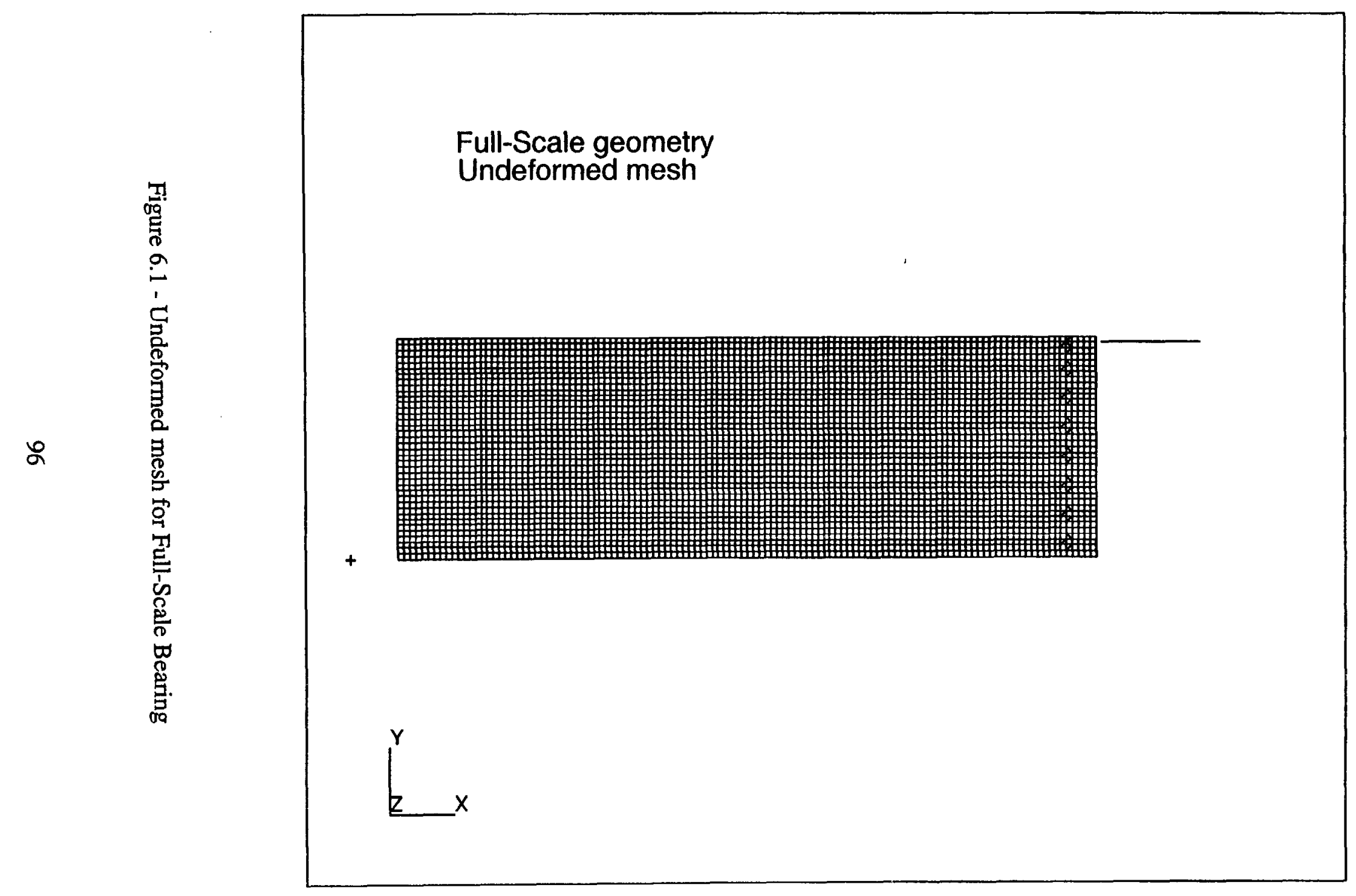




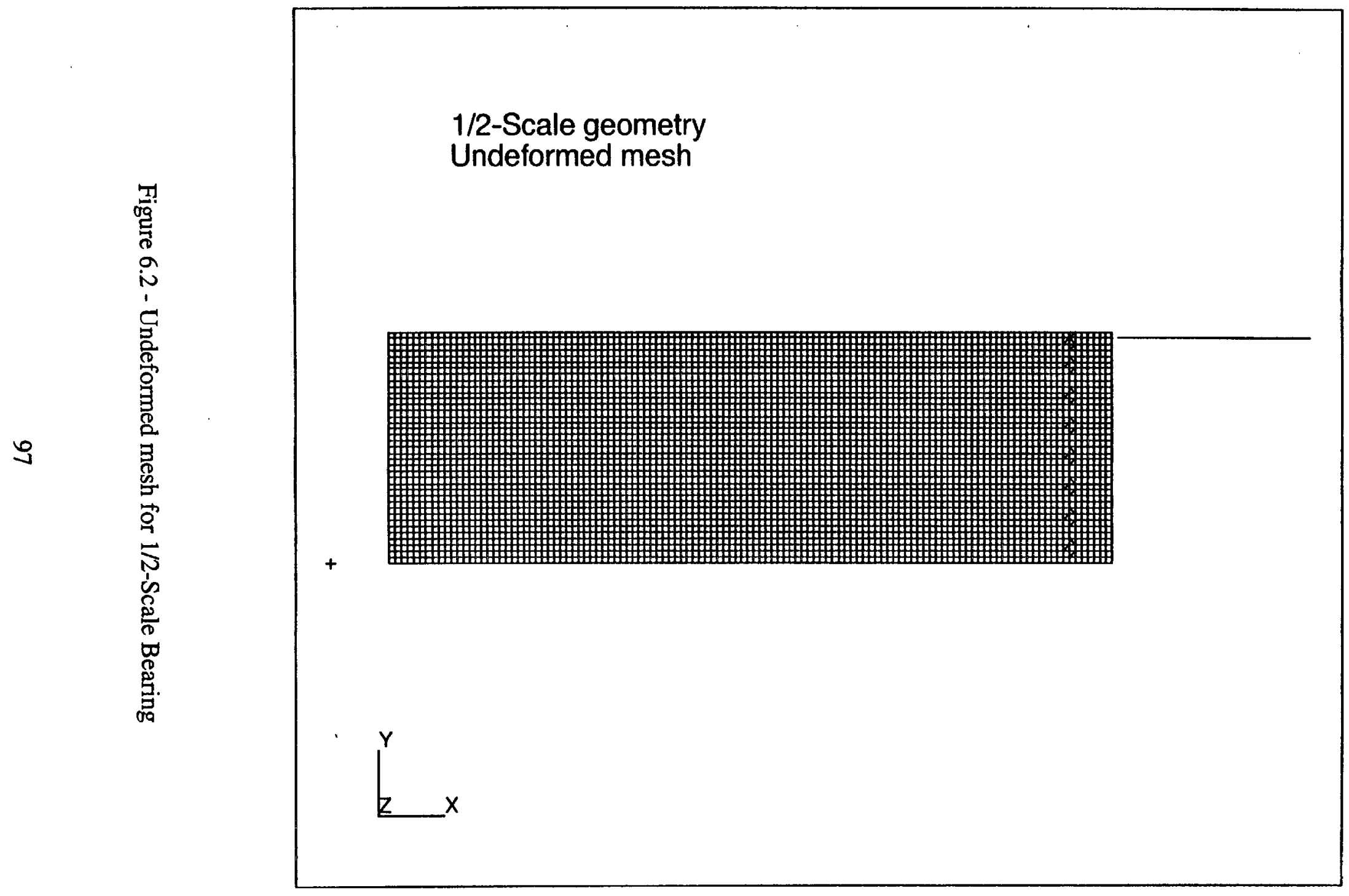


1/4-Scale geometry

$\infty$

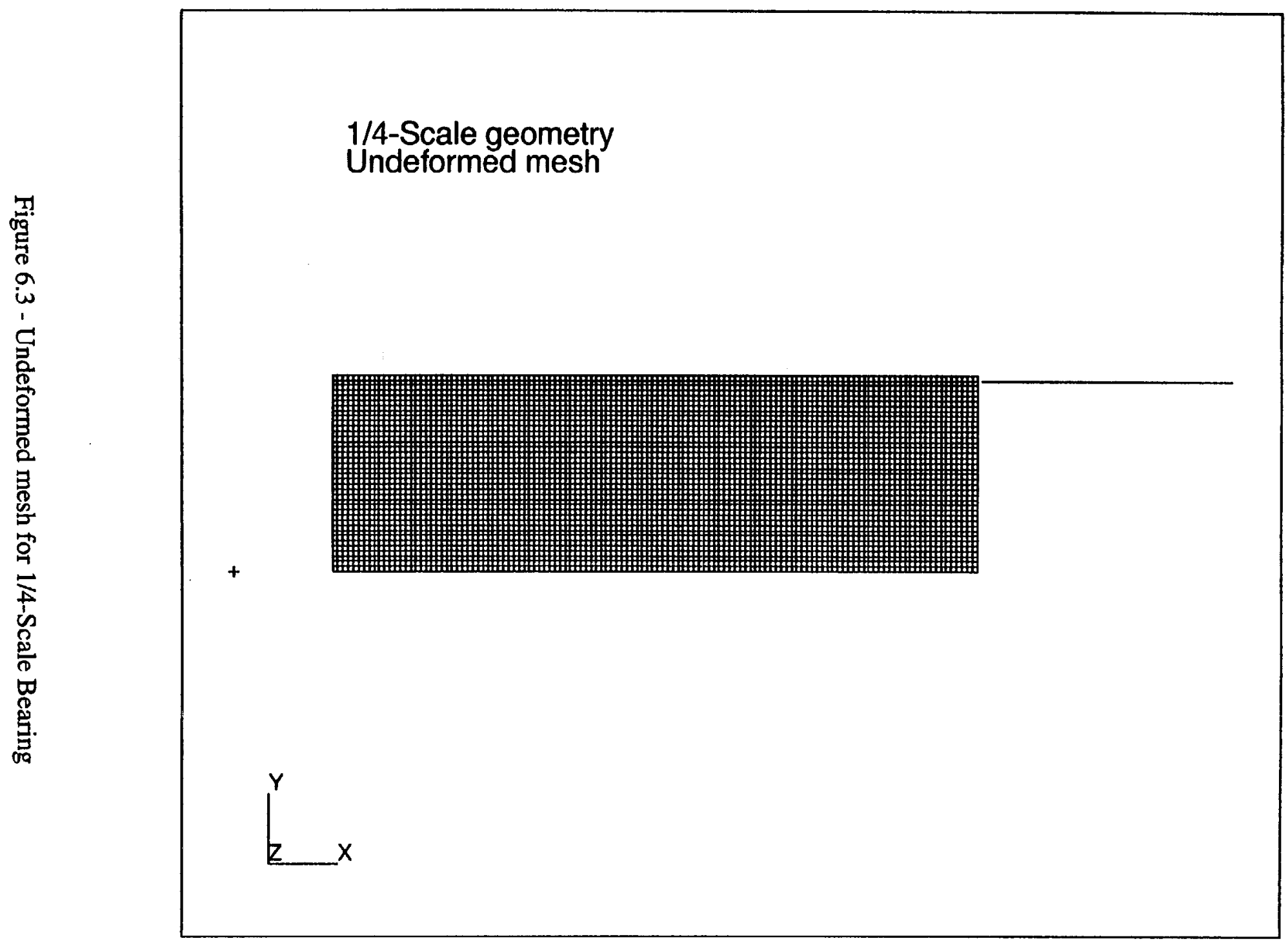




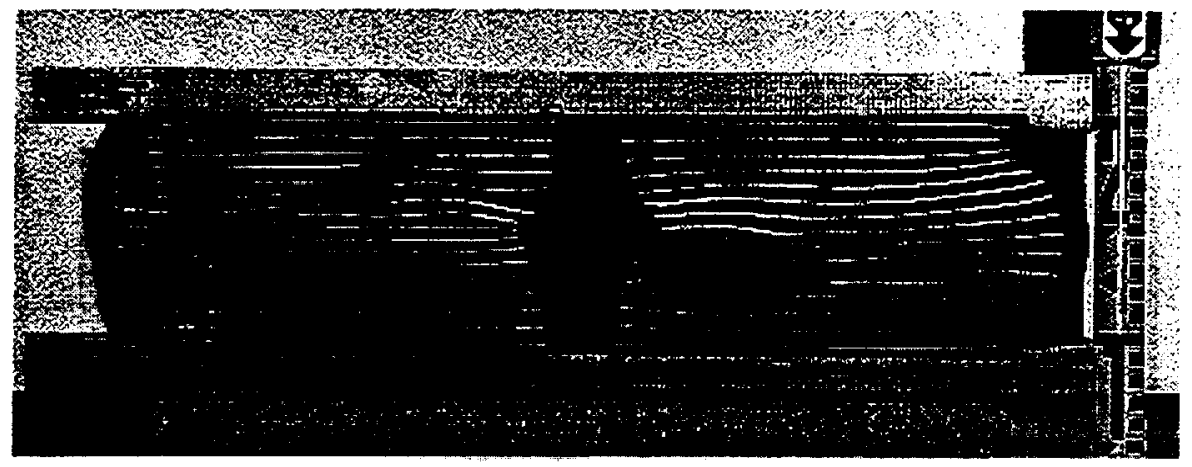

Figure 6.4 - Photograph of central slice of failed 1/2-Scale Bearing (B2) 


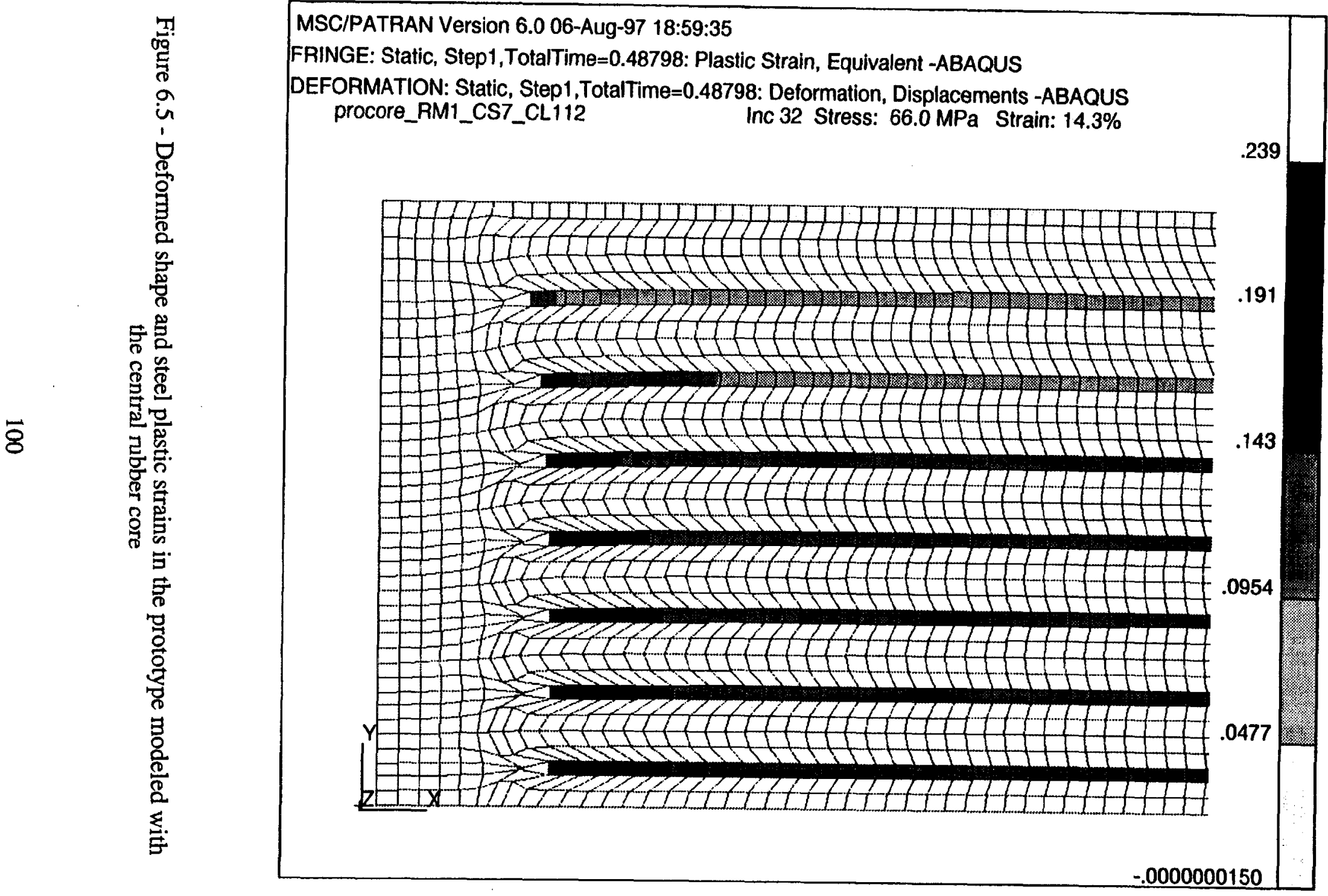




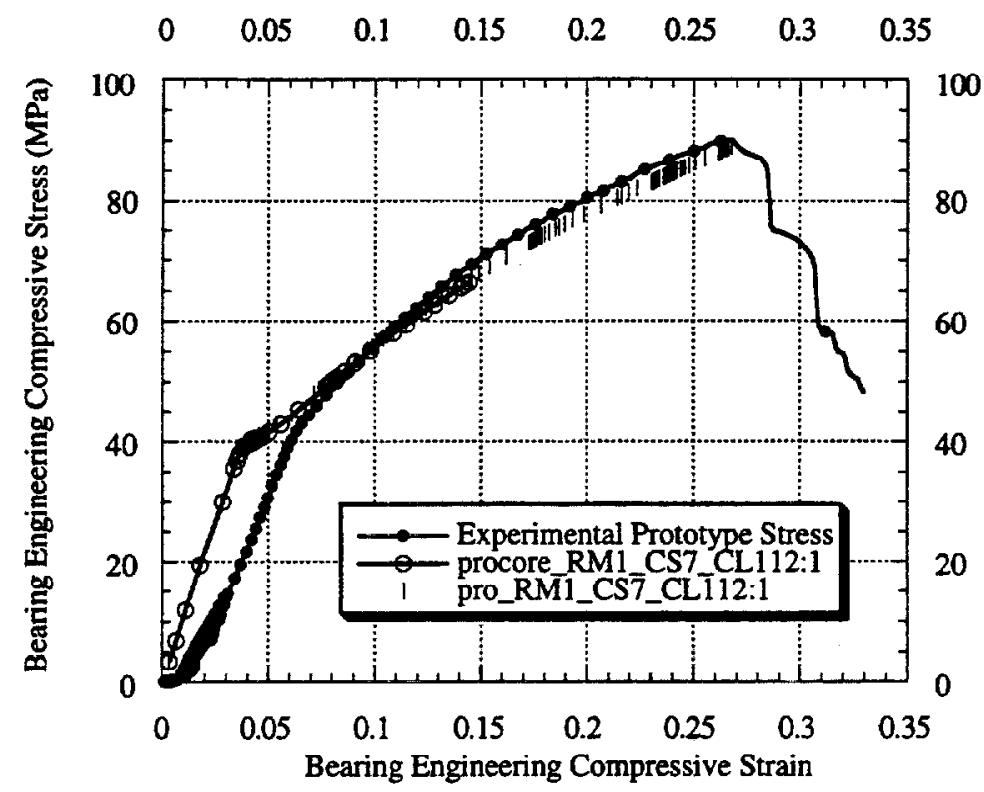

Figure 6.6 - Comparison for the prototype between the experimental engineering stress strain curve and ABAQUS finite element runs with and without the modeling of the core

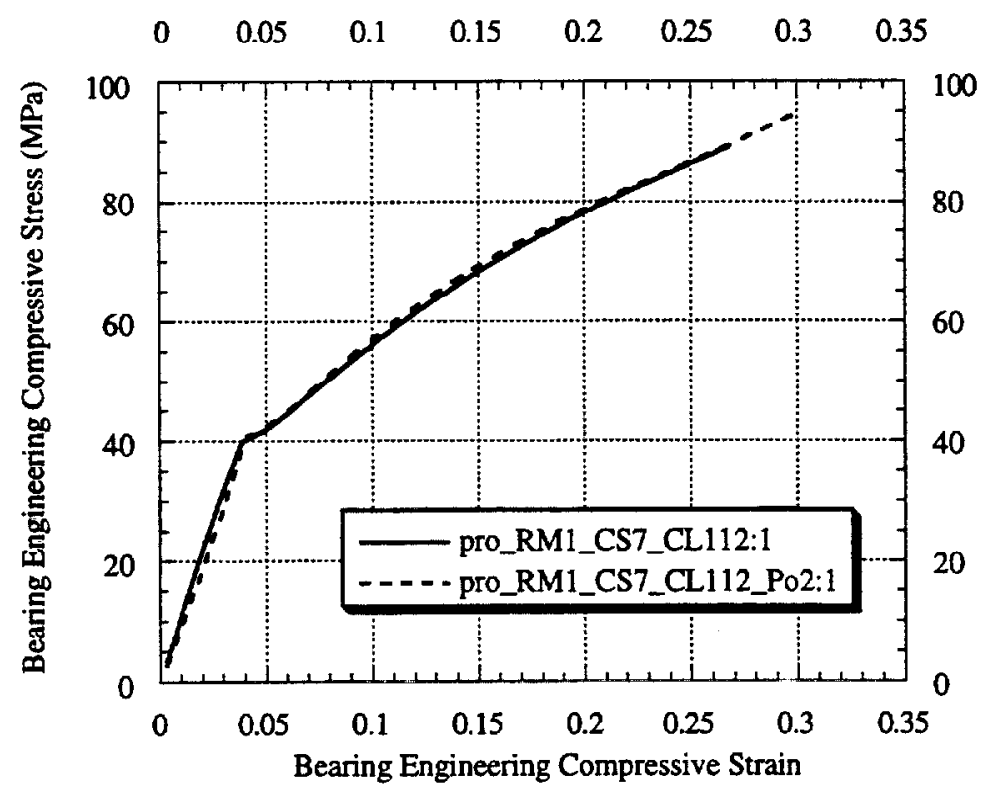

Figure 6.7 - Comparison of ABAQUS runs based on Ogden, $\mathrm{N}=6$ and Polynomial, $\mathrm{N}=2$ Strain Energy Functions 


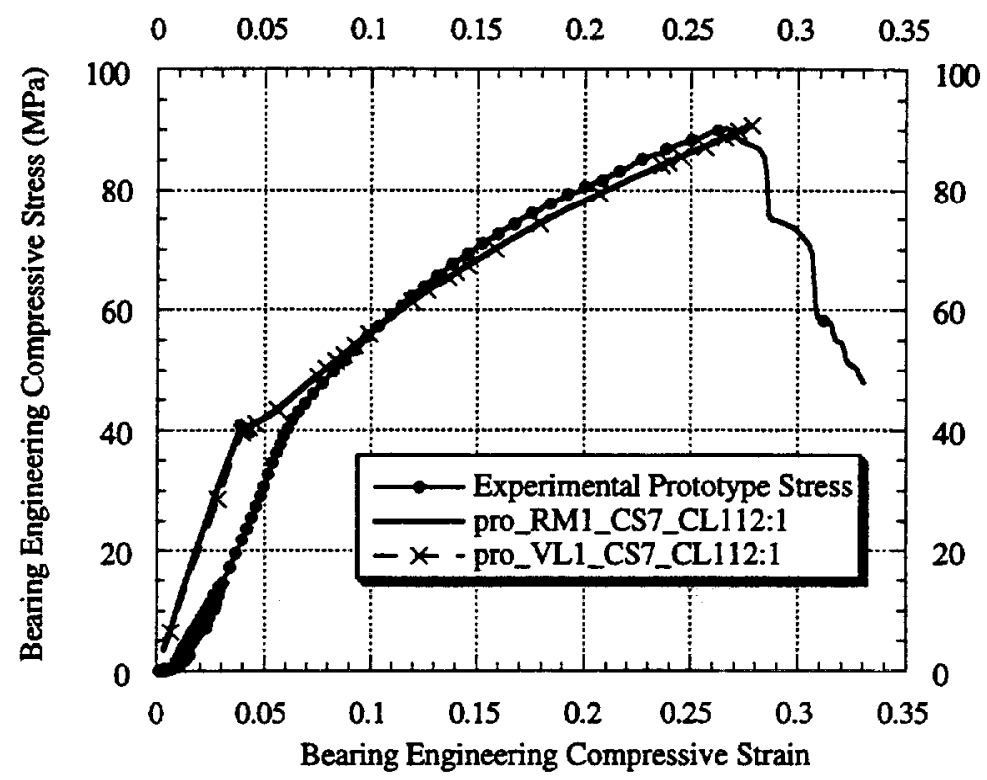

Figure 6.8 - Comparison for the prototype between the experimental engineering stress strain curve and the ABAQUS finite element runs based on RM1 and VL1

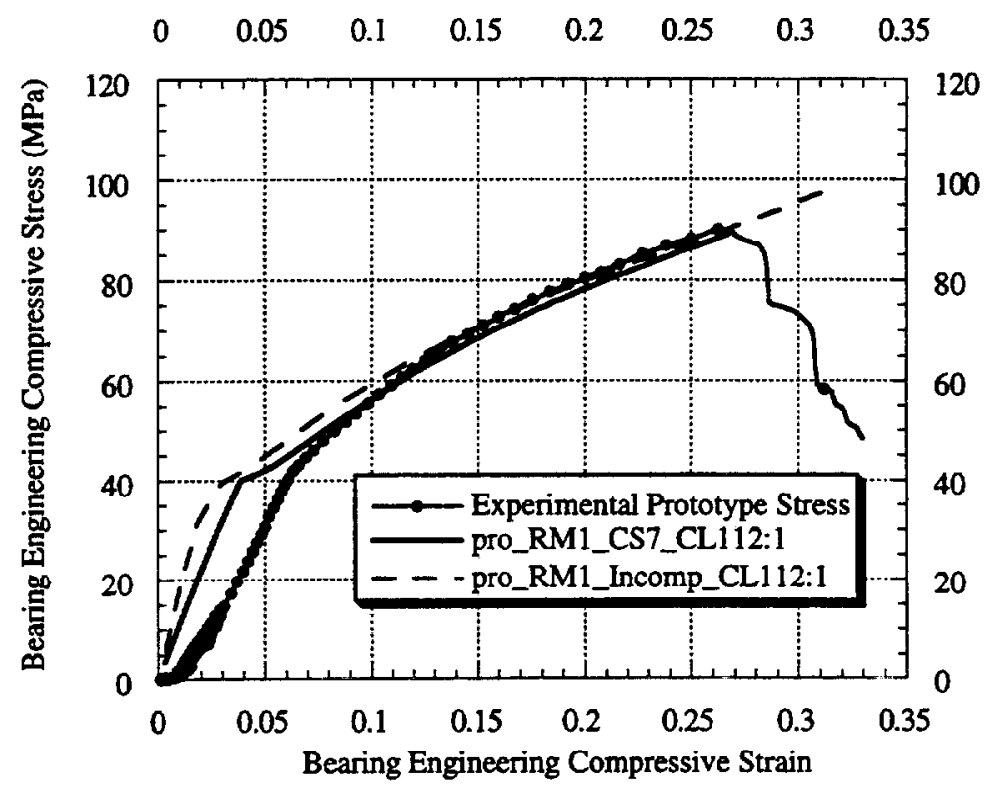

Figure 6.9 - Comparison for the prototype between the experimental engineering stress-strain curve and the ABAQUS finite element runs using a compressible versus an incompressible rubber model 


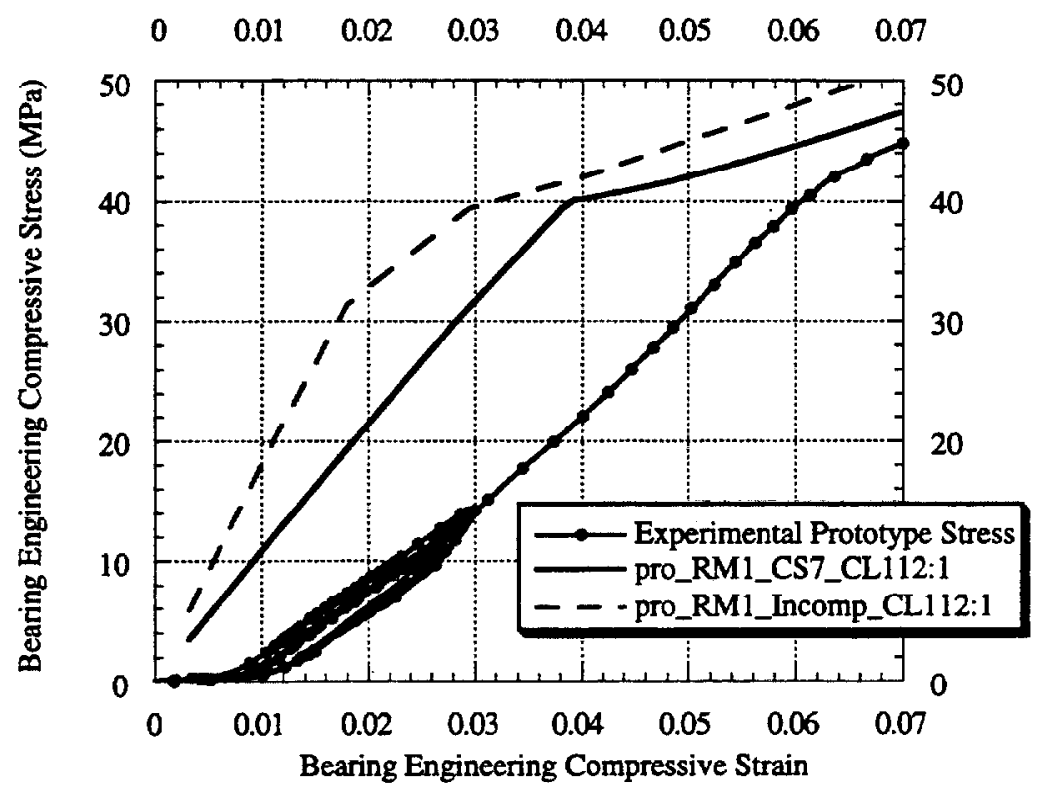

Figure 6.10 - Low strain region comparison for the prototype between the experimental engineering stress-strain curve and the ABAQUS finite element runs using a compressible versus an incompressible rubber model

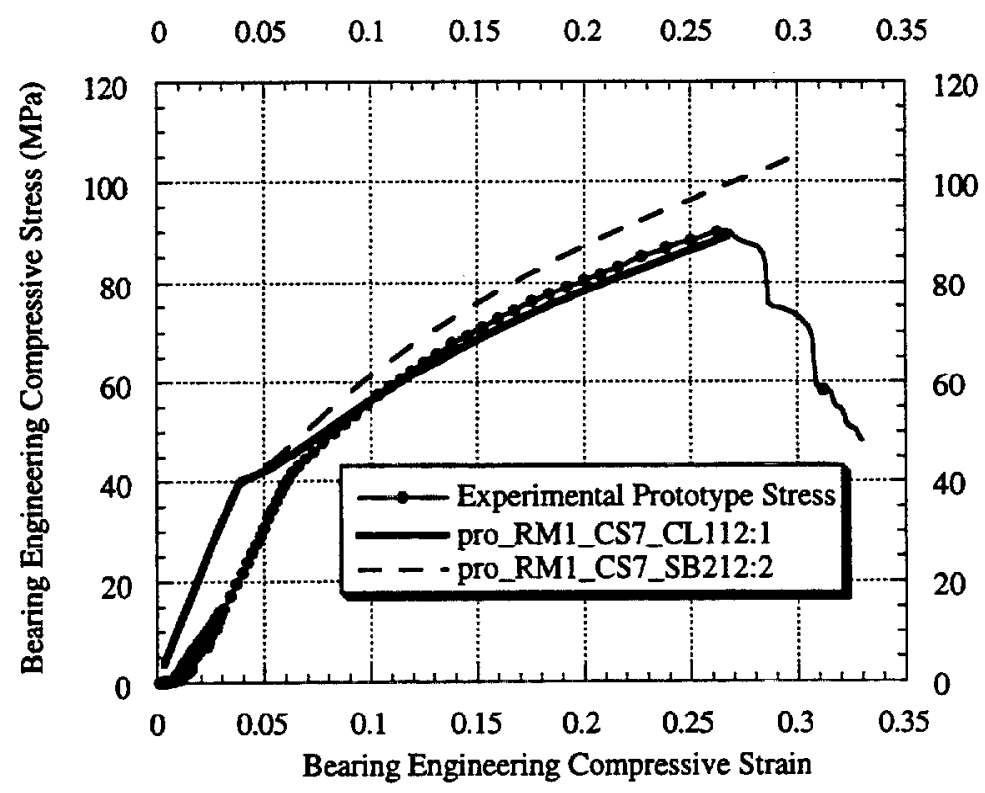

Figure 6.11 - Comparison for the prototype between the experimental engineering stress-strain curve and the ABAQUS finite element runs using the chemlocked steel data (CL1-12) versus the sandblasted steel data (SB212) 


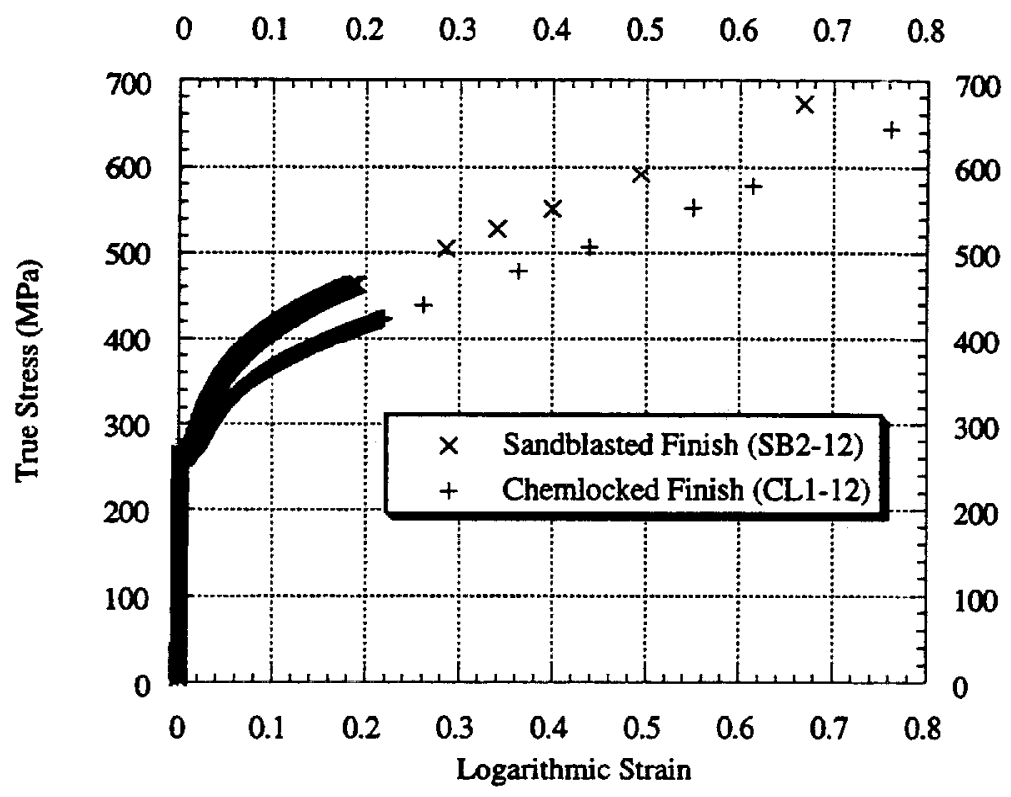

Figure 6.12 - Comparison between the true stress-strain curves of the chemlocked steel data (CL1-12) versus the sandblasted steel data (SB2-12) 


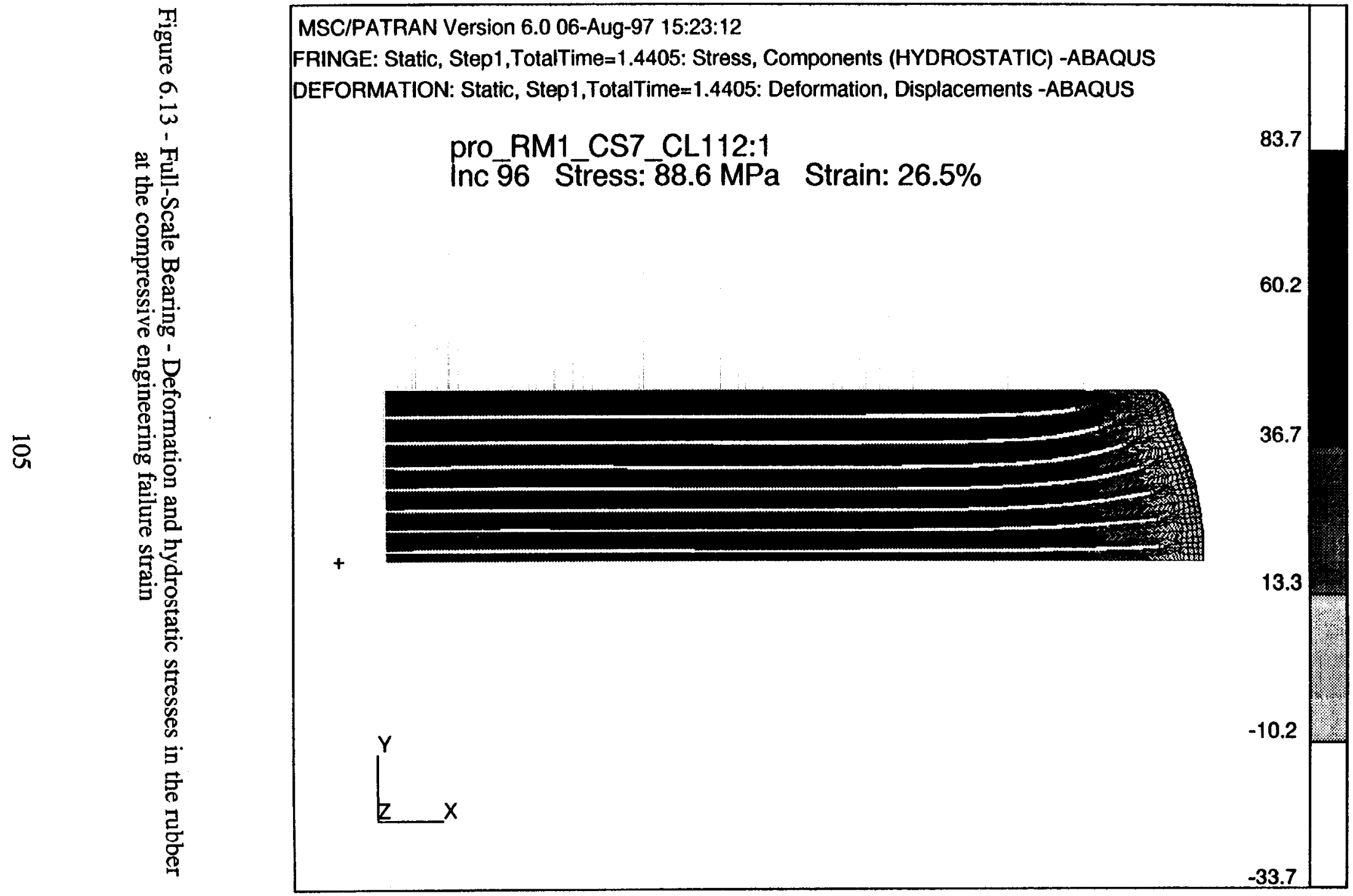




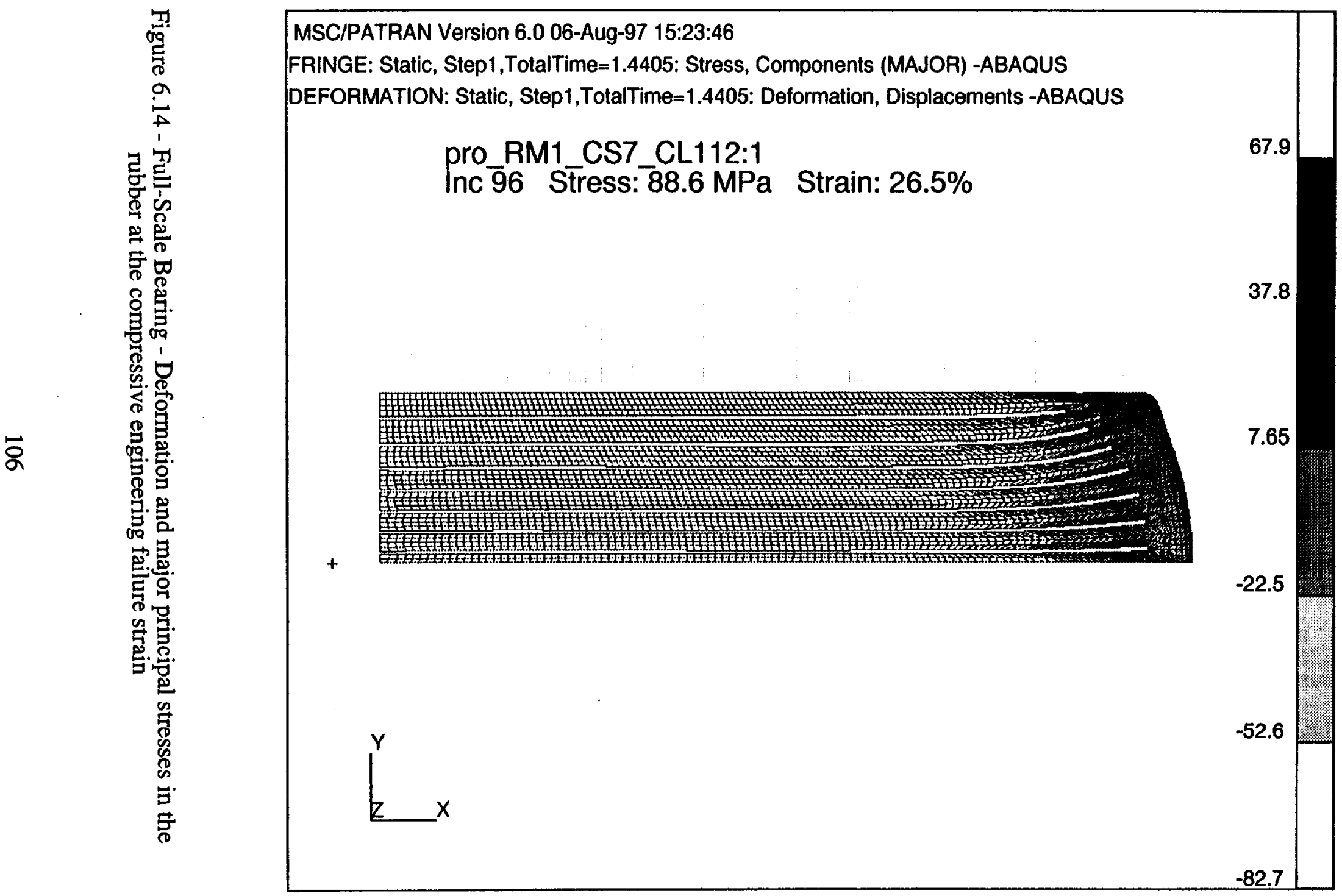




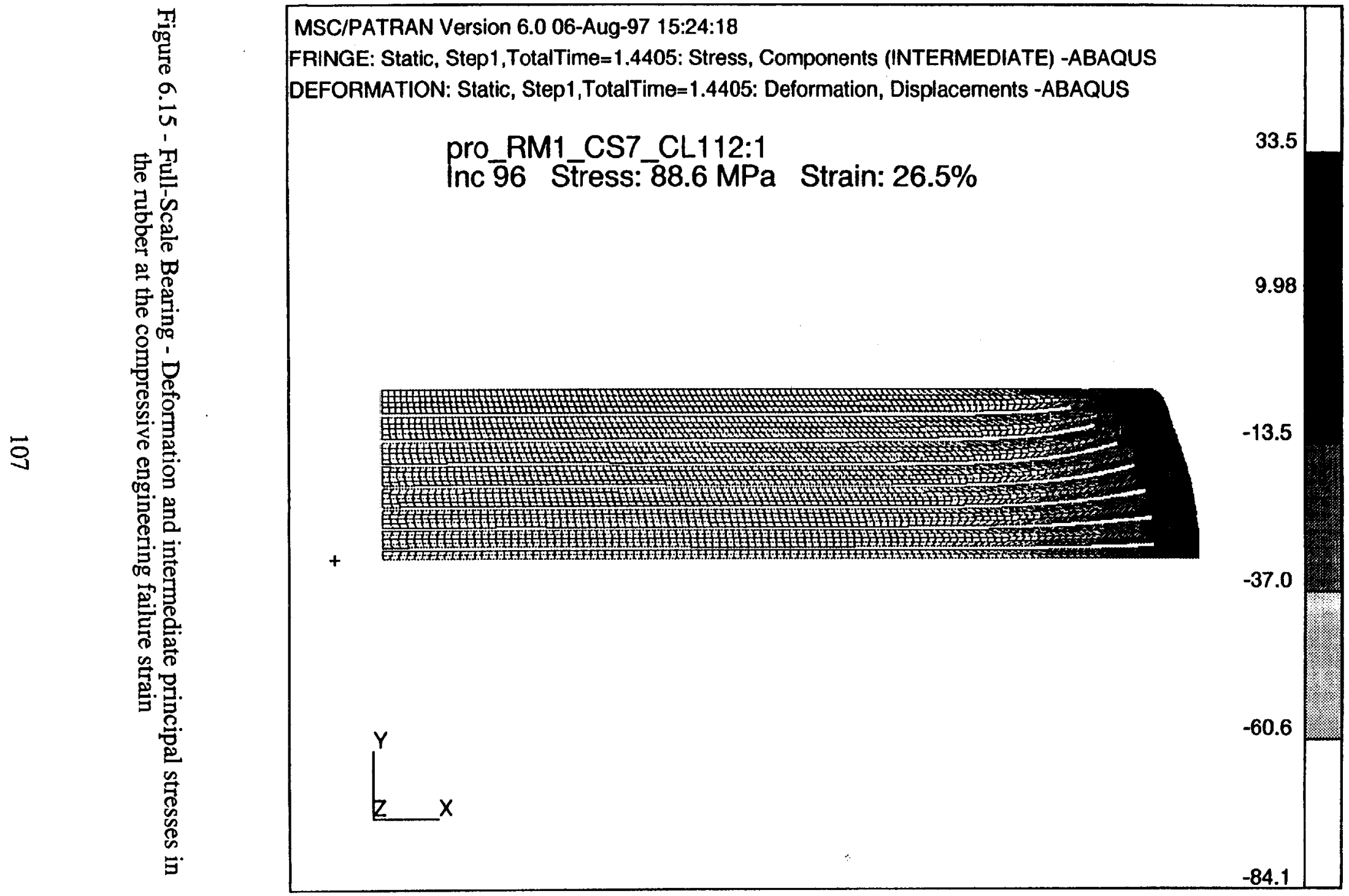




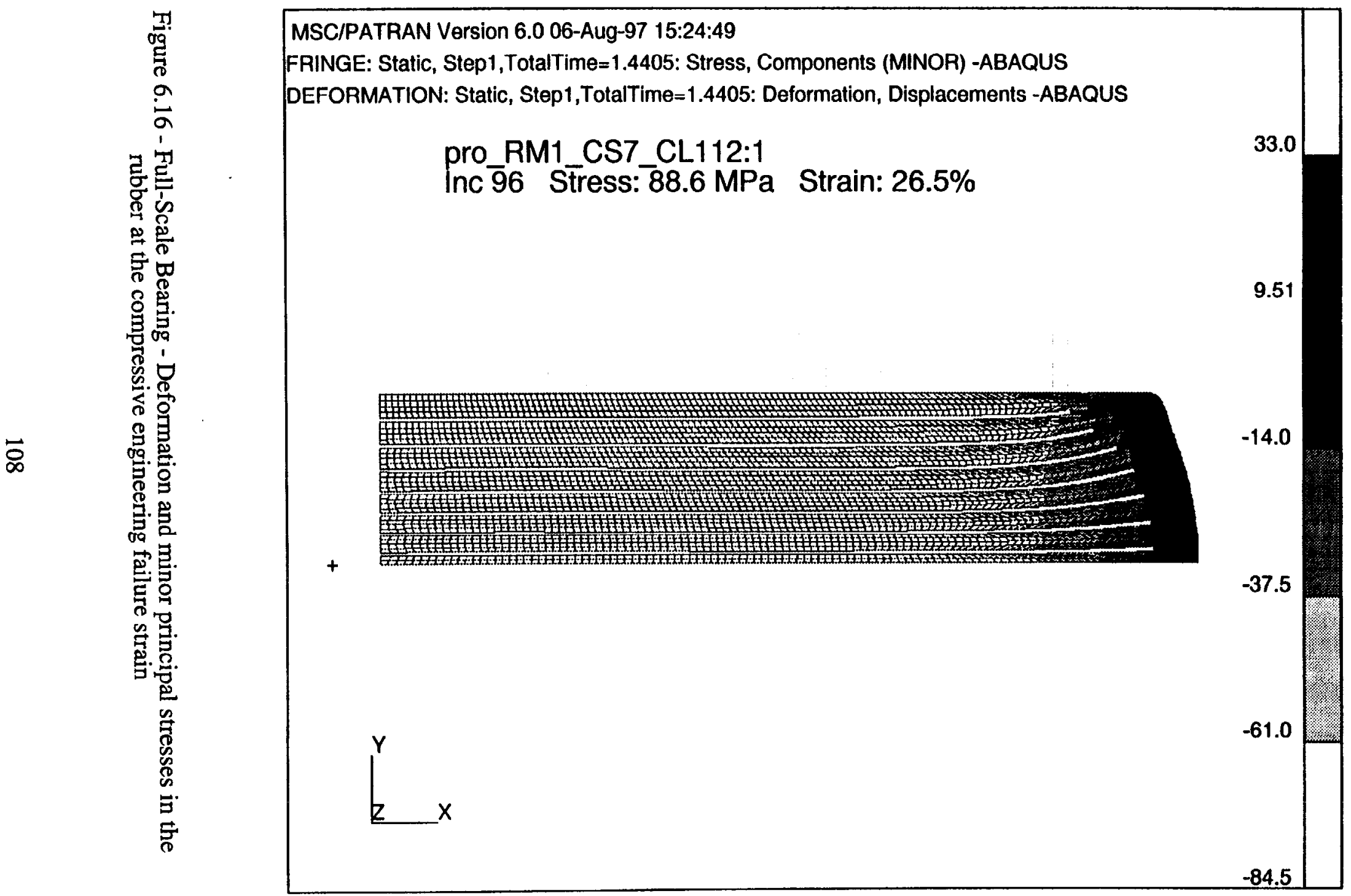




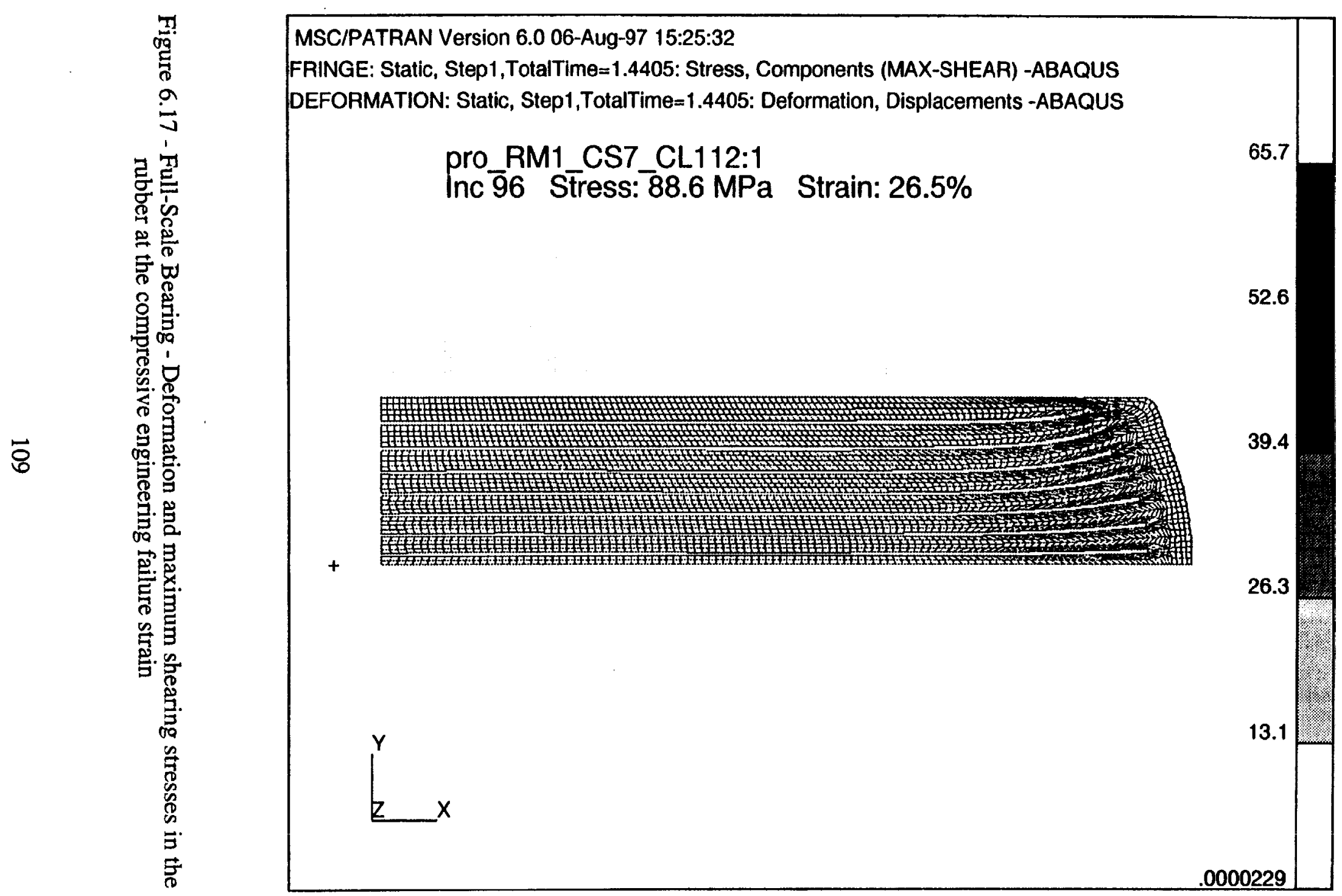




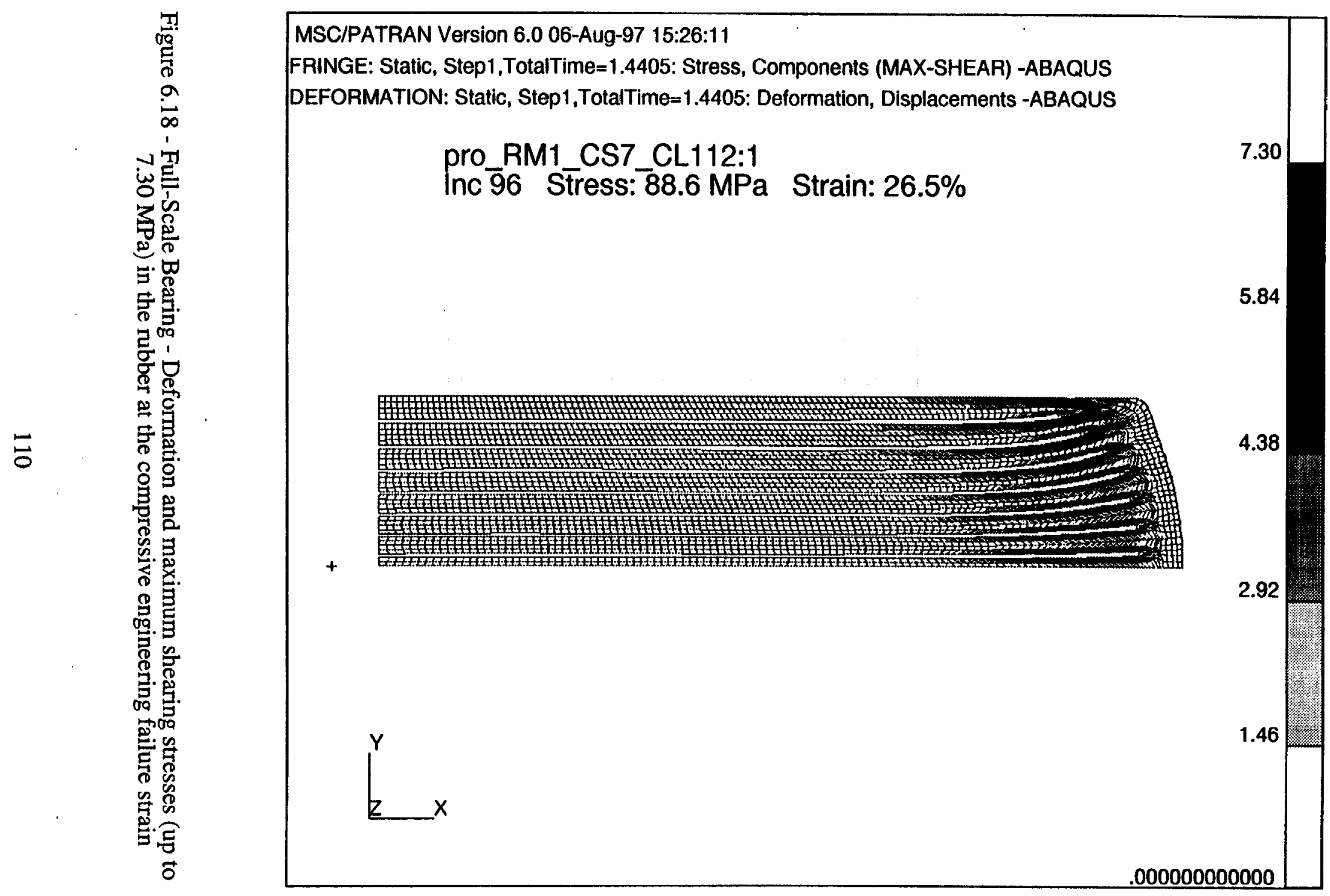




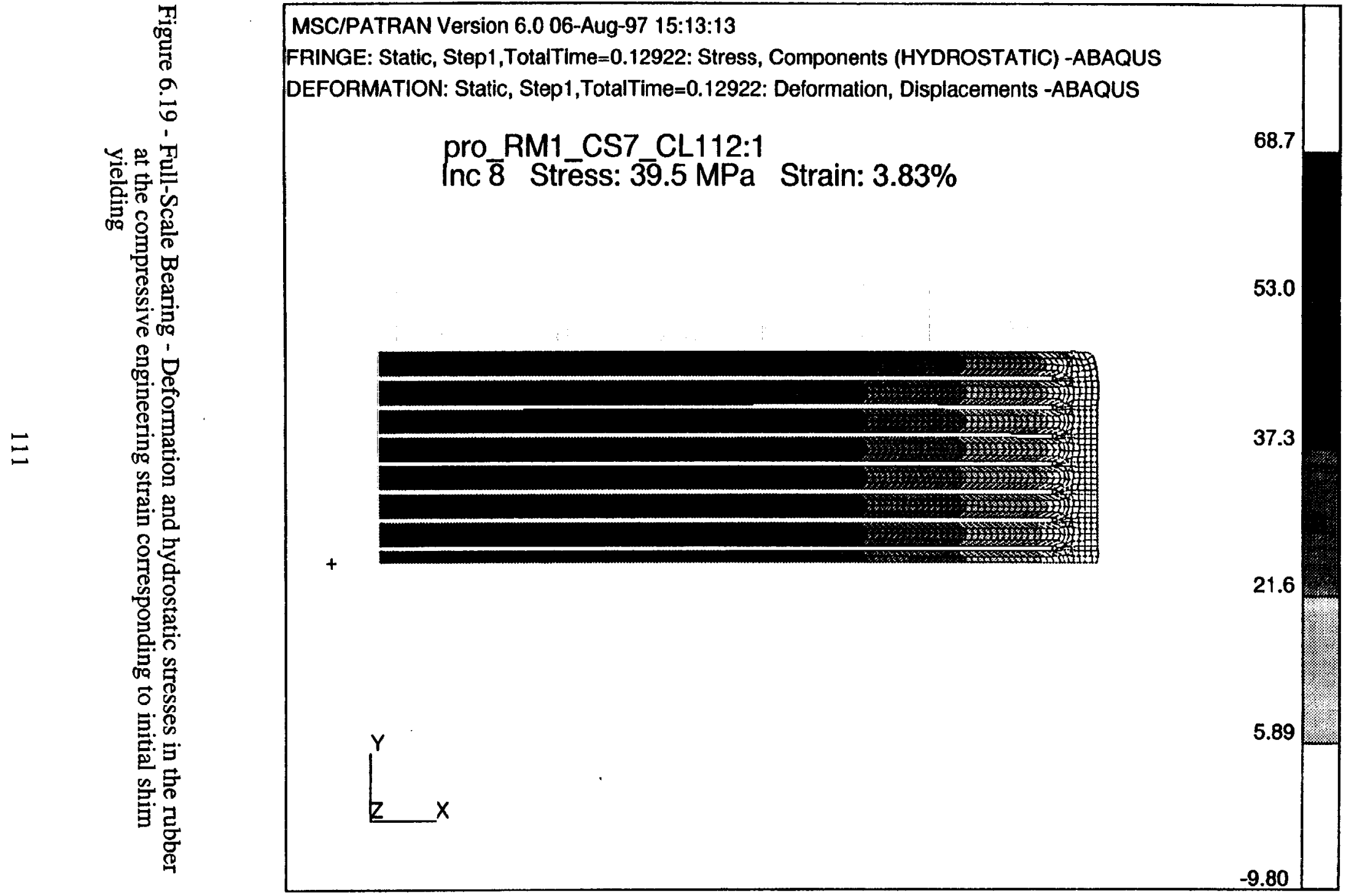




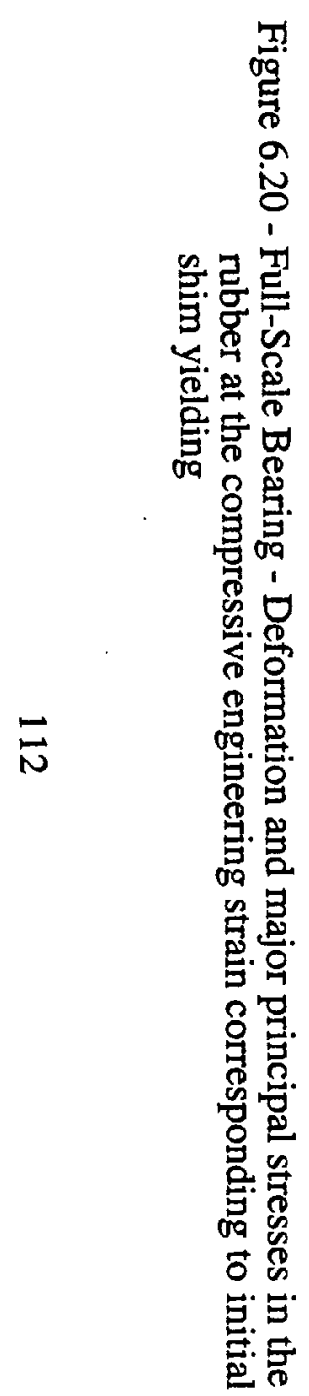

(1)

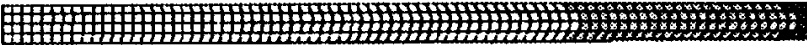

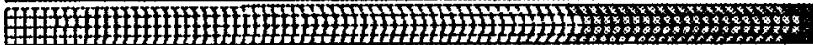

(G)

声

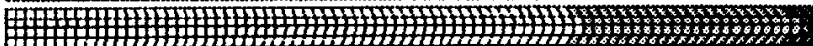

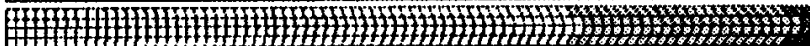

$+$

管

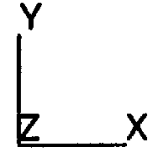




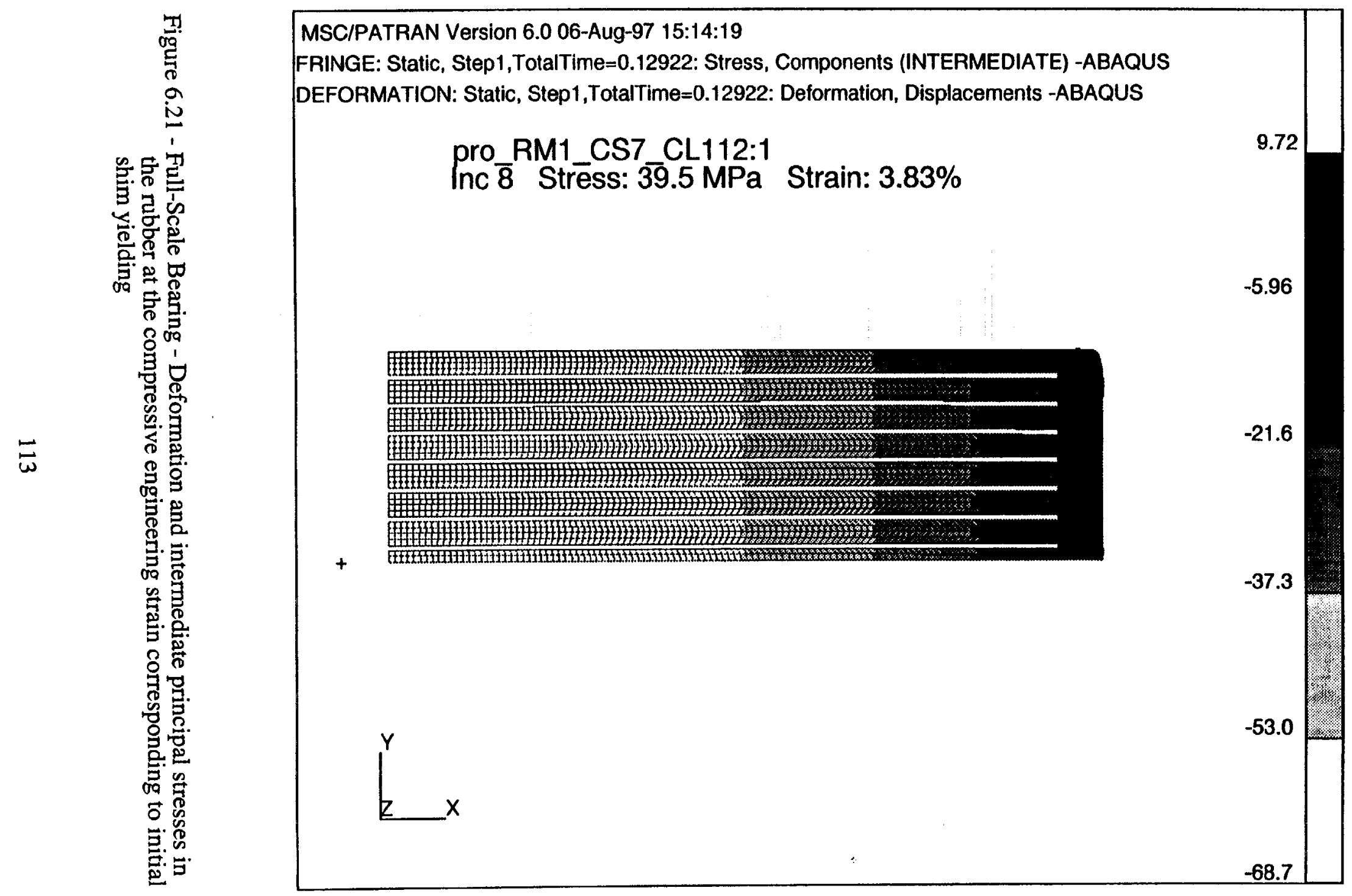




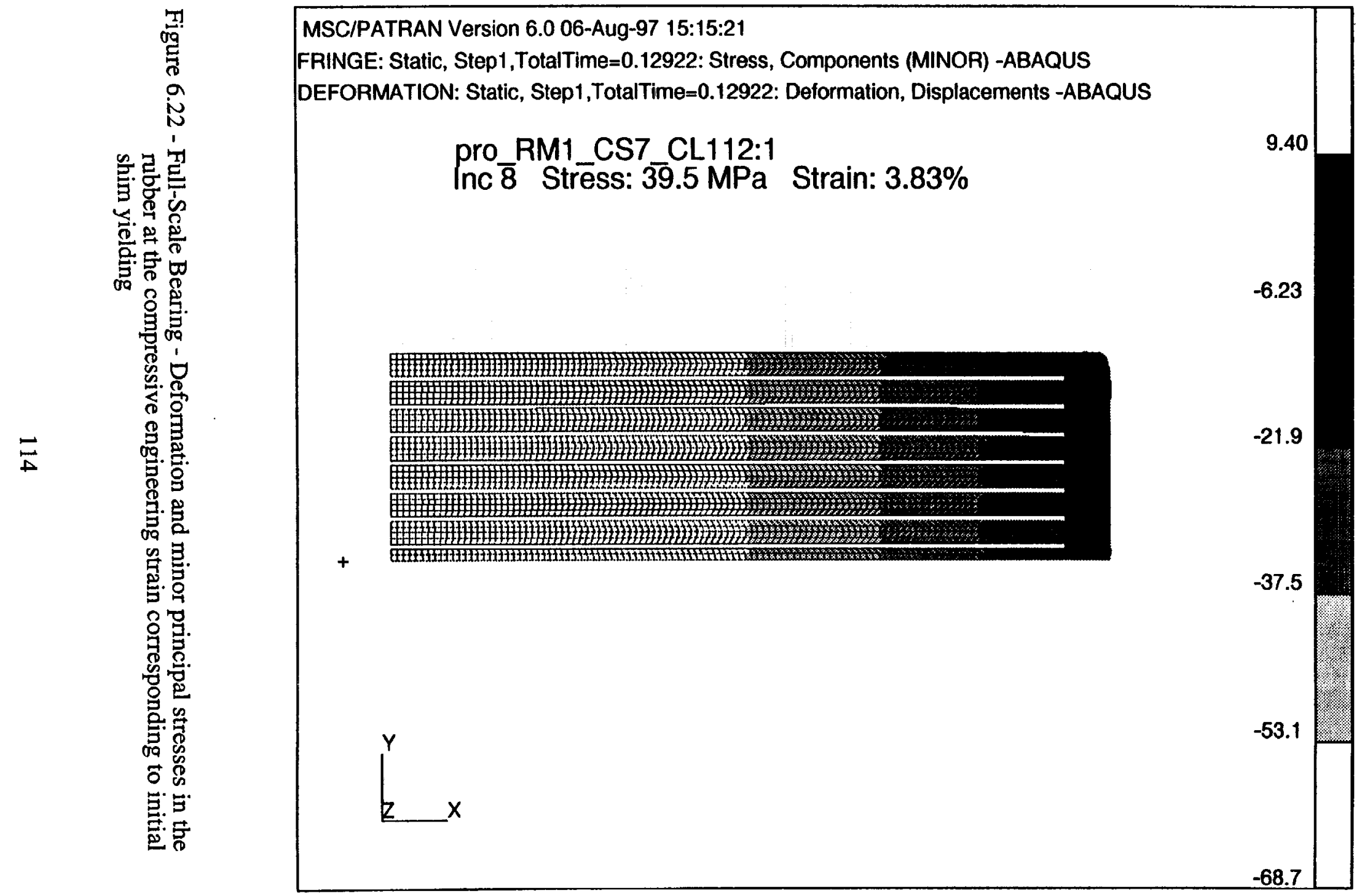




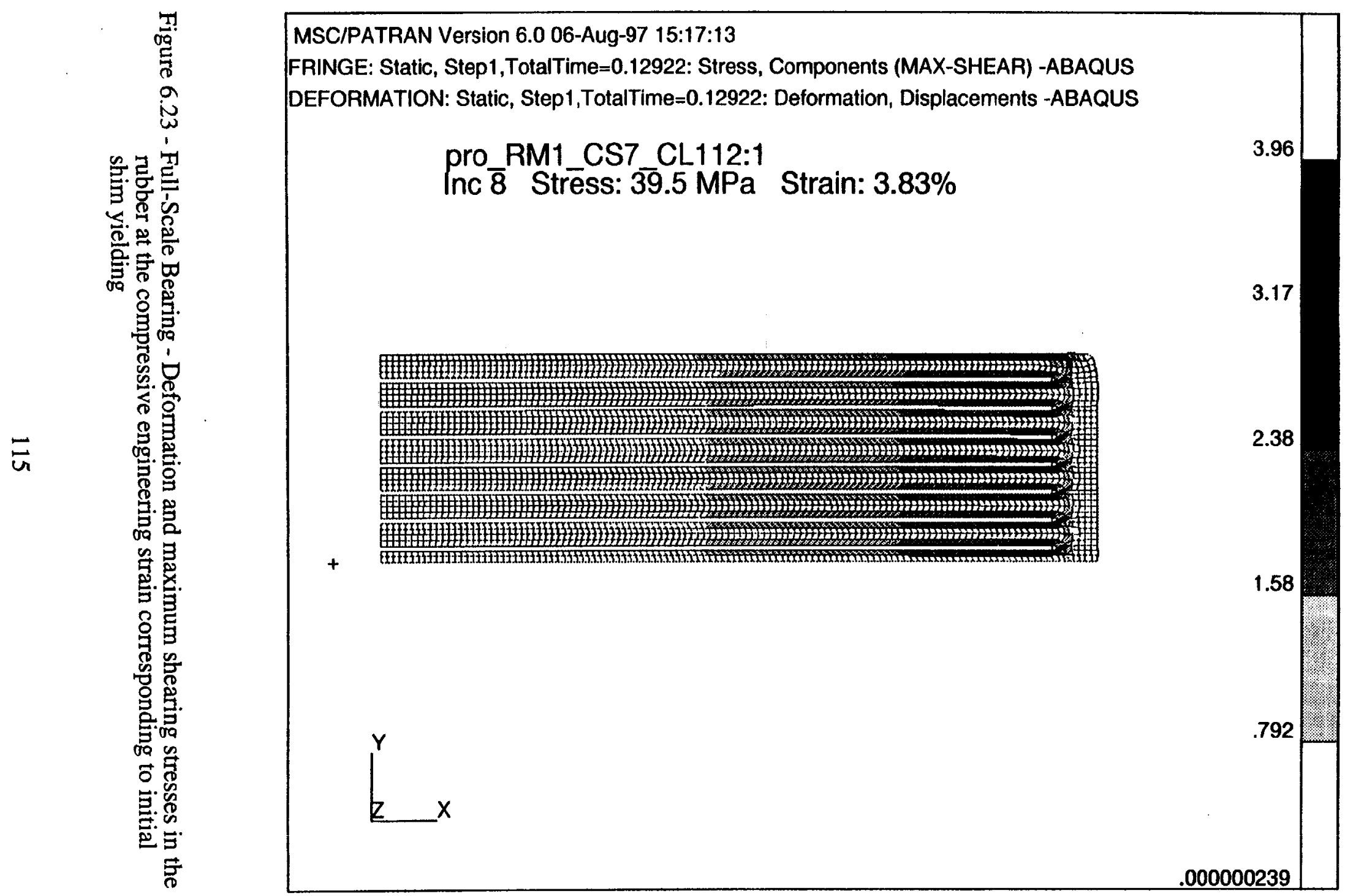




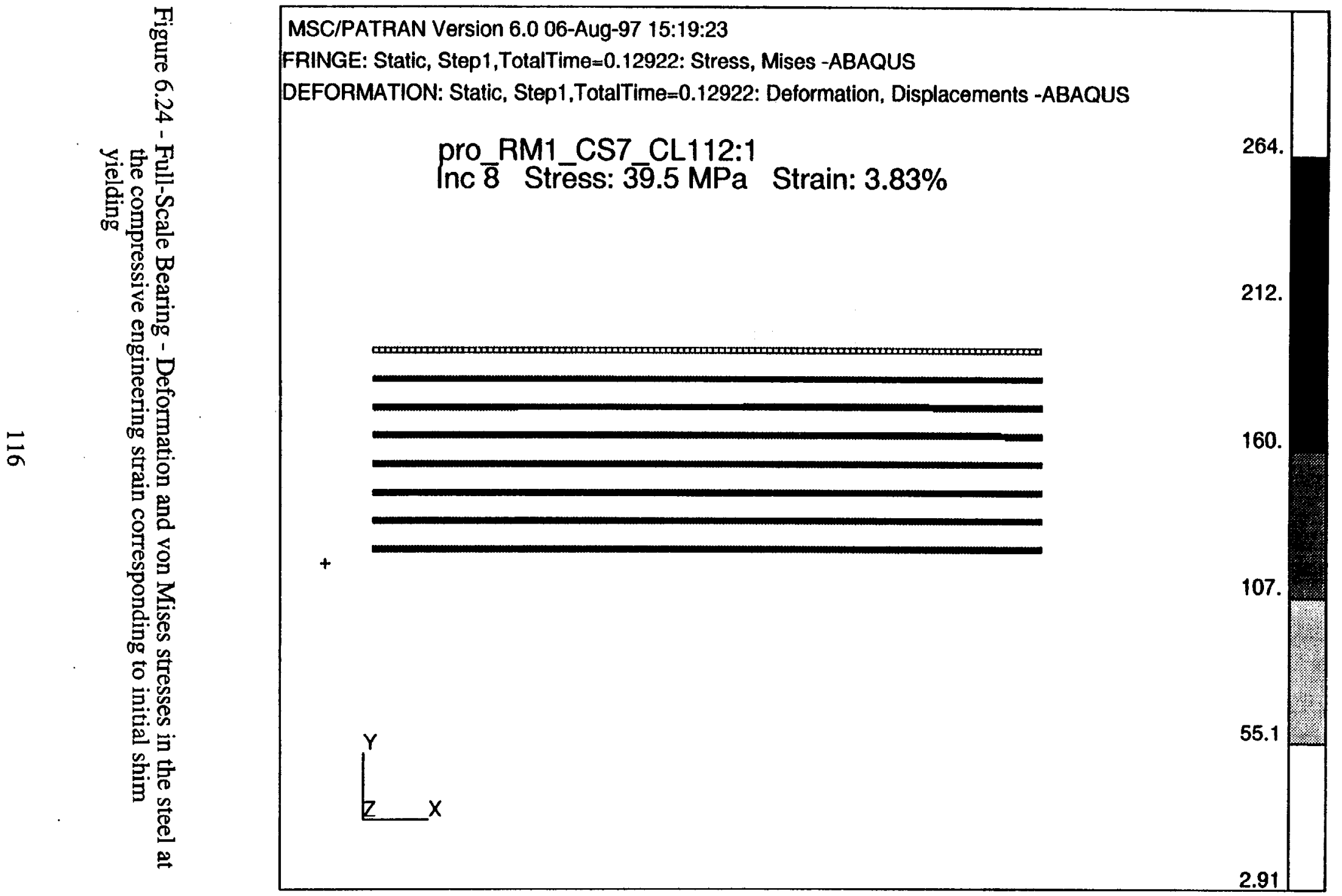




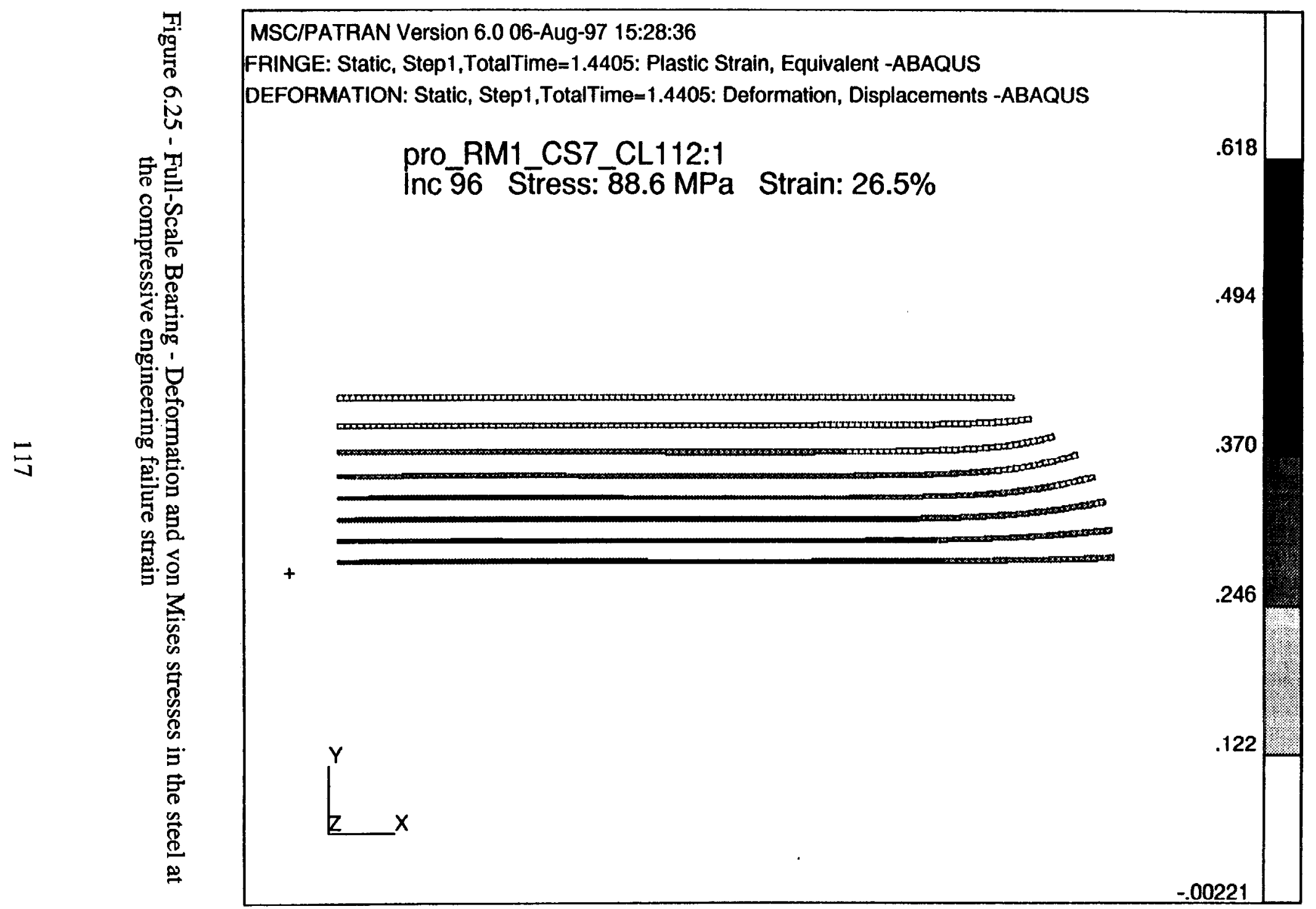




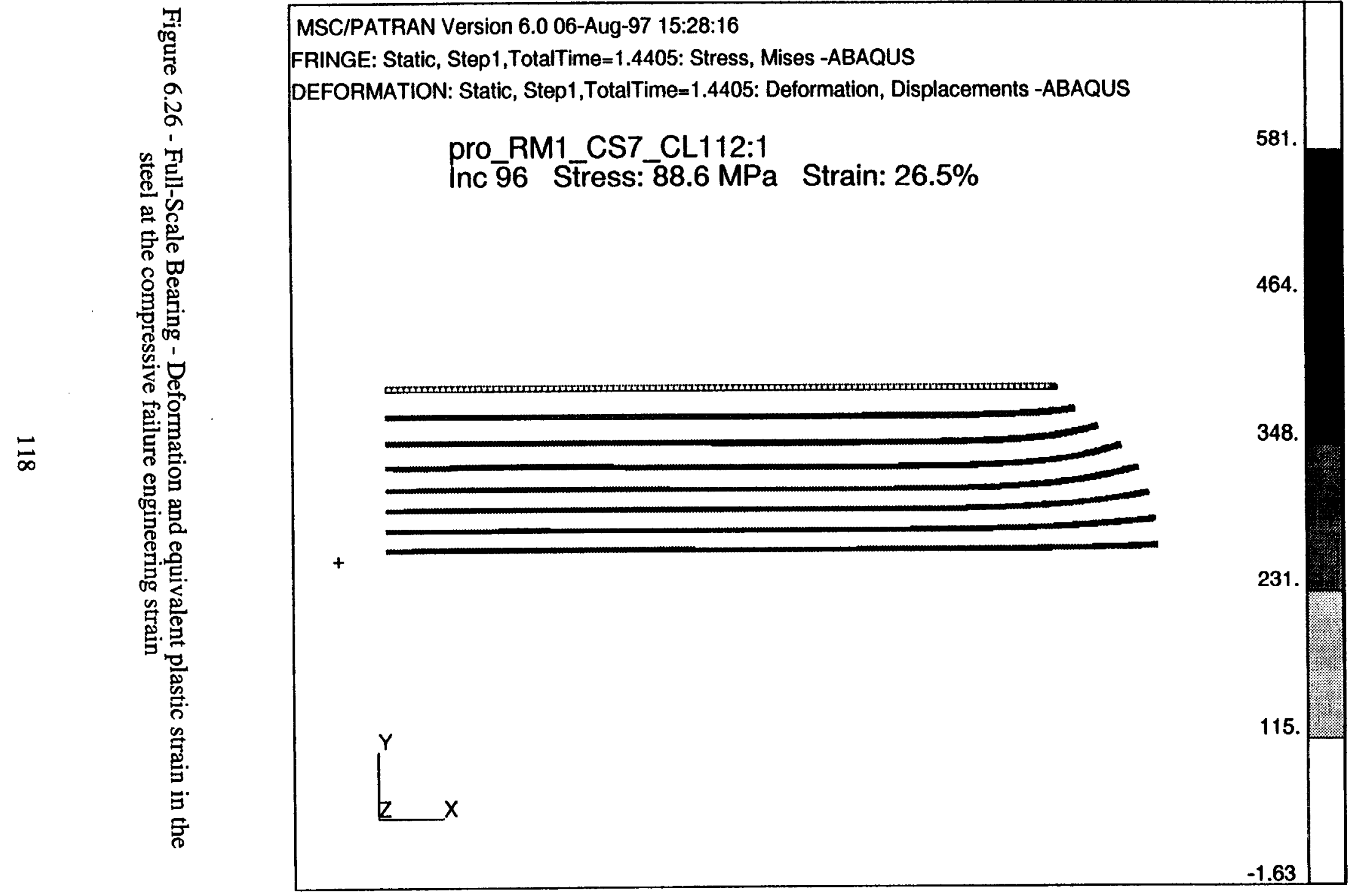




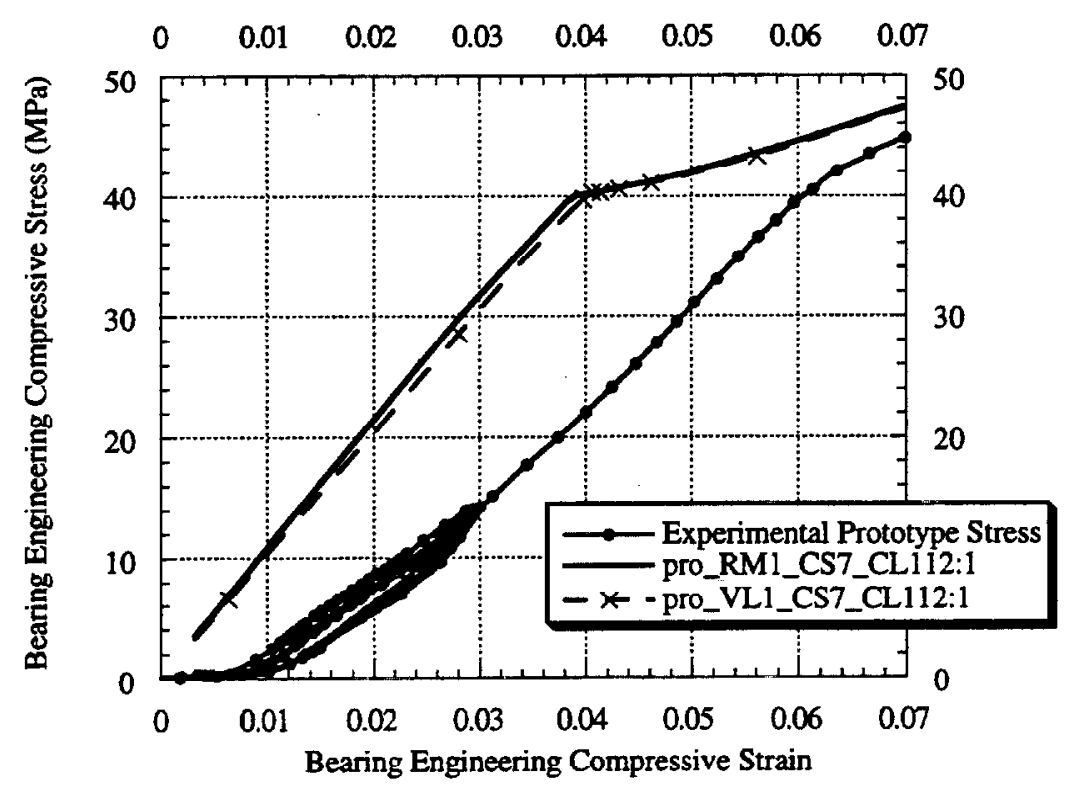

Figure 6.27 - Low strain region comparison for the prototype between the experimental engineering stress-strain curve and the ABAQUS finite element runs based on RM1 and VL

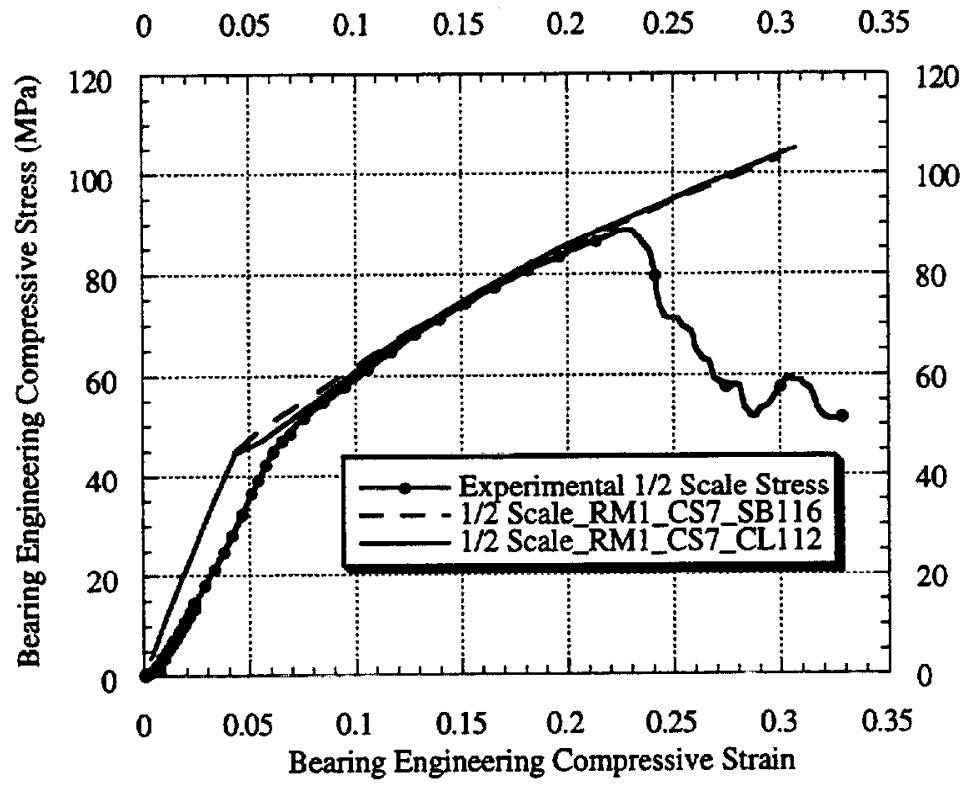

Figure 6.28 - Comparison for the 1/2-Scale Bearing model between the experimental engineering stress-strain curve and the ABAQUS finite element runs using the chemlocked steel data (CL1-12) versus the sandblasted steel data (SB1-16) 


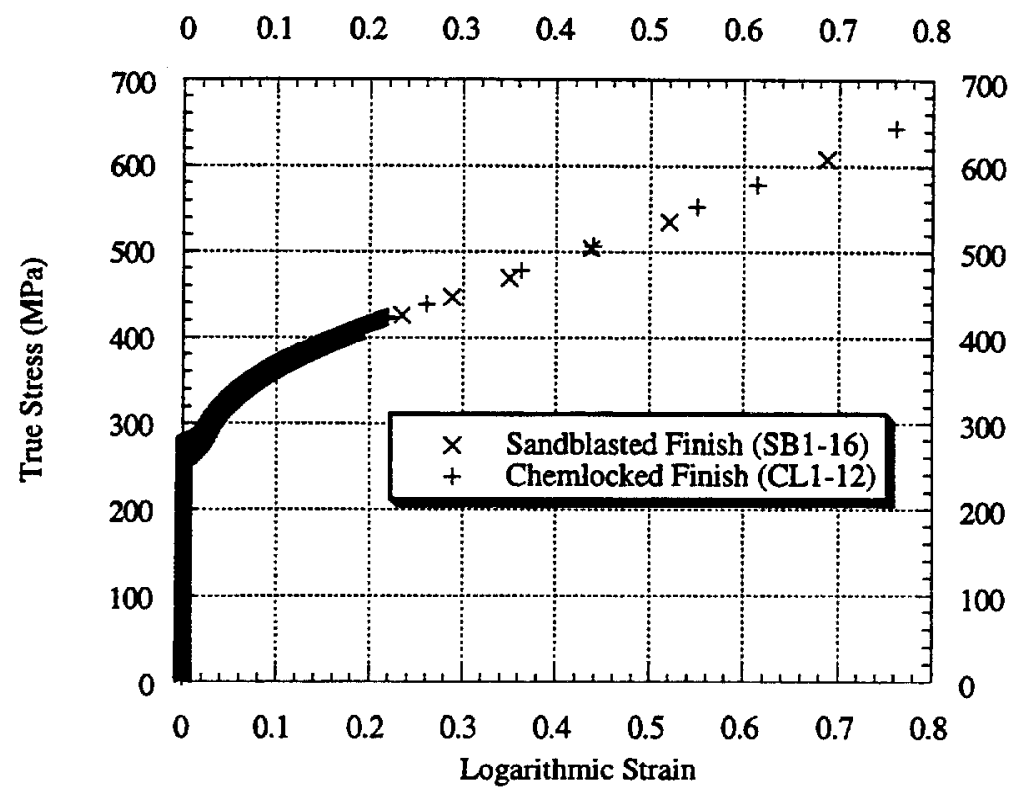

Figure 6.29 - Comparison between the true stress-strain curves of the chemlocked steel data (CL1-12) versus the sandblasted steel data (SB1-16)

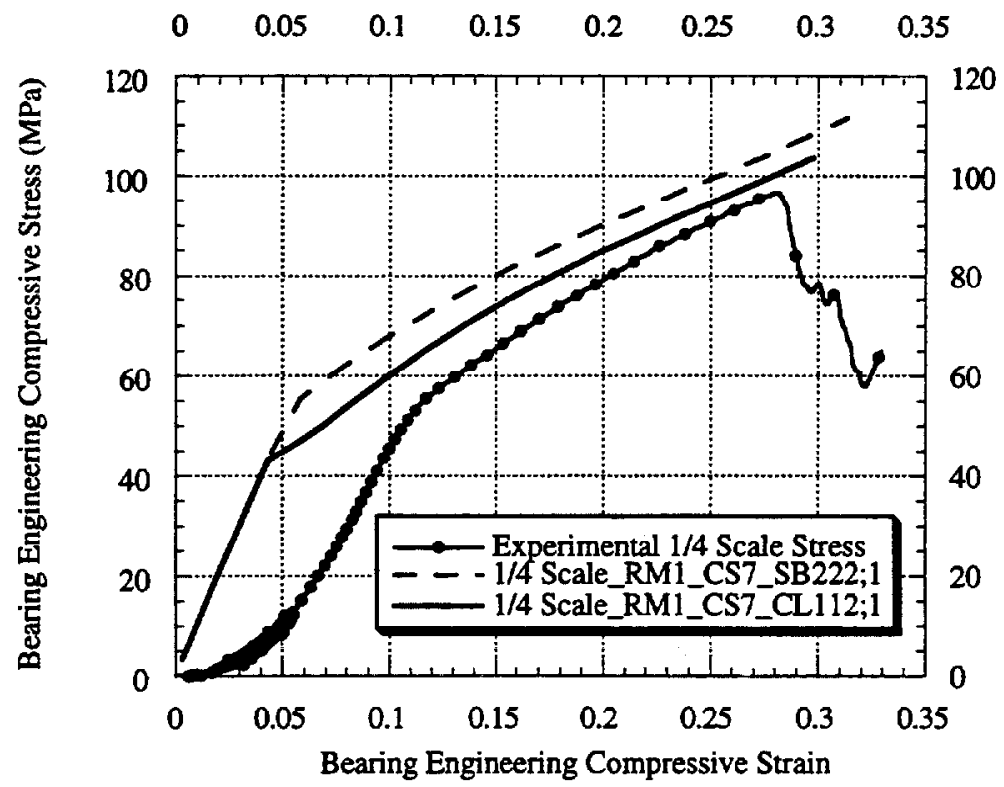

Figure 6.30 - Comparison for the 1/4-Scale Bearing model between the experimental engineering stress-strain curve and the ABAQUS finite element runs using the chemlocked steel data (CL1-12) versus the sandblasted steel data (SB2-22) 


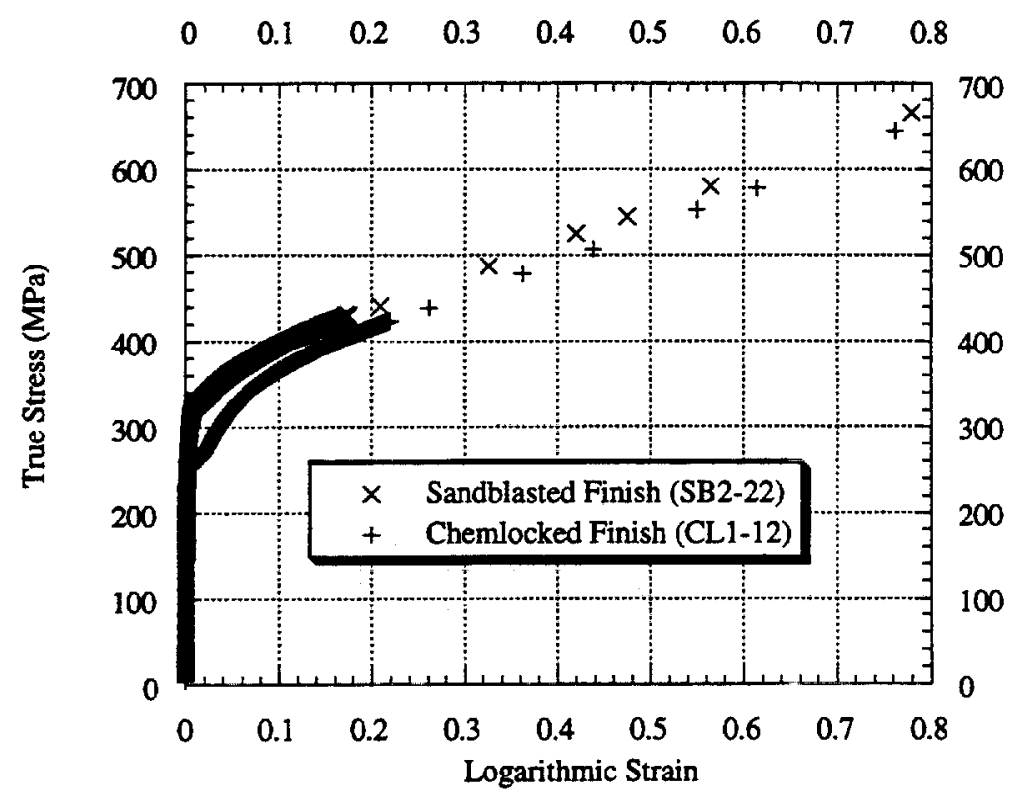

Figure 6.31 - Comparison between the true stress-strain curves of the chemlocked steel data (CL1-12) versus the sandblasted steel data (SB2-22)

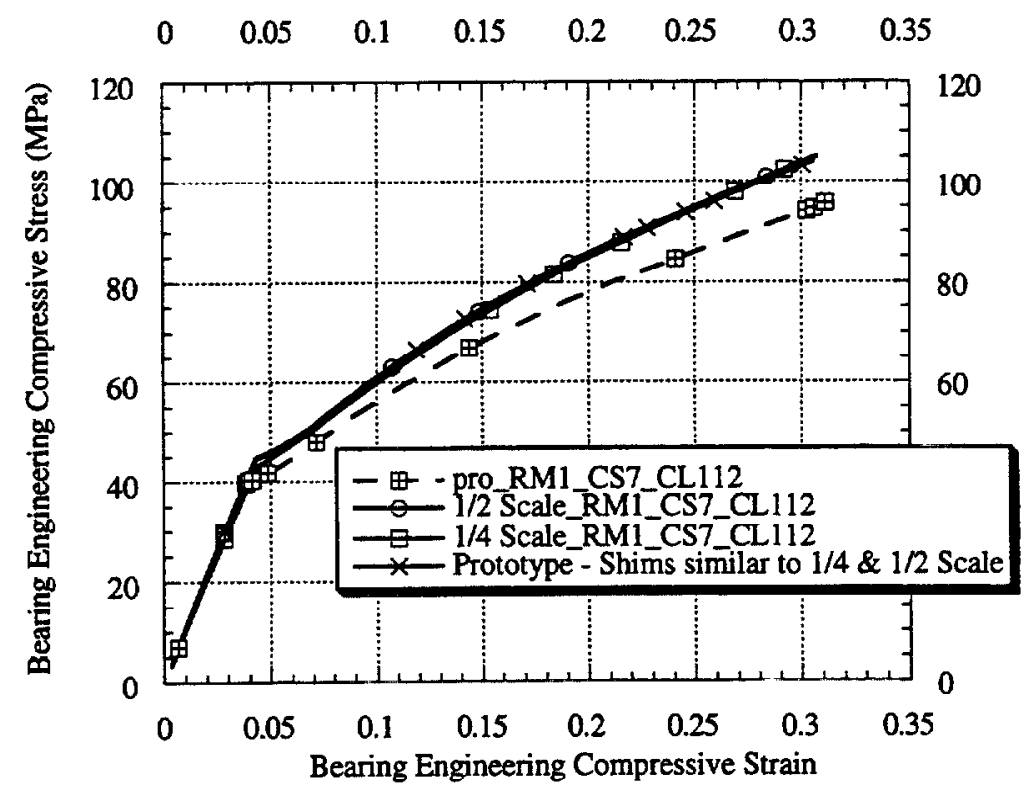

Figure 6.32 - Comparison between the ABAQUS finite element runs for the prototype, $1 / 2$ and $1 / 4$ scale models using geometrically nonsimilar and similar shims for the prototype 


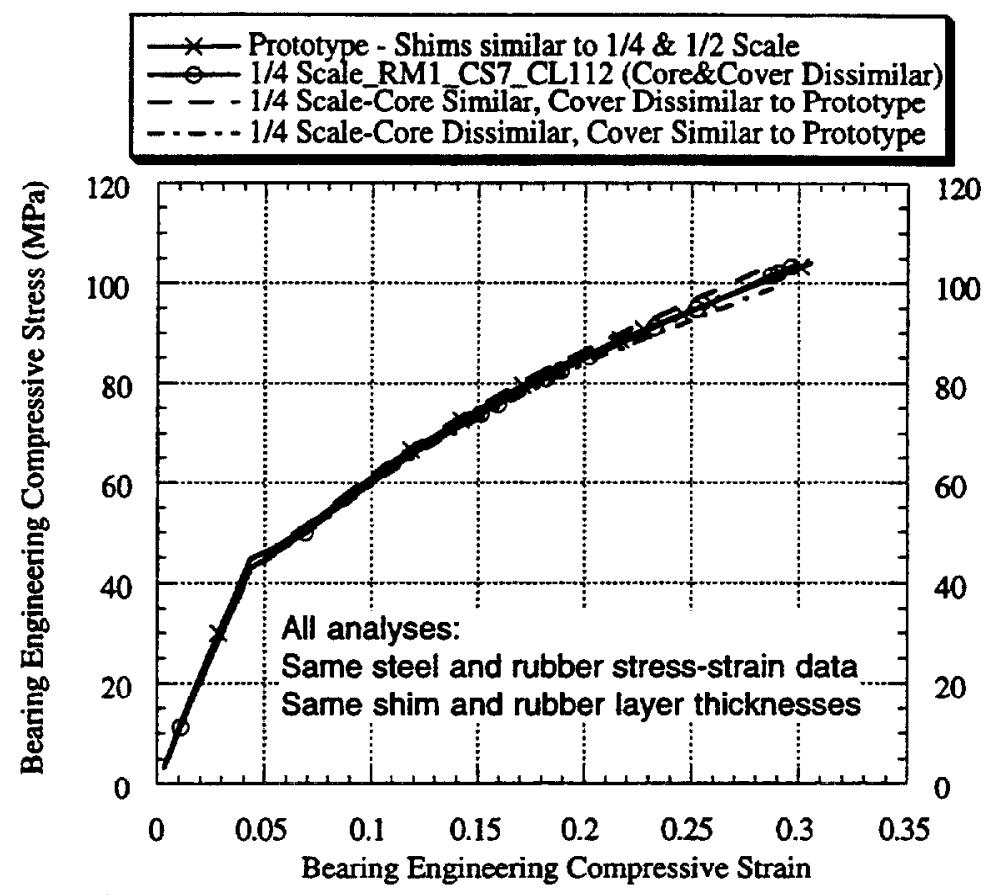

Figure 6.33 - Separate and combined effects of core and cover layer dissimilarities on the response of the 1/4-Scale Bearing 


\section{CONCLUSIONS}

Control of structural vibrations through the use of elastomeric bearings is a very promising technology. It is important to have a good understanding of the loads that may cause failure of elastomeric bearings, so that a design engineer may have an accurate assessment of the factor of safety built into his or her design. As a step in this direction, in this study we have documented failure loads of elastomeric bearings in compression, in order to determine if scale models can be used to predict the failure load of full-scale bearings. Testing of full-scale elastomeric bearings to failure in compression is not possible in most testing facilities due to the limitation of loading capacity. Even though the elastomeric bearings were designed to be exactly geometrically similar, certain dissimilarities were present due to manufacturing errors and to the use of standard manufacturing bearing molds. These dissimilarities have been analyzed using finite element models to determine their influence on the overall performance of the bearings.

The findings and conclusions of this study are summarized below:

1. In order to predict failure loads of full scale bearings from scale models, the stressstrain responses of full-scale and scale models must be nearly identical. Assuming identical rubber and steel properties among all bearings, the use of geometrically similar shims and rubber layers resulted in virtually identical stress-strain responses for all bearings, in spite of the dissimilarities in the central alignment hole and cover layer. The use of a 12 gage shim ( $2.667 \mathrm{~mm}$ thick) instead of a geometrically similar 11 gage shim (3.048 mm thick) for the Full-Scale Bearing reduced the stresses significantly $(10 \%$ reduction at a strain of $0.25 \mathrm{~m} / \mathrm{m})$. Therefore, geometric similarity in shim thickness and rubber thickness is most important. In actual practice, failure stresses may vary due to material imperfections and imperfect manufacture.

2. The effects of the dissimilarities in the scale models in the central alignment holes and the cover layers are less important than shim and rubber thickness, and in this particular case cancel each other out. The dissimilarities are most prevalent for the 1/4-Scale Bearing, and using a similar central alignment hole (everything else unchanged) for the 1/4-Scale Bearing increases the stress by approximately $3 \%$ at a strain of $0.25 \mathrm{~m} / \mathrm{m}$, and using a similar cover layer (everything else unchanged) decreases the stress by the same amount at $0.25 \mathrm{~m} / \mathrm{m}$. For the Full-Scale Bearing, the diameter of the center alignment hole was $50.1 \mathrm{~mm}$, or $6.6 \%$ of the bonded diameter. The diameter of the central alignment hole for the 1/4-Scale Bearing was $141 \%$ larger than it should have been in order to be geometrically similar to the Full-Scale Bearing, resulting in a smaller bonded area than if it were geometrically similar. The cover layer for the Full-Scale Bearing was $19 \mathrm{~mm}$ thick, or $5.0 \%$ of the bonded radius. The cover layer for the 1/4-Scale Bearing was $300 \%$ larger than it should have been in order to be geometrically similar to the Full-Scale Bearing.

3. In the experimental bearing tests, the $1 / 2$-Scale Bearing compressive stress-strain curve is stiffer than the Full-Scale's curve in the plastic range of the bearing, i.e. between initial shim yielding and bearing failure. This difference in stiffness can be attributed to the fact that the shims of the Full-Scale Bearing are less than twice the thickness of the $1 / 2$-Scale Bearing. The finite element analysis shows that bearings with geometrically similar shims and identical steel properties have 
identical compressive stress-strain responses. However, the experimental 1/4 Scale Bearing stress-strain response is stiffer than the 1/2-Scale Bearing response in the bearing's plastic region, and shows a higher stress at initial shim yielding, in spite of similarity in shim thickness between these models. Tensile tests on 22gage sandblasted coupons, which were used in the 1/4-Scale Bearing, showed a yield stress approximately $10 \%$ higher than the 16-gage sandblasted samples (used in the $1 / 2$-Scale Bearing), and stresses in the plastic range approximately $4 \%$ to $6 \%$ higher. The shims for our 1/4-Scale Bearing (22-gage) are only $0.762 \mathrm{~mm}$ thick, much thinner than normally used in bearing construction. The cold rolling process used in the manufacturing of this sheet steel and the scouring of the metal surface due to sandblasting may have modified its mechanical properties.

4. The Valanis-Landel function was used to extend the experimental rubber stressstrain data (uniaxial tension and compression) to the planar tensile (pure shear) mode. The planar tensile stress values are slightly higher than the uniaxial tensile stresses, which agrees with experimental data reported by Treloar (1943). Furthermore, equibiaxial stresses found from the conversion of uniaxial compressive data agree with the equibiaxial stresses found from the ValanisLandel function. Using only uniaxial data to determine the elastic constants, the Ogden Strain Energy Potential, which is a special case of the Valanis-Landel function, is able to predict the planar tensile stresses, while the Polynomial Strain Energy Potential with second order terms $(\mathrm{N}=2)$ overestimates the planar tensile stresses. While the qualitative agreement with Treloar's experimental data is compelling, the definitive proof of the ability of the Valanis-Landel function to accurately predict planar stresses from uniaxial stresses requires a comparison with experimental planar stress data. In the future, it would be interesting to compare experimental planar stresses to those predicted by the Valanis-Landel function from only uniaxial data.

5. The finite element results on the bearings suggest that the compressive stress-strain response of the bearings, for a given steel model, is dependent primarily on the bulk modulus of rubber, and for finite element studies where the hydrostatic rubber stress dominates, experimental determination of the bulk modulus may be all that is necessary to adequately represent the strain energy function. Stressstrain data in the uniaxial, equibiaxial, and planar deformation modes were used to find the deviatoric parameters of the strain energy function. The hydrostatic parameters were found from the volumetric test data and were used throughout the analysis when compressibility was incorporated in the model, hence no differences were observed in the hydrostatic pressure.

6. In the actual bearings, the central alignment hole is filled with rubber. Including this central core of rubber in the finite element model resulted in convergence problems, most likely due to excessive deformation of the rubber elements in the core adjacent to the shims. Excluding the core resulted in no measurable difference in the stress-strain response of the bearing. The core represents $0.4 \%$ of the bonded area (including the core) for the Full-Scale Bearing, $0.6 \%$ for the $1 / 2$ Scale Bearing, and $2.5 \%$ for the $1 / 4-$ Scale Bearing.

7. The inclusion of a compressible rubber model had little affect on the predicted failure stress, even though the initial compressive modulus was much closer to the compressive modulus in the experiments. The stress at the strain at which the Full-Scale Bearing failed was $90.3 \mathrm{MPa}$ for the finite element model based on 
rubber incompressibility, which is only $1 \%$ larger than the failure stress with the inclusion of compressibility in the finite element model $(88.9 \mathrm{MPa})$. The FullScale Bearing failed at $90 \mathrm{MPa}$, which falls between the incompressible and compressible finite element results. The initial compressive modulus of the model of the Full-Scale Bearing was $1745 \mathrm{MPa}$ when an incompressible rubber model was used, which is $70 \%$ larger than the initial compressive modulus when a compressible rubber model was used (1025 MPa). The shape factor of the FullScale Bearing rubber layers was 15 (same for scale models), and the thickness of the 12 gage shims $(2.67 \mathrm{~mm})$ was $21 \%$ of the thickness of the rubber layers $(12.7$ $\mathrm{mm}$ or $1 / 2 \mathrm{in}$.). 


\section{BLANK PAGE}




\section{REFERENCES}

ABAQUS/Standard Users Manual, 1995a, Version 5.5, HKS, Inc.

ASTM D 395-89 (Reapproved 1994), Standard Test Methods for Rubber Property Compression Set.

ASTM D 412-92, Standard Test Methods for Vulcanized Rubber and Thermoplastic Rubbers and Thermoplastic Elastomers - Tension, Test Method A.

ASTM D 429-81, Standard Test Methods for Rubber Property - Adhesion to Rigid Substrates, Method B.

ASTM D 1149-91, Standard Test Method for Rubber Deterioration - Surface Ozone Cracking in a Chamber.

ASTM D 2240-91, Standard Test Method for Rubber Property - Durometer Hardness, Durometer A.

ASTM D 3182-89 (Reapproved 1994), Standard Practice for Rubber - Materials, Equipment, and Procedures for Mixing Standard Compounds and Preparing Standard Vulcanized Sheets.

ASTM D 4014-89, Standard Specification for Plain and Steel-Laminated Elastomeric Bearings for Bridges, Annex A1, Determination of Shear Modulus.

ASTM E 8-95a, Standard Test Methods for Tension Testing of Metallic Materials.

Brown, R.P. (1986), Physical Testing of Rubber, 2nd ed., Elsevier Applied Science Publishers.

Chen, J.S., Pan, C., 1996, “A Pressure Projection Method for Nearly Incompressible Rubber Hyperelasticity, Part 1: Theory," Joumal of Applied Mechanics, Vol. 63, pp.862-868.

Dieter, George E., 1976, Mechanical Mettalurgy.

Dowling, Norman E., 1993, Mechanical Behavior of Metals - Engineering Methods for Deformation, Fracture, and Fatigue.

Eringen, A. Cemal, 1989, Mechanics of Continua.

Kearsley, E.A. and Zapas, L.J., 1980, "Some Methods of Measurement of an Elastic Strain-Energy Function of the Valanis-Landel Type," Journal of Rheology, Vol. 24, pp. 483-500.

McKenna, G.B. and Zapas, L.J., 1983, "Experiments on the small-strain behaviour of crosslinked natural rubber: 2. Extension and compression," POLYMER, Vol. 24 (4), pp. 1502-1506. 
Minor, J.C. and Egen, R.A. (1970), Elastomeric Bearing Research, National Cooperative Highway Research Program Report (NCHRPR) 109.

NISTIR 5800, 1996, "Guidelines for Pre-Qualification, Prototype and Quality Control Testing of Seismic Isolation Systems," National Institute of Standards and Technology.

Peng, S.H., and Chang, W.V. (1997), "A Compressible Approach in Finite Element Analysis of Rubber-Elastic Materials," Computers \& Structures, Vol. 62, pp. 573-593.

Roeder, C.W, Stanton, J.F., and Taylor, A.W. (1987), Performance of Elastomeric Bearings, NCHRPR 298.

Stanton, J.F. and Roeder, C.W. (1982), Elastomeric Bearings Design, NCHRPR 248.

Sussman, T.S. and Bathe, K.J., 1987, "A Finite Element Formulation for Incompressible Elastic and Inelastic Analysis," Computers \& Structures, Vol. 26, pp. 357-409.

Treloar, L.R.G. (1943), "Stress-Strain Data for Vulcanized Rubber Under Various Types of Deformation," Transactions of the Faraday Society, Vol. 40, pp. 59-70.

Treloar, L.R.G. (1975), The Physics of Rubber Elasticity, 2nd ed., Oxford University Press.

Valanis, K.C. and Landel, R.F., 1967, “The Strain Energy Function of a Hyperelastic Material in Terms of the Extension Ratios," Journal of Applied Physics, Vol. 38 , Number 7, pp. 2997-3001.

Yeoh, O.H., 1993, "Some Forms of the Strain Energy Function for Rubber," Rubber Chemistry and Technology, pp. 754-771. 


\section{APPENDIX A}

\section{SOME ELEMENTARY RESULTS IN LARGE STRAIN KINEMATICS}

The following brief description of large strain kinematics is based largely on Hjelmstad's "Fundamentals of Structural Mechanics" (1997). The detailed implementation of the kinematics and constitutive theory used for rubber and steel modeling in ABAQUS can be found in the ABAQUS/Standard Users Manual (1995a) and the ABAQUS Theory Manual (1995b).

In one dimension, the stretch $\lambda \equiv \frac{l}{l_{o}}$ is the ratio of the deformed length of a line to the original length of the line. In terms of the stretch, familiar definitions of strain are

$$
\begin{gathered}
\text { Engineering Strain }=\frac{l-l_{o}}{l_{o}}=\lambda-1 ; \\
\text { Lagrangian Strain }=\frac{1}{2}\left(\frac{l^{2}-l_{o}^{2}}{l_{o}^{2}}\right)=\frac{1}{2}\left(\lambda^{2}-1\right) ; \\
\text { Natural Strain }=\frac{l-l_{o}}{l}=1-\frac{1}{\lambda} ; \\
\text { Eulerian Strain }=\frac{1}{2}\left(\frac{l^{2}-l_{o}^{2}}{l^{2}}\right)=\frac{1}{2}\left(1-\frac{1}{\lambda^{2}}\right), \text { and } \\
\ln \text { Strain }==\int_{l_{0}}^{l} \frac{d l}{l}=\ln \left(\frac{l}{l_{0}}\right)=\ln \lambda .
\end{gathered}
$$

More generally, the stretch can vary from point to point in a one-dimensional rod, so that a rod of initial length $l_{0}$, when deformed, has a length $l=\int_{0}^{l_{0}} \lambda(x) d x$. If the strain in the rod is homogeneous, i.e. the same at each point, then

$$
l=\lambda \int_{0}^{l_{0}} d x \Rightarrow \lambda=\frac{l}{l_{0}} \text {. }
$$

To get a general formulation for the stretch at a point in three dimensions, consider Figure A.1. Curve $C$ is any curve in the undeformed body that passes through point $P$, and is deformed into the line $\Phi(C)$. If we parametrize $C$ by s, the $\lim _{\Delta s \rightarrow 0}\left(\frac{\underline{X}(s+\Delta s)-\underline{X}(s)}{\Delta s}\right)=\frac{d \underline{X}}{d s}$, and $\frac{d \underline{X}}{d s}$ is a unit vector at $\mathrm{P}$ that is tangent to $\mathrm{C}$ at $\mathrm{P}$, because the length of the vector $\underline{X}(s+\Delta s)-\underline{X}(s)$ approaches the arclength $\mathrm{s}$ as $\Delta s$ approaches zero. The $\lim _{\Delta s \rightarrow 0}\left(\frac{\underline{x}(s+\Delta s)}{\Delta s}\right)=\frac{d \underline{x}}{d s}$ is a vector tangent to $\Phi(\mathrm{C})$ at $\Phi(\mathrm{P})$, but 
is not a unit vector. The stretch at point $P$ in the direction given by parameter $s$ along curve $C$ is $\lambda=\frac{d \underline{x}}{d s}$.
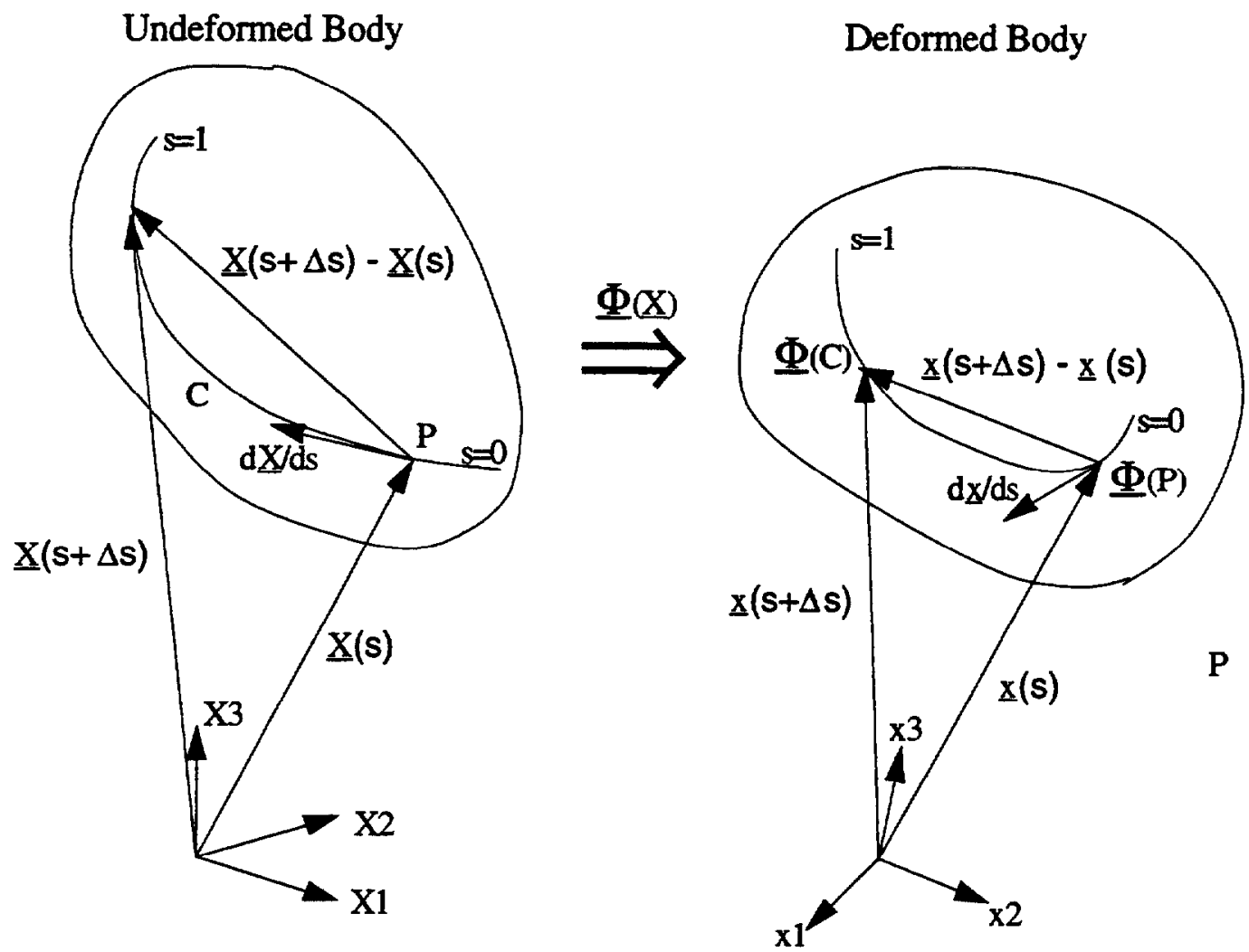

Figure A.1 - Mapping from the Undeformed to Deformed Configuration

There are an infinite number of lines that pass through the point $P$, each defining a direction with an associated stretch, so the state of deformation at $\mathrm{P}$ can be thought of as a infinite collection of stretches and their associated directions. To get a general description which captures all of the information, we can relate the vector $\mathrm{d} \underline{\mathrm{x}} / \mathrm{ds}$ to $\mathrm{d} \underline{\mathrm{X}} / \mathrm{ds}$. Writing $\underline{\mathrm{x}}(\mathrm{s})$ in terms of the deformed body's coordinate system, we get $\underline{x}(s)=x_{1}(s) \underline{i}_{1}+x_{2}(s) \underline{i}_{2}+x_{3}(s) \underline{i}_{3}$. In terms of the mapping function $\mathrm{E}$, this can be expressed as

$$
\underline{x}(s)=\Phi_{1}(\underline{X}(s)) \underline{i}_{1}+\Phi_{2}(\underline{X}(s)) \underline{i}_{2}+\Phi_{3}(\underline{X}(s)) \underline{i}_{3}
$$

Taking the derivative of Equation (A1.6) with respect to $s$, we get 


$$
\begin{aligned}
\frac{d \underline{x}(s)}{d s}= & \left(\frac{\partial \Phi_{1}(\underline{X}(s))}{\partial X_{1}(s)} \frac{d X_{1}(s)}{d s}+\frac{\partial \Phi_{1}(\underline{X}(s))}{\partial X_{2}(s)} \frac{d X_{2}(s)}{d s}+\frac{\partial \Phi_{1}(\underline{X}(s))}{\partial X_{3}(s)} \frac{d X_{3}(s)}{d s}\right) \underline{i}_{1} \\
& +\left(\frac{\partial \Phi_{2}(\underline{X}(s))}{\partial X_{1}(s)} \frac{d X_{1}(s)}{d s}+\frac{\partial \Phi_{2}(\underline{X}(s))}{\partial X_{2}(s)} \frac{d X_{2}(s)}{d s}+\frac{\partial \Phi_{2}(\underline{X}(s))}{\partial X_{3}(s)} \frac{d X_{3}(s)}{d s}\right) \underline{i}_{2} \\
& +\left(\frac{\partial \Phi_{3}(\underline{X}(s))}{\partial X_{1}(s)} \frac{d X_{1}(s)}{d s}+\frac{\partial \Phi_{3}(\underline{X}(s))}{\partial X_{2}(s)} \frac{d X_{2}(s)}{d s}+\frac{\partial \Phi_{3}(\underline{X}(s))}{\partial X_{3}(s)} \frac{d X_{3}(s)}{d s}\right) \underline{i}_{3}
\end{aligned}
$$

Dropping the reference to the arclength parameter s, and putting Equation (A1.7) in matrix form, we get

$$
\begin{aligned}
& \left\{\begin{array}{l}
d x_{1} \\
d x_{2} \\
d x_{3}
\end{array}\right\}=\left(\begin{array}{lll}
\frac{\partial \Phi_{1}}{\partial X_{1}} & \frac{\partial \Phi_{1}}{\partial X_{2}} & \frac{\partial \Phi_{1}}{\partial X_{3}} \\
\frac{\partial \Phi_{2}}{\partial X_{1}} & \frac{\partial \Phi_{2}}{\partial X_{2}} & \frac{\partial \Phi_{2}}{\partial X_{3}} \\
\frac{\partial \Phi_{3}}{\partial X_{1}} & \frac{\partial \Phi_{3}}{\partial X_{2}} & \frac{\partial \Phi_{3}}{\partial X_{3}}
\end{array}\right)\left\{\begin{array}{l}
d X_{1} \\
d X_{2} \\
d X_{3}
\end{array}\right\} \text {, or } \\
& d \underline{x}=\underline{\underline{F}} d \underline{X}
\end{aligned}
$$

$\underline{\underline{F}}$ is called the "deformation gradient," and is a tensor of rank two. By the polar decomposition theorem, $\underline{\underline{F}}=\underline{\underline{R}} \underline{\underline{U}}$, where $\underline{\underline{U}}$ changes the length of a vector without changing its direction, and $\underline{\underline{R}}$ rotates the vector without changing its length. The Right Cauchy-Green Deformation Tensor is defined as $\underline{\underline{C}} \equiv \underline{\underline{F}}^{T} \underline{\underline{F}}$, which is by definition symmetric. $\underline{\underline{C}}$ contains information on deformation only, as can be shown by $\underline{\underline{C}} \equiv \underline{\underline{F}}^{T} \underline{\underline{F}}=(\underline{\underline{R}} \underline{\underline{U}})^{T} \underline{\underline{R}} \underline{\underline{U}}=\underline{\underline{U}}^{T} \underline{\underline{R}}^{T} \underline{\underline{R}} \underline{\underline{U}}=\underline{\underline{U}}^{T} \underline{\underline{U}}$. Since $\mathrm{R}$ is a rotation only, $\underline{\underline{\underline{I}}}=\underline{\underline{R}}^{T} \underline{\underline{R}}$. The Lagrangian Strain in three dimensions is $\underline{\underline{E}}=\frac{1}{2}(\underline{\underline{C}}-\underline{\underline{I}})$. Since $\underline{\underline{C}}$ is symmetric, $\underline{\underline{E}}$ is also symmetric. In indicial notation, $E_{i j}=u_{i, j}+u_{j, i}+u_{k, i} u_{k, j}$.

$$
\begin{gathered}
\text { In terms of principal stretches, } \underline{\underline{C}}=\left(\begin{array}{ccc}
\lambda_{1}^{2} & 0 & 0 \\
0 & \lambda_{2}^{2} & 0 \\
0 & 0 & \lambda_{3}^{2}
\end{array}\right) \text {. The invariants of } C \text { are } \\
I_{1}=\lambda_{1}^{2}+\lambda_{2}^{2}+\lambda_{3}^{2} \\
I_{2}=\lambda_{1}^{2} \lambda_{2}^{2}+\lambda_{1}^{2} \lambda_{3}^{2}+\lambda_{2}^{2} \lambda_{3}^{2} \\
I_{3}=\lambda_{1}^{2} \lambda_{2}^{2} \lambda_{3}^{2}
\end{gathered}
$$


The ratio of the current volume to the original volume is $J=\sqrt{I_{3}}=\operatorname{det}(\underline{\underline{F}})=\lambda_{1} \lambda_{2} \lambda_{3}$. As noted in Section 3.2, in the mixed formulation revised invariants are used to remove the volume change. In ABAQUS, the revised invariants, written in terms of the deviatoric stretches, $\bar{\lambda}_{i}=J^{-1 / 3} \lambda_{i}$, are

$$
\begin{gathered}
\bar{I}_{1}=\bar{\lambda}_{1}^{2}+\bar{\lambda}_{2}^{2}+\bar{\lambda}_{3}^{2} \\
\bar{I}_{2}=\bar{\lambda}_{1}^{2} \bar{\lambda}_{2}^{2}+\bar{\lambda}_{1}^{2} \bar{\lambda}_{3}^{2}+\bar{\lambda}_{2}^{2} \bar{\lambda}_{3}^{2}
\end{gathered}
$$

As shown in Section 3.2, the constitutive models for rubber are written in terms of the strain invariants or principal stretches. ABAQUS provides output in terms of strain. For steel, the strain is decomposed into an elastic and inelastic portion. 


\section{APPENDIX B \\ PROGRAM LISTING FOR DETERMINATION OF THE DERIVATIVE OF THE VALANIS-LANDEL FUNCTION}

$1^{*}$ This program uses the Kearsley and Zappas separation algorithm to calculate the Valanis-Landel function from uniaxial compression $*$ / and tension data.

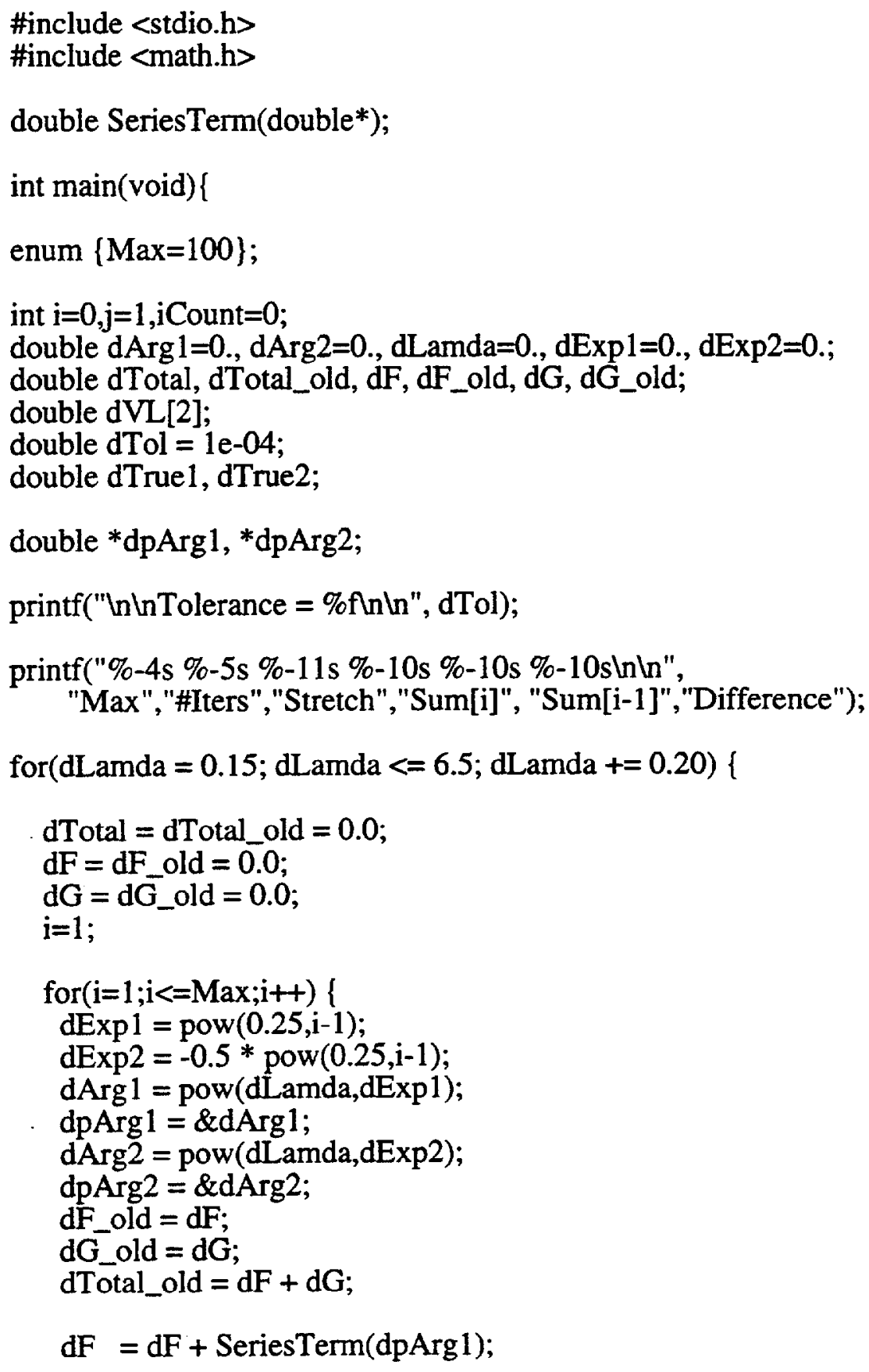




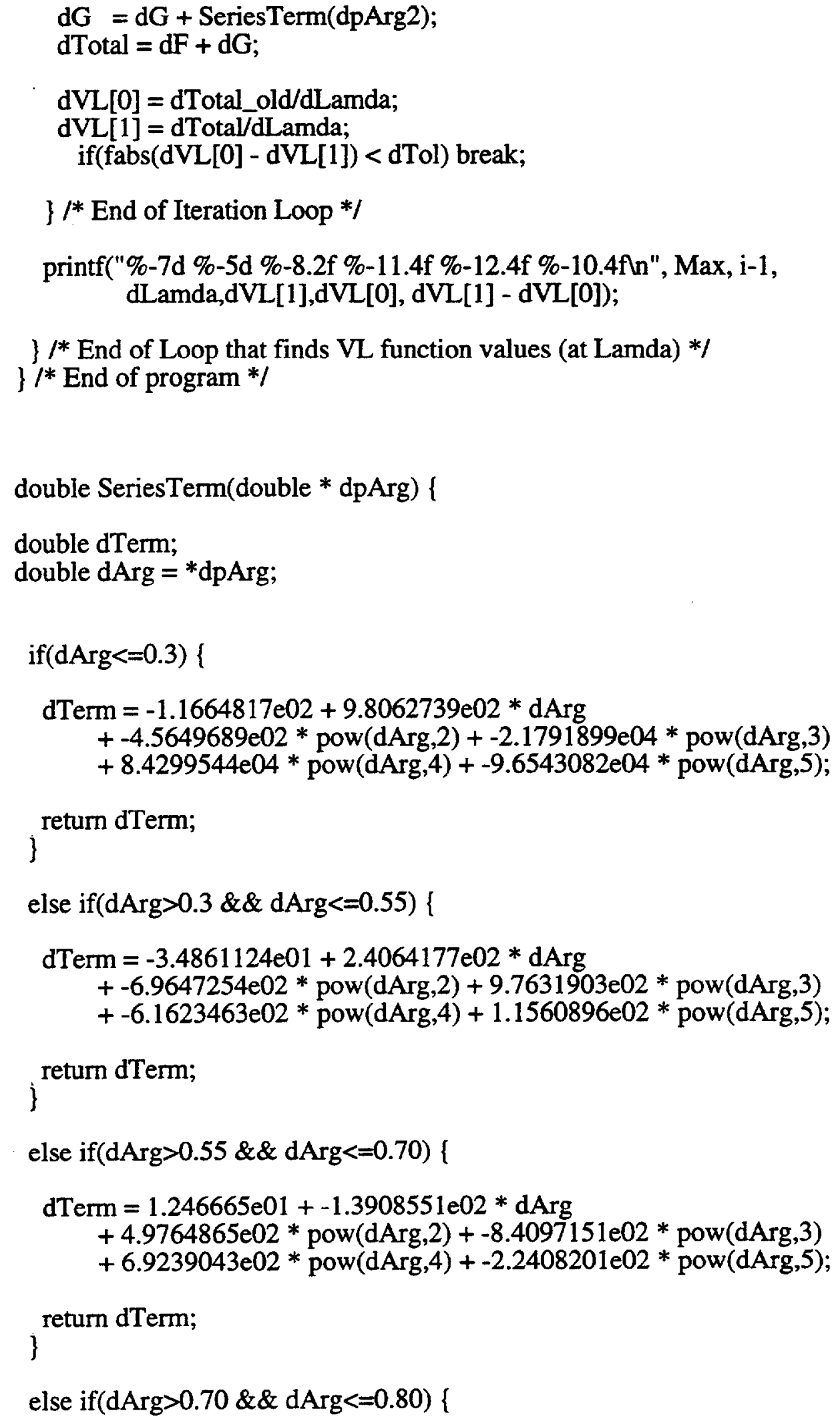




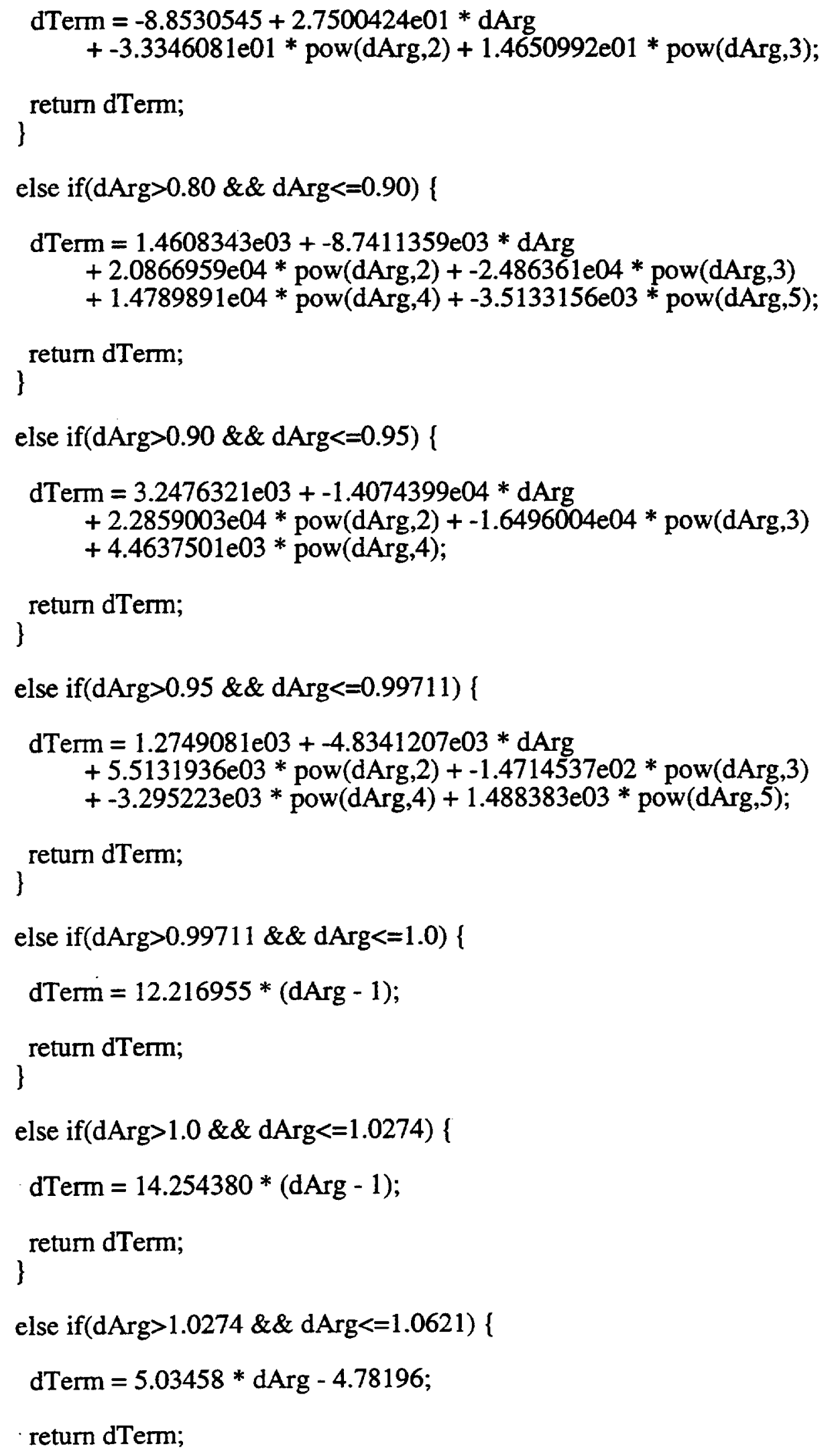


else if (dArg $>1.0621 \& \&$ dArg $<=2.4971)$ (

$\mathrm{dTerm}=-1.9199173 \mathrm{e} 03+9.8937267 \mathrm{e} 03 * \mathrm{dArg}$ $+-2.2356421 \mathrm{e} 04 *$ pow $(\mathrm{dArg}, 2)+2.9064259 \mathrm{e} 04 *$ pow(dArg,3)

$+-2.3956019 \mathrm{e} 04 *$ pow $(\mathrm{dArg}, 4)+1.2989462 \mathrm{e} 04 *$ pow $(\mathrm{dArg}, 5)$

$+-4.6372447 \mathrm{e} 03 *$ pow $(\mathrm{dArg}, 6)+1.0523308 \mathrm{e} 03 *$ pow(dArg, 7$)$

$+-1.3795277 \mathrm{e} 02 *$ pow $(\mathrm{dArg}, 8)+7.9740265 *$ pow(dArg,9);

return dTerm;

)

else \{

$\mathrm{dTerm}=-1.8322249 \mathrm{e} 03+4.8664372 \mathrm{e} 03 * \mathrm{dArg}$

$+-5.5502795 \mathrm{e} 03 *$ pow $(\mathrm{dArg}, 2)+3.5819692 \mathrm{e} 03 *$ pow $(\mathrm{dArg}, 3)$

$+-1.4448741 \mathrm{e} 03 *$ pow $(\mathrm{dArg}, 4)+3.7870137 \mathrm{e} 02 *$ pow $(\mathrm{dArg}, 5)$

$+-6.4597472 \mathrm{e} 01 *$ pow $(\mathrm{dArg}, 6)+6.9249393 *$ pow $(\mathrm{dArg}, 7)$

$+-4.2390496 \mathrm{e}-01 *$ pow $(\mathrm{dArg}, 8)+1.1303178 \mathrm{e}-02 *$ pow $(\mathrm{dArg}, 9)$;

return dTerm;

\} 